\title{
Presented Abstracts from the Thirty Third Annual Education Conference of the National Society of Genetic Counselors (New Orleans, LA, September 2014)
}

\author{
Emily Edelman ${ }^{1 *}$, Beverly M. Yashar ${ }^{2}$, Katie Darin $^{3}$ \\ ${ }^{1}$ The Jackson Laboratory, 600 Main Street, Bar Harbor, ME 04609, USA $;{ }^{2}$ Department of Human Genetics, The University of Michigan, Ann Arbor, MI, \\ USA ${ }^{3}$ National Society of Genetic Counselors Executive Office, Chicago, IL, USA
}

\section{Awards Papers}

The Beth Fine Kaplan Student Abstract Award

Genetic Counselors as Choice Architects: Some Considerations for Presenting Genetic Testing Decisions in a Complex Choice Environment

M. Barr' ${ }^{1}$, B. Biesecker ${ }^{1}$, D. Roter $^{2}$

1. Genetic Counseling Training Program, Johns Hopkins University/National Human Genome Research Institute

2. Johns Hopkins University Bloomberg School of Public Health

As genetic technologies have evolved, genetic testing options have proliferated which has created new challenges in the field of genetic counseling, including how to best present genetic testing choices. In some cases, client decisions about genetic testing that formerly involved deciding whether or not to undergo testing, may involve choosing from multiple tests. For example, clients may need to decide among cancer panel tests, choose between noninvasive and invasive prenatal tests, and specify preferences about the return of incidental findings from whole exome sequencing tests. With more options available, decision making has become more complex and there are more ways to structure and describe choices. Additionally, research in the field of judgment and decision making has shown that subtle changes in the structure of a choice problem can have a profound impact on the choice made. As the individuals who design and present genetic testing decisions, genetic counselors have a key role as choice architects and unfortunately there is no neutral way to design a choice problem. Given this, we have a responsibility to use theory to design choices in a way that facilitates optimal medical and psychological outcomes. The objective of this presentation is to propose ways to structure genetic testing decisions by suggesting a framework for a genetic testing choice task. The framework was developed using constructs and relationships from the multidisciplinary decision making literature on how changes in the organization and description of a choice problem can influence decisions. For example, it will introduce how altering default options, the number of options, partitioning of attributes, and salience of attributes can change decision making outcomes and influence decisions. We suggest areas of future research and techniques such as discrete choice experiments to help determine which attributes of genetic testing drive decisions to build the evidence base needed to develop practice guidelines on how to present choice.

Full Member Abstract Award

Analysis of Billing and Reimbursement of Genetic Counseling Services in a Single Institution in a State Requiring Licensure

J. Leonhard ${ }^{1}$, P. Munson' ${ }^{1}$, J. Flanagan ${ }^{1}$, K. De Berg ${ }^{1}$, P. Thompson ${ }^{2}$, Q. Stein ${ }^{1}$

1. Sanford Health

2. Sanford Research

Complications with reimbursement have limited the ability to integrate trained genetic service providers into the health care system. The specific aim of this study was to assess billing and reimbursement for genetic counseling services at a single institution in a state requiring licensure (Sanford Health, Sioux Falls, SD). Patient encounters utilizing the 96040 CPT ${ }^{\circledR}$ code from $7 / 31 / 2009$ through $7 / 31 / 2013$ were reviewed. This timeframe was selected based on the requirement for genetic counselors to be licensed in SD starting in July 2009. While the purpose of licensure is to protect the public, it's possible that holding a license may secondarily increase opportunities for reimbursement if an insurance company is willing to credential a licensed provider. Exclusion criteria included billing records of patients who were seen by a physician the same day of their appointment, self-pay, Medicaid, and Medicare patients. Of the 8,630 encounters with a genetic counselor, 582 encounters were eligible for review with $52.8 \%$ receiving some level of reimbursement at an average reimbursement rate of $42.4 \%$. Two or more units of the 96040 code were billed for 123 encounters. Of those which received reimbursement for the first unit, $60.0 \%$ received some level of reimbursement for the remaining units, and $25.0 \%$ received full reimbursement for all units. A total of 31 insurance companies were billed with all but four providing some level of reimbursement. Statistical analysis confirmed 
significant differences in reimbursement rates between insurance companies that do and do not credential genetic counselors $(p<.0001)$. With regard to ICD-9 codes, 85 different codes were utilized (including V codes) with $75.3 \%$ receiving some level of reimbursement. Statistical analysis determined there is not a significant difference in reimbursement rates when a primary diagnostic ICD-9 code is used versus that of an ICD-9 V code $(p=3,585)$. Data regarding CPT ${ }^{\circledR} 96040$ has scarcely been reported. Our results and analysis will provide a useful baseline for national comparisons.

\title{
II. Platform Presentations
}

\section{Access and Service Delivery}

\section{A Comparison of Telephone and in Person Genetic Counseling from the Genetic Counselor's Perspective}

\author{
K. Burgess ${ }^{1}$, E. Carmany ${ }^{1}$, A. Trepanier ${ }^{1}$
}

\section{Wayne State University}

Growing demand for and limited geographic access to genetic counseling services is increasing the need for alternative service delivery models (SDM) like telephone genetic counseling (TGC). Thus far, there has been little research on the perspectives of genetic counselors about TGC. We created a novel online survey based on a subset of tasks from the American Board of Genetic Counseling Practice Analysis to identify whether there are differences between the tasks performed in TGC versus in person genetic counseling (IPGC). The survey used both Likert-like and open ended questions to identify differences and additional skills needed to effectively perform TGC. In total, 113 genetic counselors with experience in TGC, as defined by the NSGC SDM Task Force, completed at least part of the survey. Telephone genetic counselors most commonly practiced in commercial diagnostic laboratories $(30.1 \% ; 19 / 63)$ or university medical centers $(27.0 \% ; 17 / 63)$ providing reproductive $(46.2 \% ; 30 / 65)$, cancer $(43.2 \% ; 28 / 65)$ and/or carrier screening services $(35.5 \% ; 23 / 65)$. Using a cut-off where the 'different' category was greater than or equal to $25 \%$, respondents reported that 13 (14.8\%) of the 88 tasks studied were different for TGC. The tasks respondents indicated were most different included: "establishing rapport through verbal and nonverbal interactions" (60.2\%; 50/83), "recognizing factors affecting the counseling interaction" (47.8\%; 32/67), "assessing client/family emotions, support, etc." $(40.1 \% ; 27 / 66)$ and "educating clients about basic genetic concepts" $(35.6 \% ; 26 / 73)$. There was also a significant difference between the mean length of IPGC sessions (47.2 min) and TGC sessions (37.9 min) [ $p<.001]$. A majority $(53.8 \% ; 35 / 65)$ felt additional training in TGC was needed specifically in communicating information without visual aids and more effectively performing psychosocial assessments. In summary, a majority of genetic counseling tasks are performed similarly between TGC and IPGC. However, counselors recognize that specific training in the TGC model may be needed to address the key differences.

Perinatal Palliative Care and Bereavement: Establishing a Compassionate, Multidisciplinary Program for Families Facing a Life-Limiting Prenatal Diagnosis

\author{
S. Chadwick ${ }^{1}$, S. Kasperski ${ }^{1}$, E. Shaughnessy ${ }^{1}$, D. Munson ${ }^{1}$, J. Moldenhauer ${ }^{1}$, N. Khalek $^{1}$, J. Cole ${ }^{1}$

\section{Children's Hospital of Philadelphia}

It is estimated that 2-4\% of all neonates are born with a congenital malformation. For patients carrying fetuses with life-limiting congenital malformations or genetic conditions, the decision regarding whether to continue or end a pregnancy is challenging for not only the mother, but the entire family. Perinatal palliative care is an emerging specialty that focuses on the needs of such families who decide to a carry a pregnancy to term knowing postnatal life will be limited. We discuss how a large prenatal diagnostic center has been able to build a multidisciplinary, comprehensive programmatic approach to palliative care. Incorporating multiple subspecialties including maternal fetal medicine, nursing, neonatology, psychology, child life, social work, genetic counseling, chaplain services, and bereavement coordination, the Perinatal Palliative Care and Bereavement Program at The Children's Hospital of Philadelphia serves to provide support for the entire family from the time of initial prenatal diagnosis, through the antepartum and intrapartum periods, to the time of the fetal/neonatal loss, and well into the postpartum period. Since May 2012, 35 families from 8 states have undergone palliative care consults for a variety of indications - we will share their stories via case examples and highlight the role of genetic counselors in their care throughout the presentation. The perinatal palliative care experience allows families to actively participate with their medical team in designing a unique care plan and creating memories to honor their child. The birth plans drafted during pregnancy are shared with the patient's medical and nursing teams so that during and following delivery, the family's wishes are supported. Finally, our program provides long-term bereavement follow-up to ensure that families receive the support necessary to effectively transition back home.

\section{Show Me the Money: Half a Million Downstream Billing Generated from Three Genetic Counselors' Visits over Nine Months}

\author{
M. Dudek ${ }^{1}$, S. Lovett ${ }^{2}$, J. Nichols ${ }^{2}$ \\ 1. Vanderbilt School of Medicine \\ 2. Vanderbilt University Medical Center
}

Objective: Calculate the amount of downstream revenue generated by unique referrals to prenatal genetic counselors August 2013 to April 2014 according to clinic location and indication to an academic medical center. Background: An increasing number of genetic counselors bill for their services 
and are reimbursed. Recent studies have shown that genetic counselors save institutions money. This study provides data that downstream revenue can also be used to illustrate economic value to an institution from genetic counselors. Methods: REDCap, a web-based survey tool, was used to prospectively collect genetic counselors' recommendations. Data tracked included referral source, indication, clinic site, testing, procedures, transfers of prenatal care, and referrals to consultants. Downstream billing was captured retrospectively through EPIC billing system. Revenue was tracked ONLY for patients that without referral to the genetic counselor would otherwise not have been seen at the institution. Results: From August 2013 to April 2014, $12 \%(80 / 681)$ of the genetic counselors' patients were unique and would not have otherwise been seen at the institution. These referrals generated $\$ 453,299$ of downstream billing for an average of $\$ 5,666$ per patient. Consults for fetal anomalies followed by advanced maternal age generated the most billable services. The patients seen at the main location and the multidisciplinary fetal center were more profitable than the outreach locations. Eight percent of the 80 encounters resulted in transfers of care, $38 \%$ resulted in procedures and $31 \%$ resulted in consults. Conclusions: Tracking downstream revenue from three prenatal genetic counselor visits found $\$ 5 \mathrm{~K}$ per visit and total of almost half a million billable services. In addition to illustrating cost savings and billable face-to-face time, downstream revenue demonstrates the dollars genetic counselors contribute to an institution's bottom line.

Family History as a Predictor of Mutation Positive Status in Hypertrophic Cardiomyopathy: Refining a Genotype Risk Algorithm

\title{
L. Hipp ${ }^{1}$, S. Day ${ }^{1}$, P. Arscott ${ }^{1}$, B. Yashar ${ }^{1}$
}

\section{University of Michigan}

Introduction: Hypertrophic Cardiomyopathy (HCM) genetic testing is currently limited by high cost and low yield. To improve cost effectiveness, Gruner et al. (2013) developed a risk algorithm using patient clinical presentation to predict mutation identification. The identification of family history as a significant predictor suggests a role for pedigree analysis in improving model predictability. This study incorporates an expanded family history score into development of a risk algorithm to guide clinician decisions to pursue genetic testing for HCM diagnoses. Hypothesis: Inclusion of family history as a continuous variable in risk algorithm development increases model validity and predictive ability. Methods: Participants with HCM and previous genetic testing were selected from the University of Michigan HCM Patient Registry $(N=261)$. Participant pedigrees were scored by summing the 1 st and 2nd degree relatives diagnosed with HCM or with sudden cardiac death $<=$ age 45 . Clinical variables were tested for associations with positive mutation status by $t$-test or Pearson chi square test, with submission of significant predictors to logistic regression using mutation status as the outcome variable. Regression estimates were used to compute participant risk scores and associated predicted probabilities for mutation identification. Model validity was assessed by Pearson correlation and ROC analysis. Results: Family history score was identified as a significant predictor in both bivariate and multivariate analyses $(p=0.000)$. Inclusion of the expanded family history score improved model predictability as indicated by lower Akaike's and Bayesian Information Criterion. Probabilities predicted by the model were highly correlated with observed positive genotypes in the sample (R2=0.582, $p=0.000$ ). ROC area showed strong model discrimination ( $\mathrm{AUC}=0.837, p=0.000$ ). Conclusion: Algorithm validity is increased by incorporation of expanded family history information, highlighting the added value of detailed family history in HCM risk assessment, test referrals and cardiology care.

\section{Resource Stewardship: The Children's Hospital Colorado Laboratory Experience to Improve the Value of Expensive Genetic Reference} Laboratory Tests with Active Utilization Management

\author{
M. Lovell ${ }^{1}$, G. Pino ${ }^{1}$, A. Smith ${ }^{1}$, R. Carpenter ${ }^{1}$, W. Smith $^{1}$, C. McCloud ${ }^{1}$, W. Schwent ${ }^{1}$, R. Cole ${ }^{1}$, M. Saenz ${ }^{1}$, A. Collins ${ }^{1}$, R. Quinones ${ }^{1}$ \\ 1. Children's Hospital Colorado
}

Children's Hospital Colorado (CHCO) has undertaken a resource stewardship initiative aimed at providing appropriate care to patients with improved outcomes, and at a reduced cost; in essence increasing the value of healthcare for patients and the families who care for them. This initiative is hospital wide and includes all departments. From 2007 to 2010, send-out test expenses at the CHCO Laboratory increased annually at a rate of $33 \%$. Recognizing this as a resource stewardship opportunity, CHCO Lab embarked in 2011 on 4-pronged collaboration with all stakeholders to reduce these expenses; namely, by consolidating reference laboratories, converting miscellaneous tests to named tests, developing more in-house testing options, and focusing the utilization of send-out tests. In the fall of 2013, a test utilization management pilot project was implemented to determine if directed assistance to physicians would reduce costs without compromising patient care. Pilot tests were chosen on the basis of cost and also the frequency of orders. The options for the order, following a review by a genetic counselor were to proceed with the test as ordered, to modify the test or test strategy to a more appropriate one, or to cancel the test entirely. The pilot ran from October 7, 2013 to the end of December 2013. Overall, 123 tests were reviewed, $18 \%$ of these were either modified or cancelled. The cost savings per test was $\$ 113$. Expansion of the pilot to all tests costing over $\$ 1,000$ began April $28,2014$. Additionally, new strategies for diagnostic testing have been identified and are being implemented. These include creating EMR clinical care pathways and decision support systems, diagnostic test algorithms, and in-lab mechanisms for test sequencing. Significant benefits have been realized to date and opportunities still remain for the $\mathrm{CHCO}$ laboratory to improve the value of expensive send-out tests with active utilization management.

Development of a Preauthorization and Predetermination Process to Improve Access to Hereditary Cancer Risk Assessment Services and Subsequent Review of Reimbursement of CPT 96040 for Services Rendered

J. Polk ${ }^{1}$, P. Cirino ${ }^{1}$, J. Sales ${ }^{1}$, M. Borieux ${ }^{2}$

1. Hillcrest Hospital

2. Cleveland Clinic

In an effort to improve patient experience related to billing and reimbursement a multifaceted team was convened, including Hereditary Cancer Risk Assessment Genetic Counselor (GC), Oncology Business Manager, Oncology Financial Specialist (OFS), and Oncology Reimbursement Specialist (ORS) to establish a process for genetic counseling (GC) referrals and to determine and review reimbursement for GC services in an outpatient oncology 
clinic. A work flow process was developed. The referring provider enters a GC consult order in the electronic medical record, the order is routed to the OFS who contacts the patient's insurance for preauthorization and predetermination of benefits, and then the oncology department clerical team contacts the patient to schedule. Current billing practices at our institution include billing CPT 96040 as a facility charge, on a UB-04, utilizing the entity's tax ID. Following the GC consultation, the ORS reviewed each Explanation of Benefits (EOB) to determine the submitted charge less the contractual obligation in order to determine the allowed amount. A total of 153 claims were reviewed from a 9 month period (March 2013-November 2013). 111 of the claims accounted for 13 private commercial insurance payors. 8 payors had 2 or more claims and were included in the reimbursement analysis; 5 were excluded due to a being single claim. All state and federal payors (Medicare/ Medicaid) were excluded for the purposes of this analysis. Payors included in the analysis are noted as A-H with the following reimbursement represented as a percentage of the submitted charge. Company A $62 \%$, Company B $38 \%$, Company C $24 \%$, Company D $49 \%$, Company E $37 \%$, Company F $0 \%$, Company G $31 \%$, Company H $55 \%$. We are receiving reimbursement from $7 / 8$ companies analyzed and 3 are reimbursing at $49 \%$ or higher. Our review has determined that successful avenues for billing 96040 can be implemented.

\section{Cancer}

The Use of Diagnostic Exome Sequencing in the Identification of a Molecular Diagnosis in Cases Presenting with Cancer Phenotypes

C. Espenschied ${ }^{1}$, K. Farwell Gonzalez ${ }^{1}$, L. Shahmirzadi ${ }^{1}$, S. Gandomi ${ }^{1}$, D. El-Khechen ${ }^{1}$, Z. Powis ${ }^{1}$, C. Alamillo ${ }^{1}$, B. Tippin-Davis ${ }^{1}$, R. Baxter ${ }^{1}$, S. Tang ${ }^{1}$, E. Chao ${ }^{1}$

\section{Ambry Genetics}

As the use of diagnostic exome sequencing (DES) becomes more commonly integrated into the field of medical genetics, referral indications for DES will continue to expand. DES has been successful in providing an answer for 30-40\% of patients with undiagnosed Mendelian disorders and has the ability to identify novel genes not previously associated with human disease, including in cases of patients with cancer-related presentations. A retrospective analysis of the first 500 patients referred to our laboratory for DES testing revealed a total of 15 (3\%) patients with reported cancer-related clinical presentations. Of these 15 patients, at total of 4 (26.7\%) probands were found to have disease-causing alterations. Of these 4 patients, 1 (6.6\%) was positive for a mutation in a characterized gene $W N T 10 A$, a known gene not traditionally associated with cancer predisposition, while in 3 (20\%) likely pathogenic alterations were reported in novel genes (RAD54L ( 2 cases) and ETVO). The patient with the ETV6 alteration was also found to have an uncertain ARHGAP11A alteration, a gene which functions to stabilize p53. The 3 patients with novel gene findings presented with early-onset leukemia $(<2$ yo), paraganglioma-pheochromocytoma, and clear cell kidney cancer respectively. These cases also presented with other non-cancer related syndromic features, including alopecia, hyperpigmented skin lesions, high arched palate, anomalous dentition, and others. All of the patients with cancer phenotypes and a positive novel gene finding had previously tested negative for multiple single gene and/ or panel tests. This data suggests that the use of DES is a valuable and effective diagnostic testing option for the identification of both characterized and novel gene mutations in patients presenting with unique cancer phenotypes, including non-cancer related features, especially when other testing options have been exhausted. In addition, our data shows that novel gene findings were significantly more likely among patients with cancer-phenotypes than among other indications for testing.

\section{Germline BRCA Mutations in an Unselected Cohort of Patients with Pancreatic Adenocarcinoma}

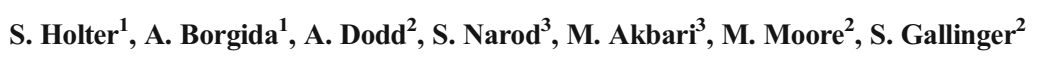

1. Mount Sinai Hospital

2. University Health Network

3. Women's College Hospital

This is the first prospective study to determine the frequency of $B R C A 1$ and $B R C A 2$ germline mutations in an unselected series of patients with pancreatic ductal adenocarcinoma (PDAC). Patients with histologically or clinically confirmed PDAC were approached at a single cancer center to enroll in the IRB-approved Ontario Pancreas Cancer Study. Patients provided informed consent and cancer family history data was obtained. Blood was collected and analyzed by Sanger sequencing and MLPA for germline variants in BRCA1 and BRCA2. Pathogenic germline mutations were identified in 14/307 (4.6\%) of unselected incident PDACs. BRCA1 mutations accounted for $1 \%(3 / 307)$ of the cases and BRCA2 for $3.6 \%$ (11/307). Average age of PDAC diagnosis was not significantly different between the BRCA mutation positive and negative cases, 62 years (42-78) and 65 years (27-91), respectively. Approximately $50 \%$ of each group was female. Ashkenazi Jewish ancestry accounted for $28 \%$ of the mutation carriers and $11 \%$ of the non-carriers. PDAC in another relative was reported in $11 \%$ of the negative cases and $20 \%$ of the positive cases (not significant). A personal history of previous primary cancer was significantly associated with BRCA mutation positivity; $50 \%$ in positive and $19 \%$ in negative $(p=0.007)$. A family history of breast and/or ovarian cancer was reported in $43 \%$ of the BRCA positive cases and $13 \%$ in the BRCA negative cases, this was statistically significant $(p=$ 0.005 ). In both groups, $1 / 3$ of those with a family history of breast and/or ovarian cancer did not meet provincial guidelines to warrant BRCA analysis. Previous studies of BRCA mutations in PDAC have been limited to retrospective case series and incomplete germline analysis. Our study shows that germline BRCA mutations account for a significant proportion of PDAC, regardless of family history of breast and/or ovarian cancer. Increased identification of PDAC patients with germline BRCA mutations may allow for tailored chemotherapy through the use of platinum agents or PARP inhibitors.

Large Scale Changes in Cancer Genetic Testing with Variable Integration of Expanded Gene Panels

G. Hooker' ${ }^{1}$, A. Buchanan ${ }^{2}$, K. Rhoads ${ }^{2}$, K. J. Vogel Postula ${ }^{3}$, J. Quillin ${ }^{4}$, P. Summerour ${ }^{5}$, R. Nagy ${ }^{6}$

1. $N \operatorname{ext} G x D x$

2. Duke University Medical Center 
3. GeneDx

4. Virginia Commonwealth University

5. Ambry Genetics

6. Ohio State University

The NSGC Cancer Special Interest Group (SIG) conducted a survey of its membership in November 2013 to capture the clinical impact of both the 2013 Association for Molecular Pathology (AMP), et al. v. Myriad Genetics, Inc., et al. Supreme court decision and the introduction of cancer genetic testing panels. We set out to describe genetic counselors' experiences with the changing marketplace for genetic tests for Hereditary Breast and/or Ovarian Cancer (HBOC). Anonymous online surveys were completed by 157 genetic counselors from the NSGC Cancer SIG. The survey included four hypothetical patients, asking respondents which tests they may have considered prior to the AMP v. Myriad decision and the availability of panel-based testing and which tests they may currently consider. We also asked participants about changes to their practice and testing patterns. Items were developed by expert consensus and a brief pilot. Most (93\%) of counselors reported changing the types of tests they order for HBOC over the last 12 months, and $84 \%$ reported a different preference for first line testing prior to these developments vs. currently, for at least one of the four vignettes. We conducted bivariate analyses with one-way ANOVA to identify correlates of changes in ordering and found variation by NSGC geographic region in both selfassessments of change ( $\mathrm{F} 5,147=6.2, p<.01)$ and changes in preference for first-line hypothetical testing ( $\mathrm{F} 5,143=3.1, p=.01)$, with Region 6 reporting the most change and Region 2 reporting the least change in testing preferences. Among counselors' selecting expanded gene panel tests for their current first-line hypothetical test choice, there was variation in types of panels preferred (e.g. breast cancer-specific vs. general cancer panels). This study found significant changes in genetic testing practices among cancer genetic counselors in the past year, with wide variation in testing preferences. Our findings provide a valuable summary of current clinical practice and highlight research priorities regarding the implementation of expanded genetic testing panels for cancer risk assessment.

Cancers Associated with BRCA1 and BRCA2 Mutations Other Than Breast and Ovarian

J. Mersch ${ }^{1}$, M. Jackson ${ }^{2}$, C. Singletary ${ }^{1}$, S. Peterson ${ }^{2}$, D. Nebgen ${ }^{2}$, M. Park², J. Litton ${ }^{2}$

1. The University of Texas Health Science Center at Houston

2. The University of Texas MD Anderson Cancer Center

Mutations in BRCA1 and BRCA2 cause tumor development in Hereditary Breast and Ovarian Cancer syndrome (HBOC) through accumulation of unrepaired DNA damage. Extensive research of $B R C A 1$ and $B R C A 2$ mutations has led to well-defined breast and ovarian cancer risks in individuals with HBOC. Previous studies have reported additional cancers associated with BRCA mutations; however, the type of cancer, magnitude of risk, and differences between sexes remains to be clarified. Ultimately, a consensus of additional cancer risks can aid in better recommendations for genetic testing and more effective screening and prevention guidelines. A retrospective chart review of University of Texas MD Anderson Cancer Center patients identified 1,081 individuals with a BRCA mutation. A detailed cancer history for each person was collected and compared to the general population incidence rates reported by the $\mathrm{CDC}$ using standardized incidence ratios (SIR). Individuals with a $B R C A 1$ mutation did not have a significant increase in other cancers; however, a trend toward an increased risk for melanoma was observed in men and women. Individuals with a $B R C A 2$ mutation had significantly higher number of observed cases compared to expected cases for pancreatic cancer (SIR 21.7, $95 \%$ CI 13.1-34.0, $p$-value $<0.001$ ) in both men and women and prostate cancer in men (SIR 4.9, $95 \%$ CI 2.0-10.1, $p$-value $=0.002$ ). The results of this study uphold the current recommendations for HBOC screening by the National Comprehensive Cancer Network; however, the high rate of pancreatic cancer in our study sample emphasizes the need to develop effective screening for individuals at increased risk. Additional studies are being conducted to evaluate other cancers in families with BRCA mutations.

\section{Reasons Patients Do Not Pursue BRCA Genetic Testing following Genetic Counseling}

\section{Raymond ${ }^{1}$, S. Hayden ${ }^{2}$, S. Mange ${ }^{3}$, N. Petrucelli-Walden ${ }^{4}$}

\section{University of Michigan}

2. MidMichigan Medical Center

3. Michigan Department of Public Health

4. Karmanos Cancer Institute

Introduction: Genetic counseling and testing can identify high-risk individuals who may benefit from enhanced cancer surveillance and prevention programs. However, not all individuals undergo genetic testing following genetic counseling. Understanding the reasons for not testing is necessary for the clinical integration of genetic testing. Methods: A collaborative project through the Michigan Department of Community Health collects anonymous data on BRCA-related genetic counseling visits performed by genetics providers in Michigan. These data include demographics, patient and family cancer history, genetic test results and reasons for declining testing. Results: From 2008 to 2012, 10,726 patients $(94.8 \%$ female) underwent genetic counseling; 3,476 (32.4\%) patients did not pursue genetic testing. Untested individuals were less likely to have breast and/or ovarian cancer $(p<0.01)$; less likely to be of Ashkenazi Jewish ancestry $(p<0.01)$ and more likely to be female $(p<0.01)$. The top reasons for not testing included: patient was not the best test candidate $(26.8 \%)$, testing was not clinically indicated $(22.2 \%)$ and insurance coverage was inadequate (13\%). Among untested individuals with breast and/or ovarian cancer, $22.2 \%$ listed inadequate insurance coverage as the primary reason testing was not pursued and $6.6 \%$ listed failure to meet Medicare criteria. $12.7 \%$ of the 2,671 untested individuals with private insurance listed inadequate coverage as the primary reason for not testing. $17.2 \%$ of untested patients declined testing for reasons indicating a personal patient choice. Conclusion: In a 5 year time period, over one-third of patients who underwent BRCA genetic counseling did not pursue genetic testing. In almost half of these patients, genetic testing was not indicated, either based on risk assessment or because they were not the best test candidate. However, inadequate insurance coverage continues to be a barrier to appropriate genetic testing, even for those affected with cancer and those with private insurance. 
Physician Experiences and Understanding of a Genomic Sequencing Project for Oncology Patients

C. Weipert ${ }^{1}$, K. Ryan ${ }^{2}$, J. Everett ${ }^{3}$, B. Yashar ${ }^{4}$, R. De Vries ${ }^{2}$, B. Zikmund-Fisher ${ }^{5}$, V. Raymond ${ }^{3}$

1. University of Michigan

2. Center for Bioethics and Social Sciences in Medicine, University of Michigan

3. Cancer Genetics Clinic, University of Michigan

4. Department of Human Genetics, University of Michigan

5. University of Michigan School of Public Health

Introduction: Although use of genomic technologies in oncology is expanding, questions remain about incorporating sequencing results into clinical practice. Studies examining physician perspectives on genomic sequencing (GS), including incidental germline (IGL) findings, are an essential step in learning how to tailor genomic information for clinical use. Methods: We conducted 17 semi-structured interviews from 11/13-1/14 with physicians who had referred patients to a tumor analysis study with the goal of identifying genetic mutations that could inform therapeutic choices. Interviews focused on: 1) cancer treatment decisions; 2) gene mutations/GS; and 3) expectations for/communication of results. Interviews were audio recorded and transcribed. A codebook was created using a stepwise process: 1) identification of global themes, 2) description of trends within themes, and 3) iterative analysis on all transcripts using Dedoose data analysis software. Results: Of physicians interviewed, $88 \%$ worked at academic centers; $29 \%$ held MD/PhDs; and $53 \%$ finished medical school $<20$ years ago. Thirty-five percent lacked understanding of the technology, $35 \%$ indicated a need for more genetics education, and $41 \%$ felt academic oncologists would have higher levels of genetic knowledge than those in the community. Twenty-nine percent lacked clarity about potential types of IGL findings, $65 \%$ expressed discomfort in disclosure of IGL results to patients, and $71 \%$ felt genetic counselors were needed when IGL results were found. Conclusions: One-third of physicians referring patients to a GS study lacked understanding of the sequencing technology and almost half felt academic oncologists would have higher levels of understanding than those in the community, suggesting that more consistent genetics education efforts are needed. Physician-identified challenges related to IGL findings signal a need for structural solutions, such as the involvement of genetic counselors on similar sequencing projects, a role that represents a potential area of expansion for the genetic counseling field.

\title{
Education/ELSI
}

\author{
Assessment of the Readability of Genetic Counseling Patient Letters \\ E. Brown ${ }^{1}$, M. Skinner ${ }^{1}$, S. Ashley ${ }^{1}$, K. Reed ${ }^{2}$, S. Delany Dixon ${ }^{3}$ \\ 1. University of Maryland \\ 2. The Jackson Laboratory \\ 3. University of Maryland School of Medicine
}

Patient letters are a powerful tool that genetic counselors use to communicate with their patients. Patient letters are often sent to provide information on a new diagnosis, reiterate test results, and to serve as a permanent record of the visit. Patient letters, however, are only helpful if the patients can understand them. More than $50 \%$ of the U.S. population reads below a 9 th grade reading level and over a third of the population has low health literacy skills. In this study we evaluate the readability of genetic counseling patient letters by assessing reading level, image use, and terminology use. One hundred forty nine genetic counselors participated in the survey and 79 submitted a sample patient letter. Analyses of the letters revealed a mean reading level of 10.93 . On average, five genetic terms were included in each letter, and only $20 \%$ of these terms were defined. Analyses of survey responses revealed over $75 \%$ of genetic counselors do not include images in their patient letters. These results indicate there is room for improvement in order to make genetic counseling patient letters more accessible to the general population.

\section{Genetic Counseling Student Experiences of Mental Health Concerns}

\author{
A. Cantor ${ }^{1}$, C. Hippman ${ }^{2}$, L. Hercher ${ }^{1}$, J. Austin ${ }^{3}$
}

1. Sarah Lawrence College

2. BC Women's Hospital and Health Centre

3. University of British Columbia

Introduction: As many as $50 \%$ of undergraduates, graduates and medical students experience mental illness. For these students, mental illness poses significant risks to academic productivity and future employment, as well as to their social relationships and, most worryingly, an increased risk for suicide. However, there is limited research on the mental health of students in professional graduate programs, including genetic counseling. Purpose: We sought to explore self-reported rates of mental illness during the genetic counseling program and help-seeking behaviors among genetic counseling students and recent graduates. Methods: We distributed an online survey to assess respondents' personal experiences of mental illness during their genetic counseling training via NSGC discussion forums, NSGC SIGs, and genetic counseling training program directors. Responses were eligible for inclusion in the descriptive analysis if the respondent was a current student in an accredited genetic counseling training program or had graduated from one within the past 5 years. Results: Between January 27 and February 3, 2014, 227 eligible completed surveys were received, including 149 (67\%) from current students and $75(32 \%)$ from recent graduates. There were $68(30 \%)$ who reported having an episode of mental illness during their genetic counseling training, 22 (32\%) of whom reported that this was their first experience of mental illness symptoms. Of the 134 (59\%) who reported having ever experienced mental illness in their lifetime, 39 (29\%) reported having never sought treatment for mental illness. Conclusions: Our data suggest that episodes of mental illness are common among genetic counseling students during training. Though there is potential for ascertainment bias, our findings are consistent with studies of other student populations. Our data, in combination with existing literature, highlight the need for genetic counseling programs to identify students experiencing mental health concerns and to develop strategies to support students' mental health during their training. 


\title{
Moving Beyond Likert Scales: Competency-Based Milestones and Implications for Genetic Counseling Education
}

\section{Guy ${ }^{1}$}

\section{University of Oklahoma Health Science Center}

Graduate medical education has increasingly focused on the development of medical specialty milestones to provide a systematic tool for medical resident evaluation. In the field of Medical Genetics, the Milestones have been designed to provide a framework for assessment of the development of a resident over the course of training, while also providing feedback on the educational effectiveness of residency programs. Many genetic counseling programs are housed within College of Medicine programs and have faculty with dual roles in genetics residency programs and genetic counseling programs. Genetic counseling educators in such programs may wish to explore the utility of the development of genetic counseling milestones for evaluation of genetic counseling students. As this area of graduate medical education research develops, it is an optimal time for genetic counseling educators to explore this concept. The development of Genetic Counseling Milestones may provide a valuable tool to assess genetic counseling students during their education. However, we must first determine if the development of such a tool would provide a significant improvement to current evaluation methods, without placing undue burden on genetic counseling programs. To explore this, it is necessary for genetic counseling educators to have a thorough understanding of the educational theory and practical implications of the implementation of competency-based milestones. The genetic counseling and medical education literature review presented here provides foundational knowledge of the creation and programmatic structure of competency-based milestones in medical residency programs and the analysis of outcome data from medical specialty milestone implementation. Thus, providing the first step in the exploration of the utility of Genetic Counseling Milestones in the education of genetic counseling students.

\section{Video Patient Encounter for Genetic Counsellor Skill Development}

\author{
J. Scott' ${ }^{1}$, T. $\mathbf{O h}^{2}$ \\ 1. University of British Columbia \\ 2. BC Children's and Women's Hospital
}

Facilitating decision making is a key component of genetic counselling. The American Board of Genetic Counseling and the Canadian Association of Genetic Counsellors recognize this skill as a core competency and masters programs in genetic counselling accredited by the Accreditation Council of Genetic Counselors have developed various strategies to develop this skill in their curriculum. Traditionally, facilitating decision making has been taught through seminars, role play scenarios, and eventually supervised patient encounters in the clinical setting. Some programs have developed standardized patient encounters, though the costs can be prohibitive and this has not become standard practice across institutions. There is an increasing availability of technology for use in the educational setting including virtual patients, and video patient encounters. While development of these tools is costly, sharing the finished product is easy and inexpensive. We developed and piloted a video patient encounter and facilitator manual for use in genetic counselor education for safe development of this critical skill. The format is akin to a "Choose your own ending" story. The video encounter includes details on the reason for the referral, video clips on contracting and preparation for decision making, followed by five video clips showing different methods for facilitating decision making. The learner is intended to discuss and role play possible strategies for facilitating decision making prior to viewing an example of the method as demonstrated by an expert genetic counsellor. The video patient encounter was piloted and evaluated by recent graduates and current students in genetic counselling programs and shown to be an effective method for advanced genetic counselling skill development. Further development and sharing of technology enabled learning resources between genetic counselling programs may assist programs with expansion in class size without increased burden on existing clinical supervisor resources.

\section{Balancing Cost Management and Ethics: A Proposed Process for Inpatient Genetic Testing Appraisal for Atypical Cases}

\section{Smith ${ }^{1}$, T. Moss ${ }^{1}$}

\section{Cleveland Clinic}

Regulations on inpatient genetic testing are growing due to institutional cost repositioning. The following is a complex case illustrating how a challenging social situation factored into genetic testing decisions. Prenatal genetics was consulted for an inpatient 22yo G1P0 at $22 \mathrm{w} 1 \mathrm{~d}$ due to pulmonary hypertension (PHTN) diagnosed at age 11. Paternal family history was positive for an aunt diagnosed at 25 (deceased), first cousin at 19 (deceased), and aunt in her 30s (surviving, no contact). Patient was admitted at 21 weeks; based on worsening symptoms, she was deemed unlikely to survive delivery. Testing of BMPR2 with reflex to other PHTN genes was ordered by genetics so that site-specific analysis would be possible for the child, but was flagged by Lab Medicine. At our center, inpatient genetic testing is to only be used when it will directly impact immediate patient care due to cost/reimbursement considerations. A written case was made to the Bioethics, Financial, Legal, and Molecular Genetics teams detailing how testing would impact family healthcare considering the patient's poor prognosis. All teams were in favor of testing the patient; Lab Medicine then supported testing. A BMPR2 mutation was identified, c.1471C $>$ T (p.R491W). Cesarean Section (CS) was completed at 23 w4d due to worsening symptoms; the patient never recovered (removed from mechanical ventilation 3 days post CS). After the patient's death, her daughter became a ward of the state. With this child likely to enter into foster care/adoption, concern was raised about lack of follow-up. Multidisciplinary consensus agreed to test the baby as an inpatient; Lab Medicine then approved. Inpatient testing restrictions are necessary to ensure wise use of healthcare dollars, but unique circumstances arise which merit bending of the rules. This case led to the development of a multidisciplinary team including Financial, Legal, Ethics, and Molecular Genetics for submission of future requests. This case example and our adopted protocol can serve as a model and discussion catalyst for other institutions. 
Informed Consent in Pediatric Genome-Wide Sequencing: Content of Informed Consent Sessions and Parental Understanding

\author{
S. Wiley ${ }^{1}$, A. Tomlinson ${ }^{2}$, L. Conway ${ }^{1}$, C. Ulrich ${ }^{2}$, B. Bernhardt ${ }^{2}$ \\ 1. Genetic Counseling Program, Arcadia University \\ 2. Perelman School of Medicine, University of Pennsylvania
}

\begin{abstract}
Although whole exome sequencing (WES) and whole genome sequencing are offered increasingly in pediatric settings, there are few guidelines for informed consent (IC). Accordingly, we assessed the extent to which important elements of IC were included in audio-taped IC sessions conducted by 10 clinicians with families enrolled in an NIH-funded Clinical Sequencing Exploratory Research project. We developed a checklist of 32 important elements of IC and assessed whether each element was discussed in 40 sessions. Parents of participating children were surveyed 3 days after the IC session to assess understanding of WES and study procedures. We used chi-square analysis to determine whether inclusion of an element in a session was associated with parental understanding. Results showed sessions included mention of $62.7 \%$ of the IC elements. Certain items were discussed in nearly all sessions, like the possibility of finding the cause of the child's condition, and that immediately medically actionable results would be automatically returned. Other items were mentioned rarely, including risks to confidentiality and lack of direct benefit from participation. Parents correctly answered $78 \%$ of items on the understanding survey. Nearly all parents understood the benefits of the study and study procedures. Only a minority of parents understood the risks to confidentiality and the types of incidental findings that would be released. Paired analyses of inclusion of 19 IC elements and correct understanding showed no statistically significant associations for any element. These data document clear gaps in families' understanding of participation in genomic sequencing research. The lack of an association between provider discussion and parental understanding of important elements of IC implies there are other factors relating to parental understanding beyond the IC discussion. Additional research is needed to identify ways to improve participant understanding of genomic research and the risks, benefits and limitations of genomic sequencing.
\end{abstract}

\title{
GC Professional Roles
}

Emerging Genetic Counselor Roles within the Biotechnology and Pharmaceutical Industries: As Industry Interest Grows in Rare Genetic Disorders - How Are Genetic Counselors Joining the Discussion?

\author{
T. Field ${ }^{1}$, S. Brewster ${ }^{2}$, M. Towne ${ }^{3}$, M. Campion ${ }^{1}$ \\ 1. Boston University School of Medicine \\ 2. Translational Neuroscience Center, Boston Children's Hospital \\ 3. Boston Children's Hospital and Harvard Medical Center
}

A recent trend in the biotechnology and pharmaceutical industry (BPI) is the development of therapies for rare or orphan disorders including many genetic disorders - offering an emerging career for Genetic Counselors (GCs) within BPI. Orphan drug development requires navigating genomic communication with an understanding of the community's needs, and translating research, clinical trial information, and genetic concepts to clinical and non-clinical audiences. To date there is limited data regarding GCs working in BPI. This study aimed to define the roles and motivation of GCs in BPI, assess job satisfaction, and identify translatable skills and current gaps in GC training programs. Study participants were identified through referral sampling $(n=40)$ and invited to an online anonymous survey. An invitation was also sent to the National Society of Genetic Counselors (NSGC) membership. We identified 26 GCs working in BPI in the United States. GC positions in BPI are growing $-57 \%$ of respondents were the first GC in their role. $79 \%$ worked for companies focused on rare disorders. GCs in BPI use core genetic counseling competencies; education and professional practice domains are used most frequently. However, $72 \%$ felt their training did not fully prepare them for BPI, especially in the areas of business development (93\%), drug development (67\%), and clinical trials knowledge (67\%). Satisfaction of GCs in BPI was compared to reported satisfaction in the NSGC's Professional Status Survey (PSS). While we lacked sample power to reach significance, satisfaction of GCs in BPI was consistently higher than PSS respondents in the areas of salary (BPI mean=2.32, PSS clinic GC mean=1.72) and advancement opportunities (BPI mean=2.05, PSS clinic GC mean 1.45 ) - reasons commonly sited for leaving the profession. These data support emerging GC roles in BPI as interest in rare disorders continues to grow. Offering exposure to BPI in GC training programs would better prepare future generations of GCs to not only join, but lead, the discussion of drug development for rare disorders.

The Expanding Role of Genetic Counselors in Industry-Based Employment and Emerging Professional Issues: The Intersection of Innovation and Conflicts-of-Interest

\author{
1. Ambry Genetics \\ 2. Feinberg School of Medicine, Northwestern University \\ 3. Ariosa Diagnostics \\ 4. Eli Lilly and Company \\ 5. Verinata Health \\ 6. Recombine \\ 7. $N \operatorname{ext} G x D x$
}

S. Gandomi ${ }^{1}$, K. Farwell Gonzalez ${ }^{1}$, S. Aufox ${ }^{2}$, A. Batey ${ }^{3}$, S. Prucka ${ }^{4}$, H. Snyder ${ }^{5}$, J. Kaplan ${ }^{3}$, N. Kumar ${ }^{6}$, G. Hooker ${ }^{7}$

Recently, there has been an exponential growth of industry-based employment opportunities for genetic counselors. With this growth comes diversification of roles for genetic counselors working in the private sector, and specifically an increased number of them seeking to apply their research and patient advocacy skill sets outside of a traditional academic model. As public-private research partnerships and efforts to collect and share data become more prevalent, genetic counselor involvement becomes more relevant. Genetic counselors are uniquely positioned to address issues such as 
genetic privacy, returning of research results, and ethical considerations for data collection and sharing both within and across industry and academia. Concurrently, it is also important to recognize the challenges faced by genetic counselors involved in private sector research, including appropriate identification and disclosure of conflicts of interest, working with commercial Institutional review boards and managing collaborations within or outside of industry. We aim to review key issues related to research in industry, evaluate existing models and practices in other professional organizations, and highlight our ongoing efforts to both educate counselors on these issues and foster an open exchange of experiences and ideas from both clinical and industry based counselors engaging in research. Additionally, we suggest opportunities to support growth in this area through SIG-sponsored events, informational webinars, publications and trainings. Sharing varied perspectives allows our profession to have a more robust understanding and ability to provide a unique voice to professionals exploring opportunities in this space. It is well established that genetic counselors are playing a major role in the implementation of genomic innovations. In promoting thoughtful discussion and consideration of issues arising at the intersection of innovation and conflicts of interest, we aim to promote growth of our field and our role in promoting the effective integration of genomic medicine.

\title{
Development, Experience and Expression of Meaning in Genetic Counselors' Lives: An Exploratory Analysis
}

\author{
D. Wells ${ }^{1}$, P. McCarthy-Veach ${ }^{1}$, D. Cooper ${ }^{1}$, L. Benolken ${ }^{1}$, B. LeRoy ${ }^{1}$
}

\section{University of Minnesota}

Genetic counselors routinely engage with patients and families grappling with questions of meaning when making decisions about genetic risk. Research and theory demonstrate genetic counselors gain important personal insights through their work and grow professionally from self-reflective practice regarding their beliefs and values. Data are lacking, however, about the nature of the meaning genetic counselors bring to the profession and how they directly experience and/or navigate issues of meaning within clinical practice. Accordingly, a national sample $(N=298)$ of practicing genetic counselors completed a survey assessing demographic characteristics and willingness to participate in a semi-structured telephone interview exploring their views on meaning as they relate to their clinical work and professional development. Sixty-eight individuals of varied experience levels were interviewed about: 1) how they define a meaningful life for themselves; 2) lifetime sources of influence on their sense of meaning; 3) work-related contexts that reaffirm their sense of meaning; 4) work-related contexts that challenge their sense of meaning; 5) how their sense of meaning has changed over time; and 6) reasons for participating in this study. Twenty-five interviews were analyzed using Consensual Qualitative Research methods; data saturation was reached at that point. Thirty-six thematic domains and 31 categories were extracted. Common themes included: importance of satisfying relationships; helping others; personal fulfillment; personal and patient experiences of illness and loss; religious and/or spiritual foundations; value conflicts; competing obligations; challenges to meaning; development of empathy; resiliency; and increased humility. Results suggest the importance of professional venues for discussions of meaning (e.g., genetic counseling program curricula, continuing education, peer supervision/consultation). Additional findings, illustrative examples, and practice and research implications are presented.

\section{Career Advancement in Genetic Counseling: Perceived Opportunities and Barriers among Practicing Genetic Counselors}

\author{
N. Poullard ${ }^{1}$, M. Hardy ${ }^{2}$, E. Williams ${ }^{3}$, N. Callanan ${ }^{1}$ \\ 1. University of North Carolina at Greensboro \\ 2. The Perinatal Center at Henrico Doctor's Hospital \\ 3. Labcorp
}

The NSGC Professional Status Survey reveals that while genetic counselors are satisfied with many aspects of the career, they are less satisfied with opportunities for advancement. Our goal was to assess genetic counselors' perceptions of career advancement including perceived opportunities and barriers. An anonymous online survey was distributed to NSGC members. Of the 468 participants, 373 (77.5\%) completed the entire survey. The Professional Excellence and Career Advancement in Nursing (PECAN) model was used as a career advancement paradigm for genetic counseling to examine factors that may contribute to or hinder career advancement. The model recognizes that advancement is influenced by several factors, including human capital (self-efficacy), social capital (mentorship), system (work environment) and external support (family/friends). Participant definitions of career advancement included assuming new or additional responsibilities $(71.5 \%)$, salary increases $(49.5 \%)$ and promotions $(41.2 \%)$. Although $69 \%$ of participants indicated that they had advanced in their careers, more clinical counselors reported that they had not advanced (Fisher's exact test, $p$ value $=.009)$. Overall, participants perceived that there are limited opportunities for advancement $(61 \%)$ and that counselors in industry $(77 \%)$ or nonclinical positions $(72 \%)$ have more advancement opportunities. The most common barriers to advancement included a "lack of structured advancement opportunities" (73 \%) and a "lack of knowledge from supervisors on how to use my skills" (44\%). While the majority of participants had advanced in their careers, advancement opportunities for clinical genetic counselors were perceived as limited. Genetic counselors perceived that organizational barriers create a ceiling effect in the clinical realm that can often only be overcome by moving to industry or non-clinical settings. We propose that, in order to address this concern within the field, genetic counselors can establish a standardized career ladder that can be applied to a variety of settings.

\section{The Genetic Counseling Assistant: Is Our Profession Ready for Multiple Career Levels?}

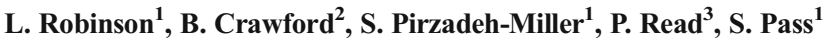 \\ 1. Simmons Cancer Center, The University of Texas Southwestern Medical Center \\ 2. Cancer Risk Program, University of California San Francisco \\ 3. The University of Texas Southwestern Medical Center
}

As the role of the genetic counselor continues to expand and develop it imperative that the field recognize the need to maximize the skill set of certified genetic counselors by employing qualified assistants. Several academic institutes created a position entitled the genetic counseling assistant or GCA, and 
have developed a service delivery model that maximizes the training of a certified genetic counselor and allows for the GCA to acquire real life experiences to prepare themselves for the field of genetic counseling. We will discuss how this new position was developed within two healthcare system. The job responsibilities of the GCA will be outlined. GCA are currently used to call out negative test results, write routine correspondence, obtain insurance authorizations and write letters of medical necessity for testing. They are also used to assist in research studies and in clinics. GCAs have a background in science and a bachelor's degree and are interested in a graduate education in genetics. Program directors and college professors have been excellent resources for possible candidates. This position gives extensive real life experience about our profession. We will review the financial benefits of the assistants to our program's overall vision and the ideal ratio of genetic assistant to genetic counselors. The response from our hospital's administration about the change in program structure with the GCA will be discussed. We will look at the positive and negative responses from the genetic counselors that have worked with a GCA in regards to their job satisfaction before and after the role was introduced, and from the GCAs that have been in this position. Historically other professions have developed into multiple career levels, like nurses and physical therapists. Is it time of the genetic counseling community to evolve into a tiered profession? If so, should our society help mold the qualification of a GCA?

\section{Genetic Counseling Licensure: An Oral History}

K. Valverde ${ }^{1}$, K. Nugent ${ }^{1}$, L. Conway ${ }^{1}$

\section{Arcadia University}

Many professions are regulated by states using licensure, which provides legal recognition that an individual is qualified to practice in a profession. Until the early 2000's, the profession of genetic counseling was not licensed in any state. The first legislation was passed by California, and the first licenses were issued by Utah in 2001. Over the past 13 years, 32 states plus the District of Columbia have taken formal steps toward attaining genetic counseling licensure. This project aims to document and preserve the process of obtaining licensure for genetic counselors. Interviews were conducted with 16 key informants from 13 states in various stages of the licensure process. Interviews were transcribed verbatim, and analyzed by two coders (KN and KV) using NVivo 10 software. Several topics were frequently discussed, including the value of patient testimony $(n=6)$, use of lobbyists ( $n=8)$, use of NSGC licensure materials $(n=7)$, timing the introduction of the bill $(n=6)$, compromising on the language of the bill $(n=8)$, avoiding contentious topics $(n=3)$, and conducting due diligence $(n=6)$. Factors associated with successful attainment of licensure were a favorable political climate, correct political connections, and access to the proper resources. Common obstacles that state informants encountered were lack of documentation proving patient harm by unqualified genetic counselors $(n=6)$ and an unfavorable political climate $(n=5)$. All participants indicated they felt that licensure is important for validating genetic counseling as a respected medical profession. Documenting the experience of successful as well as unsuccessful states has shed light on critical aspects of the legislative process. Additional interviews with individuals in other states may identify other factors that need to be considered as more states develop legislation. Future work will include a survey of licensed genetic counselors to identify whether and how practice has changed with the implementation of licensure.

\section{Genetic Testing I: New Technology}

\section{Attitudes of Genetic Counselors toward Return of Incidental Results from Exome and Whole Genome Sequencing}

M. J. Bamshad', S. M. Jamal' ${ }^{1}$, T. Harrell ${ }^{1}$, H. K. Tabor ${ }^{2}$, J. H. Yu ${ }^{1}$

1. University of Washington

2. Seattle Children's Research Institute

Introduction: Professional recommendations for return of results from exome and whole genome sequencing (ES/WGS) have been controversial. The lack of clear guidance about whether, and if so, how to return ES/WGS incidental results limits the extent to which individuals/families may benefit. Genetics professionals' perspectives, particularly those of genetic counselors who are at the forefront of using clinical ES/WGS, are largely unknown. Empirical data on such stakeholder perspectives could help clarify how to weigh expert positions and recommendations. Hypothesis: Genetic counselors hold the position that a limited number of actionable incidental findings for severe conditions from ES/WGS should be offered to patients. Methods: We conducted an online survey from Aug-Sept 2013 of 9,857 genetic professionals to learn their attitudes on return of incidental ES/WGS results and recent American College of Medical Genetics and Genomics (ACMG) recommendations for reporting of incidental findings in clinical ES/WGS. Results: The survey was completed by 167 genetic counselors. Nearly all thought that incidental ES/WGS results should be offered to adult patients (95\%), healthy adults $(79 \%)$, and parents of a child with a medical condition (75\%). Of note, more than a third disagreed that incidental results offered should be limited to those that are actionable. About half (48\%) thought that incidental results about adult-onset conditions should be offered to parents of a child with a medical condition, whereas the majority thought carrier status (60\%) and childhood-onset conditions (94\%) should be offered. Most (74 \%) disagreed that healthcare providers should decide which incidental results should be returned, but rather thought that patient/family preferences should guide return. Most (73\%) disagreed that incidental results should be reported to families irrespective of their preferences. Conclusions: Genetic counselors' perspectives on return of ES/WGS results differ from current recommendations and underscore the need for including stakeholder perspectives in developing recommendations for return of incidental results.

Consumers Report Lowered Confidence in Their Genetics Knowledge following Personal Genomic Testing: Findings from the PGen Study

D. A. Carere' ${ }^{1}$ T. Moreno ${ }^{2}$, J. Mountain ${ }^{3}$, J. S. Roberts ${ }^{4}$, R. C. Green ${ }^{5,6}$

1. Harvard School of Public Health

2. Pathway Genomics Corporation

3. 23andMe Inc.

4. University of Michigan School of Public Health 
5. Harvard Medical School

6. Brigham and Women's Hospital

Background: Commercial personal genomic testing (PGT) places high demands on consumer genetic literacy; it may, however, also serve as an opportunity for genetics education. We measured two components of genetic literacy among participants in a longitudinal study of 23 andMe and Pathway Genomics customers. We hypothesized that participants would show improved genetic knowledge (GK) and self-efficacy with genetics concepts (GSE) following PGT. Methods: At both baseline (BL, pre-results) and 6 months post-results (6 M), GK was measured with 9 true/false statements, and GSE was measured by agreement with five statements on a 7-point Likert scale (e.g., I am able to explain to others how genes affect one's health). Our hypothesis was evaluated with t-tests and chi-squared tests. Results: Longitudinal data was available from 900 participants in the Impact of Personal Genomics (PGen) Study (mean age $=46.1$ years; $59.2 \%$ female; $80 \%$ college-educated). Mean GK was $>8$ out of 9 at both BL and $6 \mathrm{M}$. Performance was poorest on a question regarding genetic disease etiology (63.4\% correct). Mean GSE was moderate at both BL (29.1 \pm 5.5 out of 45$)$ and $6 \mathrm{M}(27.7 \pm 5.5)$, but the proportion of participants that agreed/strongly agreed with each statement showed substantial, and statistically significant $(p<.0001)$ decreases on 4 of 5 statements. For example, $82.3 \%$ stated at BL that they were "able to understand information about how genes can affect [their] health," but only $64.6 \%$ expressed similar confidence at $6 \mathrm{M}$. Participants were least confident in their ability to explain health genetics to others $(50.2 \%$ agreed/strongly agreed at BL; $38.6 \%$ at $6 \mathrm{M})$. Conclusion: In a highly educated population of PGT users, objectively measured genetics knowledge was high and did not change after testing; however, users showed significantly reduced confidence in applying genetics knowledge postPGT. PGT may impact self-efficacy by revealing the complexity of health genetics; genetic counselors should be mindful of both this effect, and the implications of how genetic literacy is conceptualized and measured.

Revised Diagnosis through Exome Sequencing of an Infant with Congenital Cataracts Expands Phenotypic Spectrum of COL4A1-Associated Disorders

G. Chandratillake ${ }^{1}$, S. Garcia ${ }^{1}$, J. Tirch ${ }^{1}$, J. Harris ${ }^{1}$, A. Patwardhan ${ }^{1}$, S. Chervitz ${ }^{1}$, B. Mendelsohn ${ }^{2}$, J. West ${ }^{1}$, R. Chen ${ }^{1}$, A. Slavotinek ${ }^{2}$

1. Personalis, Inc.

2. University of California San Francisco Benioff Children's Hospital

An infant presented for ophthalmologic examination due to an abnormal red reflex on exam in the newborn period. The infant was diagnosed with congenital cataracts and scheduled for lensectomy at 1 month of age. During surgery, the child underwent a cardiac arrest, was resuscitated, but ultimately sustained severe organ damage such that the parents opted for comfort care. In addition to the congenital cataracts that had prompted surgery, several other features were revealed by autopsy: hypertrophic cardiomyopathy and chronic metabolic myopathy with abnormal mitochondria, and multifocal intraparenchymal hemorrhages. The latter were interpreted as hypoxic-ischemic encephalopathy secondary to the cardiac arrest, and the infant was diagnosed with Sengers syndrome due to the presence of congenital cataracts together with hypertrophic cardiomyopathy and metabolic myopathy. Sengers syndrome, characterized by congenital cataracts, hypertrophic cardiomyopathy, skeletal myopathy, exercise intolerance, and lactic acidosis, is an autosomal recessive disorder caused by loss-of-function variants in the gene encoding mitochondrial acylglycerol kinase, $A G K$. $A G K$ sequencing was performed for this infant, but no pathogenic variants were found. Whole exome sequencing was then performed on a research protocol on the trio (proband and parents), revealing a de novo missense variant, p.Gly773Arg, in COL4A1 in the proband. COL4A1 encodes the alpha-1 subunit of collagen type IV, which plays an integral role in the structure of basement membranes. Heterozygous variants in COL4AI are associated with a diverse range of phenotypes, reflecting the gene product's structural role in connective tissue. Relevant to this case, variants in the gene have been observed to cause prenatal onset intracranial hemorrhage, myopathy, and in a few reported cases, congenital cataracts. In conclusion, exome sequencing facilitated a revised diagnosis, which expands the phenotypic spectrum of COL4A1-associated disorders. The revised diagnosis also changed the assessment of recurrence risk for this family.

Maximizing the Effectiveness of Exome Testing: A Retrospective Comparison of Diagnostic Yield in Singletons versus Multiple Family Members in over 1,500 Cases Submitted for Whole Exome Sequencing

H. Hanson Pierce ${ }^{1}$, J. Neidich ${ }^{1}$, K. Retterer ${ }^{1}$, C. Scacheri ${ }^{1}$, B. Friedman ${ }^{1}$, J. Tahiliani ${ }^{1}$, D. Copenheaver ${ }^{1}$, S. Bale ${ }^{1}$, G. Richard ${ }^{1}$, N. Smaoui ${ }^{1}$, P. Vitazka $^{1}$, J. Juusola ${ }^{1}$, E. Haverfield ${ }^{1}$

\section{GeneDx}

Whole exome sequencing (WES) became a clinical service in our CLIA lab in January, 2012; we have received samples from over 1,500 probands. Specimens were provided on the proband and at least one other biological relative in $83.5 \%$ of cases. We hypothesized that the proportion of definitive diagnoses would be higher in cases that had more than one family member submitted for WES. In an effort to test this hypothesis and to help determine the optimal number of family members to submit for WES in order to maximize the likelihood of a definitive diagnosis, we retrospectively evaluated 1,587 cases that were reported from December 2012 through May 2014 and compared the diagnostic yield amongst groups categorized by the number of family members sequenced. A molecular diagnosis was made in $29.2 \%(463 / 1,587)$ of all probands. These patients had pathogenic or likely pathogenic variants in genes consistent with the reported phenotype. For singleton cases where only the proband's specimen was submitted, a diagnosis was made in $24.5 \%$ (64/261). For duo cases where the proband and one additional family member (most often a biological parent) were sequenced, a diagnosis was made in $25.6 \%$ (23/90). A diagnosis was made in $31.0 \%(344 / 1,111)$ of trios, which most often consisted of specimens from the proband and both biological parents. The difference between the positive rate for the singletons and the positive rate for the trios was significant (Fisher's exact test, $p=0.04$ ). These results confirm our hypothesis that diagnostic yield of WES is higher when more than one family member is submitted for analysis, and suggest that trios maximize the potential for a definitive diagnosis. Based on these results, we recommend that providers submit trios for WES when feasible to maximize the probability of obtaining a definitive molecular diagnosis. 
Complexities of Genetic Counseling for Variants of Unknown Significance on Whole Exome Sequencing

M. Harr ${ }^{1}$, H. Dubbs ${ }^{1}$, E. Zackai ${ }^{1}$

\section{Children's Hospital of Philadelphia}

Whole exome sequencing (WES) is quickly becoming common practice in pediatric genetics and the genetic counselor plays a role in all aspects of the WES process including education, informed consent, scientific review of results, return of results, and formulating a follow-up plan. An important facet of the education and consent for WES is preparing families for the possibility of uncertain results including variants of unknown significance (VUS). Thousands of variants are identified in any one individual's exome, the majority of which do not necessitate further investigation. However, variants identified by the laboratory as a possible explanation for the patient's phenotype do warrant additional consideration. Genetic counseling for VUS in genetic testing is not a new challenge, however VUS identified using WES have added increased layers of complexity to the counseling session. Uncertainties involving VUS include difficulty establishing recurrence risk, limited options for future prenatal testing, confusion over multiple potential diagnoses, and uncertainty regarding medical management recommendations. Specific cases will be reviewed which highlight these issues including a VUS in a known X-linked dominant gene $(P O R C N)$ in a family with presumed $\mathrm{x}$-linked recessive inheritance; a de novo VUS in known disease gene (ANKRD11) in a patient with minimal overlap to known phenotype; compound heterozygous VUS in a novel gene (MTIF2) without a known human phenotype; and an inherited VUS in a gene ( $F B N 1)$ considered to be medically actionable. Part of the role of the genetic counselor is to guide the family in their understanding of the clinical implications of the reported VUS's and discuss options for additional research studies, re-analysis of the data, or continued investigation of the phenotype which may be necessary before the significance of any one VUS can be established. The cases we present offer specific examples of the issues surrounding genetic counseling for VUS on WES and review the strategies implemented to try and clarify the uncertainty of these findings.

Patient Perceptions of Whole Genome Sequencing Results and Non-Actionable Findings

L. Jamal ${ }^{1,2}$, J. Robinson ${ }^{2}$, M. Slashinski ${ }^{2}$, L. Feuerman ${ }^{2}$, J. Vassy ${ }^{3}$, K. Christensen ${ }^{4}$, J. Blumenthal-Barby ${ }^{2}$, J. Wycliff ${ }^{2}$, R. Green $^{4}$, A. McGuire $^{2}$

1. Johns Hopkins University

2. Baylor College of Medicine

3. General Internal Medicine, VA Boston Healthcare System

4. Department of Medicine, Brigham and Women's Hospital

Background: There remains much debate over best practices for reporting and conveying results from clinical whole exome and genome sequencing (WGS) to patients. One view holds that American College of Medical Genetics and Genomics recommendations undermine patient autonomy by restricting choice. Another contends that clinical genome analyses should be guided by professional expertise, and that clinicians have a duty to disclose life-saving information to patients. Evidence of how patients interpret and use WGS results, given the psychological complexity of decision-making, is largely absent from the debate. Methods: The MedSeq Project is a randomized trial exploring the use of WGS in cardiology and primary care. At baseline (before WGS), 106 patient-participants were surveyed and a subset of 24 completed in-person, semi-structured interviews. Transcripts of interviews were consensus coded and analyzed. Target enrollment of 200 patient-participants is expected by June of 2014, and a total of 48 participants will be qualitatively interviewed. Results: Consistent with other studies, the majority of patient-participants ( $87 \%)$ wanted all WGS results. However, qualitative data show that all interviewed patient-participants $(n=24)$ had difficulty distinguishing among result types. They struggled to provide clear examples of results associated with preventable or treatable conditions as distinct from results with less clinical utility. These difficulties did not reflect poor study understanding or genetic literacy. Most patient-participants $(n=19)$ wanted results associated with non-preventable or treatable conditions to inform their professional or financial plans, supportive care, and quality of life. Conclusion: Patients may not easily distinguish among the types of results described in WGS informed consent documents. They value WGS results for both medical and non-medical reasons. These data challenge the conceptual framework underlying many emerging models of WGS results disclosure, which define results by objective standards of clinical utility and base disclosure practices on these definitions.

\section{Genetic Testing II: Implementation}

Yield of Pathogenic/Expected Pathogenic Variants in Young Women with Breast Cancer Undergoing Hereditary Cancer Panel Testing

L. Andolina ${ }^{1}$, R. Klein ${ }^{2}$, M. Hussong ${ }^{1}$, A. Stafford ${ }^{1}$, P. Murphy ${ }^{1}$, J. Booker ${ }^{1}$, M. Cremona ${ }^{1}$, S. Solomon ${ }^{1}$, K. J. Vogel Postula ${ }^{1}$, L. Susswein ${ }^{1}$, K. Hruska ${ }^{1}$

1. GeneDx

2. BioReference Laboratories

BACKGROUND: The National Comprehensive Cancer Network currently recommends consideration of genetic testing for Hereditary Breast/Ovarian Cancer syndrome (HBOC) for women with a diagnosis of breast cancer at or before age 45, regardless of family history. Most studies investigating the frequency of mutations in $\mathrm{HBOC}$ genes have focused on $B R C A 1 / 2$. The availability of hereditary cancer panel testing via next-generation sequencing has allowed for broader testing of genes predisposing to cancer risk. However, data regarding the diagnostic yield of panels in young women with breast cancer have been sparse. Testing recommendations have not expanded beyond BRCA1/2 and a few rare cancer syndromes. We sought to determine the frequency of pathogenic/likely pathogenic variants $(\mathrm{P} / \mathrm{LPVs})$ in hereditary cancer genes in young women with breast cancer to determine the utility of panel testing. METHODS: We reviewed the cancer panel results of 552 women with invasive breast cancer or ductal carcinoma in situ, aged $<=45$ years. RESULTS: Forty nine P/LPVs were identified in 47 women $-8.5 \%$ of the study population. These were identified in ATM (6), BARD1 (2), BRCA1 (8), BRCA2 (10), BRIP1 (1), CHEK2 (12), MLH1 (1), MUTYH (2), NBN (1), PALB2 (3), PTEN (2) or TP53 (1). Two individuals were found to have 
pathogenic variants in two genes (BRCA1/MUTYH; BRCA2/NBN). In total, 31/49 P/LPVs identified were non-BRCA1/2 (63.3\%). CONCLUSION: $B R C A 1$ and $B R C A 2$ were the most commonly identified cancer genes (18/49). However, $31 \mathrm{P} / \mathrm{LPVs}$ were identified in other inherited cancer genes and would have gone undetected by testing only for $B R C A 1 / 2$. Furthermore, those patients in whom two pathogenic variants were identified would not have undergone testing for non-BRCA1/2 genes had their genetic testing been ordered as $B R C A 1 / 2$ alone or with reflex to a panel if negative. Inherited cancer panels should be considered as an initial test in the genetic diagnostic evaluation of young women with breast cancer, as the current study demonstrates a high yield of non-BRCA1/2 pathogenic/likely pathogenic variants in these patients.

\title{
Comparison of Mutation Detection in Cancer Specific versus Pan-Cancer Approaches to an At Risk Population \\ P. Kaushik ${ }^{1}$, K. Moyes ${ }^{1}$, M. Landon ${ }^{1}$, R. Wenstrup ${ }^{1}$
}

\section{Myriad Genetic Laboratories, Inc.}

Hypothesis/Purpose: Panels of genes associated with an increased risk of cancer are now clinically available. These panels fall into two categories: disease specific panels containing genes associated with one type of cancer, such as breast cancer; and pan-cancer panels containing genes regardless of the associated cancer. The purpose of this analysis is to determine the difference between cancer specific and pan-cancer panels in identification of actionable mutations. Methods: A retrospective analysis was performed on consecutive patients who tested positive for a deleterious or suspected deleterious mutation using the Myriad myRisk hereditary cancer panel between September 4, 2013 and April 17, 2014. The myRisk panel analyzes BRCA1/2, the MMR genes, APC, MUTYH, CDKN2A, CDK4, PALB2, CHEK2, SMAD4, BMPR1A, STK11, TP53, CDH1, PTEN, ATM, NBN, BARD1, $B R I P 1$, and $R A D 51 C / D$. Clinical characteristics were reviewed based on data reported on test request forms submitted to a commercial testing laboratory. Patients were then separated into those who met the National Comprehensive Cancer Network (NCCN) guidelines for BRCA1/2 testing only, those who met NCCN guidelines for Lynch syndrome testing only, those who met NCCN guidelines for both, and those who did not meet NCCN guidelines for either. Results: In this period, 9,201 patients were tested and 704 mutations were identified in 688 patients. 501 mutations were identified among patients who met the NCCN guidelines for $B R C A 1 / 2$ testing alone. Of these, unexpected findings included $4(0.8 \%)$ mutations in genes not associated with any HBOC-associated cancers (MUTYH, APC), and 20 (4\%) mutations in the MMR genes. 59 mutations were identified among patients who met the NCCN guidelines for Lynch syndrome testing alone. Of these, unexpected findings included 4 (6.8\%) mutations in genes not associated with any Lynchassociated cancers $(B A R D 1, N B N)$, and $4(6.8 \%)$ mutations in BRCA1/2. 16 patients were found to carry mutations in two different genes. Conclusions: This data shows that disease specific cancer panels may miss identifying a portion of clinically actionable mutations.

\section{Performance of Noninvasive Prenatal Aneuploidy Testing at Different Fetal Fractions}

R. McCullough ${ }^{1}$, S. Kinnings ${ }^{2}$, J. Geis ${ }^{2}$, J. Wardrop ${ }^{2}$, J. Saldivar ${ }^{2}$

1. Sequenom Center for Molecular Medicine

2. Sequenom Laboratories

Introduction: Fetal fraction is an important quality metric to evaluate when testing for chromosomal aneuploidies because the fraction of fetal DNA has a direct impact on the sensitivity of the results. Because of this relationship there have been concerns regarding the ability of noninvasive prenatal testing (NIPT) to accurately detect aneuploidies at low fetal fractions. In this study we used 211,467 clinical samples to evaluate the distribution of positivity rates at a range of fetal fractions, model predicted sensitivities, and assess potential impact on the clinical performance. Methods: An NIPT assay using massively parallel sequencing of cell-free DNA from maternal blood was performed by Sequenom Laboratories. Samples were subjected to DNA extraction and library preparation followed by massively parallel sequencing as previously described. Results: The distributions of the z-scores of 211,467 euploid and trisomy 21 samples with reportable results for NIPT were modeled and used to estimate the clinical performance at three different ranges of fetal fraction: $4-8 \%, 8-12 \%$ and $>12 \%$. This model showed that the estimated T21 sensitivity for samples with fetal DNA at 4-8 \% was $99.0 \%$ (CI 98.3-99.7\%), 8-12\% was $99.9 \%$ (CI 99.7-100.0\%), and $>12 \%$ was $99.8 \%$ (CI 99.5-100.0\%). Although the sensitivity modeling can be negatively impacted by multi-fetal and mosaic samples at higher fetal fractions, all predicted sensitivities fell within the performance demonstrated in the clinical validation. Conclusion: Fetal fraction is one of many quality metrics used in NIPT. As with any quality metric, an acceptance range must be established and validated by the laboratory to ensure consistent performance. This study demonstrates that a model based on 211,467 clinical samples exhibits an estimated performance within the validated clinical sensitivity across all fetal fractions.

\section{Diagnostic Yield of Genetic Evaluation and Testing at the Children's Hospital Colorado Autism Specialty Genetics Clinic}

\author{
B. Miller ${ }^{1}$, N. Meeks², K. Brown ${ }^{3,4}$ \\ 1. University of Colorado Denver Anschutz Medical Campus \\ 2. Department of Pediatrics, University of Colorado Denver \\ 3. Children's Hospital Colorado \\ 4. University of Colorado
}

Background: Autism spectrum disorders (ASDs) are a group of neurodevelopmental disorders characterized by varying degrees of limitations in communication and social interaction, and atypical repetitive behaviors. Several well-defined genetic disorders have been identified in patients with ASDs; however, clinical genetic testing is diagnostic in only a minority of cases. The purpose of this study was to determine the diagnostic yield of genetic testing in a population of children with ASDs seen at the Autism Specialty Genetics Clinic, part of the Autism Treatment Network, at Children's Hospital Colorado. Methods: This study was a retrospective chart review of patients who had an initial or follow-up genetics evaluation within the past 6 years, a diagnosis of an ASD through formal evaluation and at least one molecular genetic test completed and available for review ( $n=107)$. Results: The average number of molecular tests ordered per patient was 3.05 [95\% CI: 2.74-3.35]. Eighteen patients (16.82\%) had molecular genetic testing that 
was diagnostic. Chromosomal microarray (CMA) provided the highest diagnostic yield by identifying $67 \%$ of all pathogenic findings. Other findings included mutations in PTEN, MECP2 and PTPN11, positive FISH testing for a known familial mutation, and abnormalities detected on karyotype in two patients. Biochemical screening for metabolic conditions, FMRI testing, and multi-gene panels evaluating for more than one genetic condition did not yield any pathogenic findings. Of the molecular tests completed, $21.5 \%$ identified a variant of uncertain significance; an average 0.65 variants were identified per individual [95\% CI: 0.45-0.85]. Conclusion: This study provides information about the diagnostic yield of genetic testing in an ASD specialty genetics clinic, which may inform clinical decision-making and allow geneticists and genetic counselors to provide more accurate information about the potential diagnostic yield of genetic tests. Also, this study further supports the use of CMA as a first tier genetic test for the evaluation of ASDs.

The Challenge of Comprehensive and Consistent Sequence Variant Interpretation across Clinical Laboratories

\author{
M. Pepin ${ }^{1}$, S. Bailey², D. Leistritz ${ }^{1}$, M. Murray ${ }^{2}$, U. Schwarze ${ }^{2}$, P. Byers ${ }^{2}$ \\ 1. University of Washington \\ 2. Department of Pathology, University of Washington
}

Genetic testing has moved from academic laboratories that specialized in genes with which they were familiar to large laboratories that use less refined analytic tools to assess sequence variants. Are current analytic sources adequate and, if not, what should be done to improve variant interpretation? Between January 2013 and March 2014, the Collagen Diagnostic Lab (CDL) received 38 requests to interpret variants identified in outside laboratories (OL) in genes that encode connective tissue proteins [COL3A1 (21), COL1A1 (7), COL1A2 (5), TGFBR1 (1), TGFBR2 (2,) and FBN1 (2)]. Each variant was evaluated by a laboratory director and a genetic counselor. A de-identified copy of the OL report was provided in 25 instances. The interpretation and sources used for interpretation by the OL were recorded. The CDL interpretation was compared to that from the OL, discrepancies recorded, and the reasons for the discrepancies assessed. The interpretations from the OL and CDL were concordant in 12 of 38 inquiries (32\%); there were minor discrepancies in 13 instances (34\%), but significant differences in $13(34 \%)$. The discrepancies resulted from three factors: 1) known causative variants were not available in accessible databases (e.g., ClinVar/ClinGen or the Collagen Gene database (http://www.le.ac.uk/ge/collagen/) ( $n=7), 2)$ databases in which variants were recorded had not been searched $(n=7)$ and 3) knowledge of the protein structure and function was not used to interpret new variants $(n=14)$. Appropriate interpretation of sequence variants depends on an intelligent mutation database linked to clinical details and analytical tools that allow prediction of variant consequence based on gene-specific features. Laboratories that do genetic tests should require submission of appropriate clinical information with the tested sample, variants and linked clinical details should be reported to accessible databases, and laboratory certification (CAP/CLIA) should integrate compliance with data submission as a requirement for re-certification.

\title{
Performance of SNP-Based Noninvasive Prenatal Testing in Low-Risk Women
}

\section{Stosic ${ }^{1}$, K. Curnow ${ }^{1}$, M. P. Hall ${ }^{1}$, B. Zimmerman ${ }^{1}$, M. Hill ${ }^{1}$, Z. Demko ${ }^{1}$, M. Rabinowitz ${ }^{1}$}

\section{Natera, Inc.}

Introduction: There is growing discussion regarding the utility of noninvasive prenatal testing (NIPT) in low risk populations. Purpose: To determine the positive predictive value (PPV) of a high-risk result for fetal aneuploidy with single-nucleotide polypmorphism (SNP)-based NIPT in low-risk women. Methods: NIPT performance was evaluated in 12,104 low-risk women: 474 low-risk women from a clinical trial (Cohort A), and 11,630 low-risk women among a general screening population that presented for NIPT (Cohort B). Patients in Cohort A were determined to be low-risk based on serum screening results, a lack of ultrasound abnormalities, and a maternal age of less than 35. For Cohort B, women were defined as low-risk if maternal age was under 35; no other criteria for determining risk were available. Maternal blood samples were analyzed at Natera, Inc., a CLIA-certified laboratory in San Carlos, CA. Isolated cfDNA was amplified via multiplex PCR targeting 19,488 SNPs covering chromosomes 13, 18, 21, X, and Y. Sequencing data was analyzed using the NATUS algorithm and reports were issued describing risk for trisomy 21, trisomy 18, trisomy 13, and Monosomy X. Karyotype information was available for all patients in Cohort A. For Cohort B, clinical outcomes were requested from providers on cases receiving a high-risk result. PPV [(true positives)/(true positives + false positives)] was calculated for cases with known karyotype. Results: Of the 474 cases in Cohort A, 5 $(1.0 \%)$ cases received a high-risk result: all calls were correct. Of the 11,630 cases in Cohort B, $118(1.0 \%)$ cases received a high-risk result. In Cohort B, karyotype was available for 16 cases with high-risk results, revealing 13 true positives and 3 false positives; the majority of high-risk calls without karyotype were patients that declined invasive testing. For the four aneuploidy indications combined, the PPV for Cohorts A and B were 100 and $81.3 \%$, respectively. Conclusion: The high PPV in both low-risk cohorts supports that this SNP-based NIPT method offers a highly-accurate screening approach for all women, independent of prior risk

\section{Pediatrics}

Chinese Parents' Perception of Autism Spectrum Disorders: An Exploration of the Influence of Culture

J. Chen ${ }^{1}$, L. Lichten ${ }^{2}$, P. Delerme ${ }^{3}$, B. Sheidley ${ }^{1,4}$

1. Genetic Counseling Program, Brandeis University

2. Department of Pediatrics, Boston University School of Medicine

3. Department of Maternal-Fetal Medicine, Obstetrics and Gynecology, Tufts Medical Center

4. Department of Neurology, Boston Children's Hospital

Autism spectrum disorder (ASD) is a neurodevelopmental disorder characterized by impaired communication skills, repetitive behaviors, and restricted interests. Numerous studies have examined the underlying causes of ASD and have identified causative genetic and environmental factors. Researchers have also explored parental perceptions regarding the etiology of ASD. These studies have demonstrated that parents endorse a wide variety of causes for 
ASD, including genetic factors, vaccinations, prenatal exposures, and other environmental factors. However, there are few studies addressing parental perceptions among minority or immigrant populations, who may be likely to hold beliefs that are heavily influenced by their own culture. The purpose of this study was to explore the impact of Chinese culture on perceptions of causality, treatment, and family dynamics among parents of children with ASD. We conducted semi-structured interviews with six Chinese-American mothers of at least one child with ASD that were recruited through Boston Chinatown Neighborhood Center. We analyzed the interview transcripts for common themes using ATLAS.ti software. Overall, we found the experiences and beliefs of our study participants were similar to those of non-immigrant parents described in previous studies. Our study participants believed in Western notions of causality (including genetics) and treatment (such as ABA therapy), and experienced guilt, shame, and blame in their families and social networks after the diagnosis. However, our study participants also had some experiences that were unique to their status as immigrants, such as additional grief over the loss of opportunity in the United States for their child and further isolation caused by language barriers and geographical distance from extended family members. Our results suggest a need for additional support for Chinese immigrant parents who have a child with ASD and highlight the need for further research about the experiences of other minority populations after receiving a diagnosis of ASD.

\title{
Left out in the Cold: Barriers to Clinical Trial Participation and the Impact on Parents of Sons with Duchenne Muscular Dystrophy
}

\author{
L. Murray ${ }^{1}$, B. Denger ${ }^{2}$, L. Mandel ${ }^{1,3}$, H. Peay ${ }^{2}$, E. Estrella $^{4}$ \\ 1. Brandeis University \\ 2. Parent Project Muscular Dystrophy \\ 3. Regis College \\ 4. Department of Genetics and Genomics, Boston Children's Hospital
}

The primary goal of clinical trials is to advance understanding of human disease, yet participants may directly benefit through involvement in such trials. Novel therapeutic clinical trials specific to Duchenne muscular dystrophy (DMD), a life-limiting condition, have begun to show promising results, but inclusion criteria for these trials often require ambulation, a criterion that most older boys with DMD do not meet. The purpose of this study was to explore the emotional impact on parents of boys with DMD who do not meet inclusion criteria for novel therapeutic clinical trials due to loss of ambulation. We mailed recruitment notices to families of boys with DMD who were non-ambulatory and who had participated in the Harvard Neuromuscular Disease Project. We interviewed 6 mothers and one father of 7 unrelated boys with DMD using a semi-structured interview guide to assess the impact of exclusion from clinical trials on parental hopefulness, expectations for the future, and continued interest in clinical research. We used Atlas.ti software to identify and code themes that emerged from the interview transcripts. Four themes emerged: (1) Ambulation represents a barrier to research participation, and parents are often frustrated by exclusion. (2) Parents conveyed resignation to their sons' condition, ineligibility for research, and the limited potential for effective, novel therapies. (3) Parents' active interest in clinical research opportunities waned. (4) Parents still expressed hope for their sons, often related to the concept of emotional well being, but also to the possibility of a cure or slowing the progression of DMD. These results highlight how parents rationalize being excluded from clinical trials, but still maintain a hopeful attitude overall. Our findings, suggest that genetic counselors and health care providers need to be mindful of the emotional impact of exclusion and continue addressing issues surrounding this topic.

\section{Family History of Epilepsy and Central Nervous System Comorbidities as a Prognostic Indicator for Resective Surgery Outcomes among Pediatric Epilepsy Patients}

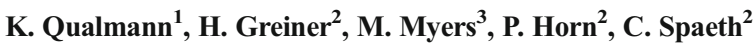 \\ 1. University of Cincinnati \\ 2. Cincinnati Children's Hospital Medical Center \\ 3. Genetic Counseling Graduate Program, University of Cincinnati
}

Patients with epilepsy have higher rates of certain central nervous system (CNS) comorbidities over the general population. The presence of some comorbidities in probands has been linked to the persistence of seizures following resective brain surgery. While a family history (FHx) of seizures has been associated with comorbidities in probands, no studies have investigated the rates of comorbidities in other relatives or how they may influence probands' resective surgery outcomes. We hypothesized that key FHx risk factors influence prognosis for seizure free outcome following resective surgery. Parents of children with epilepsy, ages $0-17$, who received resective surgery at Cincinnati Children's Hospital Medical Center (CCHMC) were invited to participate in the study. A three-generation pedigree of epilepsy and its CNS comorbidities was collected via an online or phone survey from 52 participants. Surgery outcome classification by the International League Against Epilepsy scale was abstracted from an existing clinical database at CCHMC. First-degree relatives (FDRs) had significantly higher rates of ADHD $(p<0.001)$, autism $(p=0.020)$, depression $(p=0.002)$, and motor disability $(p<0.001)$ over the general population. Total relatives identified in the study had significantly higher rates of depression $(p<0.001)$, motor disability $(p=0.005)$, and epilepsy/seizures $(p<0.001)$. Poorer surgery outcomes were significantly associated with the presence of autism in FDRs $(p=$ $0.019)$ and total relatives $(p=0.037)$, with the odds of a poor outcome increasing by a factor of 2.6 for every $10 \%$ increase in proportion of affected FDRs $(95 \% \mathrm{CI}=1.0,6.9 ; p=0.045)$. These data provide novel insights into the importance of FHx and the role of genetic counselors in the assessment of a child with epilepsy. The increased rates of comorbidities in other relatives support the hypothesis of a shared heritability and pathophysiological mechanism with epilepsy. Furthermore, the association between a FHx of autism and worse surgery outcomes indicates a role for FHx information in probands' prognostic assessment prior to undergoing resective surgery.

Parents Report Impaired Health-Related Quality of Life in Children with Methylmalonic Acidemia with Improvements in Development and Health following Liver Transplantation

K. Splinter ${ }^{1}$, A. K. Niemi ${ }^{1}$, R. Cox ${ }^{1}$, A. Kwan ${ }^{1}$, J. Platt ${ }^{1}$, M. Shah ${ }^{2}$, G. Enns ${ }^{1}$, M. Kasahara ${ }^{3}$, J. Bernstein ${ }^{1}$

1. Stanford University 
2. University of California San Francisco

3. National Center for Child Health and Development

Introduction. Methylmalonic acidemia (MMA) mut0 subtype is a metabolic disorder with risk for neurologic damage during periods of metabolic stress. To prevent such outcomes, liver transplantation (LT) has emerged as a treatment option. However, complications of MMA still occur post-LT. Although the natural history of MMA is well documented, the psychosocial impacts of MMA, including MMA post LT, on children and families are not well defined. Purpose. The goal of this study was to measure health related quality of life (HRQoL) of children and families affected by MMA and to assess the impacts of LT in MMA on HRQoL. Methods. Parents of children with MMA were recruited through the Organic Acidemia Association, Stanford Children's Hospital, and other genetics providers. A survey was used to gather clinical information and assess HRQoL using the PedsQLTM Generic Core Scales, Transplant Module, and Family Impact Module. Mean HRQoL scores of LT and non-LT samples were compared using independent t-tests. Results. Thirty-five responses regarding children (mean age: 8.0 year (SD: 4.9)) with MMA mut0 were received; 16 of these responses (46 \%) were regarding children (mean age: 8.4 years (SD: 4.5)) who had undergone LT. Results showed the lowest HRQoL scores for children in school functioning and social interactions. No significant differences in HRQoL were found between the LT and non-LT samples on many of the scales. $90 \%$ of parents expressed LT had a positive impact on their child's development, citing improvements in health and increased ability to participate in social activities. Conclusions. Our results revealed HRQoL in children with MMA is impaired with lowest scores in school functioning and social domains. Our results also demonstrate that parents feel LT positively impacts development and health of children with MMA and may improve social interactions.

\title{
The Relationship between Birth Weight and the Variability of Medical Complications in Patients with Cystic Fibrosis
}

\author{
R. Nelson ${ }^{1}$, R. Darrah ${ }^{1}$, A. Matthews ${ }^{1}$, C. Hodges ${ }^{1}$ \\ 1. Case Western Reserve University
}

One complication of cystic fibrosis (CF) is an inability to gain and maintain weight. Previous studies have identified a positive correlation between body weight and pulmonary function of young patients. Consequently, growth failure can be a useful indicator of the morbidity and mortality of CF. As growth failure in patients with CF may be observed as early as birth, this study aimed to characterize birth weight in patients with CF and identify the predictive quality of birth weight on later medical complications. Patients cared for at the CF Center at Rainbow Babies and Children's Hospital participated in this study $(n=79)$. Independent samples t-tests identified that males with CF $(n=40)$ were on average $10.24 \mathrm{oz}$ lighter at birth than the national male average $(p<0.01)$ and females with CF $(n=39)$ were on average 9.04 oz lighter at birth than the national female average $(p=0.01)$. Linear regression analyses showed birth weight was positively correlated to percentage of forced expiratory volume (FEV1\%) at age $6(n=54, \mathrm{R} 2=0.08, p=0.04)$ and at age $10(n=$ $59, \mathrm{R} 2=0.07, p=0.05$ ) but there was no correlation at ages 15 and 20. Birth weight was not predictive of the age at Pseudomonas colonization or the incidence of cystic fibrosis related diabetes (CFRD). These findings suggest that birth weight can predict pulmonary function at young ages; however, the lack of correlation between birth weight and later pulmonary function, the age of Pseudomonas colonization, or development CFRD suggests that other variables influence these later phenotypes. These data suggest that growth deficiency in CF begins prenatally and that birth weight could be used as the first phenotypic measure to predict pulmonary function. These data may allow for early identification of patients with CF at risk for lower pulmonary function in childhood and assist genetic counselors in providing anticipatory guidance to patients and families.

A Follow-Up Study to the Possible Role of Pediatric Genetic Counselors in the International Adoption Process of Children with Special Needs E. White ${ }^{1}$, L. Seaver ${ }^{2}$

1. Wentworth-Douglass Hospital

2. Kapiolani Medical Specialists

A successful pilot study was previously presented as a poster abstract at the 2012 NSGC Annual Education Conference utilizing a board certified genetic counselor and geneticist in providing informal genetic assessments of 28 children with special needs in China placed for international adoption. The study suggested that genetic counselors and geneticists may be able to play a role in pre-adoption record review that will assist potential parents in decision making and planning related to international adoption of special needs children. Subsequently, an 11 question survey was conducted of medical geneticists, PhD geneticists, and genetic fellows attending the 2012 David W. Smith Workshop to explore their interests and experiences with providing genetic assessments of international adoptees. There were 85 survey respondents among $136(62.5 \%)$ workshop attendees. Seventy-five providers $(88 \%$ of respondents) felt that genetic assessments on such children would be somewhat or very helpful in decision-making to pre-adoptive parents. When asked to predict how this information will affect inter-country adoption rates, twenty-four respondents (28\%) thought it would somewhat or greatly increase the adoption rate; twenty-seven (32\%) felt it would not increase the adoption rate; and 26 individuals (30\%) predicted this information would decrease the adoption rate. Fifty-nine individuals $(69 \%)$ indicated that they would be interested in providing long distance advice regarding potential diagnoses of international adoptees with special needs. Insights, stories or comments were provided by 30 respondents. Themes included affirmation of the poor quality of medical abstracts on previously assessed international adoptees and the importance of determining the motive of pre-adoptive parents. Future studies on the benefits of assessment for parental decision making and planning are warranted.

\section{Prenatal}

Evaluation of the Quality and Literacy of Commercial Noninvasive Prenatal Test Websites

M. Bell ${ }^{1,2}$, B. Biesecker ${ }^{3}$, D. Roter ${ }^{2}$

1. National Human Genome Research Institute

2. Johns Hopkins Bloomberg School of Public Health 


\title{
3. Genetic Counseling Training Program, Johns Hopkins University/National Human Genome Research Institute
}

The introduction of noninvasive prenatal testing (NIPT) in 2010 has presented challenges in clearly, accurately, and effectively explaining the benefits and limitations of this test in the media, especially on commercial websites of companies providing NIPT. In light of these challenges, this study examines the quality and readability of four primary commercial NIPT websites created for new and expectant parents and proposes recommendations for improving the utility of these resources. Using the validated DISCERN-Genetics tool, the websites were evaluated for content quality and effectiveness in facilitating decision-making. Content readability was assessed by the Flesch-Kincaid reading level score. The DISCERN-Genetics tool scored each site on criteria based on five content areas: aims of the publication, background of the condition of interest, the genetic test and results, implications of the test, and reliability of information. Overall, the DISCERN quality scores of the websites ranged from $68 \%$ (fair) to $82 \%$ (good). We found that all four sites lacked explicitly stated aims and explanations of the accuracy and positive predictive values of the test. Additionally, one site lacked explanations and supplementary links to more information about the fetal chromosome abnormalities tested for by NIPT. Furthermore, all of the websites presented material at a reading level above the 10th grade, which exceeds the recommended 8th grade level. In order for expectant parents to better utilize these sites and make decisions about the use of NIPT for their pregnancy, sites should: clearly state the aims of the website, display sensitivity and specificity data in an accessible manner, include trustworthy information and links to resources about fetal chromosomal abnormalities, and reduce the literacy demands of the content. In conclusion, greater awareness of the quality and readability of genetic testing websites is needed, especially as new genetic tests become available.

\section{Continuing a Pregnancy following Prenatal Diagnosis of a Lethal Fetal Defect is Associated with Improved Psychological Outcome}

\author{
H. Cope ${ }^{1}$, M. Garrett ${ }^{1}$, S. Gregory ${ }^{1}$, A. Ashley-Koch ${ }^{1}$
}

\section{Duke University Medical Center}

Previous research indicates that pregnancy loss due to fetal anomalies commonly results in psychiatric symptoms including grief, post-traumatic stress and depression. Prior studies primarily focused on women who terminated pregnancies and included heterogeneous study groups with mild to severe fetal anomalies. However, lethal fetal diagnoses are distinct from those compatible with survival as health care providers are more likely to be directive regarding pregnancy management. A paucity of research exists on the psychological impact of continuing a pregnancy following a lethal fetal diagnosis. This project investigated a diagnostically homogeneous group comprised of 158 women and 109 men who lost a pregnancy to anencephaly, a uniformly lethal neural tube defect. Participants completed the Perinatal Grief Scale, Impact of Event Scale-Revised and Beck Depression Inventory-II, which measure symptoms of grief, post-traumatic stress, and depression, respectively. Demographics and pregnancy management (continued vs. terminated) were also collected. Gender-specific Analysis of Variance (ANOVA) was performed for instrument total scores and subscales. Time since pregnancy loss was used as a covariate in all models. Slightly more participants continued the pregnancy than terminated (58\% of women; $55 \%$ of men). Women who terminated reported significantly more grief $(p=0.01)$, active grief $(p=0.007)$, despair $(p=0.001)$, avoidance $(p=0.002)$ and depression $(p=0.03)$ than women who continued the pregnancy. Among participants who terminated, men whose partner terminated in the second trimester reported significantly more active grief $(p=0.045)$, post-traumatic stress $(p=0.028)$, intrusions $(p=0.002)$ and depression $(p=0.038)$ than men whose partner terminated in the first trimester. Our data suggest that different factors impact psychological outcome in women and men. Following a lethal fetal diagnosis, the risks and benefits, including psychological effects, of termination and continuation of pregnancy should be discussed in detail with an effort to be as nondirective as possible.

\section{Clinical Experience of Trisomy 16 and 22, and Microdeletion Detection by Noninvasive Prenatal Testing}

N. Dharajiya1 ${ }^{1}$, T. Monroe ${ }^{2}$, D. Farkas², T. Boomer ${ }^{2}$, J. Wardrop ${ }^{2}$, J. Jesiolowski ${ }^{2}$, E. Almasri², C. Zhao ${ }^{2}$, C. Deciu ${ }^{9}$, J. S. Saldivar ${ }^{2}$

\section{Sequenom Center for Molecular Medicine \\ 2. Sequenom Laboratories}

Introduction: Noninvasive prenatal testing (NIPT) has become part of the standard of care in high-risk pregnancies due to high sensitivity and specificity for autosomal aneuploidies. Building on the success and knowledge of analyzing circulating cell free DNA for trisomy 21,18 and 13 in $>250,000$ cases, we expanded the technology used in our laboratory developed test (LDT) to investigate subchromosomal deletion and duplication events. A novel algorithm to identify fetal microdeletion and microduplication events in maternal plasma has been developed by Sequenom Laboratories. We used this approach to identify microdeletions 5pdel, 22q11del, 15qdel and 1p36del, as well as trisomies 16 and 22. Methods: Maternal blood samples submitted to Sequenom Laboratories for MaterniT21TM PLUS testing were subjected to DNA extraction, library preparation, and whole genome massively parallel sequencing as described by Jensen et al. Sequencing data were analyzed using a novel algorithm to detect trisomies and other microdeletions. Results: In testing more than 75,000 samples, the MaterniT21TM PLUS test identified 23 cases of trisomy 16, 20 cases of trisomy 22, 16 cases of 22q11 deletion syndrome, 5 cases of 15qdel associated with Prader Willi/Angelman syndrome, 2 cases of 1 p36 deletion and 3 cases of 5p deletion associated with Cridu-Chat syndrome. The overall positive predictive value for each chromosomal aberration ranged from $33 \%$ to nearly $100 \%$. The total false positive rate was $0.01 \%$ for confirmed false positives and $0.02 \%$ for false positive plus non-confirmed results with no clinical evidence supporting our finding. Conclusion: Initially used for detecting trisomy 21 only, MaterniT21TM PLUS has advanced into a LDT for also detecting trisomy 18, trisomy 13, and sex chromosome aneuploidies. Here, we demonstrate continued expansion of the technological capabilities to effectively detect microdeletions and two additional rare trisomies, with a very low false positive rate.

\section{Current Practices in Prenatal Genetic Counseling regarding a Diagnosis of Down Syndrome}

E. Moe ${ }^{1}$, M. Marvin ${ }^{1}$, K. Sheets ${ }^{2}$, C. Couyoumjian ${ }^{3}$, E. Higuchi ${ }^{4}$, B. Yashar $^{5}$ 
3. University of Michigan Health System

4. University of Wisconsin-Madison

Introduction: The 2011 NSGC "Practice Guidelines for Communicating a Prenatal or Postnatal Diagnosis of Down Syndrome" provide a framework for discussions between genetic counselors and patients; however, adherence is unknown. This study explored self-reported adherence and factors affecting discussion of the guideline's "Essential Information" (EI). We hypothesized that the greatest variation in adherence would center on discussions related to the lived experience (LE) of the diagnosis. Methods: A 48-item survey created by an expert panel measured EI concept discussion frequency (Likert scored 1[never]-5[always]) and the influence of reasons for non-discussion. Prenatal genetic counselors were recruited through NSGC/American Board of Genetic Counselors listservs $(n=271)$. A combination of principal component analysis, t-tests, correlations, and ANOVA measured the impact of various patient and participant characteristics on EI discussion frequency. Results: Respondent demographics closely mirrored the 2012 Professional Status Survey. All EI concepts were discussed most of the time (Likert scores $=3.7-5.0$ ). The greatest variability was seen in the frequency of discussing LE concepts (PCA component $=27 \%$ of variance). Counseling length $>1 \mathrm{~h}(p=0.001)$, increased clinical experience $(p=0.011)$, and reading the guidelines $(p=0.027)$ were associated with a higher frequency of discussing LE concepts. Patient-oriented factors (stated intention for pregnancy management and emotional state) were more influential than counselor-oriented factors (comfort with or perceived value of the information) for not discussing certain LE concepts. Conclusions: Genetic counselors self-report a high level of adherence to the guidelines, with most variation related to discussion of the LE. When genetic counselors do not discuss all EI, they are typically tailoring their discussions based on the perceived and stated needs of the patient. Defining the appropriate balance of client-centered counseling and guideline adherence requires consideration of both clinician decisionmaking and patient expectations/satisfaction.

\title{
Termination of Pregnancy for Aneuploidy: Are Things Changing?
}

K. Morris ${ }^{1}$, H. Nelson ${ }^{1}$, C. Rebecca ${ }^{2}$, W. DiNonno ${ }^{3}$, S. Harris ${ }^{4}$, C. M. Osborne ${ }^{4}$, A. Hawkins ${ }^{5}$, V. Mathur ${ }^{6}$, K. Siefkas ${ }^{7}$, B. Stevens ${ }^{8}$, M. Tucker ${ }^{9}$, S. Weiss ${ }^{10}$, K. Ziegler ${ }^{11}$

1. University of New Mexico

2. The University of Texas Health Science Center at Houston

3. Eastern Virginia Medical School

4. University of North Carolina at Chapel Hill

5. Stanford University

6. Ben Taub General Hospital

7. Yakima Valley Memorial Hospital

8. Baylor College of Medicine

9. St. Vincent Women's Hospital

10. University of Washington

11. Ohio State University

Purpose: To evaluate current rates of termination of pregnancy due to aneuploidy, and to evaluate the influence of geographic location and other factors on termination decisions. Materials and methods: Investigators were recruited from the NSGC Prenatal Special Interest Group. Investigators reviewed medical records of prenatal diagnoses from 2007 to 2012. Inclusion criteria were: singleton pregnancy, diagnosis of non-mosaic aneuploidy, diagnosis by 24 weeks' gestation, known pregnancy outcome, maternal age 18 or older. Type of procedure, reason(s) for testing, and presence of ultrasound anomalies (malformations and soft markers) were noted. Maternal age, race/ethnicity, religion, and type of insurance were recorded. Data were submitted to KLM online via REDCap survey. Statistical analyses were completed by a statistician at University of New Mexico. Results: Data were collected on 972 pregnancies from 11 study centers across the United States. Overall termination rate was $62.1 \%$. There were 502 pregnancies with Down syndrome, of which 333 were terminated (66.3\%). Termination rates for other aneuploidies were as follows: trisomy 13: 59 \%, trisomy 18 66 \%, 45,X 43.3\%, 47XXY, $47.4 \%$. There were significant correlations $(p<0.001)$ between the decision to terminate and each of the following: type of diagnostic procedure $(C V S>$ amnio), study center location (California $>$ others, midwestern Catholic hospital $<$ others), ethnicity (Asian $>$ others), type of insurance (private $>$ Medicaid), maternal age (older $>$ younger) and ultrasound abnormalities (none $>$ anomalies noted). Conclusions: Our data suggest that fewer women overall are choosing to terminate pregnancies with a diagnosis of fetal aneuploidy now than in the past ( $p<.0001$ compared to a large 2006 study). These results also suggest that maternal ethnicity and geographic location influence decisions regarding termination. Our study confirms previously published trends: that earlier diagnosis, older maternal age, absence of ultrasound abnormalities, and more severe diagnosis predict a decision to terminate

\author{
Microarray Results \\ K. Stumm ${ }^{1}$, K. Kellom², L. Conway ${ }^{3}$, B. Bernhardt ${ }^{2}$ \\ 1. Paoli Hospital Cancer Center \\ 2. University of Pennsylvania \\ 3. Genetic Counseling Program, Arcadia University
}

"I Kind of Just Went along with It." An Exploration of the Experiences and Needs of Partners of Women Receiving Uncertain Prenatal

Chromosomal microarray (CMA) analysis detects more chromosomal abnormalities than traditional karyotyping, making CMA analysis likely to become a first-tier test for prenatal diagnosis. Previous research on women receiving positive or uncertain prenatal CMA results has documented that such results cause distress and complicate reproductive decision-making. Little research has been done on the experience of men whose partners receive uncertain results from prenatal testing. Accordingly, we sought to explore how partners of pregnant women understand and make meaning of uncertain CMA results, and how CMA analysis may impact subsequent decision-making. We conducted semi-structured telephone interviews with male partners of eight pregnant women after receiving an uncertain CMA result. Audiofiles from the interviews were transcribed, coded, and analyzed using a 
grounded theory approach. Themes from the interviews were differentiated into five domains: decision making factors, desire for and impact of information, roles of men in pregnancy, emotional experiences, and suggestions for improving the CMA testing process. Participants employed 'rational thinking' as a means to make pregnancy decisions. All of the participants were motivated to have CMA testing to gain information about their baby's health without carefully weighing the risks, benefits, and limitations of testing. When faced with results of uncertain significance, some of the men struggled to remain rational and analytical in the face of powerful emotions. Our participants described various, conflicting roles in pregnancy decisionmaking, including: supporter/protector, collaborator, or hands off — 'along for the ride.' This study has implications for understanding the impact of prenatal CMA testing and uncertain CMA results on partner's decision-making in pregnancy. Based on our results, we suggest: the need for a robust informed consent process; careful pre- and post-test counseling for prenatal CMA testing; and that professionals acknowledge the partner's needs and find ways to engage men in prenatal care.

\section{Posters}

\section{Access and Service Delivery}

Access to Genetic Counseling and BRCA Testing Among a Population-Based Sample of Black Women with Early-Onset Breast Cancer

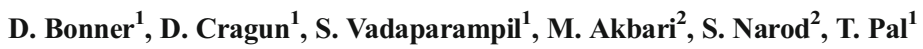

1. Moffitt Cancer Center

2. Women's College Hospital Research Institute

Purpose: Concerns about the potential for genomic advances to increase health disparities have been raised. Within the United States (U.S.), although any physician may order BRCA testing, conduct of genetic counseling (GC) prior to BRCA genetic testing (GT) remains the gold standard, endorsed by many professional organizations. Thus it is important to assess referral and uptake of GC and access to GT in minority populations. Methods: Black women diagnosed with invasive breast cancer $(\mathrm{BC})<$ age 50 in 2009-2012 were recruited through the Florida State Cancer Registry 6-18 months following diagnosis and completed a baseline questionnaire. Summary statistics and logistic regression were used to examine associations between demographic variables and access to GC and GT at enrollment. Results: All 356 participants met national criteria for referral for GC, yet only 121 (34 \%) were referred, of which 72 attended (51 of whom underwent GT). An additional 68 underwent GT (including 20 referred for GC who did not attend). Additionally, there were $181(51 \%)$ who were never offered GC or BRCA testing. The only variable associated with having GC was referral by a healthcare provider $(p<.001)$. Variables positively associated with receiving GT included GC, private health insurance, younger age, and higher household income (all $p<.01$ ). Marital status and having had children were not associated with undergoing GT. Conclusions: The population-based study design provides a more accurate estimate of access compared to other designs and results suggest efforts are needed to improve access to GC and GT. Our results indicate that the majority of participants were not referred to or attended GC. Furthermore, referrals by healthcare providers were a key determinant in GC access. Even among an ethnic minority population, disparities in access to GT exist and they appear to be due, at least in part, to socioeconomic factors and referral patterns of physicians.

\section{Clinical Practices of Neurologists Related to Predictive Testing of Presymptomatic Patients at Risk for Huntington's Disease}

\section{Bradley ${ }^{1,2}$, F. Revilla ${ }^{1}$, H. He ${ }^{2}$, T. Burrow ${ }^{2}$, S. Hufnagel ${ }^{2}$, E. Mundt ${ }^{2}$, K. Collins ${ }^{2}$}

1. University of Cincinnati

2. Cincinnati Children's Hospital Medical Center

Huntington's disease (HD) is a neurodegenerative condition that results in a progressive movement disorder accompanied by psychiatric alterations and cognitive impairment. The availability of a predictive genetic test for HD raised moral and ethical concerns in the health care community, prompting organizations to establish testing guidelines. The primary aim of this study was to determine if neurologists offer predictive testing to presymptomatic patients at risk for HD. The secondary aims were to determine the clinical practices neurologists follow during the predictive testing process (pre-test discussion, pre-test practices, post-test practices), assess the frequency with which these practices occur and obtain neurologist's thoughts on the predictive testing guidelines published by the European Huntington Disease Network (EHDN) in 2012. The study included a cross-sectional survey of neurologists from the International Parkinson and Movement Disorder Society who are currently practicing in the United States $(n=1,249)$. The survey questions were based on recommendations from the $2012 \mathrm{EHDN}$ guidelines. One hundred thirty one surveys met inclusion criteria for this study (response rate of $10.5 \%$ ). Two thirds of neurologists $(64.1 \%)$ report that they have experience ordering predictive testing for HD. The frequencies of their clinical practices were assessed. Physicians frequently discussed the autosomal dominant nature of the HD gene (98.8 \%) and the possible impact on the patient's family $(94.1 \%)$. They did not frequently contact a patient with a negative test result within 1 week of results disclosure (25.6\%). Thirty-four percent of neurologists who report ordering predictive testing for $\mathrm{HD}$ also report that they were familiar with the 2012 EHDN guidelines. There were no significant differences in the clinical practices of neurologists who reported following the guidelines compared to those unaware of them or those who choose not to follow them. Not all neurologists familiar with the guidelines follow them completely.

A Survey of Genetic Counselors regarding the Impact of Recent State Legislation Restricting Access to Abortion on Patients and Practice

C. Cooney ${ }^{1}$, L. Hercher ${ }^{1}$

1. Sarah Lawrence College 
In 2013, 22 states moved to restrict access to abortion, enacting a total of 70 provisions. Examples of legislation include: increases in regulation targeting abortion providers and facilities, limits on procedures tied to gestational age (some have been rejected by the courts as unconstitutional), restrictions on funding sources available to women seeking abortions, and obstacles intended to discourage abortion such as mandatory waiting periods and mandatory ultrasounds. We hypothesized that genetic counselors working in regions where legislation restricting abortion had been enacted would be more likely to report an effect on options available for their patients. A survey of 37 questions was developed to gauge genetic counselors' professional opinion on the impact of provisions restricting abortion on colleagues, patients, prenatal genetic counseling sessions, hospital policies, and screening recommendations. A total of 286 individuals completed portions of the survey; not all respondents answered all questions. Results were stratified by regions based on United States Census Bureau designations (Northeast, South, Midwest, \& West). Genetic counselors in all regions share the same professional opinion on the provisions. (On 25 questions requesting counselors' opinion on provisions affecting abortion, all Likert scale responses for each region were within 1 standard deviation from the total mean responses). More genetic counselors in the South and Midwest reported recent changes affecting patient care. ( $28.3 \%$ of the counselors in the South and $10.6 \%$ in the Midwest noticed changes in their counseling sessions compared to $2.1 \%$ of the counselors in the Northeast and $5.5 \%$ in the West). Half of counselors in these regions experienced restrictions in patient access to second trimester abortions (52.2\%). The regional differences correlate with the location of states that have seen the greatest increase in legislative activity creating restrictions on access to abortion, suggesting that new laws are impacting genetic counselors' ability to deliver optimal patient care.

\section{The Impact of Association for Molecular Pathology v. Myriad Genetics Inc. on Cancer Genetic Counseling Practice}

\section{Costello ${ }^{1}$, L. Hercher ${ }^{1}$}

\section{Sarah Lawrence College}

The U.S. Supreme Court decision in Association for Molecular Pathology (AMP), et al. v. Myriad Genetics Inc., et al. has significantly altered the landscape of genetic testing for $B R C A 1 / 2$ specifically, and genetic predisposition testing for breast cancer more generally. Since this decision, multiple competing laboratories have begun offering diagnostic testing for mutations in BRCA1/2. Many laboratories have also introduced cancer panels, or added $B R C A 1 / 2$ to existing multi-gene tests. The current study was undertaken to explore the immediate impact of $A M P v$. Myriad on genetic counseling practice. Genetic counselors were hypothesized to be willing to change their ordering practices to reflect competitive pricing despite the advantages related to Myriad's database of $B R C A 1 / 2$ variants, and resultant curatorial edge. Additionally, it was hypothesized that the inclusion of $B R C A 1 / 2$ testing would increase the use of cancer panels as a first step in testing, despite concern about variants of uncertain significance. To date, there is no published research regarding the impact of $A M P v$. Myriad on genetic counseling. To this end, a 44 question survey was developed to gather information regarding cancer genetic counselors' practices and opinions after AMP v. Myriad. The results of this study indicate that $A M P$ v. Myriad had an immediate impact on genetic counseling practice. $80.7 \%$ of genetic counselors surveyed now use a variety of providers for $B R C A 1 / 2$ testing. Counselors indicated that their ordering practices for breast cancer predisposition testing were highly variable. $75.5 \%$ of respondents order expanded panel testing for $\mathrm{HBOC}$ as a first line test rather than $B R C A 1 / 2$ testing some of the time. Strategy depends on pedigree primarily $(95.1 \%)$ but also concern about insurance (59.8 \%) and patient wishes (59\%). $69.0 \%$ of respondents order $B R C A 1 / 2$ testing and reflex to expanded panel testing for HBOC some of the time. Strategy depends primarily on pedigree $(92.5 \%$ ) but also concern about insurance $(31.8 \%)$ and patient wishes $(60.8 \%)$. Results of the study suggest the need for evidencebased practice guidelines for cancer genetic counseling.

\section{A Descriptive Study of Current Hereditary Breast Cancer Knowledge and Clinical Practices among Florida Providers \\ D. Cragun ${ }^{1}$, L. Camperlengo ${ }^{1}$, E. Robinson ${ }^{1}$, S. Vadaparampil ${ }^{1}$, T. Pal ${ }^{1}$}

\section{Moffitt Cancer Center}

Purpose: Recent events have changed the landscape in genetic services delivery for Hereditary Breast and Ovarian Cancer (HBOC). Our objective was to describe current practitioner knowledge and practices. Methods: Florida providers $(N=539)$ who order testing for HBOC were mailed a survey in November 2013 and non-respondents were mailed a second copy in January 2014. Descriptive statistics characterized participant responses. Results: Participants completing the survey $(N=107)$ included: 7 genetic counselors; 3 medical geneticists; 3 nurses; 27 nurse practitioners; and 67 physicians; with the majority $(n=74)$ indicating either no genetics education or Myriad provider education as their sole source of genetics training. Although $80 \%$ were aware of the Supreme Court ruling that led to the loss of the BRCA patent previously held by Myriad, $83 \%$ never ordered a BRCA test from a different laboratory due primarily to a lack of perceived advantages $(n=48)$ and/or lack of awareness that other labs offer BRCA testing $(n=37)$. Despite the limited time reportedly spent on pre-test counseling (median $=21 \mathrm{~min}$ ), $>80 \%$ of respondents indicated that they usually cover 13 of 18 nationally recommended counseling elements. Elements not routinely covered by $>40 \%$ of respondents included: 1) the possibility of a variant of uncertain significance (VUS); and 2) issues related to life/disability insurance. Other areas of concern based on case-scenarios included: 1) $>70 \%$ recommending breast ultrasound for routine screening in a young female BRCA carrier; 2) $88 \%$ failing to recognize that no genetic testing would be clinically indicated in an unaffected sister of a breast cancer patient identified with a BRCA VUS; and 3) $40 \%$ facilitating BRCA testing for minor children. Conclusions: Results highlight the need to improve hereditary breast cancer service delivery within Florida. Findings also reveal opportunities for partnerships between genetics and non-genetics professionals to facilitate outreach and education, with the goal of positively impacting patient care.

\section{The Misdiagnosis of a Rare Disease: The Journey to a Hermansky-Pudlak Syndrome Diagnosis}

\section{Giannetti ${ }^{1}$, A. Greb ${ }^{2}$, N. Frye ${ }^{3}$, D. Appell ${ }^{4}$}

1. GeneScreen

2. Sarah Lawrence College

3. Long Island University

4. The Hermansky-Pudlak Syndrome Network Inc. 
Hermansky-Pudlak Syndrome (HPS) is improperly diagnosed despite its common prevalence of 1 in 1,800 in the Puerto Rican population. The purpose of this study was to explore why patients with HPS do not receive a proper diagnosis. Participants were 18 years or older and had a self-reported diagnosis of HPS in themselves or their child. Recruitment was at a national HPS meeting and electronically through the HPS Network Inc. The survey included 42 questions about demographics, family history, path to diagnosis, physician knowledge and patient experiences. Seventy-nine completed surveys were analyzed representing 50 adults and 29 children with HPS. Forty-five percent were from Puerto Rico. Forty-six percent reported a diagnosis of HPS type 1; $29 \%$ did not know their HPS diagnosis. The mean age of onset for nose bleeds and/or severe bleeding was 7.3 years, lung issues 24.6 years, gastrointestinal problems 16.6 years and eye problems 0.89 years. Ninety-nine percent reported eye problems. Eighty-nine percent experienced two or more HPS-associated symptoms when diagnosed; $61 \%$ reported albinism, bruising and eye problems. Seventy-six percent were misdiagnosed before receiving a diagnosis of HPS, commonly albinism (62\%). The mean number of years from first symptom to diagnosis was 16 . Fifty-two percent saw one to three specialists before being diagnosed; $28 \%$ saw four to six specialists. Self-referral to the diagnosing specialist was common (24\%). Hematologists (37\%) and genetics professionals $(24 \%)$ were felt to know the most about HPS. Of those seen by an ophthalmologist when first experiencing symptoms, $13 \%$ received a diagnosis. Only $36 \%$ received information or referral to a support group. Improvements are needed in the early and accurate diagnosis of HPS. Increasing medical specialists' awareness of HPS and related symptoms is an important first step. Ophthalmologists need more education since a majority (99\%) of individuals have eye issues starting as early as birth.

\section{Genetic Testing without Genetic Counselors: Exploring the BRCA Testing Experiences of Patients with Breast Cancer}

\section{Hayes ${ }^{1}$, L. Mandel ${ }^{2}$, K. King Mahan ${ }^{3}$, S. Mecsas-Faxon ${ }^{4}$, K. Mahoney Shannon ${ }^{5}$}

\section{Brandeis University}

2. Regis College

3. Patient Advocate

4. Harvard Vanguard Medical Associates

5. Massachusetts General Hospital Cancer Center

Increasingly, healthcare providers without expertise in genetics or genetic counseling are ordering genetic tests for their patients. It is unclear whether the inclusion or exclusion of a board certified genetic counselor in the testing process has an impact on the experience or outcome of genetic testing. The purpose of this study was to explore the experiences of women diagnosed with breast cancer who pursued BRCA testing without meeting with a board certified genetic counselor. Participants were recruited from the Mass General Cancer Center Multidisciplinary Breast Cancer Clinic and through the Facing Our Risk of Cancer Empowered (FORCE) website to participate in a semi-structured interview focused on the process they went through to obtain genetic testing, their understanding of their test results, and what they valued in the genetic testing process. The interview transcripts were analyzed using Atlas.ti software and content analysis. A total of four women participated in the study. Participants indicated a belief that the value of genetic testing lay primarily in its influence on surgical options and clarifying future cancer risks. Participants indicated that their experiences with the genetic testing process in general were positive, and that they valued the fact that the testing process was very personal when their individual physician ordered the test. Our results demonstrated, however, that there were gaps related to physician knowledge about the implications of genetic test results on medical management (outside of breast cancer surgery options) and physician discussion of the impact of test results on family members. This pilot study suggests that a BRCA testing process that does not include the involvement of a genetic counselor is not contraindicated. However, additional studies with larger and more diverse groups of patients are needed to further explore the specific areas in which the involvement of genetic counselors may enhance the testing experience.

\section{One Family, One Counselor: Continuity of Genetics Care in a Fetal Health Center}

\section{J. Kussmann ${ }^{1}$}

\section{Children's Mercy Hospital}

The Fetal Health Center at Children's Mercy Hospital provides multi-disciplinary care for women pregnant with an anomalous fetus. All patients meet with perinatology, neonatology, genetics and social work. Genetic counseling is provided to every patient at our center. This case illustrates the benefit of continuity of genetics care during pregnancy and the neonatal period. A 29 year old G3P2 woman was referred at 32 5/7 weeks gestation for consultation due to multiple congenital malformation including bilateral cleft lip, congenital heart defect and hydronephrosis. Prenatal genetic counseling regarding the high likelihood of a genetic syndrome was provided. The patient returned for an integrated consultation with neonatology, plastic surgery, cardiology, social work, genetics and radiology. The baby was born at 39 weeks gestation. He was noted to have bilateral cleft lip and palate, complex heart defect, hydronephrosis, cryptorchidism and bilateral microophthalmia with colobomas. Multiple evaluations and diagnostic tests were initiated on day of life one. The diagnostic work up concluded at 2 months of age when a heterozygous nonsense mutation c.1234C $>\mathrm{T}$ was identified in the CHD7 gene confirming the diagnosis of CHARGE syndrome. The family met with the same genetic counselor twice in the prenatal period to build rapport, gather information and explain the diagnostic process. Shortly after delivery, the family paged the genetic counselor and anxiously awaited genetic consultation. The genetic counselor met with the family four times during the diagnostic phase in the NICU. At the time of diagnosis, the family identified the genetic counselor as the only member of the team who was with them throughout their journey. The consistency reassured them that the team was doing their best to make a diagnosis. This also allowed them to feel comfortable expressing difficult, complex emotions and engaging in difficult conversations. Building good rapport is time consuming but is tremendously helpful with families who endure months of diagnostic testing.

Ethical Patient Care through Financial Means: Requiring Genetic Counseling Code for Billing Prenatal Genetic Testing

\section{Leach}

1. National Center for Prenatal \& Postnatal Down Syndrome Resources, University of Kentucky 
The past several years have seen a revolution in prenatal genetic testing. Professional statements now recommend offering noninvasive prenatal screening, reporting of incidental findings, and microarray as a first-tier diagnostic test. Each of these statements recognize the need for proper patient couseling to ensure informed consent in accepting the testing and then post-test counseling to understand the test results. But a persistent gap remains in patient care: actually providing genetic counseling. "Cost" is often cited as a reason that genetic counseling either is not provided or provided in an ad hoc fashion. Each of these prenatal genetic tests have billing codes for reimbursement through public and private insurers premised on the professional statements. One way to bridge the gap in testing being provided but not the needed genetic counseling is to condition reimbursement for prenatal genetic testing upon submission of codes for genetic counseling. This presentation will address these issues and propose a method for ensuring genetic counseling is provided - and paid for - in the administration of prenatal genetic testing.

\title{
The Implementation of a Multidisciplinary Care Clinic for von Hippel-Lindau Disease at the University of Alabama at Birmingham
}

\author{
A. Mariano ${ }^{1}$, C. Hurst ${ }^{1}$, B. Korf ${ }^{2}$ \\ 1. University of Alabama at Birmingham \\ 2. Department of Genetics, University of Alabama at Birmingham
}

Von Hippel-Lindau (VHL) disease is a tumor susceptibility syndrome characterized by hemangioblastomas, pheochromocytomas, endolymphatic sac tumors, and an increased risk of developing renal cell carcinoma and pancreatic cysts. Based on a review of the literature, we expect that multidisciplinary care will enable more thorough screening for tumors, more timely and appropriate interventions, the identification of at-risk family members, and comprehensive, coordinated care. Here we describe the process of becoming a VHL Clinical Care Center through the VHL Alliance, the approach used to identify specialists for our clinic, and our model for providing care. Our experience may help inform other providers who are looking to establish or improve a similar multidisciplinary clinic, which could be applied to the management of various diseases.

\section{Cost Should Not Be a Barrier to Genetic Testing in Patients with Paragangliomas and Pheochromocytomas}

\author{
S. Merrill ${ }^{1}$, J. Dolinsky ${ }^{2}$, S. Witherington ${ }^{2}$, J. Thompson ${ }^{2}$, L. Fishbein ${ }^{1}$, K. Nathanson ${ }^{1}$
}

1. Hospital of the University of Pennsylvania

2. Ambry Genetics

Paragangliomas and pheochromocytomas (PGL/PCCs) are rare tumors of the autonomic nervous system. In published retrospective clinical cohorts, $\sim 20-40 \%$ of patients with a confirmed diagnosis of PGL/PCC have detectable germline mutations. Identifying a genetic predisposition significantly impacts medical management of the proband and family members. Thus, consideration of genetic testing has been recommended for all patients with a PGL/PCC, regardless of family history. However, anecdotally and based on published assessments of barriers to testing, patients and physicians view cost as a barrier. To our knowledge, there has been no systematic assessment of this barrier for PGL/PCC genetic testing. We hypothesize that for the majority of patients with insurance, out-of-pocket cost is not a barrier to genetic testing for PGL/PCCs. We retrospectively reviewed data from 562 samples that were submitted to Ambry Genetics for either a PGL panel (SDHAF2, SDHB, SDHC, SDHD, TMEM127 \pm MAX, SDHA, VHL, NF1, RET) or PGL single gene analysis between August 2011 and April 2014. Samples submitted for RET, VHL, or NF1 single gene analysis only or for known familial mutation testing were excluded. A pathogenic alteration was identified in $\sim 25 \%$ of total samples tested, consistent with previously published mutation detection rates. Of the samples submitted, 32 were cancelled prior to initiation of testing due to financial reasons, including 10 with genetic testing exclusions on the insurance plan, 4 denied coverage based on lack of medical necessity, and 18 with an out-of-pocket cost higher than the patient was willing to pay. Therefore, $5.7 \%$ of samples submitted were cancelled due to financial reasons. This descriptive study confirms that at least 1 in 4 patients undergoing genetic testing for PGL/PCC have a clinically significant result and provides evidence that financial barriers should not hinder the adoption of genetic testing as standard of care for all patients with PGL/PCC.

\section{Developing a Specialty Clinic for Patients with PTEN Hamartoma Tumor Syndrome: Benefits and Barriers}

\author{
J. Mester ${ }^{1}$, C. Eng ${ }^{2}$ \\ 1. Cleveland Clinic Foundation \\ 2. Cleveland Clinic
}

Introduction: PTEN Hamartoma Tumor syndrome (PHTS) causes early-onset neoplasia and developmental disorders. Cleveland Clinic (CC) has housed a large international PHTS study since 2005. In late 2011 we established a formal PHTS multidisciplinary clinic. We review patients seen, benefits, and barriers. Methods: EPIC medical records were reviewed for patients with 1st PHTS visit after Jan. 1, 2012. Before scheduling a genetic counselor (GC) clinic coordinator interviewed patients/parents and gathered medical records to identify needed specialty appointments. Financial clearance was also required. GC visits were billed using CPT code 96040 . Demographics, 96040 billing, number/type of specialists seen, and ensuing procedures, images, and management changes were documented. Descriptive statistics and 2-tailed t-tests were employed. Results: 38 PHTS patients met inclusion criteria. $22(58 \%)$ were female and $4(11 \%)$ were seen pre-and-post-testing. Mean/median years at visit and diagnosis were 24/24.5 and 22/19.5 respectively. Five lived $<2 \mathrm{~h}$ of CC; $53 \%$ traveled by plane. 29/38 (76\%) self-referred after viewing the clinic website; others were referred by outside genetics teams or CC specialists. Among 32 non-regular-CC patients, 24 visited once and 8 had $>1$ visits. Mean/median 96040 units billed were $3.9 / 4$. Adult women $(n=13)$ saw the most specialists (mean 4.2 vs. $2.8, p=0.03)$. Frequent specialties included endocrine ( $84 \%)$ and gastroenterology $(42 \%) ; 62 \%$ adult females saw high-risk breast and $46 \%$ visited GYN-oncology. 18/38 (47\%) and 32 (84 \%) had 1 or more procedure or imaging study respectively. $84 \%$ received recommendations that directly altered care. Patients not yet part of PTEN research enrolled; $61 \%$ enrolled in neurodevelopmental research. 14 families received travel vouchers. Conclusions: PHTS clinic allows patients to see specialists experienced in their disorder and participate in research. A 
GC is effective as coordinator of syndrome/gene-specific clinics; downstream revenue may support this position. Travel grants exist but resources for visit costs are needed.

Impact of Genetic Counseling in the Cardiac Intensive Care Unit for Infants with Isolated Congenital Heart Defects

R. Palmquist ${ }^{1}$, S. Bleyl ${ }^{2}$, E. Rothwell ${ }^{3}$, S. Fitzgerald-Butt ${ }^{4}$, S. Latimer ${ }^{4}$, L. Meyers ${ }^{5}$

1. University of Utah

2. Intermountain Healthcare and University of Utah

3. University of Utah College of Nursing

4. Nationwide Children's Hospital

5. Primary Children's Hospital

Inpatient genetic counseling for infants with both syndromic and isolated congenital heart defects (CHD) was implemented in the Cardiac Intensive Care Unit (CICU) at Primary Children's Hospital $(\mathrm{PCH})$ over the past 3 years. Genetic counseling services for isolated CHD is a fairly new practice, and the impacts have not been well studied. We sought to determine if inclusion of genetic counseling in the care process had an impact on parents' understanding of recurrence risk or cause, on psychosocial factors, including anxiety and stress, and on parent's satisfaction with the information received. A multi-measure questionnaire was mailed (or sent) to parents of infants with a CHD seen in the PCH CICU. Responses were compared between two groups; parents who received genetic counseling (GC-group) and parents who did not receive genetic counseling (non-GC group). While there was no significant difference in parent's understanding of recurrence risk or cause between the two groups, parents who received genetic counseling for their child's CHD reported higher satisfaction with the information they received. Limited ability to report recurrence risk in both groups and parent's responses to open-ended questions support the use of written materials and visual aids to help communicate recurrence risk and cause. In addition, it may be beneficial to consider timing and location of counseling in order to provide this counseling during the least stressful period of inpatient stays.

\title{
Service Delivery Model and Experiences in a Genetics Clinic for an Underserved Population
}

\author{
J. Profato $^{1 *}$, A. Woodson ${ }^{1 *}$, N. Elsayegh ${ }^{1}$, S. Rizvi $^{1}$, A. Rieber ${ }^{1}$, B. Arun ${ }^{1}$ \\ 1. The University of Texas MD Anderson Cancer Center \\ *The first two authors contributed equally to this work.
}

Individuals identified with a BRCA mutation benefit from increased screening and prevention guidelines. Mutations in $B R C A 1$ and $B R C A 2$ have been identified across several ethnic groups, including underserved populations; therefore, some studies have been conducted to determine the utilization of genetic services for these patients and have shown a positive impact. Studies have demonstrated the limited access to genetic services for underserved populations; however, there is limited data in regards to suggested solutions to this disparity in healthcare. This study describes the successful pilot implementation of a group counseling based breast cancer genetics clinic for Hereditary Breast and Ovarian Cancer (HBOC) that provided services to an underserved population at a community hospital in Houston, TX. Group counseling was provided via a bilingual HBOC educational video, and one-onone family history and risk assessment was provided to each patient. One hundred and fifty-one patients received genetic counseling (62\% Hispanic; $23 \%$ African American, $12 \%$ Caucasian, $3 \%$ Asian/Pacific Islander), and 131 patients (87\%) underwent BRCA1 and BRCA2 genetic testing. Eleven patients $(8 \%)$ were identified to have BRCA mutations and since then, the majority is undergoing preventative surgery and/or screening. In-person results disclosures were initially utilized and were provided within an average of 40.06 days. Telephone disclosures were then implemented, resulting in a reduction of time until disclosure to an average of 14.58 days. This clinic was a unique example of the establishment, structure, and operation of HBOC genetic services in an underserved population. Group-based counseling in this setting may most efficiently provide services to an increased number of patients while conserving limited resources. Flexibility to accept alternative infrastructure and delivery model of the clinic were the key to success and may serve as an example for genetic services in similar community based settings.

\section{Centering Pregnancy: An Untapped Delivery Care Model for Genetic Counseling}

\section{P. Robbins Furman ${ }^{*}$, S. J. Noblin ${ }^{2 *}$}

1. Baylor College of Medicine

2. The University of Texas Health Science Center at Houston

*The first two authors contributed equally to this work.

The Centering Pregnancy model of prenatal care was initially envisioned and described by nurse-midwife Sharon Schindler Rising in 1998. Most studies looking at the benefits of this model of group prenatal care have shown improved birth outcomes and increased patient satisfaction. Participants in Centering Pregnancy receive group prenatal care with 8-12 other women at a similar gestational age. After self-assessments and assessments with the obstetric provider, the women participate in facilitated discussions. Topics for discussion vary by gestational age and include nutrition in pregnancy, common discomforts, labor and delivery, parenting and infant care. The initial session at 12-16 weeks includes the topic of prenatal testing. While many clinics that provide Centering Pregnancy have specialists such as dieticians and social workers who facilitate discussion topics, historically genetic counselors have not been involved. We report on the experience of genetic counselors working in underserved populations in Houston, TX who have had the opportunity to facilitate group discussions on prenatal screening and testing options in Centering Pregnancy groups. During the discussion of prenatal screening and testing, Centering participants are encouraged to verbalize their understanding of and concerns about genetic screening with the group. They are encouraged to ask questions and/or report on their past experiences with screening. Having a genetic counselor facilitate the prenatal testing discussion can result in informed decision-making about available prenatal tests. Additionally, becoming acquainted with the clinic's genetic counselor in a non-acute setting may make patients less anxious should a formal genetic counseling appointment be necessary. Benefits for the genetic counselors 
include increased visibility as health care providers in the primary care setting. Centering Pregnancy is an as of yet untapped delivery care model that genetic counselors should explore.

Population Screening for Hereditary Cancer, Does it Really Work for Everyone?

\title{
L. Robinson ${ }^{1}$, S. Pirzadeh-Miller ${ }^{2}$
}

1. The University of Texas Southwestern Medical Center

2. UT Southwestern Simmons Cancer Center

Population screening for the Lynch syndrome is routinely done and screening for Hereditary Breast and Ovarian Cancer syndrome (HBOC) is now recommended on all woman based on the U.S. Preventive Services Task Force recommendations. The reduction in cancer is based on the mutation detection rate, uptake of prophylactic surgery, and compliance with the National Comprehensive Care Network (NCCN) guidelines. Although many centers have instituted these programs, very few have published outcome measures and no one has modeled the long term effects of screening with data on patient compliance. The aim of our study was to determine if population cancer genetic screening will ultimately lower the cancer incidence. We created a computer model to determine the cancer reduction rate over 30 years from our screening program comparing a private and underserved population. We looked at 96,055 patients undergoing mammography screening who were screened for HBOC and 294 colon/uterine tumors over the course of 20 months. The mutation detection rate for HBOC screening in the mammogram populations was $42 \%$ in the private setting and $28 \%$ in the underserved community. The uptake for prophylactic mastectomy and oophorectomy was $38 \% / 86 \%$ in the private population and $20 \% / 22 \%$ in the underserved population. Patient compliance the NCCN guidelines for HBOC management was $74 \%$ in private and $39 \%$ in the underserved. Using this data we modeled the effect on the cancer incidence over 30 years and found that there would be at least a $19.12 \%$ reduction in breast cancer and $33.92 \%$ reduction in ovarian cancer in the private population and $5.94 \% / 5.76 \%$ in the underserved respectively. For Lynch syndrome screening we found a $51 \%$ reduction in colon cancer incidence in the private population and $46 \%$ reduction in the underserved based on an estimated $100 \%$ mutation detection rate and $90 \% / 81 \%$ NCCN management compliance rate in the respective groups. In conclusion, population screening will reduce the cancer incidence, and modeling can be used as a quality measure for genetic counselors, to identify areas for improvement, disparity and to predict cost savings.

\section{Jewish Genetic Disease Carrier Screening in Atlanta: Success of Marketing and Outreach Campaigns}

\author{
Y. Shao ${ }^{1}$, S. Liu' ${ }^{2}$, K. Grinzaid ${ }^{3}$ \\ 1. Emory University \\ 2. Rollins School of Public Health, Emory University \\ 3. Emory Genetics Lab
}

Introduction: The Atlanta Jewish Gene Screen (AJGS) was the first 2 year pilot program in the Metropolitan Atlanta area to promote and facilitate preconception carrier screening for 19 genetic diseases with a high carrier rate in the Ashkenazi Jewish population. The program included extensive marketing and outreach campaigns, and a variety of educational and promotional efforts. Purpose: The purpose of this study was to assess carrier frequencies in the Atlanta Jewish population as compared to those reported previously in the U.S., to identify the demographics of participants, and to evaluate marketing and outreach campaigns. Methods: We performed a retrospective analysis of de-identified data collected on 724 participants through medical chart review and questionnaires. The Fisher's exact test compared carrier frequencies to the published literature. Age, marital status, number of Jewish grandparents, pregnancy history, and information on how participants heard about the program were summarized using descriptive statistics. Logistic regression analysis identified demographic characteristics associated with a willingness to spread the word about the importance of screening. Results: The overall carrier frequency and the disease-specific carrier frequencies for 17 of the 19 diseases were consistent with published literature. The majority of participants were screened prior to conception ( $93.4 \%$ ) and reported $100 \%$ AJ ancestry (72.5 \%). Friends, rabbis and family members were the most common influencers in participants' screening decisions. The willingness to influence others was higher in participants who were older $(p<0.02)$, had a pregnancy history $(p<0.01)$, and who had been previously screened $(p<0.002)$. Conclusions: The carrier frequencies in the Atlanta Jewish community are generally consistent with those of other Ashkenazi Jewish populations in the U.S. The analysis also found that educating influencers and increasing overall awareness among potential participants were the most effective outreach strategies.

\section{High Frequency of Genetic Services for Patients with Inborn Errors of Metabolism}

\author{
1. Sanford Health \\ 2. Children's Hospital of Pittsburgh \\ 3. University of Minnesota \\ 4. Michigan Public Health Institute
}

Q. Stein ${ }^{1}$, C. Walsh Vockley ${ }^{2}$, S. Berry ${ }^{3}$, S. Zhai ${ }^{4}$, S. J. Hiner ${ }^{4}$

The Inborn Errors of Metabolism Collaborative (IBEMC) consists of clinicians from 26 institutions collecting data to enhance understanding of metabolic conditions diagnosable by newborn screening. Member institutions enter data about individually rare but collectively common conditions, including clinical interactions, hospitalizations, and lab testing. Through the registry, established in 2007, baseline data is now available. We analyzed data for (1) provision of genetic counseling and (2) utilization of genetic testing. In the registry, 1,138 subjects had values for the question "Has family received genetic counseling?" Of these, 1,091 (95.9\%) reported genetic counseling had been provided. A question added to the registry recently asks who provided the genetic counseling, with data recorded for 214 subjects. In 144 (67.3\%) cases, a genetic counselor was involved in the counseling while in 70 cases $(32.3 \%$ ) the counseling was provided by a physician, nurse practitioner, dietician, or medical student. Thus, while the majority of families are receiving genetic counseling, in about $1 / 3$ of cases a genetic counselor did not provide the service. Genetic test utilization was also assessed. 
In the registry, 684 (67.7 \%) of 1,010 subjects had undergone DNA testing, with usage especially high for fatty acid oxidation disorders. Additionally, average age of individuals in the registry who have had genetic testing is younger ( 7.4 years) than those who have not (10.9 years). This may reflect an increase in use of genetic testing following newborn screening, especially for conditions in which testing may assist with diagnostic confirmation; supporting data will be presented. In summary, within the IBEMC there is very high frequency of genetic counseling for metabolic conditions, though in $1 / 3$ of cases a genetic counselor is not involved. Additionally, while metabolic conditions have historically been diagnosed by biochemical methods, there is now high usage of DNA testing for these conditions. The intrinsic relevance to the genetic counseling profession will be discussed.

\title{
Genetic Testing Practices of Physicians for Primary Immunodeficiency Diseases: Are There Unmet Patient Needs?
}

\author{
S. Walterman' ${ }^{1}$ H. He ${ }^{2}$, J. Connor ${ }^{2}$, R. Marsh', K. Zhang ${ }^{1,2}$ \\ 1. University of Cincinnati \\ 2. Cincinnati Children's Hospital Medical Center
}

Primary immunodeficiency diseases (PIDs) encompass a variety of genetic disorders that involve an inborn defect in the immune system. An estimated 1 in 1,200 persons in the United States have a diagnosed PID. The goals of this study were to describe current genetic testing practices of physicians for patients with PIDs and to determine if there are unmet patient needs for genetic services. We invited members of the Clinical Immunology Society and the Histiocyte Society, organizations expected to include physicians who treat patients with PIDs, to complete a survey designed to assess 1) demographics, 2) the frequency genetic testing is ordered, 3) the most common tests ordered, 4) the reasons for testing, 5) the perceived disadvantages and barriers to testing, and 6) the frequency of genetic counseling referrals. Descriptive statistics, the Cochran-Armitage trend test, and linear-by-linear association tests were used to characterize respondents and assess potential factors influencing their practices. We found that $51 \%$ of respondents ordered genetic testing for up to only $20 \%$ of patients suspected to have PIDs. Disparities exist between the frequency with which some disorders are treated and tested. The biggest perceived disadvantage associated with genetic testing was the possibility of uncertain test result interpretation. Almost all had access to genetic counselors, but $41 \%$ did not refer any patients to counseling before ordering testing. The frequency of counseling referrals was not influenced by selfreported knowledge of genetics. Results indicate an expansion of available genetic testing options is necessary to better meet the needs of PID providers and to help further incorporate testing into clinical practice. Increased collaboration between PID providers and genetic professionals is needed to increase awareness among PID providers of counseling issues and to reduce the burden of interpreting uncertain test results and providing genetic counseling.

\section{Receipt of Cancer Genetics Services among Young Breast Cancer Survivors in Georgia}

R. Webster' ${ }^{1}$, T. Zhang', M. Martine ${ }^{3}$, C. Bellcross ${ }^{1}$

1. Emory University School of Medicine

2. Rollins School of Public Health, Emory University

3. Georgia Center for Oncology Research and Education

Introduction: Women diagnosed with breast cancer before age 50 meet the National Comprehensive Cancer Network (NCCN) referral criteria for cancer genetic counseling. Ensuring these women are identified and offered risk assessment for a possible hereditary cancer syndrome is essential to providing cancer screening and prevention strategies to appropriate individuals and their families. However, limited evidence suggests under-referral and low uptake of these services in this demographic. As part of a Centers for Disease Control and Prevention Cooperative Agreement, we assessed receipt of cancer genetic services among young breast cancer survivors in Georgia. Methods: A 47 item anonymous survey was administered to young breast cancer survivors associated with Georgia breast cancer support groups. Questions centered on women's experience with referral and receipt of cancer genetic services, including facilitators and barriers, and beliefs regarding family cancer risk. Results: Eligible responses were received from 292 individuals. Less than half of respondents reported cancer genetic counseling recommendations, and only $39.7 \%$ received these services. Uptake motivators included desire to know future cancer risk (38.2\%), cancer treatment decisions (29.1\%), and to benefit family members (19.1\%). Inadequate insurance coverage was the most common barrier reported (28.6\%). Although all participants were diagnosed with breast cancer at an early age, only $16.6 \%$ of women chose to have genetic testing. Surprisingly, when questioned regarding their perception of breast cancer risk in their family, $75.8 \%$ of respondents selected 'same' or 'lower' risk compared to the general population. Conclusions: Although all women surveyed met NCCN criteria for cancer genetic services, the majority appear unaware of its importance to their long-term survival. Our findings suggest strengthening collaboration between cancer survivors, healthcare providers, and policy makers to improve referral and receipt of cancer genetic services among young breast cancer survivors.

\section{Adult}

\section{Assessment of the Clinical Presentation of Females Heterozygous for Fabry Disease: A Comparison of Classic and Later-Onset Forms}

\author{
A. Conner ${ }^{1}$, D. Doheny ${ }^{1}$, J. Martignetti $^{1}$, R. Desnick ${ }^{1}$
}

\section{Icahn School of Medicine at Mount Sinai}

Fabry disease is an X-linked lysosomal storage disorder that affects multiple organs throughout the body. The disease is caused by a deficiency in the alpha-galactosidase A enzyme (alpha-Gal A), which is responsible for the breakdown of globotriaosylceramide (GL-3) in lysosomes. This accumulation of GL-3 in tissues can affect the cardiac, gastrointestinal, excretory, and pulmonary systems, which may result in debilitating and life-threatening complications. Classically affected males (with $<1 \%$ of normal alpha-Gal A activity) present in childhood or adolescence with acroparesthesias, angiokeratomas, corneal/lenticular opacities and hypohidrosis. With age, progressive renal failure and cerebrovascular and cardiovascular complications 
lead to early demise. Males with the later-onset phenotype have residual alpha-Gal A activity $(>1 \%)$ and typically present in adulthood. Clinical manifestations in heterozygous females of both the classic and later-onset phenotypes are variable due to random X-chromosomal inactivation, ranging from asymptomatic to as severe as affected males. However, clinical studies to date have clustered these women into a single group without differentiation between those with classic and later-onset phenotypes. This study examines the differences in the clinical presentation between classic and later-onset heterozygous females. A quantitative analysis of data obtained from a self-reported questionnaire of 28 heterozygous females compared clinical manifestations, Fabry pain, quality of life and reproductive decision-making between phenotype groups. This study found differences in some clinical symptoms, Fabry pain, age of onset of Fabry pain and disease perception between the two phenotype groups. Data could be informative in drafting guidelines for treatment of females in both phenotypes.

\title{
De Novo Mutation Rate in the RYR2 Gene: Implications for Genetic Counseling
}

K. Davis ${ }^{1}$, T. Hart ${ }^{2}$, C. Antolik ${ }^{2}$, D. Macaya ${ }^{2}$

1. Genetic Counseling Program, Johns Hopkins University/National Human Genome Research Institute

2. GeneDx

The proportion of mutations that arise de novo in disease-associated genes is often unknown. This may lead to difficulty in classifying variants, understanding their potential pathogenicity, and estimating risk for family members. The RYR 2 gene, in which mutations occur de novo in 20 to $40 \%$ of cases of catecholaminergic polymorphic ventricular tachycardia (CPVT), is a comparatively well-studied example of these difficulties. The RYR2 gene encodes the cardiac Ryanodine Receptor, which is a calcium channel localized to the sarcoplasmic reticulum (SR) membrane in cardiomyocytes. This channel mediates calcium release from the SR and regulates excitation-contraction coupling. Mutations in RYR2 lead to 50-55\% of cases of CPVT, while mutations in the RYR2 gene associated with arrhythmogenic right ventricular cardiomyopathy (ARVC) are rare. To directly assess the rate of de novo mutation in RYR2, the molecular results obtained from families that were tested at GeneDx from 2010-2014 were reviewed to determine whether an $R Y R 2$ variant identified in a proband was inherited (informative cases, $N=87$ ). The $R Y R 2$ de novo mutation rate was $43.5 \%$. While other factors such as non-paternity, gonadal mosaicism, and allele dropout may mimic the observation of an apparently de novo mutation in a proband, these events are likely to be comparatively rare. Among all de novo disease-causing mutations, $93.3 \%$ fell within one of the mutational hotspots described in the literature. These data are consistent with previous studies suggesting that the de novo mutation rate for the RYR2 gene is greater than that reported for other genes. Knowledge of the de novo mutation rate for the RYR2 gene has practical implications for genetic counseling of the proband and his/her family, and can provide vital insight regarding pathogenicity and recurrence risk for other family members. Of note, in the event of an apparent de novo mutation, families should also be counseled that future pregnancies are still at increased recurrence risk due to the possibility of gonadal mosaicism.

\section{The Use of Social Media and the Impact of Support on the Well-Being of Adult Cystic Fibrosis Patients}

\author{
M. Faust ${ }^{1}$, A. Sellers ${ }^{1}$, S. Wienke ${ }^{2}$, A. Eshbaugh ${ }^{3}$, A. Rainwater ${ }^{4}$ \\ 1. University of South Carolina \\ 2. The Medical University of South Carolina \\ 3. The Cystic Fibrosis Foundation \\ 4. LifeCARE Psychology Group, LLC
}

Introduction: Cystic fibrosis $(\mathrm{CF})$ is a chronic, genetic disorder that requires daily treatment of symptoms, the stress of which can impact emotional wellbeing. Due to potential cross-contamination of bacterial infections, individuals with CF are discouraged from direct interactions and must find other venues to receive support, such as social media. Current literature is lacking research that examines adult CF patients' utility of online support groups and social media. Hypothesis: We proposed a positive correlation between the amount of time these patients spend on CF-related social media sites and perceived value of these sites for emotional support. Methods: A survey with demographic questions and a series of multiple choice and Likert scale questions was designed to assess the participants' well-being and use of social media. The survey was posted on social media sites to identify trends between the patient's use of social media and their emotional wellbeing. Data from 103 adult participants with CF were used for statistical analysis, including Chi-square for association, Spearman's rho correlations, and ANOVA for dependency. Qualitative data was collected through 15 telephone interviews, coded, and analyzed using grounded theory methods. Results: Participants reported using social media sites on average less than 2 hours per week. The majority of participants $(85 \%)$ were actively posting on these sites. Qualitative analysis identified five major themes of their use of social media: (1) choice of sites; (2) feelings of isolation due to CF; (3) use for medical information; (4) emotional support; and (5) negative experiences. Conclusions: Individuals with CF are using social media sites for CF-related support. We found a positive emotional response to social media with increased use and interaction $(p<0.001)$. Significantly negative correlations of the use of social media include patient's perceived treatment burden $(p=$ $0.001)$ and CF-related physical symptoms requiring therapy $(p=0.002)$.

A Role for Preventative Genetics? The Impact of FTO Results on Intention to Lose Weight

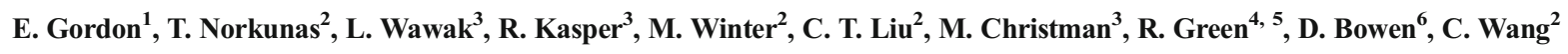

1. Invitae

2. Boston University School of Public Health

3. Coriell Institute for Medical Research

4. Brigham and Women's Hospital

5. Harvard Medical School

6. University of Washington 
Approximately $69 \%$ of adults in the U.S. are categorized as overweight or obese. Although it has been suggested that genetic risk information for obesity could help motivate healthy behaviors, evidence to support this has been limited. We conducted a randomized trial to examine the short-term impact of providing personalized risk feedback for obesity, based on the FTO variant (rs9939609) and lifestyle information (time spent sitting watching TV) using a $2 \times 2$ factorial design. Study participants were recruited from the Coriell Personalized Medicine Collaborative (CPMC), and randomized to receive either 1) genetic risk only, 2) lifestyle risk only, 3) both genetic and lifestyle risks, or 4) no risk feedback (NRF). Participants completed surveys at baseline and 3 month follow-up. Linear regression models were run to examine the impact of risk feedback on intentions to lose weight at follow-up. A total of 696 participants completed both baseline and 3-month follow-up surveys. Mean age of participants was 48 years, $71 \%$ were female, and $61 \%$ had a BMI of 25 or greater (overweight/obese). In this study, we focused on the 387 participants who received either elevated risk feedback for obesity based on genetics, alone $\left(G \_\right.$high) or in combination with lifestyle risk information ( $G \_$high $+L_{-}$low, $G \_$high $+L_{-}$high) or NRF. A significant difference was observed for intention to lose weight among participants who received $\mathrm{G}$ high vs $\mathrm{NRF}(p=0.01)$. Those receiving $\mathrm{G}$ high (Madj 3.32) or $\mathrm{G}$ high $+\mathrm{L}$ high (Madj 3.32) feedback had significantly higher intent to lose weight compared to NRF (Madj=3.02); this was not seen in the G_high+L_low feedback group (Madj= 3.16). Additional analyses revealed that the impact of genetic risk was observed among those who were normal weight, but not overweight/obese. Genetic information for obesity may be a useful tool in preventative medicine, serving as motivating factor for healthy weight maintenance in normal weight individuals. Efforts are needed to better understand the impact of receiving concordant/discordant risk information from different sources.

\title{
Examining Differences in Symptoms in Individuals with Hypermobile Ehlers-Danlos Syndrome in Relation to Puberty
}

\author{
K. Heraty ${ }^{1}$, J. Johnson ${ }^{1}$, X. Zhang ${ }^{1}$, E. Schorry ${ }^{1}$, D. Neilson ${ }^{1}$
}

\section{Cincinnati Childrens Hospital Medical Center}

Ehlers-Danlos syndrome hypermobile type (EDS-HT) is a connective tissue disorder characterized by excessive movement of the joints. Although inherited in an autosomal dominant pattern, $90 \%$ of diagnosed patients are female. Clinical observations have indicated that females with EDS-HT demonstrate an increase in symptoms around the time of puberty, whereas males often improve during this time. The difference in disability experienced and discrepancy in sex prevalence may become more pronounced at puberty implicating sex hormones as an underlying cause. This study aimed to characterize the progression of symptoms in both males and females with EDS-HT in early/pre-puberty (EPP) and late/post-puberty (LPP) and serve as the framework for further research examining the impact hormonal changes have EDS-HT symptoms. Ninety-one patients with either a clinical diagnosis of EDS-HT or a first degree relative with a diagnosis and a positive Beighton score $(>4)$ filled out questionnaires at Cincinnati Children's Hospital Medical Center's Connective Tissue Clinic. Participants were grouped into EPP and LPP and compared on their composite scores on three questionnaires. Females had significantly higher Functional Disability Inventory scores than males $(p=0.0289)$. When examining the FDI changes in males and females separately, we found that in females, FDI was significantly higher in the LPP group compared to the EPP group ( $p<0.0001$ ). Specifically, females in the LPP group were seen to have significantly higher disability related to strenuous activities. In females, the PEDSQL quality of life score related to pain was significantly lower in the LPP group $(p=0.006)$, suggesting that pain contributed to a lower quality of life. These results indicate that the overall disability related to an individual's symptoms increases.

\section{A Novel Homozygous Variant in RRM2B in Two Siblings with Mitochondrial DNA Depletion Syndrome}

\author{
W. Mu' ${ }^{1}$, M. Gunay-Aygun ${ }^{2}$ \\ 1. Johns Hopkins University \\ 2. Johns Hopkins University School of Medicine
}

$R R M 2 B$ is a nuclear-encoded mitochondrial DNA (mtDNA) repair gene responsible for supplying dNTPs for mtDNA synthesis. Mutations in $R R M 2 B$ are associated with classic findings of mtDNA depletion including sensorineural hearing loss (SNHL), progressive external ophthalmoplegia (PEO), ptosis, myopathy, ataxia, dysphagia and other gastrointestinal disturbances. Disorders of $R R M 2 B$ are further divided into two sub-phenotypes distinct in terms of inheritance, age of onset, and severity. Heterozygous mutations in $R R M 2 B$ cause adult-onset autosomal dominant disease with mean age of onset at 46 years. Symptoms are variable and include facial weakness, mild dysphagia, and fatigue. Homozygous mutations in $R R M 2 B$ result in severe infantile or early childhood-onset disease with progressive symptoms including respiratory insufficiency, renal tubulopathy, and lactic acidosis, and often reduce lifespan. We describe two sisters, ages 21 and 14, from a first-cousin union, with comparatively milder features of mtDNA depletion associated with a novel homozygous variant in $R R M 2 B, \mathrm{c} .118 \mathrm{C}>\mathrm{T}$ (p.Arg40Cys). This variant is predicted to be deleterious, via in silico studies, by preventing the formation of an iron-binding salt bridge. Both probands were asymptomatic until age 5 when they presented with SNHL; they now manifest PEO, ptosis, retinal degeneration, decreased subcutaneous fat, muscle atrophy, and dysphagia. The 21-year-old also has polyneuropathy and depression. Muscle biopsy at age 18 showed ragged red fibers and she was initially diagnosed as having Kearns-Sayre syndrome (KSS). With a milder presentation in comparison to previously published cases with homozygous mutations, these patients expand the phenotype of RRM2B-associated mtDNA depletion syndrome and emphasize the importance of molecular diagnosis in PEO and KSS. In addition, the novel p.Arg40Cys mutation in this family point to the functional significance of this iron binding domain; future studies may lead to treatment targeting this site.

\section{The Complexity Continues: Identification of Pathogenic Sarcomeric Mutations in Families with a Clinical Diagnosis of Arrhythmogenic Right Ventricular Cardiomyopathy}

\section{B. Murray ${ }^{1}$, C. James ${ }^{1}$, C. Tichnell ${ }^{1}$, H. Calkins ${ }^{1}$, D. Judge ${ }^{1}$}

\section{Johns Hopkins Hospital}

Introduction: Arrhythmogenic right ventricular cardiomyopathy (ARVC) is an inherited cardiomyopathy characterized by ventricular arrhythmias and sudden death. Currently $60 \%$ of patients meeting Task Force Criteria (TFC) have an identifiable mutation in one of the desmosomal genes. Because of 
the importance of identifying those at risk for sudden death, gene finding efforts have continued. Hypothesis: Whole genome sequencing (WGS) in patients with ARVC with no previously identified genetic root cause may elucidate candidate genes linked to disease. Methods: Twenty five (25) individuals from 18 families who previously had no identified mutation on clinical genetic testing for the 5 desmosomal genes and TMEM43 associated with ARVC were consented for WGS at John's Hopkins University (JHU). All individuals met clinical TFC for ARVC. Variation was analyzed and assessed for pathogenicity by clinical geneticists, and possible candidate genes reported. Furthermore, a group of 10 patients presenting for clinical genetic testing (consented to the JHU ARVC registry) with previous negative genetic results had expanded genetic testing that included additional clinically available candidate genes. Results: In the initial 18 families, mutations in sarcomeric genes were identified in $4(22 \%)$ families in $M Y B P C 3$ : one novel intronic deletion and three novel pathogenic missense mutations. Once sarcomeric mutations were identified as possible candidates, and the 10 additional individuals had comprehensive cardiomyopathy screening including all sarcomeric genes, $2(20 \%)$ individuals were identified to carry pathogenic mutations in $M Y H 7$; one a novel pathogenic missense mutation, and one published pathogenic missense mutation. Sarcomeric mutations were identified in $33 \%$ total. Discussion: Previously, increasing overlap has been described between inherited cardiomyopathies including ARVC and dilated cardiomyopathy, and dilated cardiomyopathy and hypertrophic cardiomyopathy. This data for the first time shows pathogenic mutations in the sarcomere associated with an ARVC phenotype. This provides important additional evidence for clinical practice to recommend comprehensive cardiomyopathy gene testing to patients with ARVC.

\title{
Disclosure of Psychiatric Manifestations of 22q11.2 Deletion Syndrome: A Retrospective Chart Review Documenting Clinical Practice of Medical Geneticists
}

\author{
S. Talcott Baughman ${ }^{1}$, J. Austin ${ }^{2}$, E. Morris ${ }^{3}$ \\ 1. University of British Columbia \\ 2. Departments of Psychiatry and Medical Genetics, University of British Columbia \\ 3. Provincial Medical Genetics Program, BC Women's Hospital and Health Centre
}

Introduction: Parents of children with 22q11.2 deletion syndrome (22qDS) report receiving information about the psychiatric manifestations of 22qDS from non-medical sources. Medical geneticists (MGs, the group of healthcare providers to most often diagnose 22qDS) self-report discussing psychiatric features of 22qDS with families, though often only when the child is older. Thus, we sought to objectively determine the frequency with which MGs discuss the psychiatric manifestations of 22qDS a) in letters to referring physicians and b) with families, and to explore plans for follow up. Hypotheses: We hypothesized that the frequency with which MGs disclosed the psychiatric manifestations of 22qDS would be higher: a) in 2012 as compared to 2000 , and b) in adult/adolescent ( $>=12$ years of age) as compared to pediatric $(<12$ years of age) patients. Methods: We reviewed charts of patients referred to our genetics clinic for diagnosis and consultation for 22qDS between January 1, 2000 and December 31, 2012 and abstracted data on the features of the syndrome that were discussed with referring physicians and families at diagnosis and follow-up. Hypotheses were tested using Fisher's exact test in SPSS. Results: Our review of 127 charts showed that psychiatric disorders were discussed in consult letters to referring physicians less frequently (44\%) than all other features of 22qDS. Despite exhaustive review of charts, the content of discussions with families was typically unclear. Follow-up by MGs was infrequently suggested, and when it was, only $33 \%$ of patients returned. There was no statistical support for either of the hypotheses. Conclusion: Disclosure of psychiatric risk to families and primary care providers is necessary in order to increase awareness, so that early intervention for psychiatric disorders can be implemented to achieve a better prognosis for those affected. These empiric data offer some explanation as to why psychiatric services are underused by individuals with $22 \mathrm{qDS}$.

\section{Cancer}

\section{How Do We Counsel on Somatic Tumor Testing Reports? Red Herring or the Real Deal?}

\author{
M. Dreon ${ }^{1}$, M. Truelson ${ }^{2}$, K. Niendorf ${ }^{2}$, C. Mason $^{3}$ \\ 1. Minnesota Department of Public Health Newborn Screening Program \\ 2. University of Minnesota Medical Center, Fairview \\ 3. Department of Clinical Diagnostics, Ambry Genetics
}

Tumor genetic analysis is becoming more commonplace in an attempt to better understand cancer etiology and optimal treatment for patients. Given the implementation of somatic genetic testing in clinical care, standardized protocols are not routinely applied for informed consent and follow-up of results. The vast majority of these patients are not educated regarding the potential for obtaining hereditary information from this analysis. Family history is not frequently obtained, and patients are not routinely referred for post-test genetic counseling. Since approximately $5-10 \%$ of cancers are associated with germline mutations, cases of hereditary cancer syndrome-related mutations will be increasingly detected but potentially without involvement of genetic counseling services. We present a case of somatic tumor testing which identified a germline $B R C A 1$ mutation. The proband presented to the genetic counseling service with a sibling's tumor analysis report showing a $B R C A 1$ mutation in a lung adenocarcinoma, denoted as k519fs*13. Family history was significant for early onset breast and ovarian cancer, which was not ascertained at the time of the tumor testing. The genetic counselor obtained a cousin's BRCA testing report indicating a germline BRCA1 mutation, specifically $1675 \mathrm{delA}$. Comparison was made with standard nomenclature, and it was determined that the tumor mutation and the germline mutation were the same resulting in a hereditary breast-ovarian cancer syndrome phenotype. Germline genetic testing confirmed the presence of the BRCA1 mutation in the proband's brother, a mutation originally detected with somatic tumor genetic analysis. This case illustrates the importance of family history consideration when performing somatic genetic testing as well as involvement of genetic counseling services. Additional difficulty in clinical interpretation arises due to variations in mutation nomenclature. Genetic counselors are positioned to provide the expertise needed to assess germline and somatic genetic testing in the context of family cancer histories. 


\title{
Cancer Multi-Gene Panel Testing: Clinical Experience and Impact on Medical Management
}

\author{
M. Gabree ${ }^{1}$, G. Chan-Smutko ${ }^{2}$, L. Rodgers ${ }^{1}$, M. Seidel ${ }^{1}$, K. Shannon ${ }^{1}$, D. Patel ${ }^{3}$, E. Blouch ${ }^{2}$, J. Lawrence ${ }^{1}$
}

1. Massachusetts Genera Hospital

2. Massachusetts General Hospital Cancer Center

3. Center for Cancer Risk Assessment, Massachusetts General Hospital

Objective: Multi-gene panel testing (MGP) introduces new complexities to cancer genetic counseling. At the Massachusetts General Hospital Center for Cancer Risk Assessment (CCRA), MGP tests are ordered by genetic counselors. The National Comprehensive Cancer Network (NCCN) provides testing and management guidelines for several syndromes, including Hereditary Breast/Ovarian Cancer (BRCA1, BRCA2), Li-Fraumeni ( $p 53)$, Lynch ( $M L H 1, M S H 2, M S H 6, E P C A M, P M S 2)$, and polyposis syndromes (APC, MYH). We aimed to utilize NCCN testing criteria and medical management recommendations to define our cohort and assess the impact of MGP on management. Methods: Sixty-five cases with MGP results available were randomly selected. Case pedigrees were coded as "meets" or "does not meet" NCCN (v1.2014) genetic testing criteria for the aforementioned syndromes. Management recommendations were determined for each case based on NCCN criteria (when available) or expert opinion and literature review conducted by physicians and genetic counselors in the CCRA. Results: The type of MGP ordered varied from 18- to 41-gene panels. Forty-five cases met NCCN testing criteria for one syndrome and 7 met criteria for two syndromes. In thirteen ( $20 \%)$ cases a deleterious mutation was identified, 40 $(61.5 \%)$ were negative, and $12(18.5 \%)$ had a variant of uncertain significance (VUS). Five of the 13 mutation positive cases met NCCN testing criteria for the corresponding mutated gene. Of the 13 mutation-positive results, seven $(53.8 \%)$ resulted in medical management revisions in accordance with NCCN guidelines; another 5 (38.5\%) were in CHEK2 and changed surveillance due to published breast and colon cancer risks; and 1 did not impact management ( $M Y H$ monoallelic mutation carrier). Conclusion: NCCN criteria and corresponding single-gene testing alone, when applied to this cohort, would have failed to detect eight individuals with gene mutations resulting in medical management modifications. Additional research is needed to assess the indications and utility of MPG testing.

\section{Risk Stratification of Women at Intermediate or High Risk of Breast Cancer: Developing a Consensus Framework for Screening and Prevention}

\author{
J. Gagnon ${ }^{1}$, E. Lévesque ${ }^{1}$, B. M. Knoppers ${ }^{1}$, B. Lespérance ${ }^{2}$, J. Simard ${ }^{3}$ \\ 1. Gentre of Genomics and Policy, McGill University \\ 2. Sacré-Coeur Hospital \\ 3. Department of Molecular Medicine, Laval University
}

Introduction: New genetic screening tests for common and low frequency genetics variants are currently under development. These tests, along with improved risk prediction tools will make the identification of young women at intermediate or high risk of breast cancer widely available. However, there are currently no guidelines or tools for the counseling and management of these women. Purpose: Build and test the framework for experts consensusbased guidelines for breast cancer screening \& prevention in intermediate/high risk women. Methods: We developed draft communication tools (risk stratification curves and decisional tree) as well as 69 evidence-based Q\&A by reviewing international guidelines and literature. Both were validated with an interdisciplinary expert panel. Topics covered referral to genetics or breast clinic, radiology, breast exam, risk reduction measures, and preventive lifestyle choices. An extended expert panel attended a 2 day meeting to answer questions (with an audience-response system) and to debate so as to reach consensus. Results: The extended expert panel included 44 experts from 11 specialties, including geneticists and genetic counselors. A consensus ( $>=$ $80 \%$ voter agreement) was obtained on 32 questions. Consensus was easily reached on the topics of: referral to specialty clinics, risk reduction measures and preventive lifestyle choices. There was however more debate on the issues of imaging technology (choice \& timing) and breast examination. Satisfaction with the process was very high $(97.4 \%)$. The risk stratification curves and decisional tree were well understood and accepted tools. Conclusion: An interdisciplinary panel of experts is an adequate framework to achieve consensus on the broad range of issues inherent in comprehensive guidelines for counseling and management for breast cancer risk. Background "evidentiary" materials of high quality as well as open debate can serve to reach consensus on sensitive issues. End-users should be involved at every step in order to optimize guidelines implementation.

\section{Atypical Phenotypes of FAP and MAP Patients Ascertained through Multi-Gene Hereditary Cancer Panels}

\section{J. Guiltinan ${ }^{1}$, L. Littrell ${ }^{1}$, M. Umali ${ }^{1}$, H. LaDuca ${ }^{1}$}

\section{Ambry Genetics}

Familial adenomatous polyposis (FAP) and MUTYH-associated polyposis (MAP) can often be unambiguous syndromes to diagnose clinically. The presence of multiple adenomatous polyps generally leads a clinician to order testing specifically for the APC or MUTYH genes. However, for patients in which the clinical phenotype does not represent any classical hereditary cancer syndrome, clinicians can order a multi-gene panel; thus, it is possible for patients with phenotypes not classic of FAP and MAP to present with mutations in APC or MUTYH, respectively. Do individuals with mutations in either $A P C$ or $M U T Y H$ ascertained by multi-gene testing present with atypical phenotypes? A database search of Ambry internal data was performed for all multi-gene hereditary cancer panels performed from 3/16/12 through 3/14/14. Patients confirmed to have a mutation or likely pathogenic variant (VLP) in $A P C$ or bi-allelic mutations or VLPs in $M U T Y H$ were curated. Individuals positive for the moderate penetrance $A P C$ mutation, p.I1307K, were excluded from this study. A total of 33 individuals were ascertained from the database. These patient results are consistent with a diagnosis of either FAP $(48.5 \%)$ or MAP $(51.5 \%)$. In total, $24.24 \%$ of these patients had no personal history of cancer. A total of $21.2 \%$ of patients had no personal history of polyps, which is atypical, and $71.4 \%$ had at least one small deletion or duplication, in contrast to $33.3 \%$ of the total cohort. Interestingly, 3 patients had a personal history of breast cancer in their 50s, and 1 MAP patient had ovarian cancer at 37 and no polyps. FAP and MAP patients ascertained through multi-gene hereditary cancer panels mostly present with the typical phenotype, however, one patient with MAP presented with the atypical phenotype of 
ovarian cancer and no polyps. In addition, a significant amount of FAP and MAP patients did not have a history of polyps, and these patients were found to carry small deletion/duplications at a higher frequency than the rest of the FAP/MAP cohort, possibly suggesting a phenotype/genotype correlation.

Identification of Lynch Syndrome Families with Female Reproductive Tract Cancers in the Era of Next Generation Sequencing

C. Ikard ${ }^{1}$, J. Eichmeyer ${ }^{1}$, H. Hussey ${ }^{1}$, T. Beck ${ }^{1}$

1. St. Luke's Mountain States Tumor Institute

Currently, the identification of Lynch syndrome (LS) is based on meeting specific criteria (e.g. Amsterdam I or II) which is known to pick up only $50 \%$ of LS families. With the development of high throughput technologies, such as next generation sequencing (NGS), the use of multiplex hereditary cancer panels is becoming more widely used and less expensive than single syndrome testing. Often, NGS is serving as first line testing, rather than relying only on the clinical criteria or gene by gene Sanger sequencing. Since 2012, our community based cancer center has been offering NGS, and in that time, 26 patients with an ovarian and/or uterine cancer diagnosis have elected genetic testing by a NGS hereditary cancer panel. Of these, 8 received positive or variant of uncertain significance (VUS) results in one of the LS genes (30.8\%). Only 1 of these 8 families met Amsterdam II Clinical Criteria (12.5\%). Interestingly, the proband in the family meeting clinical criteria was diagnosed with ovarian cancer, which is not included in Amsterdam II. The family who met Amsterdam II had a MLH1 mutation while the other 7 families had a mutation or VUS in MSH6. Our results suggest that the current LS clinical criteria are not adequate for identification of families with Lynch syndrome particularly for female reproductive tract cancers. Our genetics group developed internal criteria based on National Comprehensive Cancer Network and/or Medicare guidelines for when to offer next generation panels. By broadening traditional diagnostic criteria internally we identified eight LS families who might have otherwise been missed. NGS is a valuable tool, especially for families not meeting diagnostic criteria or those with reduced penetrant genes. More research is needed to develop national standards for how to use this NGS testing.

I Wish I had Known This! Impact of Age on Life Choices and Testing Satisfaction for BRCA1 and BRCA2 Mutation Carriers Who Underwent Genetic Testing by Age 25

\author{
S. King ${ }^{1}$, K. Brooks ${ }^{2}$, A. Werner-Lin ${ }^{3}$, T. Herzog ${ }^{4}$ \\ 1. University of South Carolina \\ 2. South Carolina Oncology Associates \\ 3. University of Pennsylvania \\ 4. Francis Marion University
}

Introduction: Young BRCA1/2 mutation carriers between ages of 18 and 25 are in the life stage called emerging adulthood. This is a highly transitional period marked by major life choices and instability related to career, occupation, or relationships. Minimal to no guidelines exist to guide decisionmaking related to $B R C A 1 / 2$ risks. Hypothesis: Women with a $B R C A 1 / 2$ gene mutation who received their genetic test result by age 25 would be satisfied with their decision to undergo genetic testing and with life plans made. Methods: Seventy-two BRCA1/2 mutation positive women who underwent genetic testing by age 25 were recruited via social media, using convenience and purposive sampling, to complete a survey via SurveyMonkey.com. Selection criteria included: (1) BRCA1/2 mutation carriers, (2) over age 18 at the time of the study, (3) had a genetic test result before age 26. Sixty-three met criteria and were asked 40 questions designed by the principal investigator to assess satisfaction, family planning, surveillance, and surgery needs of young BRCA carriers. Quantitative data analysis was performed using Chronbach's Alpha, Chi-square, and Spearman's Rho, while qualitative data was analyzed via Content Analysis. Results: Participants were highly satisfied with the decision to undergo genetic testing and with life choices made. To guide the counseling and care of BRCA1/2 carriers under age 25, recommendations were made based on data that included: (1) Right Reproductive Organs, (2) Risk Reducing Mastectomy, (3) Risk Figures, (4) Reproductive Options, and (5) Resource for Future. Conclusion: While participants were satisfied with both testing and life decisions made, they desired more clear, supportive, and unbiased care. The complexity of $B R C A 1 / 2$ plus the lack of guidelines for $B R C A 1 / 2$ positive emerging adults led us to develop counseling recommendations. Incorporating these recommendations is key to achieving full autonomy and unbiased decision facilitation, which could be tailored to other cancer susceptibility syndromes.

\title{
Returning Hereditary Cancer Panel Results to Patients is Clinically Feasible and Appreciated by the Patients
}

\author{
K. Kingham ${ }^{1}$, N. Chun ${ }^{1}$, M. Rabideau ${ }^{2}$, A. Kurian ${ }^{1}$, S. Lincoln ${ }^{2}$, J. Ford ${ }^{1}$
}

1. Stanford University

2. Invitae

As broad multi-gene panels enter clinical use, unique genetic counseling challenges arise, particularly in cases where a result is unexpected or for which there are no established clinical guidelines. Recently we described results from panel testing for hereditary breast and ovarian cancer, where 14 of 141 $B R C A 1 / 2$ negative patients had potentially actionable findings in other genes. Here we describe our experience in counseling these individuals, focusing on informed consent, communication of risk, clinical management, and psychosocial issues. In this study a 42-gene panel was run under a research protocol 1-10 years after each patient's clinical $B R C A 1 / 2$ test. Return of research results is appropriately debated, although this new information was felt to be of significant value to the patients. Stanford's Institutional Review Board approved a protocol under which patients could be re-contacted and reconsented. Patients were offered clinical confirmation of the research finding in a CLIA-approved lab. Ten of the 14 patients and the children of one deceased patient were contacted. All expressed interest in the new findings and returned to our clinic to review results. Positive results in $A T M, B L M$, $C D H 1, M U T Y H, M L H 1, N B N$, PRSS1 or SLX4 were returned with recommendations for additional screening and in one case, prophylactic surgery. Reproductive implications for recessive disorders were discussed. All expressed gratitude for the additional information. Although one participant 
initially expressed significant anxiety to the unexpected result, she expressed appreciation following two additional genetic counseling sessions. We conclude that it is possible to ethically and successfully return broad panel results, to patients not initially consented for this information. For some patients, pre-test anticipatory guidance would be beneficial. We have expanded the study cohort with another 250 patients showing a similar rate of nonBRCA events (up to $10 \%$ ) and we continue to refine these counseling practices for our patients.

APC Mutations in Children with Hepatoblastoma: Evidence for Genetic Evaluation for Familial Adenomatous Polyposis as Standard of Care

\author{
S. Knapke ${ }^{1}$, E. Mundt ${ }^{1}$, G. Tiao ${ }^{1}$, J. Geller ${ }^{1}$ \\ 1. Cincinnati Children's Hospital Medical Center
}

Introduction: Hepatoblastoma is most common hepatic cancer in childhood and is generally considered to be sporadic. In some cases, it is a rare but welldescribed feature of familial adenomatous polyposis syndrome (FAP). There is limited and conflicting data in the medical literature regarding the frequency of germline $A P C$ mutations in children who are diagnosed with hepatoblastoma. Methods and Results: In a single pediatric academic medical center, 25 individuals with a diagnosis or history of hepatoblastoma were prospectively tested for $A P C$ mutations. Four of $25(16 \%)$ were positive for a deleterious $A P C$ mutation and one patient was found to have an $A P C$ variant of uncertain clinical significance. Both children with a family history consistent with clinical FAP tested positive for $A P C$ mutations, while 2/23 (8.7\%) remaining patients with unremarkable family histories tested positive for deleterious germline $A P C$ mutations. Conclusions: Germline $A P C$ mutations are not uncommon in individuals with hepatoblastoma. While collecting a thorough family history is a valuable screening tool, due to a significant de novo mutation, cases of FAP would be missed by limiting testing to only those children with hepatoblastoma with suggestive family histories. The clinical impact of undiagnosed and unmanaged FAP, with substantial risks for development of colon cancer and risk of cancer-related mortality among other comorbidities, cannot be underestimated. Data suggest that germline testing for $A P C$ mutations should be offered in all cases of children with hepatoblastoma.

Features of Hereditary Breast and Ovarian Cancer in a Lynch Syndrome Cohort Ascertained through Multi-Gene Panel Testing

H. LaDuca ${ }^{1}$, C. Horton ${ }^{1}$, T. Pesaran', P. Summerour ${ }^{1}$, C. Espenschied ${ }^{1}$, C. Mason ${ }^{1}$

\title{
1. Ambry Genetics
}

Numerous studies evaluating the association between Lynch syndrome (LS) and breast cancer have yielded conflicting results, and the overlapping phenotypes of LS and Hereditary Breast and Ovarian Cancer syndrome (HBOC) are even less well explored. Here we assess the clinical and molecular characteristics of individuals found to harbor mismatch repair (MMR) and EPCAM mutations on multi-gene panel testing, with a focus on features of HBOC. Our sample consists of 112 patients with a MMR gene or EPCAM mutation identified by multi-gene panel testing between March 2012 and March 2014. Clinician-reported clinical histories were reviewed to determine whether criteria for LS (Amsterdam I, Amsterdam II, and/or revised Bethesda criteria) or testing criteria for HBOC were met (National Comprehensive Cancer Network HBOC testing criteria, version 1.2014). Fifty-seven percent $(n=64)$ of MMR/EPCAM mutation carriers met LS criteria. Thirty-eight percent $(n=43)$ of MMR/EPCAM mutation carriers met HBOC testing criteria, $20(17.9 \%)$ of whom met both LS and HBOC testing criteria, and $23(20.5 \%)$ of whom met only HBOC testing criteria. Amongst probands meeting LS testing criteria, mutations were identified most frequently in $M L H 1(31.3 \%, n=20)$, followed by $M S H 2(28.1 \%, n=18), M S H 6(20.3 \%, n=$ 13), PMS2 $(18.8 \%, n=12)$, and $\operatorname{EPCAM}(1.6 \%, n=1)$, whereas $M S H 6$ was the most frequently mutated gene amongst HBOC probands $(44.2 \%, n=19)$, followed by $M L H 1(23.3 \%, n=10), M S H 2(16.3 \%, n=7)$ and PMS2 $(16.3 \%, n=7)$. Breast cancer was reported in $18.8 \%(n=21)$ of probands with an MMR mutation, and ovarian cancer was reported in $14.3 \%(n=16)$ of probands. The difference in the distribution of mutations in MSH6 amongst patients meeting criteria for LS and HBOC adds to the existing knowledge of potential genotype-phenotype associations in LS. One fifth of patients with MMR mutations met criteria for HBOC but not LS, representing a significant portion of patients with potentially misleading histories and highlights the importance of including LS as a differential for families with breast and ovarian cancer.

\section{The Angelina Jolie Effect: Assessing the Impact of a Celebrity's Story on Cancer Genetic Counseling}

\section{MacCuaig ${ }^{1}$, J. Krejdovsky ${ }^{2}$, K. Bloch ${ }^{3}$, A. Pollak ${ }^{4}$, E. Cross ${ }^{1}$, T. Cross ${ }^{1}$, G. Schneider ${ }^{1}$}

\section{Brandeis University \\ 2. Beth Israel Deaconess Medical Center \\ 3. Dartmouth Hitchcock Medical Center \\ 4. Patient Advocate}

On May 14, 2013 The New York Times printed an op-ed piece by actress Angelina Jolie entitled, "My Medical Choice" in which she disclosed that she carries a BRCA1 gene mutation. Mutations in BRCA1 increase a woman's lifetime risk of breast and ovarian cancer, to as high as $87 \%$ and $50 \%$, respectively, Ms. Jolie elected to have a prophylactic double mastectomy and her public disclosure of this decision put a spotlight on hereditary cancer and genetic testing. We sought to assess the impact of her story, now often referred to as the "Angelina Jolie Effect", on cancer genetic counseling and patient decision-making. We distributed an anonymous, online survey via e-blast to the National Society of Genetic Counselors' listserv, recruiting individuals who provide cancer genetic counseling. We analyzed 178 completed surveys using SPSS and found that in the interim since the publication of the op-ed piece, $89 \%$ of respondents experienced increases in calls requesting appointments for risk assessment and/or genetic testing, $84 \%$ saw increases in the number of low-risk individuals requesting genetic testing, and $64 \%$ reported increases in the number of patients seen per week. A majority of genetic counselors (69\%) also felt there was an increase in the number of low-risk individuals requesting genetic testing. Additionally, respondents believed that, since the publication of The New York Times op-ed piece, there was an increased likelihood that BRCA1/2 mutation carriers would ask about and/or pursue prophylactic mastectomy/oophorectomy, though the increase was greater for mastectomy. Our study provides the first data regarding the impact of Angelina Jolie's story on cancer genetic counseling. Genetic counselors need to be prepared for the effects of celebrity stories 
on their clinical practices and patients, and take an active role in helping the media to better communicate the complex issues associated with stories like Angelina Jolie's.

Hereditary Breast and Ovarian Cancer: Implementation of Genetic Counseling Within the High Risk Program of the Breast Center at Clínica Alemana of Santiago, Chile

\section{S. Beatriz Margarit ${ }^{1}$}

\section{Clínica Alemana, Universidad del Desarrollo}

Breast cancer is one of the most common cancers in women, 5-10\% are hereditary and of these, $30-50 \%$ are due to mutations in high risk susceptibility genes; BRCA 1 and BRCA 2. In order to understand the implications of genetic predisposition studies and obtain appropriate preventive measures and medical management options many patients and families would benefit from referral to genetic counseling. However, in Chile as well as in most South American countries, genetic counseling is not integrated in high-risk cancer programs. Since May 2013, the high-risk breast cancer center of the German Clinic of Santiago (Clínica Alemana de Santiago) has developed a high risk cancer genetics program integrated by a supervisor physician, a nurse coordinator and U.S.A. trained genetic counselor. The main goals of the program are: 1. Identification of hereditary high-risk families based on personal and family history; 2 . Provision of non-directive genetic counseling and psychosocial support; 3 . Education of hereditary and cancer risks; 4 . Discussion of options of molecular genetic studies; 5. Information and options of appropriate monitoring and risk management. Since the inception of the program in May 2013 until the March 2014, 136 referrals have been made with the assistance of 65 patients compared with a total of 38 patients who obtained genetic counseling in Preventive Medicine from August 2009 to April 2013. Conclusion: Incorporating a genetic counseling program within a high risk cancer interdisciplinary team allows better access to genetic counseling, improves management and appropriate monitoring of patients and healthy relatives with hereditary risk.

\section{Hereditary Cancer Panels: Clinical Utilization, Testing Strategies and Genetic Counselors' Knowledge of Technology}

\section{McKenna ${ }^{1}$, Z. Ali-Khan Catts ${ }^{2}$, S. J. Robbins ${ }^{3}$, L. Kessler ${ }^{1}$}

1. Genetic Counseling Program, Arcadia University

2. Christiana Care Health Systems

3. Arcadia University

Multiplex test panels using Next-Generation sequencing (NGS) technologies were introduced into the cancer genetics setting in 2012. Multiplex testing allowed for several genes associated with hereditary cancer syndromes to be sequenced at the same time in a cost effective manner. This development has the potential to reduce patient anxiety during the testing process and to decrease wait times for results. However, some health care professionals may be hesitant to order multiplex testing, as cancer risks and management recommendations are not well defined for some genes on the test panels. Additionally, there are no existing guidelines for when multiplex testing should be offered. The purpose of this study was to ascertain genetic counselors' knowledge about NGS technologies, and to determine the clinical utilization and testing strategies of multiplex test panels. Surveys were distributed at a national meeting and completed by 125 practicing genetic counselors. Data was analyzed using SPSS software with a series of two-tailed t-tests and chi-square analysis. Results showed that more genetic counselors are ordering multiplex test panels for breast cancer compared to colon panels. Genetic counselors with greater years of experience were more likely to order multiplex testing compared with less experienced counselors. Knowledge of both Sanger sequencing and NGS was not correlated with years of experience as a genetic counselor. Additionally, the average knowledge score for NGS technologies was 12 percentage points higher than the average knowledge score for Sanger sequencing technology which was contradictory to the original hypothesis. This research also demonstrated a lack of consensus among genetic counselors for managing challenging scenarios that can occur when ordering multiplex test panels reflecting the need for updated clinical guidelines.

\section{A Year of Unexpected Results: How the New Panels and a Non-Geneticist Diagnosed Three Patients with Hereditary Cancer Syndromes that a Genetic Counselor Would Have Missed}

\section{S. Morrill-Cornelius ${ }^{1}$}

\section{Danbury Hospital}

Introduction: Over the past year, we have had three cases where patients were diagnosed with a cancer syndrome that would have been missed using standard genetic testing algorithms based on National Comprehensive Cancer Network criteria. Case Presentations: 1. A 44-year-old male with colorectal cancer and 14-15 colorectal polyps was referred for genetic counseling. Family history was remarkable for pancreatic cancer in his father and gastrointestinal cancer in a distant maternal cousin. Colonext revealed a mutation in the APC gene, leading to a diagnosis of attenuated familial adenomatours polyposis. In the weeks after his diagnosis we received information from the laboratory that they had been validating their breast cancer gene panel when this man's testing was completed, and his data had been inadvertently unmasked. They indicated that there was a potentially concerning mutation and after discussion with the patient, they went on to confirm that he also has a mutation in BRCA2. 2. An 83-year-old female with uterine cancer at 50 and a family history of breast and ovarian cancer was seen for genetic counseling. BRCA analysis was normal for her daughter, who had triple negative breast cancer at 57. We suspected a diagnosis of Lynch syndrome. Genetic analysis of the 24 genes associated with colorectal and breast cancer identified a mutation in BRCA2, proving her daughter is a phenocopy. 3. A 43-year-old male was diagnosed outside our institution with rectal cancer and his gastroenterologist sent analysis of the Lynch syndrome genes. His resection specimen had normal Lynch syndrome screening by immunohistochemistry and his family history unremarkable. While we would not have ordered the costly Lynch syndrome genetic testing, it identified a mutation in the PMS2 gene, indicating that this patient has Lynch syndrome. Conclusion: Our sampling bias over the last years has kept the study 
population enriched for the highest risk patients and missed others with a more moderate phenotype. As we have increased access to analyze the genome, the phenotype of syndromes that were previously thought very high risk will change and this deserves further study.

\title{
Cancer Genetics Knowledge in Orthodox Jewish Women with and without a Family History of Cancer in the Greater Detroit Area
}

\author{
T. Niemchak ${ }^{1}$, M. Fisher ${ }^{2}$, M. Simon ${ }^{1,2}$, R. Tkatch ${ }^{1,2}$, T. Albrecht ${ }^{1,2}$
}

1. Karmanos Cancer Institute

2. Wayne State University

Knowledge about cancer risk factors including the effect of family history on cancer risk is influenced by whether or not there is a family history of cancer. There is a high prevalence of hereditary breast and ovarian cancer syndrome and low utilization of cancer genetics services in the Orthodox Jewish community. In order to better understand barriers to cancer genetic counseling, the relationship between family cancer history and knowledge of cancer risk factors was evaluated in that community. A 16 page randomly mailed survey of cancer risk factors, knowledge, and attitudes towards cancer screening was administered to women in the Orthodox Jewish community of Metropolitan Detroit (response rate 260/450; $57.8 \%$ ). Responses to 23 items from a validated breast cancer genetic counseling knowledge questionnaire were analyzed. Women without a personal history of cancer $(88 \%$ of respondents) were stratified by history of cancer in a first degree relative ( $n=107$-yes; $126-$-no). Knowledge scores were computed by the sum of total correct responses; summary scores were compared using independent sample t-tests. The mean overall knowledge score was 8.50 (SD 5.5); the score for those with family cancer history was 9.4 (SD 5.6; $40.8 \%$ correct) and 7.6 (SD 5.3; $33.0 \%$ correct) for those without family history ( $p=0.013$ ). The majority $(73.8 \%)$ knew that a positive genetic test results in an increased risk for breast cancer, however few $(3.5 \%)$ knew their risk of carrying a hereditary mutation if their sister was a carrier. For the selected items, women with a family history of cancer were less likely to select "I don't know," and more likely to answer correctly. A family history of cancer was associated with increased knowledge of cancer risk factors but further education is needed. Future directions include analyzing screening intentions and behaviors to determine if increased knowledge is associated with increased screening.

\section{Do Personal or Family History of Renal Cell Carcinoma Predict the Likelihood of an Inherited Cancer Syndrome: Preliminary Results from a Multi Gene Hereditary Renal Cancer Test \\ L. $\operatorname{Panos}^{1}$, M. Pritzlaff ${ }^{1}$ \\ 1. Ambry Genetics}

Purpose: Genetic testing for hereditary renal cell carcinoma (RCC) is typically considered in RCC cases with syndrome-specific findings and/or histology. However, nonspecific clinical presentation can pose a challenge to this testing approach. This study describes the utilization of multi-gene testing for RCC in the clinical setting. Methods: Clinical histories obtained from ordering clinicians of 126 individuals undergoing RCC multi-gene testing between August 2013 and March 2014 were assessed. Sequencing and deletion/duplication analyses of FH, FLCN, MET, MITF, MLH1, MSH2, MSH6, PMS2, PTEN, SDHA, SDHB, SDHC, SDHD, TP53, TSC1, TSC2, VHL, and duplication/deletion analysis of EPCAM were performed. Results: Of 126 individuals, $86 \%$ had a personal history of RCC. A mutation was identified in 14 (11.1\%) probands, including 4 mutations in $F L C N$ and $F H, 2$ in $P M S 2$, and 1 in MSH2, MITF, TSC1, and TSC2. Eleven positive probands had a history of RCC. Histologies varied, including papillary (3), clear cell (2), chromophobe (1), mixed (1), and unspecified (4). The mean age at RCC diagnosis was 41 years in carriers and 45 in non-carriers. Classic features consistent with the molecular result were reported in $36 \%$ of carriers, including individuals with mutations in FLCN. FH, TSC1 and TSC2. No mutations were identified in 13 families with 3 or more individuals with RCC. Of 105 families with 2 or fewer individuals with RCC, 11 (10.5\%) carried a mutation. Conclusion: Age at RCC diagnosis did not appear predictive of positive results in this cohort, nor did the number of individuals with RCC in a family. Thirty six percent of mutations identified were in individuals with classic hereditary RCC syndrome features for the correlated gene, suggesting that single-gene testing is preferable in patients with classic presentation. However, multi-gene tests have utility for patients without classic presentation. These findings warrant further investigation in a larger cohort to determine the best use of multi-gene testing for hereditary RCC.

\section{Before It's Too Late: Broad Hereditary Cancer Panel Testing at the End of Life}

\author{
M. Rabideau ${ }^{1}$, B. Vikstrom² ${ }^{2}$ K. Vikstrom ${ }^{1}$, K. Nykamp ${ }^{1}$, S. Topper ${ }^{1}$, M. Powers ${ }^{1}$
}

\section{Invitae Corporation}

2. Northbay Medical Center

Recent publications discuss targeted testing for patients dying of ovarian cancer. Less is known about genetic testing at the end of life for patients with complex personal and family cancer histories that do not fit guidelines for genetic testing. We present the case of a patient who had a 29-gene hereditary cancer panel submitted shortly before his death, which detected a pathogenic PALB2 germline mutation. The patient was a 75-year-old man diagnosed at age 58 with prostate cancer who presented with pancreatic cancer at age 74 . His family history included a son diagnosed with an ampullary carcinoma at the age of 43 , a mother and maternal aunt who died of ovarian cancer in their 50s, and a maternal grandmother with breast cancer at age 55 . The patient did not meet Medicare criteria for BRCA1/BRCA2 testing. The patient underwent a 29-gene hereditary cancer panel that included BRCA1 and BRCA2. Within days the patient rapidly declined and expired before the results were available. The patient was found to have a pathogenic $P A L B 2$ c.1671 1676delinsGC (Phe557Leufs*3) mutation, and this result was disclosed to his wife and daughter. Although this was a difficult time for the family, testing provided relevant cancer risk information for the patient's daughter and grandchildren. Broader genetic testing can be helpful for the families of terminally ill cancer patients, because it improves risk calculation for surviving family members. Even if the results are in a lower penetrance gene, for which there are no management guidelines today, new guidelines and improved risk calculation models are likely to available in the future. If variants of uncertain significance are identified, they can be followed over time. We discuss our experience with this family, and talk about the 
importance of DNA banking for terminally ill patients. We discuss the counseling challenges of working with the family of a deceased patient. We also delve into the issues of insurance coverage and test reimbursement for patients after they have passed away.

Detection of Pathogenic Mutations in Moderate Penetrance Breast Cancer Genes Significantly Increases the Number of Patients Identified as Candidates for Increased Screening

\author{
E. Rosenthal ${ }^{1}$, H. McCoy ${ }^{1}$, K. Moyes ${ }^{1}$, B. Evans ${ }^{1}$, R. Wenstrup ${ }^{1}$
}

1. Myriad Genetic Laboratories, Inc.

Hypothesis/Purpose: We sought to establish the clinical utility of multi-gene hereditary cancer panels for the identification of patients who may benefit from interventions to reduce cancer risk, focusing on 3 genes in which pathogenic mutations are estimated to carry a $>20 \%$ lifetime risk for breast cancer: CHEK2, PALB2 and ATM. This level of risk meets professional society recommendations for initiating breast screening at younger ages and the use of MRI in addition to mammography. We determined the proportion of women identified as candidates for modified screening through genetic testing who would not have been identified with family history. Methods: We used the Claus tables to evaluate the reported family histories of women in whom pathogenic mutations were found in CHEK2, PALB2 and ATM through clinical testing with a 25-gene hereditary cancer panel. We determined the proportion of these women who would have been identified as having a $>20 \%$ lifetime breast cancer risk based on family history. Results: Among 9,201 patients tested between 09/04/2013 and 04/17/2014, 174 female patients were identified with a single mutation in either: $C H E K 2(n=71), P A L B 2(n=39)$ and ATM ( $n=64)$. Three patients carried mutations in 2 of the genes. Excluding 5 women who also carried a mutation in BRCA1 or BRCA2, only 15 $(8.7 \%)$ of the remaining 172 women reached the $>20 \%$ threshold for lifetime breast cancer risk using the Claus tables. By comparison, among this same group of 9,201 tested individuals, 275 had a pathogenic mutation in BRCA1 or BRCA2. Therefore, inclusion of the 3 moderate penetrance genes improved the sensitivity of the testing, as defined by the identification of a genetic finding associated with an established breast cancer screening recommendation, by $62.5 \%$. Conclusions: Clinical testing including the moderate penetrance genes $C H E K 2, P A L B 2$ and $A T M$ significantly increases the likelihood of identifying women who can benefit from modified medical management strategies that would not have been applied based on family history alone.

\title{
Multiple Lessons Learned from a Single Cancer Genetics Referral: Unusual Presentation of Monoallelic Mismatch Repair Deficiency
}

\section{K. Schneider ${ }^{1}$, N. Foreman ${ }^{1}$ \\ 1. University of Colorado Denver}

A single genetic counseling case illustrates the challenges in evaluating atypical clinical presentations of Lynch syndrome, appropriateness of certain specimens for analysis following allogeneic stem cell transplant, and importance of comprehensive genetic counseling that includes good communication throughout the genetic testing process. The diagnostic evaluation of hereditary cancer syndromes in this case was complicated by an unusual clinical presentation. Proband presented with bifrontal anaplastic oligodendroglioma at age 20. Family history includes the following diagnoses: father with colorectal cancer at age 61, paternal aunt with cervical carcinoma and ovarian clear cell adenocarcinoma at age 42, and sister with adrenocortical carcinoma at age 24. History was most suggestive of biallelic mismatch repair deficiency; Li-Fraumeni syndrome was also considered. The American founder mutation in MSH2 (deletion of exons 1-6) was detected in each of the affected individuals. No other mutations were detected in TP53 or MSH2 for the proband or his sister, suggesting that this family had an atypical presentation of monoallelic mismatch repair deficiency. This case was further complicated by the proband's history of stem cell transplant at age 9 for Ph positive acute leukemia with his brother as donor. Multiple genetic tests were performed on the proband's peripheral blood before realizing that the results represented his brother's genetic status. The tests were then repeated on cultured skin fibroblasts and revealed the same results. Initial presentation to genetic counseling from the care team was via e-mail requesting assistance with TP53 and APC test ordering. Abbreviated genetic counseling was held for informed consent and pedigree assessment, revealing the paternal aunt's pending evaluation for Lynch syndrome. Comprehensive genetic counseling with the proband, mother, and both siblings, directed the family to more appropriate analyses and provided a structure for results disclosure, easing the handling of the earlier than anticipated results for the brother.

\section{Hereditary Breast Cancer Testing: When Results Are Not Straightforward}

\author{
L. Servais ${ }^{1}$, T. Donenberg ${ }^{2}$, D. Bonner ${ }^{3}$, A. Monteiro $^{3}$, M. Akbari ${ }^{4}$, S. Vadaparampil $^{3}$, T. Pal ${ }^{3}$ \\ 1. Care Core National \\ 2. University of Miami \\ 3. Moffitt Cancer Center \\ 4. University of Toronto
}

Introduction: Since the fall of the BRCA patent in June 2013, several commercial laboratories have entered the market to offer the test, which has led to variations in interpretation of test results across laboratories. Through a population-based study to evaluate BRCA mutations in young Black women in Florida, a 36 year old participant with a personal history of three primary breast cancers was recruited in 2012. Clinical BRCA testing in 2004 identified a BRCA1 (c. 110C > G, p.Thr37Arg) missense variant of uncertain significance (VUS) and subsequent large rearrangement testing was negative. Methods: In Spring 2014, the original testing laboratory was contacted to request updated results. Additionally, two academic researchers and four commercial labs that offer clinical BRCA testing were contacted to supplement this information. Results: Data shared by the original testing lab continued to classify this $B R C A 1$ alteration as a VUS without providing information regarding how this conclusion had been reached. The academic researchers indicated this variant was highly suspicious for pathogenicity and classified it as IARC Class 5 . Responses across the four commercial laboratories (none of whom had seen it before) varied as follows: Lab A: Classify as VUS, but closer to pathogenic than benign. Lab B: Apologized that they could not be of more help. Lab C: Classify as suspected deleterious primarily based on functional studies. Lab D: Classify as VUS, but indicated that functional studies suggest 
potential pathogenicity. Discussion: This case highlights variations across laboratories in both: 1) providing information to clinicians on VUS results and 2) the interpretation of VUS. Furthermore, it raises several genetic counseling issues - should we be asking/pressuring labs to "show their work" regarding how they are classifying VUS, should we be seeking "second opinions" on VUS interpretations, what happens when the opinions conflict and how do all of these issues affect the management of the patient and her family?

Newly-Described Genes on the GeneDx Comprehensive Cancer Panel: Pathogenic/Likely Pathogenic Variants Detected in Patients Satisfying National Comprehensive Cancer Network Testing Guidelines

S. Solomon ${ }^{1}$, K. Hruska ${ }^{1}$, D. Pineda-Alvarez ${ }^{1}$, M. Baxter, PhD ${ }^{1}$, P. Murphy ${ }^{1}$, M. Cremona ${ }^{1}$, J. Booker ${ }^{1}$, L. Susswein ${ }^{1}$, L. Andolina ${ }^{1}$, K. J. Vogel Postula $^{1}$, R. Klein ${ }^{2}$

1. GeneDx

2. BioReference Laboratories

Introduction: Inherited cancer panel testing via Next Generation Sequencing (NGS) technology has expanded options for diagnosing hereditary cancer syndromes in at-risk patients. NGS inherited cancer panels analyze well-described, highly penetrant genes for which the National Comprehensive Cancer Network (NCCN) has established Clinical Practice Guidelines in Oncology (testing guidelines). These cancer panels also evaluate newly-described cancer genes which, by virtue of less data on inherited cancer risk, have no testing guidelines. The GeneDx Comprehensive Cancer Panel analyzes 29 genes including 12 newly-described genes associated with cancer: ATM, AXIN2, BARD1, BMPR1A, BRIP1, CHEK2, FANCC, NBN, PALB2, RAD51C, $R A D 51 D$, and $X R C C 2$. Purpose: Our aim was to identify the frequency of patients who harbored a Pathogenic or Likely Pathogenic Variant (PV/LPV) in a newly-described gene detected on the GeneDx Comprehensive Cancer Panel who also met current testing guidelines. Methods: Patients with PV/LPVs in a newly-described gene on the GeneDx Comprehensive Cancer Panel were queried. Pedigree analysis was performed to distinguish which patients met testing guidelines. Results: Over 8 months, 824 GeneDx Comprehensive Cancer Panels were ordered. At least one PV/LPV was detected in 79/824 $(9.6 \%)$ of patients. Of these patients, $40 / 79(50 \%)$ had at least one PV/LPV in a newly-described cancer predisposition gene. Pedigree analyses showed 29/40 (72 \%) of patients with PV/EPVs in these genes met testing guidelines. Conclusions: Half of PV/LPVs detected on the GeneDx Comprehensive Cancer Panel were found in newly-described cancer predisposition genes. Of patients with a PV/LPV, almost three-quarters of variants were found in a newly cancer-associated gene, which would otherwise have been undetected using NCCN testing guidelines for single gene analyses. NGS inherited cancer panels provide access to additional genetic information to patients and families for personalized cancer risk assessment and medical management.

\title{
Examining Gastrointestinal Stromal Tumor Patients' Understanding of Tumor Mutation Analysis and Personalized Medicine
}

\author{
S. Stickevers ${ }^{1}$, M. Campion ${ }^{1}$, E. Thorpe ${ }^{2}$, E. Dalton ${ }^{3}$, \\ 1. Boston University School of Medicine \\ 3. Brigham and Women's Hospital \\ 4. Ambry Genetics
}

Somatic mutations in KIT, PDGFRA, and BRAF identified through analysis of gastrointestinal stromal tumors (GISTs) have been correlated with response to therapy, treatment dose, recurrence risk, and prognosis. While the use of tumor mutation analysis (TMA) is expanding, little is known about the understanding and attitudes of patients towards these new medical advances. Adults with GIST were identified through the GIST Support International patient group and anonymously surveyed to quantitatively assess TMA uptake, understanding, attitudes, and misinterpretations. Out of 193 respondents, $69.3 \%$ were female and $62.2 \%$ were between the ages of 50-69 years. $75.5 \%$ received treatment in the United States while $24.5 \%$ received care internationally. $48.2 \%$ of participants discussed TMA with a health care provider and $53.1 \%$ of participants received TMA on their GIST. $44.7 \%$ reported that a health care provider initiated TMA, but $26.2 \%$ of respondents reported that they initiated the process of TMA themselves. The majority of participants, regardless of TMA uptake, were able to correctly identify benefits of TMA, including that TMA could affect treatment, provide information on prognosis and recurrence risk, and improve research for current and future GIST patients. However, many participants misattributed qualities of germline testing to TMA. Participants incorrectly reported that tumor mutation analysis could be useful in defining familial risk ( $36.2 \%$ of participants who spoke to a provider about TMA; $34.4 \%$ of participants who received TMA). Many participants reported that insurance companies could use TMA results for discriminatory purposes (14.6\% of participants who spoke to a provider about TMA; $16.0 \%$ of participants who received TMA). The misinterpretations identified in this study may be due to the manner in which test information is disseminated. Genetic counselors may be beneficial resources to patients undergoing tumor mutation analysis, as they are providers skilled in translating complex genomic information and addressing patient misconceptions.

\section{Unexpected $R A D 51 C$ and $R A D 51 D$ Findings in Breast Cancer Only Families}

A. Stuenkel ${ }^{1}$, S. Tandy ${ }^{1}$, M. Umali ${ }^{1}$, B. Tippin Davis ${ }^{1}$, H. Laduca ${ }^{1}$

\section{Ambry Genetics}

Mutations in the RAD51C and RAD51D (RAD51C/D) genes have been recently described in hereditary breast and ovarian cancer families and associated with significantly increased ovarian cancer risk; however, the correlation between these genes and breast cancer susceptibility remains controversial. In this study, we aim to clarify the phenotypic spectra associated with $R A D 51 C / D$ through retrospective review of molecular and clinical data from patients referred for diagnostic testing. Data from multi-gene hereditary cancer tests that included analyses of RAD51C from March 2012 and RAD51D from December 2013 through May 2014 were reviewed. Only alterations resulting in premature truncation or disruption of the canonical splice sequence were considered mutations. For individuals found to carry $R A D 51 C / D$ mutations, clinical information was obtained from clinician-reported history on test requisition forms with additional clinical data collection via follow-up clinician phone calls. Data from a total of 6,111 patients were included in the final 
analysis. In total, 26 individuals were found to have a mutation in either RAD51C or RAD51D. RAD51C mutations were identified in 18/6111 individuals $(0.3 \%)$, while $8 / 3681$ individuals $(0.2 \%)$ harbored a mutation in $R A D 51 D$. Reported clinical histories for $54 \%(14 / 26)$ families included both breast and ovarian cancer diagnoses, while 4 (15\%) of families included ovarian cancer only. Notably, 8 (31\%) mutation-positive cases (4 RAD51C, 4 RAD51D) did not have any reported personal or family history of ovarian cancer (breast-only families). Ongoing familial mutation testing efforts in these breastonly kindreds have yet to yield any informative co-segregation data. Contrary to most published data, our results suggest that $R A D 51 C / D$ mutations are detected in familial breast cancer kindreds, even in the absence of ovarian cancer. Continued research is needed to further clarify the phenotypic variability and penetrance associated with $R A D 51 C / D$ as well as the percentage of hereditary breast and/or ovarian cancer attributable to these genes.

Patients with Multiple Pathogenic Mutations Detected by Multi-Gene Panel Testing in a Lynch Syndrome Cohort

P. Summerour ${ }^{1}$, C. Mason ${ }^{1}$, T. Pesaran ${ }^{1}$, C. Espenschied ${ }^{1}$, C. Horton ${ }^{1}$, H. LaDuca ${ }^{1}$

1. Ambry Genetics

Purpose: The aim of this study was to determine the clinical and molecular characteristics of individuals in whom multi-gene panel testing resulted in identification of a pathogenic mutation in one of the mismatch repair (MMR) genes as well as an additional pathogenic mutation. Methods: Multi-gene panel tests including analysis of the MMR genes performed from March 2012 to March 2014 were reviewed. Clinical histories of individuals who tested positive for two pathogenic mutations/likely pathogenic variants, of which at least one mutation was in a MMR gene, were assessed. Results: Seven percent (8/112) of MMR mutation carriers in this cohort were identified to carry a second pathogenic mutation/like pathogenic variant. Of these eight cases: one harbored a PTEN likely pathogenic variant, two harbored ATM mutations, three harbored CHEK2 mutations, one harbored a RAD51C mutation and one harbored two MSH6 mutations, consistent with a diagnosis of Constitutional Mismatch Repair Deficiency syndrome (CMMRD). Four cases met Amsterdam I, Amsterdam II, or revised Bethesda criteria and four did not meet any of these criteria. In addition, several cases met National Comprehensive Cancer Network testing criteria (version 1.2014) for other hereditary cancer syndromes: two met criteria for $A P C$ and $M U T Y H$ associated polyposis testing and five met criteria for Hereditary Breast and Ovarian Cancer syndrome testing. Two of the CHEK2 mutation cases and the $R A D 51 C$ mutation case had a personal and/or family history of breast cancer. Of the cases that also harbored an ATM mutation, one had a personal and family history of pancreatic cancer. Conclusion: Our data demonstrate that the clinical history associated with a MMR gene mutation and an additional pathogenic mutation can be extremely variable. Our data also highlight the importance of detecting multiple pathogenic mutations in patients and conducting further research on these individuals, as this can result in more complex counseling about inheritance, co-segregation with disease phenotype, and risk management.

\title{
NF1 Mutations Detected on Multi-Gene Cancer Panel Testing in Probands with Atypical Phenotypes
}

P. Summerour ${ }^{1}$, A. J. Stuenkel ${ }^{1}$, H. LaDuca ${ }^{1}$

\section{Ambry Genetics}

Neurofibromatosis 1 (NF1) is a highly penetrant hereditary condition, with the majority of NF1 patients meeting clinical diagnostic criteria in childhood. Women with NF1 are estimated to have an approximate 4 to 8 -fold increased risk of developing breast cancer as well as an increased risk for other cancers. As such, the NF1 gene has recently been included in multi-gene cancer panels. The purpose of this study was to assess the phenotypes of individuals in our hereditary cancer panel cohort carrying a pathogenic NF1 mutation. Multi-gene hereditary cancer panels with NF1 gene alterations reported from October 2013 through April 2013 were reviewed. Retrospective test requisition form review was used to obtain clinician-reported clinical history information for individuals with pathogenic $N F 1$ mutations. Ten individuals were identified to carry a pathogenic $N F 1$ mutation. Upon review, 6 were noted to have a clinical diagnosis of NF1 on the test requisition forms. Mutations in this group included 3 missense, 2 nonsense, and 1 frameshift. The remaining 4 individuals were not noted to have a clinical diagnosis of NF1 or other NF1-related features on the test requisition forms, nor were their family members. The absence of an NF1 clinical diagnosis and NF1-related features was confirmed by a follow-up phone call to the clinicians for all 4 cases. Mutations in this group included 2 nonsense, 1 frameshift, and 1 splicing. Clinical histories of these 4 cases included three patients diagnosed with breast cancer, two $>50 \mathrm{y}$ and one $<50 \mathrm{y}$, and one unaffected patient. Of note, the unaffected patient also carried the $A P C$ I1307K moderate risk mutation and has a family history of colon cancer. Identification of pathogenic NF1 mutations in individuals with atypical phenotypes via a multi-gene panel approach may help expand upon the clinical indications currently used for NF1 testing. Further research is needed on atypical phenotypes in the NF1 population and the potential impact on clinical management for NF1 patients and their family members.

Usability of a Breast Cancer Risk Assessment Tool in a General Mammographic Screening Population: Utilization and Implications for Future Practice

\author{
M. Truelson', M. Ahrens' ${ }^{1}$, C. Torkelson ${ }^{2}$, T. Emory ${ }^{2}$, J. Kuehn-Hajder ${ }^{2}$ \\ 1. University of Minnesota Medical Center, Fairview \\ 2. University of Minnesota Physicians
}

Recent focus has been on identifying patients with hereditary breast cancer risk by using screening tools based on clinical criteria for Hereditary Breast and Ovarian Cancer Syndrome (HBOC) and validated risk assessment models. The purpose of these screening tools is to identify those at risk of a BRCA mutation and refer them to genetic counseling for further assessment and consideration of genetic testing. The ultimate goal is to appropriately apply cancer surveillance and/or surgical risk reduction options to those with proven hereditary risk in order to reduce cancer morbidity and mortality. While previously designed tools have focused on those with hereditary cancer risk, we designed a Breast Cancer Risk Assessment Tool to identify 1) Individuals at increased breast cancer risk (lifetime risk $\geq 20 \%$ ) who may benefit from breast MRI screening and 2) Individuals meeting criteria for genetic counseling referral for HBOC evaluation. The tool utilized the Gail Model and current (2011) National Comprehensive Cancer Network criteria 
to assess all individuals receiving mammograms at our institution starting in February 2012. Individuals that screened positive on the tool were referred to the breast specialist (CT), cancer genetic counselors (MA, MT), or both services within our institution. Utilizing this tool, 20.6\% (43/209) of mammogram patients screened positive for a genetic counseling referral. Only $5.9 \%(1 / 17)$ of those referred scheduled and kept a genetic counseling appointment within our system. Of those screened, $3.3 \%$ (7/209) were identified at increased breast cancer risk. None of those referred (0/4) scheduled an appointment with the breast specialist in our system. This data has significant implications for the profession of genetic counseling, as it emphasizes the importance of physician and patient education to the success of such screening tools. If cancer genetic counseling is to remain a relevant part of the risk assessment and genetic testing process, improvements need to be made to the referral and scheduling processes used in conjunction with such screening tools.

\title{
Predictors of Therapeutic and Prophylactic Mastectomy in Breast Cancer Patients
}

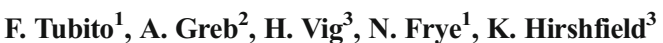

1. Long Island University

2. Sarah Lawrence College

3. Rutgers Cancer Institute of New Jersey

Background. Trends in therapeutic and prophylactic mastectomies for breast cancer are increasing despite National Comprehensive Cancer Network guidelines for equivalency in outcomes with breast conserving surgery for most women in the former and for consideration of the latter only in women with a genetic susceptibility mutation. This study was to determine clinicopathologic and demographic factors correlating with frequency of these surgeries. Methods. A randomly-selected subgroup of patients with biopsy-proven breast cancer $(n=823)$ were consented for participation from 2004 to 2010. Therapeutic and prophylactic surgeries were at the discretion of treating physicians. BRCA1/2 status was verified by genetic testing. Clinical information was evaluated for association with type of surgical procedure(s) performed. Logistic regression analyses controlling for all evaluated factors were performed. Results. Patients 39 years of age and younger at diagnoses were more likely to have therapeutic mastectomy and/or prophylactic mastectomy $(p=0.018, p=0.002)$. Patients with higher stage disease at diagnosis were more likely to undergo therapeutic mastectomy $(p<0.001)$. BRCA mutation carriers were 5.6 times more likely to have prophylactic mastectomy as compared to those without mutations $(p<0.001)$. Those with BRCA variants of unknown significance were more likely than non-carriers but less likely than carriers of deleterious mutation to undergo prophylactic mastectomy $(p<0.001)$. Family history of breast cancer in first-degree and/or second-degree relatives did not impact choice of surgical procedure in nonBRCA or BRCA-related cases. Conclusions. Age at diagnosis of breast cancer is not an indication for prophylactic or therapeutic mastectomy based on professional guidelines, but these surgeries occur at higher frequencies in this population. These results highlight factors associated with surgical decisions though the rationale for these decisions warrant further study.

\section{Low Risk of TP53 and CDH1 Secondary Findings on Inherited Cancer Panels}

\author{
K. J. Vogel ${ }^{1}$, R. T. Klein ${ }^{1}$, L. Susswein ${ }^{1}$, P. D. Murphy ${ }^{1}$, J. Booker ${ }^{1}$, M. L. Cremona ${ }^{1}$, L. Andolina ${ }^{1}$, S. Solomon ${ }^{1}$, W. Berkofsky-Fessler ${ }^{1}$, \\ J. Villar ${ }^{1}$, E. S. Rinella ${ }^{1}$, K. S. Hruska ${ }^{1}$
}

1. GeneDx

Background: Inherited cancer panels are an attractive option with the opportunity for improved detection rate and cost effectiveness, particularly given overlapping syndrome phenotypes and genetic heterogeneity. While some providers may be comfortable testing for well-described syndromes via a cancer panel, others express concern regarding risk for secondary findings, particularly in TP53 and CDH1. While management guidelines exist, a diagnosis of Li Fraumeni syndrome can be difficult given high lifetime risks, broad tumor spectrum, and limits to surveillance. Similarly, a diagnosis of hereditary diffuse gastric cancer can be challenging as aggressive management (e.g. prophylactic gastrectomy) is recommended. Additional difficulty arises when a patient's family history is not consistent with the classic phenotype. Our aim is to determine the frequency of secondary TP53 and CDHI pathogenic variants on cancer panel testing. Methods: We reviewed the provided histories of all patients who tested positive for a TP53 or CDH1 pathogenic variant on a panel to determine those who met testing/diagnostic criteria for the associated syndrome. Results: Eight of 3,914 (0.2\%) patients undergoing a panel containing TP53 tested positive for a TP53 pathogenic variant, of whom $5(62.5 \%)$ met Classic, Birch, or Chompret criteria, leading to a secondary finding rate of $0.08 \%$. Two of 3,442 (0.06\%) patients undergoing a panel containing $C D H 1$ tested positive for a $C D H 1$ pathogenic variant, neither of whom met International Gastric Cancer Linkage Consortium criteria, leading to a secondary finding rate of $0.06 \%$. Discussion: While a significant percentage of patients who tested positive for TP53 and $C D H 1$ pathogenic variants did not meet clinical criteria for the associated syndrome, the overall risk of detecting a secondary pathogenic variant is very low. The risk of secondary findings in TP53 and CDH1 is an important component of pre-test counseling for panels; however, these data allow providers to reassure patients that the risk of such findings appears to be minimal.

\section{Counseling}

“All in the Family:" Barriers and Motivators to the Use of Family History Questionnaires

S. Armel ${ }^{1}$, J. McCuaig ${ }^{2}$, N. Gojska², R. Demsky², M. Maganti ${ }^{2}$, J. Murphy' ${ }^{2}$, B. Rosen ${ }^{2}$

1. Princess Margaret Cancer Center

2. University Health Network

Data has demonstrated that family history questionnaires (FHQs) are an invaluable tool for assessing familial cancer risk and triaging patients for genetic counseling services. Despite their benefits, return rates of mailed FHQs from newly referred patients remains low, suggesting potential barriers to their use. To investigate this, a total of 461 participants, 239 who completed the FHQ (responders) and 222 who did not (non-responders), were surveyed at a 
subsequent appointment regarding potential barriers and motivators to using the FHQ. Continuous variables were analyzed using student's T-test. Categorical variables were analyzed using Chi-Square and Fisher's exact. SAS v9.2 was used for all statistical analysis. When mailed an appointment notice, $67 \%$ of non-responders attended their genetic counseling appointment. Further, $30 \%$ completed their FHQ and were subsequently treated as responders for analysis. With respective rates of $51 \%$ and $56 \%$, there was no significant difference in the proportion of responders and non-responders who reported difficulty in completing the FHQ. Both responders and non-responders reported family history factors (large family size, lack of contact with relatives, and lack of knowledge) rather than psychological factors or issues related to questionnaire design as major variables confounding completion of the FHQ. Additionally, responders were significantly more likely to state that their physician had referred them $(<0.001)$, that their physician had discussed the reason for their appointment $(<0.001)$, and that they understood the purpose of the appointment $(p=0.02)$. Overall, $68 \%$ of participants preferred providing their family history by FHQ rather than by phone or in person. These findings suggest that barriers associated with the use of FHQs are not inherent to its design, that prompts can serve to bolster completion of FHQs, and that guidance from referring physicians plays a significant role in motivating patients to complete FHQs and ultimately attend genetic counseling appointments.

Parents' Dreams for their Young Adults with Down Syndrome: What Resources Are Needed to Achieve Them?

J. Baker ${ }^{1}$, C. Brasington ${ }^{2}$, V. Vincent ${ }^{1}$, M. Will ${ }^{3}$

1. The University of South Carolina School of Medicine

2. Levine Children's Center, Carolinas HealthCare System

3. The Collaboration to Promote Self-Determination

Introduction: This study sought to identify the goals parents have for their young adult sons and daughters with Down syndrome, the factors that help to achieve those goals, and parents' perceived barriers to successful attainment of those objectives. While many supports are readily available to youths with Down syndrome in primary and secondary school, many of these supports disappear as these individuals transition into adulthood. Methods: In order to identify areas in need of improvement, this study surveyed 38 parents of post- and peri-transitional young adults aged 18 to 29 . This sample population was drawn from a national advocacy group (National Down Syndrome Congress) and Down syndrome clinics across the nation. Participants completed a written or online survey in which they selected and ranked their goals, and described resources that are beneficial and are barriers to success. At the end of the written survey, participants were invited to take part in a semi-structured phone interview to expound upon their experiences. 38 parents took the written survey and 13 took part in the phone interview. Results: Ultimately, many of the goals parents reported to be most important, including friendships, safety, paid employment, independent living, and access to healthcare, were amongst the least frequently achieved. A number of barriers, including lacking access to transportation, housing, and social options, were found to contribute to this disparity. Study participants also conceptualized new paradigms regarding independence and autonomy. Conclusions: Many of parents most desired goals and dreams for their young adult sons and daughters with Down syndrome are largely going unachieved. Unfortunately, a number of resources are currently insufficient. As a result, practice implications for genetic counselors and other health professionals, educators, and government liaisons include increased assistance with transition planning and increased awareness of appropriate referrals and post-transitional recommendations.

\title{
Exploring Patient Reactions about Genetic Testing for Treatment Response to Bariatric Surgery
}

\author{
L. Balay ${ }^{1}$, S. Aufox ${ }^{2}$, M. Smith ${ }^{2}$, R. Kushner ${ }^{3}$, L. Rasmussen-Torvik ${ }^{4}$ \\ 1. University of Illinois at Chicago \\ 2. The NUgene Project, Center for Genetic Medicine at Northwestern University \\ 3. Northwestern Comprehensive Center on Obesity \\ 4. Northwestern University Feinburg School of Medicine, Departm
}

Bariatric surgery is a common but highly invasive and expensive treatment for obesity. Following surgery, many individuals experience some level of treatment failure. Therefore, methods to ascertain who is the most likely to benefit from the surgery or provide more targeted post-surgical follow-up are highly desirable. Genome-wide association studies and candidate gene studies have provided evidence linking specific genetic variations with outcomes after bariatric surgery. It is unknown what patient perspectives are regarding genetic testing related to bariatric surgery outcomes. This pilot study assessed patient opinions on predictive testing for outcomes after bariatric surgery. Fifteen participants of a genetic biobank, who had undergone bariatric surgery, were interviewed. Twelve themes surfaced around patient understanding of genetic versus non-genetic predictors of weight loss, as well as around patient concerns, interest, and willingness to inform behaviors based on this type of predictive testing. Patients expressed interest in predictive genetic testing for a variety of uses in their own care. Furthermore, many participants held views about predictive testing that were conflicting. While patients discussed genetic predictors in a deterministic manner, they also stated their willingness to use results to change their behavior. Overall, patients did not express concerns around this type of predictive testing (for treatment responses) with few exceptions. Finally, patients suggested that genetic explanations in the clinical setting be provided by medical professionals who have the training to clearly explain genetic concepts and the freedom to provide such explanation with fewer time restrictions. Patient's expressed that these desires were due concerns of their lack of personal genetic knowledge. Overall, these results support the presence of genetic counseling professionals on multidisciplinary teams when providing predictive genetic testing and may eventually aid in the design of trials for genetically tailored care for obesity and other chronic diseases.

\section{Genetic Counseling Clients' Views on Religious and Spiritual Assessment in Genetic Counseling}

\section{A. Bartenbaker ${ }^{1}$, V. DeGifis ${ }^{1}$, A. Trepanier ${ }^{1}$}

1. Wayne State University

Spirituality and/or religion (SR) has been shown to affect coping, support, risk perception, and decision making in healthcare. Purpose: This study aimed to explore whether genetic counseling clients view themselves as spiritual and/or religious, to what extent SR affects their responses to genetic conditions, whether they want their SR needs addressed, and if so under what circumstances. Methods: Approximately 500 members of the Genetic 
Alliance Community Forum Listserv were sent an email inviting them to take part in an online survey. Only those who had had genetic counseling were eligible. The 27-item survey instrument was adapted from previously published work investigating SR in primary care and included multiple choice, Likert-like and open-ended questions. Results: A total of 110 individuals consented to take part; 76 had had genetic counseling and completed at least part of the survey. Most $(86.8 \%, 66 / 76)$ reported they were spiritual or religious to some degree. Of these respondents, a majority indicated that SR affected coping and support from somewhat of an extent to a significant extent $(75.4 \%(46 / 51), 75.0 \%(45 / 60)$, respectively). Conversely, a majority reported that SR had limited to no impact on treatment adherence $(55.0 \%, 33 / 60)$ or risk perception $(53.3 \%, 32 / 60)$. Respondents were split regarding the impact of SR on decision-making. Church attendance was positively correlated with the impact of SR on all five variables ( $p<0.020$ for each). A subset of respondents $(42.6 \%, 26 / 61)$ wanted the genetic counselor to discuss the clients' SR beliefs if they had any; however, this dropped to $18.2 \%(8 / 44)$ if it meant spending less time discussing medical information. Open-ended responses revealed that respondents wanted their SR needs addressed when discussing pregnancy termination or a life-limiting diagnosis or when they initiate the discussion. Conclusions: Many genetic counseling clients may view themselves as spiritual/religious and their SR may affect their responses to genetic disease. Discussing SR may thus be important in promoting adaptation.

\title{
The Psychosocial Implications of Hereditary Diffuse Gastric Cancer
}

\author{
M. Beaston-Casey ${ }^{1}$, D. Patel $^{2}$, E. Lambert ${ }^{3}$, J. Tsipis ${ }^{1}$
}

\section{Brandeis University}

2. MGH - Center for Cancer Risk Analysis

3. Board Chair, No Stomach For Cancer ${ }^{\circledR}$

Identification of an inherited mutation for hereditary diffuse gastric cancer (HDGC) in an individual raises the possibility of a prophylactic total gastrectomy (TG), an unfamiliar, sometimes traumatic experience that reduces cancer risk but also impacts quality of life in significant ways. Few studies have looked at the psychosocial ramifications of learning that one is a HDGC carrier, the decision to have a TG, or the impact the surgery can have on a family. This study aimed to explore families' experiences around genetic testing and prophylactic TG from a variety of perspectives, including but not limited to those having a TG and their caregivers. Through nostomachforcancer.org, we recruited individuals from families in whom a $\mathrm{CDH} 1 \mathrm{mutation}$ had been identified. We used an anonymous, online survey consisting of open-ended questions to eliciting advice for individuals considering genetic testing, considering a TG, and/or those providing care to someone having a TG. We also elicited advice for health professionals guiding HDGC families. Content analysis of 84 responses using SPSS revealed the majority favor genetic testing and TG, focusing on rewards such as life extension. Respondents gave practical advice as well, such as enjoying food pre-surgery and obtaining vitamin supplementation post-surgery. Family members were warned not to minimize their loved one's surgery as simply a procedure to save their life. Advice to caregivers focused on the uniqueness of TC, for example as a "completely alien" experience, and the importance of caregiver self-care. For professionals, nutrition, the emotional recover, and family planning were considered by our respondents as critical issues to address. These results suggest professionals need more knowledge about the physical/emotional aspects of dealing with HDGC, and the unique dietary requirements associated with TG. Additionally, educating family members about physical/ emotional challenges is critical as they provide daily support through this challenging journey.

\section{An Investigation into the Factors that Influence Parental Decision to Disclose Carrier Status to Daughters in Families with Hemophilia}

\author{
K. A. Bisordi ${ }^{1}$, C. Blout ${ }^{2}$, M. Heiman ${ }^{3}$, S. Dixon ${ }^{4}$, J. Scott ${ }^{4}$ \\ 1. University of Maryland, Baltimore Genetic Counseling Program \\ 2. John Hopkins McKusick - Institute of Genetic Medicine \\ 3. Indiana Hemophilia \& Thrombosis Center, Inc. \\ 4. Department of Pediatrics, University of Maryland
}

Communication of genetic information is an essential component of genetic counseling and research. Studies have researched dissemination of medical information following a new genetic diagnosis and of genetic risk information for familial cancer syndromes; however, there is a void in the literature when it comes to research regarding hemophilia, and specifically the disclosure of carrier status. We surveyed parents in families with hemophilia about the factors that influenced their decision regarding when to disclose carrier status to their daughters who are at risk to be carriers of hemophilia. Sixteen parents, recruited through regional National Hemophilia Foundation chapters, participated in an anonymous online survey. This survey aimed to assess parental and daughter-specific characteristics that influence the decision of when to disclose to their daughters three stages of information: what is hemophilia, the inheritance of hemophilia, and daughter's carrier status. It also aimed to determine the role that genetic counselors play in the disclosure process. The results indicated that $68.75 \%(11 / 16)$ of daughters have been told at least one stage of information. Age significantly predicted whether or not they had been told $(p=0.001)$. Parental characteristics such as communication style and comfort levels with understanding and explaining hemophilia inheritance was not found to significantly impact the disclosure process $(p>0.05)$. Many factors are considered important; however, the top three factors were the daughter's exposure to hemophilia through affected relatives, her emotional maturity level, and her age. The majority of parents $62.5 \%$ (10/16) have not seen a genetic counselor, indicating that the role of genetic counselors in the process of parental disclosure can be expanded. Finally, this study identified multiple topics of information that can be provided to parents as patient educational materials to aid in the disclosure process.

\section{SCN5A: A Complex Channelopathy Gene with Counseling Challenges}

D. Clements ${ }^{1}$, P. Aziz ${ }^{1}$, R. Moran ${ }^{1}$, S. Baskar ${ }^{1}$

\section{Cleveland Clinic Foundation}

Changes in the SCN5A gene can lead to a wide range of cardiac concerns, including heart rhythm disorders, dilated cardiomyopathy and sudden infant death. This variability is attributed in part to the ion channel function and the interaction of proteins with the large sodium channel complex. When more 
than one $S C N 5 A$ change is present, the genotype-phenotype relationship is critical for treatment and genetic counseling. We report an 11-year old girl with two syncopal episodes while at rest. She had been evaluated 2 years earlier for concerns of a murmur. At that time she was noted to have Premature Atrial Contractions and sinus arrhythmia but was lost to follow up. At our evaluation, she was diagnosed with sinus node dysfunction, atrial tachycardia with intermittent atrial standstill. Due to her absence of atrial capture, a pacemaker was implanted. Her family history was significant for multiple maternal relatives with syncopal events. Atrial standstill is a rare condition characterized by the absence of electrical and mechanical activity in the atria. It can be secondary (Ebstein's anomaly, Emery-Dreifuss muscular dystrophy, Kugelberg-Welander syndrome, amyloidosis) or familial with variable penetrance when associated with SCN5A gene changes. Genetic testing revealed two alterations in the SCN5A gene: A maternally inherited disease causing frame-shift mutation c.3142 3153del12ins11 which has been seen in a patient with Brugada syndrome and a paternally inherited variant of uncertain significance c.659C $>$ T. This variant has been reported in different clinical phenotypes when present with other SCN5A mutations. Family cardiac screenings showed the mother with asymptomatic atrial flutter and mild right atrial dilatation; the father with normal screening and the asymptomatic 15 year old sister with an incomplete right bundle branch block with the maternally inherited frame-shift mutation. This case illustrates the complexity of sodium channelopathies and highlights the importance of family history and genetic testing when providing genetic counseling.

\title{
Exploring Communication about Type 2 Diabetes and Perceptions of Risk Reduction Methods in Unaffected First Degree Relatives
} of an Affected Individual

\author{
S. Fernandes ${ }^{1}$, L. Koehly ${ }^{2}$, L. Arduser ${ }^{3}$, J. Hopper ${ }^{4}$, M. Myers ${ }^{5}$ \\ 1. University of Cincinnati/Cincinnati Children's Hospital \\ 2. Social and Behavioral Research Branch, National Human Genome Research Institute \\ 3. University of Cincinnati \\ 4. Cincinnati Children's Hospital Medical Center \\ 5. University of Cincinnati Genetic Counseling Graduate Program
}

Despite the known association between family history and type 2 diabetes (T2D) risk, family history is underutilized for identifying and targeting risk reducing interventions. However, family communication about T2D may increase awareness about T2D risk and motivate at-risk individuals to make lifestyle changes. This study investigated T2D communication and perceptions of risk reduction methods among unaffected first-degree relatives of an individual with T2D. Semi-structured qualitative telephone interviews were conducted with 33 unaffected adults recruited from the community. Two researchers used deductive and inductive codes to uncover themes from the interviews. The second researcher randomly coded $40 \%$ of transcripts; all themes were in agreement above $\mathrm{K}=0.7$. Communication themes included topics of discussion, barriers to communication, and ways to improve communication. Diet was the most common topic discussed among families, both as a way to manage T2D and reduce T2D risk. The most commonly mentioned barrier was that conversations about T2D were not relevant to unaffected family members. Education to increase awareness, encourage adoption of risk reducing behaviors, and reduce stigma associated with T2D were suggested as ways to increase T2D communication. When asked about ways to reduce risk, all participants mentioned changing lifestyle. Some suggested identifying relatives to model healthy habits as a way to change lifestyle. Thus, identifying individuals within families to serve as role models and communicate information about T2D with family members may be one strategy. More research is needed to understand how best to improve communication and risk reduction in families with T2D. Genetic counselors should assess a patient's risk of T2D based on family history and advise patients to talk to their family members about T2D to raise awareness of risk.

\author{
Assessing the Impact of BRCA Testing Decisions on Breast Cancer Worry, Decision Regret and Cancer Risk Management \\ J. Frank ${ }^{1}$, W. Uhlmann' ${ }^{1}$, K. Kidwell ${ }^{2}$, S. Merajver ${ }^{1}$, K. Milliron ${ }^{3}$ \\ 1. University of Maryland Medical Center \\ 2. University of Michigan School of Public Health \\ 3. University of Michigan
}

Introduction: Existing literature suggests that cancer worry is a major influence of management choices and few patients regret BRCA testing. The perspectives of patients who decline BRCA testing remain unclear. This study characterized the impact of genetic testing decisions on cancer worry, decision regret, and cancer risk management. Hypothesis: We hypothesized that patients with a BRCA mutation will be most worried about their cancer risk, and regret will be an uncommon outcome of genetic testing decisions. Methods: We analyzed survey data from 222 patients of the University of Michigan Breast and Ovarian Cancer Risk Evaluation Program who completed both the pre- and post (1 year) genetic counseling surveys that assessed cancer worry, decision regret, and cancer management. We grouped patients by their cancer history, genetic testing decision, and test results. Results: Patients who did not get testing had higher decision regret scores after 1 year $(p<0.01)$. Pre-counseling, patients with a personal cancer history had higher cancer worry $(p<0.01)$. Patients who tested positive for a BRCA mutation showed no decrease in their cancer worry over time $(p>0.05)$. Patients who tested negative or declined testing had a decrease in cancer worry $(p<0.01)$. There were no differences among patients' worry after 1 year $(p=0.10)$. There was a high uptake of cancer screening $(N=198,89.1 \%)$, with lower uptakes of chemoprevention $(N=18,8.1 \%)$ and prophylactic surgery $(N=31$, $14.0 \%$ ). Conclusions: Cancer worry and decision regret were low after 1 year. Most patients had a decrease in cancer worry with the exception of those with a history of cancer. Genetic testing decision and test result had no impact on worry or regret. Patients who declined testing had significantly higher decision regret scores. Patients were accepting of increased cancer screening.

Parental Gender Differences: Perception of a Child Diagnosed with a Craniofacial Difference and Effects on Child Adjustment

J. Harris ${ }^{1}$, J. Bodas ${ }^{1}$, J. Rhodes ${ }^{1}$, R. Gannaway ${ }^{1}$

1. Virginia Commonwealth University 
Purpose: To determine if perception and stress is different based on parent gender for individuals with a craniofacial difference. Craniofacial conditions are common birth defects. Maternal stress and perception is linked to child maladjustment in this population. Paternal perspectives have not been reported. Studies show that parents with chronically ill children differ by gender in stress and perception. Method: A retrospective chart review of 37 parents who visited the craniofacial clinic was completed and demographic variables collected. The Strength and Difficulties Questionnaire measured child adjustment, the Impact on Family Scale evaluated the impact on the family, and the Parenting Stress Index evaluated parental stress. A T-Test was performed on responses to determine differences in perception. A two-way factorial ANOVA was performed to identify demographic information that interacts with parent gender on responses. Results: Gender differences were identified in response to two items of prosocial behavior for the child with fathers reporting lower scores than mothers. Fathers also reported of less impact on work and less concern of their child to be treated "specially" than mothers. Demographic variables were analyzed for interactions with parent gender. We observed that when the child was diagnosed with a genetic syndrome, parents regardless of gender reported higher impact on the family $(p=0.038)$ as well as increased stress $(p=0.045)$. Conclusion: Parental gender differences were observed in the way a child is perceived socially, in the impact of a parent's work, and the treatment of the child. This study also demonstrated that other factors such as syndromic status are significant contributors to stress and impact. Many genetic syndromes are associated with craniofacial differences. It is important for genetic counselors to understand the contributions on perception and stress of both parents to provide appropriate counseling and support.

\title{
Communication of Psychiatric Risk in 22q11.2 Deletion Syndrome
}

\author{
S. Hart ${ }^{1}$, K. Schoch ${ }^{2}$, V. Shashi ${ }^{2}$, N. Callanan ${ }^{3}$ \\ 1. Genetic Counseing Program, University of North Carolina at Greensboro \\ 2. Department of Pediatrics, Duke University \\ 3. University Of North Carolina At Greensboro
}

Introduction: Individuals with 22q11.2 deletion syndrome (22q11.2DS) are estimated to have an increased chance of developing schizophrenia or a psychotic disorder. While parents of children affected by 22q11.2DS typically receive counseling about risk for non-psychiatric health concerns, genetic counselors may be reluctant to discuss psychiatric risk. Further education of genetic counselors may be necessary to encourage discussion of psychiatric risk with these families. Purpose: The goal of this project was to develop potential recommendations for genetic counselors to provide psychiatric risk information to families affected by 22q11.2DS. Methods: The recommendations were developed by synthesizing resources in the literature about risk communication. These recommendations were refined following an online focus group meeting with five health care professionals who were recruited for participation from 22q11.2DS clinics across the U.S.A. and had provided care in the past year for at least one individual with 22q11.2DS. The focus group included discussion of key questions related to recommendations for psychiatric risk communication in 22q11.2DS. The transcribed audio recording was analyzed qualitatively and categorized according to the topic being discussed. Results: The focus group data revealed three major themes related to discussion of psychiatric risk: 1) Stepwise approach, 2) Discussing treatment options and minimizing risks, 3) Addressing stigma. Participants endorsed disclosing some psychiatric risk information at diagnosis with more information presented at follow-up sessions. Participants also endorsed providing management recommendations and suggested strategies to reduce feelings of stigma toward mental illness. Conclusion: The final set of recommendations may be used as a foundation for a future clinical protocol to encourage discussion about the risk for mental illness at an earlier point in the diagnostic process for 22q11.2DS and to provide improved information, support and resources to affected families.

Factors Influencing Decisions to Undergo Preimplantation Genetic Diagnosis for Fanconi Anemia and Long-Term Interpersonal Outcomes of those Decisions: A Qualitative Investigation of Parents' Experiences

\author{
K. Haude ${ }^{1}$, B. LeRoy ${ }^{2}$, P. McCarthy-Veach ${ }^{3}$, H. Zierhut ${ }^{2}$ \\ 1. University of Minnesota, Twin Cities \\ 2. Department of Genetics, Cell Biology and Development, University of Minnesota \\ 3. University of Minnesota
}

Fanconi anemia (FA) is characterized by congenital malformations, progressive bone marrow failure, and predisposition to malignancy. There are risks associated with hematopoietic stem cell transplantations, and best results are attained with sibling donors who are human leukocyte antigen (HLA) identical matches. Preimplantation genetic diagnosis (PGD) offers parents the opportunity to have a healthy child who is an HLA match. While some research has investigated parents' experiences throughout the PGD process, no published studies specifically address factors influencing their decisionmaking process and long-term interpersonal outcomes. This study investigated perspectives of parents who had a child born using PGD for FA and/or HLA typing. There were three aims: (1) examine parents' expectations and the influence of media, bioethics, and religion on their decision-making process; (2) examine parents' social support and emotional experiences throughout their PGD process; and (3) characterize long-term effects of PGD on relationship dynamics (partner, family, friends), others' attitudes, and parental regret. Nine parents participated in semi-structured interviews. Thematic analysis revealed their decision to use PGD was influenced by media, bioethics, and religion, particularly affecting parents' initial confidence levels. Moreover, the PGD process is emotionally complex, with parents desiring varying amounts and types of support from different sources at different times. For instance, parents desired support while undergoing in vitro fertilization and PGD and wanted help caring for their child affected with FA. Long-term effects of PGD generally have been neutral or positive for family dynamics, and negative or positive for friendships. Parents reported others' attitudes towards them are respectful or no different than before PGD. Parental regret regarding PGD was negligible. Results of this study will promote optimization of long-term care for FA families. Additional findings, and genetic counseling practice and research implications are presented.

\section{Confirmed versus Suspected: The Social Significance of a Genetic or Non-Genetic Diagnosis of Mitochondrial Disease}

E. Krieg', K. Krepkovich ${ }^{2}$, L. Calderwood ${ }^{3}$, M. Campion ${ }^{1}$ 
1. Boston University School of Medicine

2. Boston University

3. Boston Children's Hospital

Objective: To investigate how the attitudes and beliefs regarding the importance of a genetic versus non-genetic diagnosis impact an individual's support needs and interactions within the mitochondrial disease community. This study explored the impact of diagnosis type on various aspects of the patient experience by identifying and comparing patient sub-groups within the mitochondrial disease population. Methods: The study population $(n=201)$ was surveyed via an online survey. Respondents were categorized into two groups, those with a genetic diagnosis of mitochondrial disease and those with a non-genetic diagnosis. Survey responses were compared between the two groups using methods of quantitative and qualitative analysis. Results: The two patient groups have different support needs but are both fairly significantly affected by mitochondrial disease and experience similar problems relating to medical providers, costs of care, lack of appropriate support resources, and concerns regarding available treatment. The non-genetic group faces additional challenges related to not having a confirmed genetic diagnosis. Conclusion: There are significant problems with the perceived quality of care for both groups but overall, individuals with a non-genetic diagnosis experience more problems and unmet needs in areas related to both medical and social support due to their lack of a definitive diagnosis. Support resources for the non-genetic diagnosis group must be designed to address the unique challenges associated with not having a confirmed genetic diagnosis. Practice Implications: It is important to understand the efficacy of existing patient support resources for different sub-groups within the mitochondrial disease patient population so that appropriate services and resources can be developed or improved to address specific needs of that sub-group and provide effective patient care and support.

\title{
Who Should I Bring? A Qualitative Investigation of Genetic Counselors' Perspectives on the Role of the Support Person in Cancer Genetic Counseling Sessions
}

\author{
R. Swartwood ${ }^{1}$, P. McCarthy-Veach ${ }^{1}$, Y. Sun ${ }^{1}$, M. Truelson ${ }^{1}$, M. Ahrens ${ }^{1}$, B. LeRoy ${ }^{2}$ \\ 1. University of Minnesota \\ 2. Deptartment of Genetics, Cell Biology and Development, University of Minnesota
}

Perceived social support positively predicts healthcare outcomes. Yet, only one study, of Huntington's Disease, specifically examines the support person's role in genetic counseling sessions. The present study investigated the support person's role in cancer genetic counseling from the perspective of practicing genetic counselors. There were three major research questions: (1) How do genetic counselors involve the support person in patients' genetic counseling sessions? (2) What variables do genetic counselors believe contribute to successful and unsuccessful support person involvement? and (3) How can genetic counselors help patients construct the best psychosocial support within sessions and after the genetic counseling relationship ends? Fourteen cancer genetic counselors engaged in semi-structured, phone interviews about their: approach to talking to patients about bringing a support person to session(s), impressions of patients' decision-making process vis a vis choosing a support person, successful and unsuccessful support person involvement, and perceived obligations to the support person. Using grounded theory analysis data were organized into themes supporting a core category (general theory). The derived core category corresponds to Lakey and Orehek's Relational Regulation Theory tenets: social support buffers against negative patient reactions, and perceived support comprises the mechanism through which buffering occurs. Specific to this study, findings indicate support persons are involved most successfully when they are: 1) viewed as supportive, 2) are aligned with the patient's agenda, and 3) emotionbased, information-based, focused on decision-making, or some combination of these. Additional findings regarding successful and unsuccessful support person qualities, patients' decision-making about selecting a support person, and genetic counselor strategies to facilitate positive support person experiences during and after sessions are presented.

\section{An Evaluation of Genetic Counseling Effectivity as Perceived by Parents with Surviving Children of Trisomy 13, Trisomy 18, and Mosaic Trisomy 16}

\author{
T. J. Lewis ${ }^{1}$, M. Dudek², A. Fair ${ }^{3}$, F. Ampy ${ }^{4}$, B. Harrison', \\ 1. Department of Genetics and Human Genetics, Howard University \\ 2. Department of Obstetrics and Gynecology, Vanderbilt University \\ 3. Department of Research Support Services, Vanderbilt University \\ 4. Department of Biology, Howard University
}

Background: Survival from a lethal genetic condition has been referred to as a "medical mystery". Survival of lethal genetic conditions are considered rare occurrences, therefore, there is a paucity of descriptive survival data within the scientific literature. Purpose: This study examined whether parents perceived they were adequately informed at genetic counseling for the circumstances surrounding a child who survived with trisomy 13, trisomy 18, or mosaic trisomy 16. Methods: Study participants completed a one-time, anonymous, online survey. Results: Data of twenty mothers from support groups revealed mothers with children of these diagnoses, had overall lower levels of perceived parental uncertainty. The qualitative data analysis illustrated the mothers' likes, dislikes, and recommendations for future genetic counseling sessions. Conclusions: Quantitative data results revealed no significance in the variances of perceived personal control and perceived uncertainty when evaluated on the factors of education, country of origin, and whether the affected pregnancy was the first pregnancy. The study identified seven themes in the qualitative data. These themes included information given within the counseling session, research presented, the personality and approach of the medical professional, the outcomes of the diagnosis, testing options, referral, and personalized care. Future directions: More research is needed to evaluate the necessity of adapting new techniques to counseling in rare cases of survival based on input from fathers and medical professionals. The qualitative data from this study can be useful in informing the literature for creating an adaptive genetic counseling tool to be used for preparing parents of a child who has survived trisomy 13,18 or mosaic trisomy 16 . 


\title{
The Role of Uncertainty in Coping Efficacy: The Experience of Parents of Children with Undiagnosed Medical Conditions
}

E. Macnamara ${ }^{1}$, P. K. J. Han², S. Lipinski ${ }^{3}$, B. Biesecker ${ }^{4}$

1. National Human Genome Research Institute, National Institutes of Health

2. Maine Medical Center Research Institute

3. Department of Pediatrics, University of Virginia

4. Genetic Counseling Training Program, Johns Hopkins University/National Human Genome Research Institute

The purpose of this study was to understand what uncertainties are perceived by parents of children with undiagnosed medical conditions, what factors contribute to perceptions of uncertainty and how this uncertainty affects coping efficacy. A cross-sectional, mixed methods study was conducted to explore the relationships among perceptions of uncertainty, coping efficacy, coping, and personality traits: tolerance of uncertainty, resilience, and optimism. The study design was informed by Lazarus and Folkman's Transactional Model of Stress and Coping. Measures included a newly developed Parental Uncertainty of Children's Health Scale that examined parents' perceptions of various uncertainties and the importance of resolving these uncertainties. Parents of children with undiagnosed medical conditions were recruited through online support and advocacy groups. Among the 94 respondents, the majority were biological mothers $(94 \%)$, Caucasian $(94.7 \%)$ and married $(76.6 \%)$. A slight majority of the children were female $(57.6 \%)$. They were, on average, 8.0 years of age. Parents perceived greater uncertainty than certainty about areas of their child's undiagnosed condition that were important to them. Multivariate analysis revealed that higher levels of optimism were associated with less perceived uncertainty $(p<0.01)$, and that perceptions of uncertainty, optimism and resilience predicted coping efficacy $(p<0.05)$. Analysis revealed that perceptions of uncertainty are associated with appraisals of coping efficacy such that higher perceptions of uncertainty resulted in lower coping efficacy. Our findings suggest that parents of children with undiagnosed medical conditions perceive significant uncertainty about social support and medical management, which they view as important to resolve. Personality traits are related to the type of coping strategies parents employ. Finally, this study contributes to the broader understanding of perceptions of uncertainty and the potential impact of these perceptions for parents of children with undiagnosed medical conditions.

\section{Duchenne Muscular Dystrophy: A Survey of Families' Perspectives on Carrier Testing and Communication within the Family}

\section{B. Mellicker ${ }^{1}$, S. Hassed ${ }^{1}$, J. L. Chaloner ${ }^{2}$, C. Guy ${ }^{1}$}

\section{University of Oklahoma}

\section{Perinatal Center of Oklahoma}

Previously published literature describes family perspectives on carrier testing for X-linked diseases in minors and communication within the family but perspectives related to an X-linked lethal disorder have yet to be described. This study aimed to identify the perspectives of individuals with a family history of Duchenne muscular dystrophy on the preferred timing of carrier testing and experiences discussing Duchenne muscular dystrophy within the family. Twenty-five women over the age of 18 who have a son with Duchenne muscular dystrophy participated in an online survey. The survey consisted of multiple choice and open ended, short answer questions. Results were analyzed using thematic analysis. Participants were mothers of boys with Duchenne muscular dystrophy. Themes regarding reasons to perform carrier testing prior to eighteen years of age included: teenage pregnancy rates, increased knowledge about reproductive options, and concerns regarding daughter's ability to emotionally cope with the information. Themes regarding communication styles within the family included: open and honest communication to their children throughout their lives. Themes regarding communication with a genetic counselor included: positive feelings regarding information obtained and negative feelings regarding going against parent wishes due to age of daughter. Mothers indicated that they desire autonomy in deciding when their daughters should have carrier testing and discussing carrier testing with their daughters. Genetic counselors should incorporate this information into their genetic counseling practice in order to increase the effectiveness of the genetic counseling session.

\section{The Impact of Culture and Ethnicity on the Counseling Process: Perspectives of Genetic Counselors from Minority Ethnic Groups}

\author{
B. Morris ${ }^{1}$, C. Hill-Chapman ${ }^{2}$, B. Harrison ${ }^{3}$, T. Hardy ${ }^{2}$ \\ 1. Genetic Counseling Program, University of South Carolina \\ 2. Francis Marion University \\ 3. Howard University
}

Introduction: Cultural competency emphasizes the importance of patient culture and provider culture in a counseling session. Competent counselors should be aware of the influence of their personal cultural values on interactions with patients and peers. Few studies have examined the cultural influences of counselors from minority ethnic groups. Purpose: This study sought to evaluate the influence of counselors' cultural values and ethnic identity on their approach to genetic counseling. Methods: 162 genetic counselors, 58 self-identified as individuals from ethnic minorities, completed an online survey that contained the validated Multigroup Ethnic Identity Measure scale, a segment of the Benet-Martinez Acculturation scale, demographic data, and questions regarding patient preference and cross-cultural counseling. 21 counselors participated in semi-structured telephone interviews to discuss cultural values, cultural competency, counseling techniques, and experiences surrounding cross-cultural counseling. Results: Most counselors $(77.6 \%)$ do not have a preference for counseling patients from similar backgrounds. Patient preference and cross-cultural changes in counseling were not significantly associated with ethnic identity or acculturation; younger, less experienced counselors were more likely to alter their counseling crossculturally. Counselors described family dynamics and education as cultural values that directly influence their approach to genetic counseling, and generally felt that the needs of the patient were more influential than the cultural similarity between them. Conclusion: These findings support the practice of comprehensive training in patient education and interpreting family dynamics. Awareness of common counselor characteristics such as empathy or education may allow for more effective recruitment of individuals who may be naturally drawn to the field. Cultural competency efforts have had a positive impact; increased efforts in clinical, didactic, and recruitment realms would be beneficial. 
How Does Family Communication about Cancer Work? Exploration of a Mediational Model

J. Quillin ${ }^{1}$, M. Gyure ${ }^{1}$, D. McClish ${ }^{1}$, R. Corona ${ }^{1}$, A. Krist ${ }^{1}$, J. Borzelleca ${ }^{1}$, A. Maibauer ${ }^{1}$, D. Bowen ${ }^{2}$, J. Bodurtha ${ }^{3}$

1. Virginia Commonwealth University

2. Boston University

3. Johns Hopkins University

Accurate family health histories depend on good client-family communication. Studies have identified several family communication predictors including family (e.g., closeness), client (e.g., gender), and provider (e.g., recommendation) characteristics/influences. More evidence is needed, however, to understand how family communication works following an intervention such as genetic counseling. We sought to identify mediators that explain family communication about cancer after a clinic-based intervention that included several characteristics of traditional genetic counseling. The KinFact study randomized 490 Women's Health Clinic patients to receive cancer risk assessment and family communication skills training. Participants completed one-, six-, and 14-month follow-up interviews. Outcomes included measures of family history collection ("Gather") and sharing information about familial risk ("Share"). A composite measure assessed frequency of communication ("Frequency"). Tested mediators were self-efficacy, perceived breast/colon cancer risk, knowledge, and genetic testing attitudes. Our previous analyses showed family communication occurred by the one-month follow-up and did not increase at later time points; therefore, we analyzed mediator and outcome effects at the one-month follow-up. Bootstrap methods were used for inferences about the indirect effects. Communication self-efficacy was a statistically significant mediator (OR=1.12 for "Gather", 1.17 for "Give", and 1.03 for "Frequency"). Breast cancer knowledge appeared to mediate "Give" (OR=1.09). No other significant mediators were identified. In general, results of this study suggest the mechanism for action of the KinFact intervention remains unknown, as self-efficacy and breast cancer knowledge, although statistically significant mediators, showed small effects, so might not be useful predictors of communication. Since mediators and outcomes were assessed at the same time, we cannot be sure of the direction of causality. Future work might benefit from formative research of communication processes.

\title{
Support Desired by Women following Termination of Pregnancy for a Fetal Anomaly
}

\author{
A. Ramdaney ${ }^{1}$, S. Hashmi ${ }^{1}$, M. Monga ${ }^{2}$, R. Carter $^{1}$, J. Czerwinski ${ }^{3}$ \\ 1. University of Texas Houston \\ 2. Baylor College of Medicine \\ 3. The University of Texas Health Science Center at Houston
}

With the rapidly evolving field of prenatal testing, there is a growing need to provide support for women pursuing a termination of pregnancy following the discovery of a fetal anomaly. Previous studies have documented that women in this situation often feel unsupported after the procedure, but the type of resources desired by this population remains undetermined. A longitudinal study was performed in 51 women terminating for a fetal anomaly at a private clinic at the time of the procedure, at 6 weeks, and at 3 months following the event. Surveys were kept anonymous and investigated the awareness and utilization of support resources as well as influential and preventative factors. Though largely knowledgeable of the existing resources, only $50 \%$ admitted contemplating their individualized need for support. Anonymity, ease of access, and the desire to meet others in a similar situation online were commonly selected as the most influential factors in the decision to participate in support systems. Regardless, most women expected to rely on the support from family and friends. Only four respondents expressed that a lack of support from family and friends would influence them to pursue other support resources. Additionally, $50 \%$ expressed the desire to commemorate the pregnancy, though none wanted direct contact with their healthcare provider(s). The desire to move on from the pregnancy was the most cited factor that prevented consideration of support resources in the follow-up period and seemed more important to this population than other influential factors. However, many women indicated not coping as expected and were unprepared for the psychological consequences following the procedure. Our findings indicate that women in these situations may not realize what their long-term support needs will be. Additional support resources that promote a flexible timeframe for uptake need to be developed to meet the unique desires of this population.

\section{Parent Reflections on the Diagnostic Odyssey}

\author{
A. Richardson ${ }^{1}$, J. Fanos ${ }^{2}$, A. Cherry ${ }^{1}$, A. Kwan ${ }^{1}$ \\ 1. Stanford University School of Medicine \\ 2. Geisel School of Medicine at Dartmouth
}

Purpose: There is a gap in the literature on parents' experiences of their child's diagnostic odyssey involving multiple types of genetic testing. Chromosome microarray analysis is a common genetic test when diagnosis remains elusive. This mixed-methods study asked if diagnosis through chromosome microarray analysis brought closure to parents after a diagnostic odyssey, and explored the impact of testing and diagnosis. Results include recommendations for genetic counseling interventions. Methods: 19 parents of 16 children who had chromosome microarray analysis at Stanford University between 2007 and 2012 participated in semi-structured interviews that included use of the Psychological Adaptation Scale. An iterative coding process was used to identify major themes. Results: Participants' children ranged in age from newborn to 24 years at the time of testing. Diagnostic odysseys lasted an average of 5.1 years. Diagnosis from testing included: 10 copy number variants, 2 structural rearrangements, 2 variants of uncertain significance, and 2 normal results. Only 3 parents reported feeling complete closure post-testing, all of whom had children tested at birth. Scores from the Psychological Adaptation Scale did not correlate with closure. Emerging themes on the impact of diagnosis include: unconditional love, complicated relief, hope and devastation, remaining uncertainty, and opening another door. Parents took on unique roles, including becoming an 'expert', and story-telling to make meaning. Despite frustration at lack of medical utility of diagnosis and persistent uncertainty, all parents recommended testing. Discussion: Conflicting emotions and pervasive uncertainty made it difficult for parents to feel closure after diagnosis, amplified by the length of the 
diagnostic odyssey. Opportunities exist for genetic counselors to improve parent coping and adaptation through anticipatory guidance on the impact of testing, assisting with meaning-making, clarifying results over time, and providing connections to other affected families and specialized support groups.

Teens with Glycogen Storage Disease Types I and III: Planning to Take Responsibility

\author{
H. Rocha ${ }^{1}$, J. Sullivan ${ }^{2}$, S. Austin ${ }^{2}$, N. Callanan ${ }^{3}$ \\ 1. Geisinger Medical Center \\ 2. Duke University Medical Center \\ 3. University Of North Carolina At Greensboro
}

Background: Recently, adolescent transitions in the context of healthcare have begun to gain attention among pediatric providers. General guidelines for developing transition plans were established by the American Academies of Pediatrics in 2002, but specific educational topics covered in each plan will be unique to each patient's diagnosis. Purpose: The goal of this study was to identify issues specific to the glycogen storage disease (GSD) population to create a targeted medical transition program. Methods: Focus group participants were recruited through the Association for Glycogen Storage Diseases listserv. The thirteen participants included professionals, adults with GSD, and parents. The focus group was recorded and transcribed. To identify common themes, the primary investigator and one advisor analyzed the transcription until agreement was reached. Results: Four major themes were identified: monitoring systems, technology and tools, shifting responsibility, and general issues of adolescence. The themes discussed most frequently guided the development of program sections and provided a basis for addressing specific concerns during transition. Selected quotes were used throughout the program to highlight individual experiences. Discussion: To encourage successful self-management for the future, it is the responsibility of the pediatric healthcare team to begin teaching skills that promote independence and responsibility for care. The goal of this program is to provide clinicians with all the necessary tools to teach those skills. Four program sections were designed to address this goal: Introduction to the Teen Transition Plan; Understanding GSD, Your Body, and Alcohol; Body Image and Exercise; and Peer Support and Mentoring. Conclusion: This unique transition program addresses the needs of 11 to 14 year olds with GSD and their families. The study assessed the needs and ideas of a specific population and used relevant information to develop a tool that can be easily implemented into clinical care by any healthcare provider.

\title{
"Who is the Deciding Factor?" Analysis of Parental Perspectives regarding the Discontinuation of Elaprase in Children with Mucopolysaccaridosis Type II
}

\author{
E. Schindewolf ${ }^{1}$, L. Conway ${ }^{1}$, S. Chadwick ${ }^{2}$ \\ 1. Arcadia University \\ 2. Children's Hospital of Philadelphia
}

Mucopolysaccaridosis type II (MPS II) is a rare, progressive, multisystemic lysosomal storage disease that affects mainly boys and can cause progressive neurodegeneration in severe cases. Enzyme replacement therapy, marketed as Elaprase, is clinically available to treat this disorder, but has no impact on neurocognitive symptoms. Because of this, decisions of when to withdraw Elaprase in relation to neurocognitive decline are often difficult. Recent recommendations suggest discontinuation of Elaprase for many reasons including regression to a vegetative state or lack of improvement in quality of life. These guidelines were based on input from clinicians but did not include parental perspectives. To address this gap, 18 parents of children with severe MPS II whose children were receiving Elaprase treatment for at least 6 months were contacted via the MPS Society and through snowball recruitment for participation. Participants completed a semi-structured phone interview which was transcribed and analyzed using grounded theory methodology. Results from this studied indicated that all parents perceived improvements from Elaprase for their child, with the most significant improvements being quality of life and range of motion. In addition, all parents had at least thought about discontinuation of treatment for their child, though many described being unable to make the decision on their own. Many parents believed the guidelines for discontinuation were too strict and that focus should be put on individualized care plans as well as measures like quality of life and disease progression. Parents specifically objected to the recommendation that treatments be discontinued once a tracheotomy has been performed. Parents wanted more opportunities to monitor progression of disease with treating physicians and a greater voice in conversations relating to discontinuing treatment. This study highlights the importance of involving families in the creation of healthcare guidelines and the need for collaboration between health care providers and families.

\author{
Adaptation to Living with a BRCA1 and BRCA2 Mutation in Carriers and Their Partners \\ R. Shapira ${ }^{1}$, L. Erby $^{2}$, L. Hoskins ${ }^{3}$, W. Klein ${ }^{3}$, G. Hooker ${ }^{4}$ \\ 1. St. Joseph Hospital \\ 2. Johns Hopkins Bloomberg School of Public Health \\ 3. National Cancer Institute \\ 4. $N \operatorname{ext} G x D x$
}

Women who carry BRCA1/2 mutations have a significantly elevated risk for breast and ovarian cancer. Following a positive test result, many decisions must be made, among them decisions about surveillance and risk-reducing surgery. Both screening and surgical options can cause distress and anxiety, for the carriers themselves and their intimate partners, and may impact overall adaptation to living at risk. The purpose of this study was to examine psychological adaptation in individuals living with genetic risk for cancer, including dyadic-level analysis of adaptation and adjustment in BRCA1/2 carriers and their partners. The conceptual model is largely based on Lazarus and Folkman's Transactional Model of Stress and Coping, and Bodenmann's conceptualization of the process in couples. Female BRCA1/2 carriers and their partners were invited to complete surveys designed to quantitatively explore the relationships between the appraisals and timing of risk-related stressors, dyadic coping, and the outcomes of adaptation and dyadic adjustment. 103 carriers and 43 partners completed the survey. Women who had undergone prophylactic bilateral mastectomy had significantly 
higher levels of adaptation than those who had not (3.50 vs. $2.53, p=0.000)$. Further, their partners had significantly higher adaptation as well (3.30 vs. $2.37, p=0.001)$. Among women who had not had prophylactic mastectomy, those with higher perceived risk scores were less adapted $(p=0.025)$. In general, the participants' levels of dyadic adjustment and dyadic coping indicated good overall relationship quality. This is the first study to describe adaptation in either $B R C A 1 / 2$ carriers or their partners and aids in the understanding of the experience of living with cancer risk, and the potential relationship between risk management decisions and adaptation. The relatedness of carrier surgical status to partner adaptation points to the importance of including intimate partners in the genetic counseling and risk management decision-making processes.

\title{
Perceptions of Latinas on the Traditional Prenatal Genetic Counseling Model
}

\author{
S. Thompson' ${ }^{1}$, S. J. Noblin ${ }^{2}$, J. Lemons², S. Peterson ${ }^{3}$, C. Carreno ${ }^{2}$ \\ 1. The Graduate School of Biomedical Sciences at Houston, University of Texas \\ 2. University of Texas Health Science Center at Houston \\ 3. MD Anderson Cancer Center
}

The traditional genetic counseling model reflects an individualized counseling session that includes the presentation of information about genes, chromosomes, personalized risk assessment, and genetic testing and screening options. Counselors are challenged to balance providing educational information with discussion of implications of this information in an allotted amount of time. The aim of this study was to explore the perceptions of pregnant Latinas on the benefits and limitations of the traditional prenatal genetic counseling model and to determine the specific preferences for receiving prenatal genetic counseling. Data were collected through focus groups and one-on-one, semi-structured interviews of twenty-five Spanish speaking Latinas who received genetic counseling during their current pregnancy. Their responses were evaluated using thematic analysis to identify major themes in participant responses by utilizing a grounded theory approach. Several themes were identified including an overall satisfaction with their prenatal genetic counseling appointment, desire for a healthy baby, peace of mind following their appointment, no desire for invasive testing, and faith in God. Several participants also stated a preference for group genetic counseling over the traditional individual genetic counseling model. Our data indicate that Latinas value the information presented at prenatal genetic counseling appointments despite disinterest in pursuing genetic testing or screening and suggest that group prenatal genetic counseling may be an effective alternative to the traditional genetic counseling model in the Latina population.

\section{Exploring Fathers' Roles and Experiences with Dissemination of Sexual Health Information to Their Children with Down Syndrome}

\author{
L. Torrey ${ }^{1}$, S. Cullen ${ }^{2}$, A. Cirino ${ }^{3}$, M. Campion ${ }^{4}$ \\ 1. Boston University \\ 2. Massachusetts Down syndrome Congress \\ 3. Brigham and Women's Hospital \\ 4. Boston University School of Medicine
}

Sexual exploration is an essential part of human development during adolescence and young adulthood. While typically developing children may inherently understand the social nuances of relationship building, children with intellectual disability (ID) such as Down syndrome often require more direct instruction. Prior studies on parental views of sexual education of children with disabilities have excluded fathers citing perhaps outdated perspectives of parenting roles. This exploratory study aimed to identify the modes some fathers use to explain sexual health information to their children with Down syndrome. Goals were to understand when and how this dialogue is initiated, resources fathers utilize to inform their conversations, and the concerns, challenges and advice fathers wish to share with other fathers. Key informants affiliated with the Massachusetts Down syndrome Congress (MDSC) who also had children with Down syndrome helped conduct purposive recruitment. Semi-structured interviews were conducted with two fathers of 16 year olds with Down syndrome, one a boy and the other a girl. Participants were asked to reflect on experiences discussing sexual health with their children. Interviews were audio-recorded, transcribed verbatim, coded, and analyzed using modified grounded theory techniques. Participants reported using specific strategies to discuss sexual health content with their children including repetition, contextualization, and role-play. To inform their conversations fathers utilized workshops and seminars, their co-parents, and personal reflections of adolescence. Salient themes emerged on the conflict of their children's access to sexual information and intimacy with safety fears. This pilot study is the first to directly address fathers' experiences discussing sexual health information with their children. This research highlights the complex relationships some fathers have with the sexual health information dissemination process, and broadens the dialogue on parent-child gender dyads to include families of children with disabilities.

\section{Predictive Testing for Huntington's Disease: An Exploration of the Partner's Role in Decision-Making}

\section{S. Towner ${ }^{1}$, D. Rintell ${ }^{1}$, S. Berg ${ }^{2}$, K. Sullivan-Freimuth ${ }^{3}$, J. Freimuth ${ }^{3}$, J. Tsipis ${ }^{1}$}

1. Brandeis University

2. Dartmouth-Hitchcock Medical Center

3. Patient Advocate

Huntington's disease (HD) is a progressive neurodegenerative disease that causes motor and cognitive impairment. Predictive testing is available for atrisk individuals who desire to learn whether they inherited the HD gene mutation. Many studies identify factors that influence the decision to undergo predictive testing among those at-risk. Few studies, however, focus on the spouses of these individuals to explore their views regarding predictive testing. The purpose of this study was to elicit spouse's attitudes toward predictive testing, identify factors contributing to their opinions, and better understand their needs throughout the predictive testing process. We recruited individuals through the Huntington's Disease Society of America, Boston Medical Center, and Albany Medical Center. All participants were in a relationship with someone who had undergone presymptomatic predictive testing within the last 10 years. We performed qualitative and semi-structured phone interviews with six such spouses, three women and three men. Several important 
findings emerged from the interviews. First, we found that spouses favored earlier predictive testing than their at-risk partners, yet remained supportive of the decision to delay testing. Second, we identified a lack of understanding regarding the purpose and benefits of pre-test genetic counseling sessions. Two couples opted out of pre-test counseling through an HD center because 'they already knew what HD was,' but received inadequate information and support. Finally, we provide further evidence that spouses feel their individual needs are overlooked following their partner's positive test result. Continued education in the HD community regarding the benefits of pre-test genetic counseling through support groups, online resources, and physician's offices may limit the number of at-risk individual who opt out due to misconceptions. Furthermore, healthcare professionals should remain cognizant of the possibility of distress in spouses and work to increase the availability of spousal specific resources and referrals.

\title{
Education/ELSI
}

\section{Genetic Counselors' Knowledge and Perspectives of Cord Blood Banking and Stem Cell Therapies}

\section{S. Brummitt ${ }^{1}$, H. Harris ${ }^{2}$, B. Kirkpatrick ${ }^{3}$, J. M. Sakr ${ }^{4}$, Youngblom ${ }^{5}$}

\author{
1. CSU Stanislaus \\ 2. Cord Blood Registry \\ 3. Geisinger Health System \\ 4. Integrated Genetics \\ 5. California State University
}

Umbilical cord blood presents a unique opportunity to gather and preserve hematopoietic stem cells for possible treatment of many diseases, including genetic conditions. Ongoing clinical trials are revealing new uses for these stem cells everyday. Genetic counselors play a significant role in prenatal, pediatric and adult care for many patients and families who may benefit from learning about cord blood banking and therapies, however this group has never before been assessed on its knowledge of or opinions on these topics. This study reports on genetic counselors' knowledge and opinions of cord blood banking and stem cell therapies, as well as how often and why they are talking to their patients about it. A survey was administered to members of the NSGC via an e-blast survey which assessed respondents' factual knowledge on cord blood banking as well as their feelings about their own knowledge and need for education on the topic. Results revealed that as a group, genetic counselors have limited knowledge of cord blood banking and stem cell therapies. Most respondents were aware of their lack of knowledge, rating their own understanding of different aspects of cord blood banking as low in spite of their agreement that cord blood banking and stem cell therapies are topics with which genetic counselors should be familiar. The majority of study participants $(67 \%)$ also indicated that they would find an educational booklet useful to their practice. These results highlight an area of need in genetic counselors' education, and the authors provide ideas for focusing future education efforts of genetic counselors on the value of stem cell therapies and cord blood banking.

\section{An Analysis of Online Education Methods for an at-Home Genetic Carrier Screening Service}

\author{
J. Denton ${ }^{1}$, R. Donnell ${ }^{2}$ \\ 1. JScreen, Emory University \\ 2. Emory University
}

JScreen is a nonprofit, online education and carrier screening program for Jewish genetic diseases. Patients request a kit online and receive a saliva-based kit in the mail to screen from home. Pre-test education is essential for understanding carrier screening and result implications. Patients have access to educational materials on JScreen's website; in addition, genetic counselors are available for support. Before receiving a kit, patients watch a 4 min video explaining concepts involved in carrier screening, including: recessive inheritance, test limitations, and Tay Sachs enzyme testing. Patients are emailed a 5 question quiz to ensure they understood the information from the video. If patients require genetic counseling, appointments are done by phone or secure video conference. The following is an analysis of JScreen's educational efforts as of 4/25/14. Fifty-six percent of patients received genetic counseling, including all positive patients and those with negative results who either did not answer or scored $<80 \%$ on the quiz. Video conferencing accounted for $35 \%$ of appointments, and $65 \%$ were consulted via phone. Carrier couples or others requiring additional counseling received referrals to local providers. The majority of patients $(63 \%)$ reported watching the video during the "request a kit" process, and $33 \%$ watched right before the quiz. Of 207 quiz respondents, $76 \%$ scored $5 / 5$ and $21 \%$ scored $4 / 5$, meaning only $4 \%$ of patients scored below $80 \%$. The most common question missed was "what is the chance of a recessive carrier developing the disease." Of those who answered incorrectly, $9.7 \%$ believed the chance was $50 \%$, and $4.8 \%$ answered it was a $10 \%$ chance. Nearly all (98 \%) participants knew that parents who are carriers for the same condition have a 1 in 4 chance to have an affected child. JScreen patients clearly demonstrate retention and comprehension of key concepts involved in carrier screening. JScreen's remote educational techniques significantly decrease the time and cost burden of in-person pre and post-test counseling for carrier screening.

\section{The Impact of Increased Education on Career Interest in the Genetic Counseling Field among High School Students}

\author{
J. Dix ${ }^{1}$, K. East $^{2}$, K. Lee ${ }^{3}$, R. Stewart ${ }^{4}$ \\ 1. The University of North Carolina at Greensboro \\ 2. HudsonAlpha Institute for Biotechnology \\ 3. University of North Carolina at Chapel Hill \\ 4. Genetic Counseling Program, University of North Carolina at Greensboro
}


Introduction: Genetic counseling is a rapidly growing profession. Previous studies identified high school as an ideal time to present genetic counseling as a career option to increase the number of potential applicants, especially among ethnic minorities. To date there is insufficient literature regarding the effect of increased education about genetic counseling upon level of interest in the profession. Purpose The goal of this study was to evaluate whether increased education about the genetic counseling field impacts career interest among high school students. We hypothesized that increased education about genetic counseling would increase students' knowledge about genetic counseling and career interest. Methods: An educational presentation and class activity were designed to provide high school students in the Middle College at The University of North Carolina at Greensboro with information about the genetic counseling profession. Pre- and post-lecture surveys were administered to assess knowledge of and interest in genetic counseling. Paired samples t-tests were used to analyze the statistical significance of related questions on the pre- and post-surveys. Responses to open-ended questions were analyzed to identify common themes. Results: Eighty students participated in the study. Ninety-five percent reported their knowledge of genetic counseling increased and a statistically significant increase in students' interest in genetic counseling as a career was noted $(p<.001)$. Caucasian students felt more familiar with genetic counseling and pedigree construction after the educational intervention as compared with African Americans. Students interested in pursuing a genetic counseling career reported their ability to help and comfort others as the main reason for their interest. Conclusion: Our results suggest that the educational intervention did increase the students' knowledge about genetic counseling and career interest. Future research is needed to enhance the generalizability of our findings and determine if such educational interventions may be helpful to increase ethnic diversity in the profession.

Topics of Discussion in Families with Youth with Special Health Care Needs during Health Care Transition

\author{
C. Grabarits ${ }^{1}$, D. Sarandria ${ }^{2}$, S. Delany Dixon ${ }^{1}$, C. Applegate ${ }^{3}$, K. Morphy ${ }^{4}$ \\ 1. University of Maryland School of Medicine \\ 2. Department of Pediatrics, University of South Florida Health \\ 3. Department of Pediatrics, Johns Hopkins \\ 4. Allegheny General Hospital Cancer Center
}

Healthcare transition is the purposeful movement of adolescents with chronic medical conditions from child-centered to adult-oriented health care systems in hopes of providing comprehensive health care in an uninterrupted manner. Communication is a key aspect of transition; specifically, provideradolescent communication is connected to increased transition counseling and improved health outcomes. However, only $42 \%$ of youth ages $12-17$ had discussed transition with their pediatric provider. Provider-adolescent communication may be lacking due to the overwhelming amount of expectations and topics that providers are expected to have knowledge of. It is possible that some of the topics expected to be covered by the providers may be discussed between parent and adolescent, hence relieving some of the burden on the health care provider. This study examines parent-adolescent communication during healthcare transition about three topics: sexual and reproductive health, autonomous medication/diet management, and genetics of the condition. Thirty individuals, including young adults who have completed transition and parents of young adults who completed transition, were included in the study. They were recruited from the Marfan syndrome, cystic fibrosis, and Phenylketonuria populations. Participants were asked to complete an online survey inquiring about the discussion of these topics between themselves and their adolescent or parent during the healthcare transition process. Sexual and reproductive health was discussed the least overall, and if medication/diet management was discussed individuals were more likely to have also discussed genetics $(p=0.009)$. The majority of participants felt that these discussions were or may have been beneficial to their transition period. Interestingly, the principal barrier identified in the prevention of these discussions was that individuals did not think to discuss the topic. As the study population is small, further studies are needed to enhance the generalizability of these results.

\title{
The Student Voice: Learner-Centered Changes in a Molecular Genetics Laboratory Rotation to Increase Student Satisfaction and Knowledge Integration
}

C. Guy ${ }^{1}$, A. Darden ${ }^{1}$

1. University of Oklahoma Health Science Center

Genetic Counseling is rapidly diversifying, with increasing numbers of genetic counselors specializing in industry or clinical laboratory positions. This, combined with the rapid pace of molecular technology development, puts increased emphasis on the importance of molecular genetics education in genetic counseling programs. While many genetic counseling programs have included molecular laboratory practicums as part of their educational structure, there is little published on the educational techniques and tools utilized in these rotations. In addition, the increased need for training in preparation for both understanding of molecular techniques and the role of a laboratory genetic counselor has resulted in an increased need for publication of educational tools and examination of the educational theories they are grounded in. The molecular laboratory rotation at the University of Oklahoma Masters in Genetic Counseling Program was restructured after student course evaluations revealed student dissatisfaction. Prior to the rotation, a laboratory genetic counselor was newly hired and was tasked with the objective of facilitating the molecular laboratory rotation. In consideration of possible educational interventions to respond to student feedback, several educational theories were explored to evaluate current practices in medical education in the consideration of application to genetic counseling education. Several tools and interventions were designed to increase the integration of knowledge and increase student satisfaction with the molecular laboratory practicum. These interventions included altering the timing of the Molecular Genetics Laboratory rotation to parallel the Molecular Genetics didactic course and to allow for a group rotation to promote social learning, such as described in social constructivism learning theory. A pre-rotation survey was given to assess the students' areas of interest. During the rotation, the student activities were coordinated by the laboratory genetic counselor and several activities were designed to promote experiential learning.

\section{The Conceptual and Practical Evolution of Education to Obtain Clinical Readiness in Genetic Counseling}

\section{S. Hassed ${ }^{1}$, S. Gillaspy ${ }^{1}$, N. Jacobs ${ }^{1}$}




\title{
1. University of Oklahoma Health Sciences Center
}

Counseling training programs have been active in modifying teaching strategies and implementing advances in methodology with the goal of improved experiences to prepare learners for clinical training. Nursing educators have recognized that providing students with all necessary content prior to graduation is no longer plausible and creating experiences that "replicate reality" result in improved student engagement and help students to "own the crucial content." Genetic Counseling Masters Programs frequently use some type of role-play component in practical clinical education, but there is no uniform expectation for ability prior to their first experiences with patients in a clinical setting. Some programs start future professionals in clinical rotations during their first semester of graduate education while others provide a didactic framework prior to beginning clinical rotations. However, for most students the opportunity for development of practical counseling skills are limited prior to clinical rotations, but role-play exercise has shown promise both during and after training. We describe development of the course "Introduction to Psychosocial Issues in Genetic Counseling" from its inception, "Version 1.0" in 2010, to "Version 2.5" in 2014 to improve student learning, empathic engagement with patients and families, and its place and coordination with other courses within the program. Specific changes relate to coordinated development between courses, genetic counseling skills practice, and addition of pediatric psychologists to the program. Skills practice includes family history construction, history taking, and synthesis of information. Changes in counseling instruction including basic counseling responses and reflective writing were instituted. Peer actor training vignettes with were created with planned incremental skill development based on use of the practice-based competencies including the opportunity to practice clinical supervision. Midterm and final evaluations were performed using trained "simulated patient" vignettes.

\section{MedGen: A Portal for Medical Genetics Information}

\author{
B. Kattman' ${ }^{1}$, A. Malheiro ${ }^{2}$, D. Maglott ${ }^{2}$, V. Gorelenkov², W. Shi ${ }^{2}$, F. Karmanov ${ }^{2}$, D. Hoffman ${ }^{2}$, G. Riley ${ }^{2}$, W. Rubinstein ${ }^{2}$ \\ 1. National Center for Biotechnology Information, National Institutes of Health \\ 2. National Center for Biotechnology InformationI / U.S. National Library of Medicine / National Institutes of Health
}

Data. Data. Data. In this day and age, we are bombarded by ever-increasing amounts of data. The challenge is to transform data into useful information and knowledge. Genetics professionals need to access high quality information about phenotypes to efficiently perform their many varied clinical tasks. MedGen (http://www.ncbi.nlm.nih.gov/medgen) is a freely available online resource that provides a gateway to information about human disorders and other phenotypes having a genetic component. MedGen is targeted to serve healthcare professionals and the medical genetics community by providing centralized access to diverse types of content related to phenotypes. Whether you are seeking to elucidate a differential diagnosis, locate a collection of curated reviews and professional guidelines, harmonize phenotype nomenclature or connect to available genetic testing and follow-up with a test result interpretation, MedGen is your starting point. MedGen uses the National Library of Medicine's Unified Medical Language System (UMLS) as a starting point to report and connect a variety of nomenclature vocabularies, including SNOMED CT, OMIM, HPO, and MeSH. Concept IDs are assigned to each concept and used to aggregate of the various identifiers from other vocabularies which enables mapping between the different nomenclature systems, and makes the use of genetic terms feasible in electronic medical records systems. MedGen also displays clinical summaries and definitions from sources such as GeneReviews, OMIM and Genetics Home Reference. Using the PubMed database as support, MedGen is able to offer users easy access to review articles, curated suggested reading and professional guidelines. MedGen also connects to a plethora of associated content, including available genetic testing via the Genetic Testing Registry, consumer resources, and molecular resources such as ClinVar which provides information on the clinical significance of human variation. This presentation will introduce the audience to MedGen, displaying the features relevant to the medical genetics community.

\section{Mapping and Evaluation of a Genetic Counseling Training Program Curriculum}

\section{T. Lepard Tassin ${ }^{1}$, L. Williamson Dean ${ }^{1}$}

\section{University of Arkansas for Medical Sciences}

Mapping and evaluation of curricula improves student learning outcomes and helps programs meet requirements for accreditation. The purpose of this project was to develop an approach to curriculum mapping of didactic coursework for a genetic counseling program. The curriculum mapping plan was based on the program requirement elements outlined by the American Board of Genetic Counseling (ABGC). The project addressed seven aims: (1) determine if course syllabi provide a clear description of the learning objective, requirements, and criteria for grading and evaluation; (2) ensure objective measures for evaluating students' progress in courses; (3) ensure documentation of learning experiences, including instructional material and presentations; (4) determine if course sequence allows students to develop the competencies necessary for graduation and that subject matter increases in complexity; (5) ensure that the ABGC content areas are being covered sufficiently; (6) ensure teaching resources are not duplicated; and (7) identify areas for quality improvement. Four data collection tools were developed (two faculty surveys, syllabi review, and student interview questionnaire). Results for year 1 of the program show that of the 32 ABGC content areas that programs are required to cover, 31 were covered, and instructors formally assessed a student's knowledge acquisition 3.77 times. A diverse range of instructional material was used (35 different resources). Syllabi were reviewed for 10 critical items. All items were included in $50 \%$ of the syllabi in Fall 2012, and $62.5 \%$ in Spring 2013. However, of the content areas taught in each course, only $37 \%$ of these content areas were directly expressed in the syllabi in Fall 2012 and $38 \%$ in Spring 2013. The mapping process has benefited the program by providing a visual representation of the curriculum, noting overlapping coverage of topics, identifying and addressing gaps in the curriculum, improving syllabi, and promoting collaboration among faculty.

Twitter Activity before and after Association for Molecular Pathology v. Myriad Genetics, Inc. Supreme Court Decision Using NodeXL

\author{
A. Lewis ${ }^{1}$, M. Riddell ${ }^{2}$, J. Holsinger ${ }^{2}$ \\ 1. University of Kentucky \\ 2. University of Kentucky College of Public Health
}


Social media has become a standard way of communicating information among individuals and organizations. In 2013, several significant events occurred related to cancer genetics including the June 13, 2013 decision by the U.S. Supreme Court on the Association for Molecular Pathology, et al. v. Myriad Genetics, Inc., et al. case. The purpose of this study was to determine if posts to Twitter about Myriad Genetics were different before and after the Supreme Court Decision. We hypothesize that tweets increased showing that social media can be an effective tool in increasing public awareness of genetics after a high-level policy decision. Data on tweets containing the search phrase "Myriad Genetics" was collected on June 10, 2013 (TW1) and on June 20, 2013 (TW2) using NodeXL version 1.0.1.238. NodeXL is a free, open-source extension for Microsoft Excel available for public download that enables users to easily search, collect and visualize social network data. Data from a total of 8 days prior and 8 days after the Supreme Court decision was analyzed using NodeXL tools to determine differences in Tweets for these two time points. Results showed that prior to the Supreme Court decision, a total of 166 tweets occurred containing the search term compared to 1615 tweets after the Supreme Court decision, representing a $973 \%$ increase after this major news event. A total of 103 unique websites were linked in $79.1 \%$ of tweets in TW1 and 953 unique websites in $77.4 \%$ of tweets in TW2. Both of these websites were articles about the pending/recent Supreme Court Decision. The most frequent TW1 hashtags were \#indeed, \#jobs, \#monopolies, \#can with each appearing 8 times. In TW2, the most frequent hashtags were \#scotus (58 occurrences) and \#myriad ( 35 occurrences). The results of this study suggest that social media can be an important medium through which the public discusses and discovers information about genetics. These results can be used to develop and distribute targeted messages about genetics to the public for educational and informational purposes through social media.

\title{
The Impact of Rosa's Law on Describing Persons with Intellectual Disability
}

\author{
A. Lutter ${ }^{1}$, S. DeBrosse ${ }^{2}$, D. Culler ${ }^{3}$, A. Matthews ${ }^{1}$ \\ 1. Case Western Reserve University \\ 2. Department of Pediatrics, Case Western Reserve University School of Medicine \\ 3. University Hospitals of Cleveland
}

Terminology used to describe individuals with Intellectual Disability (ID) is confusing as numerous terms are used to describe this diagnosis. This study explored if Rosa's law, which states that "intellectual disability" should replace "mental retardation" in federal documentation, impacted terminology preferred by members of the National Society of Genetic Counselors $(n=310)$ and parents of individuals with ID from the Genetic Alliance $(n=88)$. Online survey results showed a significant difference between genetic counselors and parents $(p<0.001)$ in their most preferred terminology. Genetic counselors preferred intellectual disability $(66.3 \%)$ followed by mental retardation (31.6\%) $(n=267)$. No parent chose mental retardation as their one preferred term $(n=72)$, however, $85 \%$ of parents stated that mental retardation was their least preferred term. Parents preferred developmentally delayed $(40.3 \%)$ followed by developmental disability $(27.8 \%$ ). Although genetic counselors chose ID as their most preferred term, only $30 \%$ reported awareness of Rosa's law. Interestingly, of the 31 parents who reported awareness of Rosa's law, only $13 \%$ preferred ID. No correlation between awareness of Rosa's law and preferred terminology was found. Of those counselors practicing for less than 4 years $(n=151)$, approximately $69 \%$ chose the term intellectual disability as their most preferred term. There was a statistical difference between most preferred term of counselors who worked 4 years or less and those genetic counselors who had been working for 5 or more years $(p<0.001)$. This may indicate an "indirect" impact of Rosa's law on preferred terminology of genetic counselors; that is, counselors may have heard the term intellectual disability being used more often because of Rosa's law, even if they are unaware that the term intellectual disability has been federally mandated. While there was no consensus as to which term best described individuals with ID, identifying a unifying term may be beneficial in preventing confusion and clarifying diagnoses.

\section{Professional, Ethical and Legal Issues of Genetic Testing and Personal Insurance}

\section{Ngueng Feze', M. Lane², Y. Joly ${ }^{1}$}

1. McGill University Centre of Genomics and Policy

2. IWK Health Centre, Maritime Medical Genetics Service

Introduction: Genetic discrimination (GD) in the context of insurance has been a concern for more than three decades. Since the adoption of GINA and the emission of NSGC cancer practice guidelines, genetic counselors have been advised to inform patients about the possibility of GD and the limitations of applicable laws. Purpose: This study is the first to account for the experiences of Canadian cancer genetics counseling on genetics and personal insurance in a context where there is no specific legal protection against GD. Methods: A semi-structured questionnaire containing a combination of open-ended and multiple choice items was formulated following a review of the genetic counseling literature on genetics and insurance. The 2003 Pfeffer et al. study was particularly helpful in designing the instrument. Participants were recruited via the Canadian Association of Genetic Counselors (CAGC) membership listserv. The 36 eligible participants were CAGC members practicing in Canada and working at least part-time in cancer genetics. Qualitative data was tallied and graphed, however formal statistical analyses were not conducted due to the small sample size. Results: All respondents reported discussing the issue of genetics and personal insurance often at the patient's request. Several factors influenced the content, depth and length of these discussions including age, cancer status, existence of siblings or children, and insurance needs. Counselors disclosed not having sufficient training on this subject and expressed a need for additional information and resources for themselves and their patients. Conclusions: While genetics and personal insurance are commonly discussed in Canadian cancer genetic counseling sessions, the information provided by counselors about patient's duty to disclose information related to their insurability to the insurers was often incomplete, inaccurate or could be misinterpreted by patients, underlining further the need for additional resources on the ethical and legal implications of genetics and personal insurance.

\section{Implementation of Crisis Intervention Training in Genetic Counseling Training Programs}

R. Reese $^{1}$, C. Hurst ${ }^{1}$, F. Brewer ${ }^{1}$

1. The University of Alabama at Birmingham 
Crises are situations characterized by disruption of an individual's coping mechanisms. Crises in medical settings, including genetic diagnoses, may lead to harmful consequences including depression and suicide. Genetic disease may have profound effects on both the affected individual and their family due to their chronic and often incurable nature and impact on family planning. Genetic counselors are healthcare providers who communicate genetic risk information and provide support. Although crisis intervention training (CIT) is a required component of genetic counseling training program curricula and competencies per the Accreditation Council for Genetic Counseling, there are no standardized guidelines to date providing suggestions for the delivery method and content of this training. This survey-based study was designed to assess practicing genetic counselors' experience with CIT and patients in crisis and to provide recommendations for training programs' consideration when developing CIT curricula. The majority of participants (88 \%) supported the sentiment that CIT is an important part of genetic counseling training. Over $79 \%$ of participants did not receive formal CIT during their graduate school training. Counselors working in prenatal and laboratory specialties reported having patients in crisis more often than other specialties. Thematic analysis revealed that many participants desired more in-depth crisis training. Preferences included how to recognize individuals in crisis or at suicide risk, how to calm or diffuse a patient, how to develop a suicide safety plan, and how to navigate the referral network within their institution. The majority of participants felt that genetic counseling patients were not being adequately referred for long-term mental health counseling. The majority of CIT supporters identified a face-to-face class or simulation as the most desired delivery method. The results from this study discovered gaps in genetic counselors' crisis training background and identified elements recommended to be included in future curricula.

Disability Experiences and Perspectives regarding Reproductive Decisions, Parenting and the Utility of Genetic Services: A Qualitative Study C. Roadhouse ${ }^{1,2}$, C. Shuman ${ }^{2}$, K. Anstey ${ }^{3}$, K. Sappleton ${ }^{2}$, D. Chitayat ${ }^{2,4}$, E. Ignagni ${ }^{5}$

1. University of Toronto

2. The Hospital for Sick Children

3. University Health Network

4. Mount Sinai Hospital

5. Ryerson University School of Disability Studies

Introduction: Genetic counselors adopt seemingly contradictory roles: supporting individuals with genetic conditions and offering prenatal testing and termination to avoid a child with a disability. This contributes to the tension between the disability and clinical genetics communities. Varying opinions exist among the disability community: some value genetic services while others are opposed. However, there is limited research exploring the opinions of individuals with a disability regarding issues related to reproduction and genetic services in the context of unique personal experience. Purpose: This study sought to gain the perspectives of individuals who self-identify as having a non-acquired disability and investigate their experiences, thoughts about reproduction and parenting, and perceptions of genetic services. Methods: We utilized a qualitative exploratory study design, specifically the Interpretive Description methodology. Men and women who self-identified as having a disability were recruited through disability support services in Ontario. Personal experience with genetic counseling did not affect inclusion. Ten semi-structured interviews were conducted in person and via telephone. Transcripts were analyzed thematically using qualitative content analysis. Results: Preliminary analysis indicates the following themes: (i) social construct of disability impacts personal experience and reproductive decisions; (ii) importance of self-advocacy within the health care system and community: room for provider education; (iii) value of accurate information and personal choice surrounding reproduction: an appreciation for genetic services; (iv) normalization of pregnancy and parenting issues. Conclusions: These findings contribute to our understanding of the disability perspective toward reproductive decision making and genetic services. This research also serves to promote a meaningful dialogue between genetic counselors and the disability community, and has the potential to enhance the genetic and reproductive care provided to individuals with disabilities.

\section{Knowledge of Sickle Cell Disease in Ghana}

D. Schlegel ${ }^{1}$, A. Campbell ${ }^{1}$, M. Gornick ${ }^{1}$, K. Gyan ${ }^{1}$, P. Midathada ${ }^{1}$, B. Yashar ${ }^{1}$

\section{University of Michigan}

Introduction: Sickle cell disease (SCD) is a blood disorder in which affected blood cells take on a "sickle" shape, causing blockages in blood vessels and giving rise to pain crises and organ damage. In Ghana, $2 \%$ of the population has SCD, and 25-30\% has SCT (carriers). However, the level of knowledge about SCD in Ghana has been relatively unexplored. Purpose: The aim of this study was to conduct a needs assessment of the Ghanaian population with respect to knowledge of SCD/SCT to help inform the design of future health interventions. The objectives were to identify the level of knowledge about $\mathrm{SCD} / \mathrm{SCT}$, the types of information about SCD/SCT that Ghanaians have interest in receiving, and the differences in types of knowledge possessed by females and by males. Methods: 104 Ghanaian adults were randomly recruited from the general, non-SCD clinic at Korle-Bu Teaching Hospital in Accra, Ghana. They completed in-person surveys, which were pilot tested in the first 14 participants, about their knowledge of SCD and its inheritance. SPSS was used to calculate variable frequencies and to run the "derived knowledge" variable against various subject demographics. Results: In general, participants were aware of SCD inheritance. $98.9 \%$ had heard of SCD, $75.6 \%$ had heard of SCT, $76.7 \%$ said that SCD "runs in the family," and $91.1 \%$ said that SCD is inherited from both parents. However, many people were not aware of the SCD status of their family members, and only $26.7 \%$ of the participants had undergone genetic testing. Knowledge was found to differ significantly by sex, with women having a significantly higher knowledge score than men $(p=0.005)$, especially at the lowest level of education $(p=0.009)$ and at the lowest $(p=0.006)$ and middle levels $(p=0.041)$ of socioeconomic status. Conclusions: Our study suggests that the most useful interventions for this population would have an emphasis on access to and knowledge about testing and about communication with family members about their SCD status. The sources of women's knowledge would be a useful focus of future investigation.

\section{Hereditary Breast and Ovarian Cancer Education Needs of Georgia Primary Care Residency Training Programs}

\section{E. Schmitt ${ }^{1}$, M. Martin ${ }^{2}$, D. Meaney-Delman ${ }^{3}$, C. Bellcross ${ }^{4}$}


1. Emory University

2. Georgia Center for Oncology Research and Education

3. Department of Gynecology and Obstetrics, Emory University School of Medicine

4. Emory University School of Medicine

Introduction: Despite strong evidence supporting the benefits of genetic risk assessment and testing, many families with Hereditary Breast and Ovarian Cancer syndrome (HBOC) are not identified. Primary care providers (PCPs) are at the forefront of caring for patients that may be affected by HBOC. Current medical training may not provide practicing PCPs with the necessary knowledge to identify families at high-risk for HBOC. The purpose of this study is to assess the HBOC educational needs specific to Georgia primary care residency training programs. Methods: Surveys were distributed to individuals from 22 primary care residencies. Program faculty and residents of family medicine, obstetrics and gynecology, and internal medicine programs in Georgia were invited to participate. Results: Respondents include 114 residents and 10 faculty members. Of residents, $57 \%$ do not recall having formal HBOC training. A striking percentage fail to recognize paternal inheritance of HBOC (31\%), incorrectly apply autosomal dominant inheritance (51\%), and incorrectly identify colon (28\%) and uterine cancer (18\%) as HBOC-associated diagnoses. Residents are interested in learning more about HBOC (95\%) and prefer to do so through case-based, interactive lectures. Specific residency curricula deficits indicated in responses from faculty members reveal a lack of emphasis on HBOC inheritance, identification of high-risk histories, paternal inheritance, and use of referral tools. Conclusions: Opportunities for educational interventions to improve HBOC knowledge at the primary care residency level are apparent. Targeted education modules are essential to improve future PCP ability to identify families at risk for HBOC and maximize the benefits of genetic risk assessment and prevention strategies.

Patient and Family Outreach for Marfan Syndrome and Related Disorders: Findings from a Multidisciplinary Education Day

\author{
A. Shikany ${ }^{1}$, J. Buffone ${ }^{2}$, E. Miller ${ }^{1}$, A. Parrott ${ }^{1}$, R. Hinton ${ }^{1}$ \\ 1. Cincinnati Children's Hospital Medical Center \\ 2. The Marfan Foundation
}

Marfan syndrome (MFS) is a pleiotropic autosomal dominant disorder with a prevalence of 1 in 5,000. Management for patients with MFS is complex, involving multiple subspecialties. While education is typically provided by the medical team, including genetic counselors, little is known about the comfort level with disease knowledge, or the educational needs of patients and families with MFS. Importantly, practice variation is common for this patient population, which impacts patient and family knowledge. The Cardiovascular Genetics Program in partnership with the Marfan Foundation (MF) sought to increase patient and family awareness by hosting a MFS and Related Disorders Education Day. This one-day conference included 8 presentations given by experts in MFS. Paired pre- and post-conference surveys were given to 37 conference participants anonymously to assess patient and family-reported disease knowledge and educational needs. Twenty-eight participants $(76 \%)$ completed both the pre- and post-conference surveys. Sixty-four percent $(n=$ $18)$ and $21 \%(n=6)$ identified the medical team and the Marfan Foundation as primary information sources, respectively. Seven respondents were affected, while remaining respondents were unaffected but had an affected relative. All affected participants reported they were most interested in the background and history of MFS, while unaffected participants, most of whom were parents or grandparents, expressed interest in transitioning care from adolescence to adulthood. The majority of participants (89\%) reported an increase in their ability to advocate and manage care for themselves or their affected family member, and $85 \%$ of participants indicated that they would attend a similar education day in the future. These data suggest that a disease-specific education day is an effective outreach tool for increasing disease knowledge and suggests a standardized approach to education with the involvement of diseasespecific, resource-rich organizations, such as the MF may increase ownership of condition and ultimately improve care.

\title{
Group Prenatal Genetic Counseling Facilitates Patient Decision-Making
}

\section{E. Sturm ${ }^{1}$, K. Hickey ${ }^{1}$}

\section{Walter Reed National Military Medical Center}

The American College of OB/GYN has recommended that options for prenatal testing for aneuploidy should be available to and discussed with all women, regardless of age. Coupled with the growing demands of genetic counseling are expanding test menus and rapidly evolving genetic testing options often difficult for non-genetic healthcare providers to navigate and utilize appropriately. We sought to examine outcomes of group prenatal genetic counseling by assessing a patient's level of uncertainty or confidence in making decisions about prenatal genetic testing. Patients who attended a standard group obstetric orientation class were offered to participate. Participants were given surveys to complete immediately before and after attending group prenatal genetic counseling class facilitated by a genetic counselor. The 10-item Decisional Conflict Scale was administered both before and after the class, and the four-item SURE decisional conflict screen was completed afterward. Additional feedback was elicited in open-ended questions asking participants to describe how they thought the class could be improved and what they liked or disliked about the class. 168 surveys were returned. Participants demonstrated significantly lower decisional conflict after attending the group class. The number of patients who felt they had enough information to make a decision increased from 54 before attending the class to 141 afterward $(p<.001)$. The number of participants who were undecided about prenatal testing decreased from 101 before to 31 afterward $(p<.001)$. The number of patients who felt sure about their choice increased from 42 before to 123 afterward $(p<.001)$. These findings suggest that group genetic counseling is effective in the prenatal setting in providing a greater number of women in pregnancy information about prenatal genetic testing options, including the benefits, limitations, risks and outcomes to allow them to make decisions that are consistent with their values and for which they feel respected, supported and satisfied.

\section{"It Really Permeates Your Consciousness.” Examining the Impact of Genetic Counselling Training on Students' Prenatal Risk Perception and Reproductive Decision Making}

I. Thompson ${ }^{1}$, J. Austin ${ }^{1}$, A. Semaka ${ }^{1}$ 


\section{University of British Columbia}

Increased maternal anxiety in the preconception and prenatal period amongst genetic counselors is a previously described phenomenon. Knowledge of the frequency of genetic abnormalities, potential pregnancy complications, and prenatal screening and testing options provides genetic counselors with a unique perspective on potential risks involved in pregnancy and childbearing. The origins of this maternal anxiety have yet to be determined; however it is possible that such concerns begin during training, when students are faced with the realities of prenatal risks for the first time. The purpose of this qualitative study was to explore how the first semester of genetic counseling training programs influenced students' perceptions of prenatal risk, and whether and how this affected their reproductive decision-making. We also sought to explore the psychological impact of changes in risk perception and to assess students' needs for support surrounding these issues. Methods: Interviews were conducted with 8 Canadian genetic counseling students, prior to the training program and after the first semester of study. Line-by-line and focused coding were used for analysis, along with the constant comparative method. Results: Students reported an overwhelming increase in thought frequency regarding prenatal risks, and described becoming more focused on, and fearful of, maternal agerelated risks. Students who attempted to make personal meaning of new prenatal risk information especially experienced an increase in anxiety when thinking about having children, while students who were more objective when incorporating new risk information did not experience a psychological impact. Conclusion: This is the first and only study to explore prenatal risk perception amongst genetic counseling students. We found that students experienced an increased risk perception, which changed their stances on decisions they plan to make during pregnancy. This study points to the importance of future studies to further elucidate the origins of the prenatal maternal anxiety amongst genetic counselors

\section{Parental Satisfaction and Teacher Perspectives on Inclusive Education of Students with Asperger Syndrome: An Educational Tool}

\section{H. Warren ${ }^{1}$, R. Ferrante ${ }^{2}$, D. Zvejnieks ${ }^{3}$, R. Hock ${ }^{4}$}

1. University of South Carolina School of Medicine

2. Department of Pediatrics, University of South Carolina School of Medicine

3. University of South Carolina

4. College of Social Work, University of South Carolina

Introduction: The number of individuals diagnosed with autism spectrum disorder (ASD) is continuously increasing. Students diagnosed with Asperger syndrome (AS) or high-functioning autism (HFA) are frequently enrolled in regular education classrooms, although social difficulties create challenges. Little research has evaluated parental satisfaction and teachers' desired knowledge regarding students with AS in secondary schools. Purpose: The goal of the study was to develop an educational tool to aid in initiating a parent-teacher relationship for a student with AS or HFA. The tool is an added resource for pediatric genetic counselors who frequently see individuals with autism. Methods: Parents were invited through local and social media support groups to complete an anonymous online survey that included questions measuring anxiety and satisfaction regarding their child's education, in addition to demographic information. Teachers were invited from high schools in South Carolina by receiving the invitation letter from their school principals and also through an educational conference in Columbia, SC. The teacher survey was completed in interview form and focused on experiences teaching students with AS, desired parental involvement, and desired knowledge about the diagnosis. Results: Of the total respondents $(N=172), 101$ parent participants met the inclusion criteria. Their anxiety significantly increased when considering their child's education $(p<.001)$. Overall, parents were satisfied with teacher qualities, but there was dissatisfaction regarding the education experience as a whole. Teacher participants $(N=10)$ desired knowledge including strategies for handling situations that may arise and possible triggers. Conclusions: Teachers expressed interest in parent contact, yet a noticeable subset of parent respondents (37\%) found it difficult to communicate with their child's teachers. Based on the findings of this study, the educational tool can assist in initiating communication between parents of a child with AS or HFA and their child's teachers.

\section{Form Follows Function: Development of a Model for Clinical Supervision Practice in Genetic Counseling}

\section{Wherley ${ }^{1}$, P. McCarthy-Veach ${ }^{1}$, B. Hatten ${ }^{2}$, B. LeRoy ${ }^{3}$}

1. University of Minnesota

2. Abbott Northwestern Hospital

3. Deptartment of Genetics, Cell Biology and Development, University of Minnesota

Supervision plays a vital role in genetic counselor training. The Accreditation Counseling for Genetic Counseling Practiced-Based Competencies require genetic counselors to understand supervision processes, and one study by Eubanks-Higgins et al. identified genetic counseling supervisor competencies. To date, however, models describing genetic counseling supervision processes and outcomes are lacking. We developed a supervision model specific to clinical supervision of genetic counseling students. The model provides a framework to promote comprehensive and consistent supervision training for genetic counselors. Using the principle "form follows function" we designed the model to reflect and reinforce McCarthy Veach et al.'s empirically derived model of genetic counseling practice — the "Reciprocal Engagement Model" (REM). The REM has mutually interactive educational, relational and psychosocial components. The Reciprocal Engagement Model of Supervision (REM-S) contains tenets, goals and outcomes reminiscent of those in the REM. REM-S tenets are: genetic counseling services are key; relationship is integral to genetic counseling supervision; and student autonomy must be supported. Outcomes are: student understands and applies information to practice effectively, engage in self-reflective and independent practice, and develop professionally. REM-S goals are informed by the REM and a critical review of literature on clinical supervision in genetic counseling. A search of four electronic databases identified 12 published studies, 1 chapter, and 55 commentaries or personal essays on the process and impact of genetic counseling supervision. We also reviewed articles in medicine and psychology to confirm the REM-S has supervision elements common in healthcare while remaining unique to genetic counseling. The REM-S shows promise for enriching genetic counselor supervision training and practice, and promoting research on clinical supervision. The REM-S is presented in detail along with specific examples and training and research suggestions. 
Traditional Textbook Photographs Negatively Impact Student Perception of Individuals with Visible Genetic Conditions

\author{
N. Wood ${ }^{1}$, C. Hornbuckle ${ }^{2}$, R. Guidotti ${ }^{3}$, L. Nicely ${ }^{4}$, S. Elsea ${ }^{5}$ \\ 1. Baystate Health \\ 2. Department of Genetics and Metabolism, Children's Medical Center Dallas \\ 3. Positive Exposure \\ 4. Virginia Commonwealth Univesity, Medical College of Virginia \\ 5. Department of Human and Molecular Genetics, Baylor College of Medicine
}

Purpose: To determine if natural portraits in textbooks affect learning or perception of individuals with visible genetic conditions compared to traditional textbook photographs. Methods: Students $(n=516)$ from a variety of medical and allied health care programs were randomly assigned to online surveys modeled after textbook entries on Marfan syndrome, Cornelia de Lange syndrome, and Noonan syndrome that included either traditional textbook photographs (survey A) or natural portraits (survey B). Participants' comprehension and perception of pictured individuals were assessed using multiple choice, 5-point Likert scale, and open responses. Results: Comprehension analysis of the presented information revealed no significant differences between groups, indicating that learning was not impacted by the photograph. However, analysis of perception measures revealed that students who took survey B generally rated the individuals more positively on the Likert scale than survey A. Significant differences $(p<0.03)$ between groups were seen in mean perception scores for all three genetic conditions with the most notable differences observed in Cornelia de Lange and Noonan syndromes. Interestingly, the option to select "Prefer Not to Answer" (PNA) in place of selecting a Likert value significantly impacted the mean perception score. A markedly increased frequency of PNA was seen in all categories for survey B $(20.3-28.8 \%, n=266)$ compared to survey A $(11.8-18.1 \%, n=250)$, implying that the natural portraits elicited a hesitation to judge the individual based solely on their picture and diagnosis and this was supported by open responses that stated this explicitly. Conclusion: Natural portraits do not hinder the reader's comprehension, positively influence the perception of the affected individual, and may reduce willingness to negatively judge the person. Thus, use of natural portraits for education reinforces the human side of genetic disorders, while traditional textbook photographs may reinforce negative perceptions of persons with visible genetic conditions.

\title{
GC Professional Roles
}

\section{The Importance of Suicide Screening in the Genetic Counseling Setting}

\section{Anderson ${ }^{1}$, B. Biesecker², L. Horowitz ${ }^{3}$}

1. National Human Genome Research Institute, Johns Hopkins University

2. Genetic Counseling Training Program, Johns Hopkins University/National Human Genome Research Institute

3. National Institute of Mental Health, National Institutes of Health

Suicide is an international public health crisis and the second leading cause of death for youth in the United States. Poor physical health is a risk factor for suicidal thoughts and behaviors in this population. Young people utilizing genetic counseling services are often experiencing stress from chronic ill health and family health concerns. This population is potentially at elevated risk and therefore may benefit from suicide risk screening. We aim to demonstrate the necessity of suicide screening in genetics clinics and to describe a screening tool that can be implemented by genetic counselors. This presentation highlights research on at-risk populations encountered in genetic counseling. We present the Ask Suicide-Screening Questions (ASQ), a four-item screening tool for identification of pediatric patients at risk for suicide. The ASQ has been shown to have high sensitivity and specificity in youth with chronic and acute illnesses presenting to emergency rooms (ER). The ASQ can be administered rapidly by non-psychiatric clinicians in less than 2 minutes. Most parents of ER patients found suicide risk screening acceptable and nearly all participating-patients valued the importance of screening youth for suicide risk. Genetic counselors are uniquely positioned to screen clients for mental health concerns, including suicide; yet suicide screening is not routine practice and clients at risk may go unrecognized. This brief screening tool could help genetic counselors, who are on the forefront of this critical public health threat, to identify and refer clients at-risk for suicide. Suicide screening is congruent with the National Society of Genetic Counselors ethics code of caring for the overall welfare of clients. Training in the use of suicide screening tools, such as the ASQ, can be incorporated into genetic counseling graduate programs and taught at the Annual Education Conference. Routine suicide screening should be standard practice, especially in pediatric genetics.

\section{Current Practices and Perceptions of Ophthalmic Genetic Counselors}

\section{S. H. Chen ${ }^{1}$, C. Guy ${ }^{1}$, S. Hassed ${ }^{1}$, T. Yanovitch ${ }^{2}$}

\section{University of Oklahoma Health Sciences Center \\ 2. Dean McGee Eye Institute}

Ophthalmic genetic counseling is a relatively novel sub-specialty that bridges genetics and ophthalmology and contributes to patient care in both fields. To date, there are few publications that describe ophthalmic genetic counseling and its delivery model, clinical utility, current practices, and future direction. A total of 66 ophthalmologists from the American Association for Pediatric Ophthalmology and Strabismus and 22 ophthalmic genetic counselors responded to an anonymous online survey. Common themes were identified using thematic analysis, and significant correlations were confirmed using two-tailed fisher exact tests with a standard $p$-value of 0.05 . Of all respondents, $88 \%$ agreed that ophthalmic genetic counseling is beneficial for patients. Clinical roles include assisting with diagnostic workups, diagnosis counseling, genetic testing, and research coordination. Inadequate ophthalmic knowledge and the inability to perform physical exams and make diagnoses were cited as significant limitations for ophthalmic genetic counselors. Both ophthalmologists and genetic counselors noted cost justification, duplication of genetic services, and a lack of understanding about genetic counseling as challenges that may impede the establishment of genetic counselors within ophthalmology clinics. More than $93 \%$ of 
respondents were satisfied with the overall care that patients received from ophthalmic genetic counseling, although our study identified areas for improvement, including communication between genetic providers and ophthalmologists and the sharing and accessibility of genetic resources and services. This study describes the current perceptions and practices of ophthalmic genetic counseling and identifies limitations and gaps in patient care that should be addressed in the future direction of this field.

\title{
Clinical Exome Sequencing Test Development: Roles for Laboratory Genetic Counselors
}

E. H. Denenberg ${ }^{1}$, K. McDonald Gibson ${ }^{1}$, K. Cao $^{1}$, Z. Yu ${ }^{1}$, A. M. I. Nesbitt ${ }^{1}$, T. Tischler ${ }^{1}$, B. McLarney ${ }^{1}$, S. Mulchandani ${ }^{1}$, E. T. Dechene ${ }^{1}$, N. B. Spinner ${ }^{1}$, A. Santani ${ }^{1}$

\section{Children's Hospital of Philadelphia}

Within the Division of Genomic Diagnostics (DGD) a team of laboratory scientists, post doctoral fellows, directors, and laboratory genetic counselors (GCs) launched clinical exome sequencing (ES). The group had three major goals: 1) create a standardized workflow for data interpretation, 2) customize a laboratory information management system (LIMS) for ES data management, and 3) perform studies to validate ES. The GCs contributed to the accomplishment of each goal. The first goal of standardizing data interpretation workflow led to the involvement of GCs with phenotype capture. Medical records and test requisition forms were reviewed by GCs, who stored relevant phenotypic information in the LIMS. In addition, GCs were instrumental in creating the test requisition and consent forms. The standardized data interpretation workflow led to data analysis being performed by multiple members of the clinical exome team whereas reports were written by the GCs and directors. The second goal, to customize the LIMS for ES, was also aided by GC input. The software was designed to store information important for compliance, such as quality control metrics, software and database versions, turn-around-time, and evidence utilized to identify likely disease-causing variants. Phenotype capture forms, report templates, and canned text were also built into the LIMS. Finally the third goal, validation of ES, was achieved using the College of American Pathologists Next Generation Sequencing guidelines. Using this approach, ES demonstrated high sensitivity, specificity, and reproducibility. To further validate ES, specimens previously analyzed at a reference laboratory were reanalyzed by the clinical exome team with a high concordance of results. In addition to GCs assisting with the accomplishment of ES test development goals, they also made recommendations on billing and reimbursement strategies. Within the clinical genomics laboratory, GCs are specially trained professionals uniquely positioned to have a large impact on the development and implementation of novel genomic tests.

\section{U.S. Preventive Services Task Force and Genetic Counseling for BRCA Mutations: Tools to Help Your Primary Care Colleagues}

\author{
M. Doerr ${ }^{1}$, E. Gabitzsch ${ }^{2}$, D. Smith ${ }^{2}$, K. Teng ${ }^{2}$, C. Eng ${ }^{2}$ \\ 1. Cleveland Clinic Foundation \\ 2. Cleveland Clinic
}

The U.S. Preventive Services Task Force (USPSTF) is an independent panel of private-sector experts in primary care and prevention overseen by the US Department of Health and Human Services' Agency for Healthcare Research and Quality. Under the Affordable Care Act (ACA) the Centers for Medicare and Medicaid Services (CMS) are required to cover preventive care recommended by USPSTF (grade A and B recommendations). In December 2013, USPSTF published Risk Assessment, Genetic Counseling, and Genetic Testing for BRCA-Related Cancer in Women to provide guidance on BRCA assessment for asymptomatic women without a personal history of a BRCA-related cancer. USPSTF gives a grade B recommendation that primary care providers screen women who have family members with breast, ovarian, tubal, or peritoneal cancer with one of several screening tools designed to identify a family history that may be associated with $B R C A 1 / 2$ mutations. According to the recommendation, women with positive screening results should receive genetic counseling and, if indicated after counseling, BRCA testing. USPSTF gave a grade D (recommended against) genetic counseling for women whose family history is "not associated" with an increased risk for BRCA mutations. Assessment of men at risk of BRCA mutation is not addressed by the statement. Genetic counselors should be prepared to support primary care clinicians in understanding the benefits and limitations of applying the USPSTF recommendation statement to their patient panels. Screening tools listed in the recommendation include Ontario Family History Assessment Tool, Manchester Scoring System, Referral Screening Tool, Pedigree Assessment Tool, and FHS-7. Based on the strengths and limitations of each model, we have developed an algorithm to assist with identifying the best fit tool for your colleague's primary care practice needs, e.g., time for tool completion. Use of this algorithm facilitates systematic implementation of the chosen risk assessment, including identifying possible health information technology integration points.

\section{Incorporating Computer-Aided Facial Analysis Software into Genetic Counseling Practice \\ D. Gelbman ${ }^{1}$, V. Weinblatt ${ }^{2}$}

\section{FDNA \\ 2. Integrated Genetics}

In pediatric genetics settings, the genetic counselor is often the primary caregiver, assigned with the complex task of synthesizing relevant patient data and facilitating a clinical evaluation plan which ideally leads to a definitive diagnosis and patient care plan. It is also the genetic counselor that often manages the medical records, facilitates the ordering of laboratory tests and communicates the evaluation and management plan to the families and referring physicians. Creating this plan with a differential diagnosis is a time consuming and labor intensive process, requiring tasks such as a comprehensive review of medical literature, analysis of relevant test offerings and consultation with other experts. This process, albeit critical for comprehensive patient care, can contribute to long waiting times for a patient to be seen for a genetics evaluation, delaying associated evaluations and treatments. Face2Gene, a new search and reference mobile application, created to aid and empower clinicians in accelerating and increasing confidence in the research and investigation of dysmorphic genetic syndromes, can streamline this process and support genetic counselors in their evaluation. 
Face2Gene incorporates an extensively validated unique facial analysis technology, developed by leveraging thousands of years of combined experience from hundreds of dysmorphologists. This HIPAA compliant software facilitates automatic detection of dysmorphic features and recognizable patterns of human malformations from a facial photograph, which is filtered through an advanced search engine, offering access to multiple medical references for an elaborate, yet focused review. Results may be compiled into an editable report, which may be used to communicate findings to peers or families. Wide adoption of this utility by genetic counselors in clinical settings can augment their pivotal role in the diagnosis and management of patients, reduce their work load and enable them to assume a more active role in medical staff training and research activities.

\title{
The Relationship between Burnout and Occupational Stress in Genetic Counselors
}

\author{
B. Johnstone ${ }^{1,}$, A. Kaiser ${ }^{1}$, M. Injeyan ${ }^{2}$, K. Sappleton ${ }^{3}$, D. Chitayat ${ }^{3}$, C. Shuman ${ }^{1,3}$ \\ 1. University of Toronto \\ 2. Mount Sinai Hospital \\ 3. The Hospital for Sick Children
}

Introduction: Burnout has been described as a wearing out due to excessive demands and studies show it has a significant impact on work life. Nursing research has shown that relationships between occupational stress and burnout can be used to suggest intervention recommendations. While burnout is documented in genetic counselors (GCs), there is limited research exploring the causes of GC specific burnout. Hypothesis: Burnout, as experienced by GCs, is positively associated with occupational stress. Methods: We recruited participants though the NSGC, Canadian Association of Genetic Counsellors, and Australasian Society of Genetic Counsellors listservs. Respondents $(n=280)$ completed an online questionnaire containing the Maslach Burnout Inventory, to measure burnout on three subscales (cynicism, exhaustion, and professional efficacy); the Occupational Stress Inventory, to assess aspects of occupational stress; and demographic questions. Data was analyzed using descriptive statistics, Pearson correlations, and multiple linear regressions. Results: Preliminary analysis shows $48 \%$ of respondents indicate they feel burned out from their work a few times a month or more. Pearson correlations indicate aspects of occupational stress correlated to cynicism include role insufficiency $(r=0.61, p<.0001)$ and vocational strain $(r=0.64, p<.0001)$. Exhaustion correlates with psychological strain $(r=0.61, p<.0001)$, role boundary $(r=0.53, p<.0001)$, and self-care $(r=-0.21, p<.0004)$. Professional efficacy correlates with rational/ cognitive coping $(r=0.36, p<.0001)$. Conclusions: A significant proportion of GCs indicate that they are experiencing occupational burnout and GC specific burnout is positively associated with occupational stress. Specific correlations identified between GC burnout and occupational stress highlight key risk factors and point to target areas for intervention and self-care. Addressing GC burnout is integral to work force strategies aimed at maintaining retention and promoting professional growth.

Behind Laboratory Doors: Lab Genetic Counselors' Experiences, Professional Identity, and Unique Ethical and Professional Challenges

C. Koellner ${ }^{1}$, P. McCarthy-Veach ${ }^{1}$, D. Groepper ${ }^{2}$, B. LeRoy ${ }^{3}$, M. Bower ${ }^{4}$

1. University of Minnesota

2. Southern Illinois University School of Medicine

3. Department of Genetics, Cell Biology and Development, University of Minnesota

4. University of Minnesota Medical Center, Fairview

About $12 \%$ of genetic counselors identify their primary work setting as a diagnostic laboratory, yet little research concerns this subspecialty. This study examined laboratory genetic counselors' experiences, and explored in greater depth ethical and professional challenges previously identified by Groepper et al. There were 5 major research questions: 1) Why do genetic counselors work in laboratory genetic counseling positions and how do they describe their position to others? 2) What is most and least rewarding about the position? 3) Do lab genetic counselors need different skills? 4) How do they believe non-lab genetic counselors, patients, and other healthcare professionals view them? 5) What ethical/professional challenges arise concerning communicating technical information to providers and conflict of interest (COI)? Fourteen lab-based genetic counselors participated in semistructured phone interviews. Most had 5-10+ years lab experience. Prevalent themes include: perceiving themselves as liaisons between the lab and ordering provider; finding helping others and intellectual stimulation as most rewarding, and administrative tasks and workload as least rewarding. Many said lab counselors do not need a unique skill set, while several said they need special technical knowledge. Many reported being perceived as valuable resources by others, but a number described occasional negative experiences (e.g., viewed as lacking people skills for a clinical position, and interfering with ordering provider wishes). Many said no COI exists when lab genetic counselors also see patients, some felt COI is possible, and some stated potential COI exists in all settings. Almost everyone reported technical communication challenges with ordering providers and offered strategies for their resolution (e.g., customized education). Results suggest laboratory genetic counselors enjoy their positions, generally are well-regarded, and encounter unique challenges due to their liaison role. Additional findings, illustrative examples, and practice and research implications are presented.

\section{Genetics Laboratory Directors' Perspectives on the Role of Genetic Counselors in Acquired Mutation Testing: Current and Expanded Opportunities}

\author{
C. Lewis ${ }^{1}$, V. Pilipenko ${ }^{2}$, S. Knapke ${ }^{2}$, T. Smolarek ${ }^{2}$, J. Everett ${ }^{3}$, K. Sund ${ }^{2}$ \\ 1. Genetic Counseling Graduate Program, University of Cincinnati \\ 2. Division of Human Genetics, Cincinnati Children's Hospital Medical Center \\ 3. University of Michigan
}

Advances in genetic testing have enabled the characterization of acquired mutations. Cancer genomic profiling can improve personalized oncology practices by helping to classify cancer types, guide treatment decisions, and provide alternative therapies. The genetic counselor's (GCs) role in 
laboratory testing for constitutional genetic mutations has been well established; however, the opportunity for GCs to apply their skills to acquired mutation testing has not been previously studied. The purpose of this study was to describe the roles for laboratory GCs in acquired mutation testing and to discuss types of training to prepare GCs for these roles. An online survey was sent to 387 clinical genetics laboratory directors. To be eligible, participants had to identify as directors of clinical laboratories offering genetic testing for acquired mutations. Thirty-nine participants met eligibility criteria and completed the survey. Twenty participants worked in a laboratory that employed a GC (Group A) and 19 participants did not employ a GC (Group B). Most of the GCs in Group A spend 1-25\% of their time on acquired mutation testing (65\%). Their primary role related to acquired mutation testing is to act as a provider resource. Other GC roles related to acquired mutation testing were reported to be similar to those performed in a constitutional mutation setting. Group A and Group B significantly differed on the level of agreement regarding the role of GCs in acquired mutation testing, with Group A agreeing more strongly for a GC role compared to Group B $(p=0.05)$. GC training programs and on the job training were cited as the top ways to prepare GCs for a role in acquired mutation testing. Laboratory GCs are currently performing roles related to acquired mutation testing. There is support for expanded GC roles in this area, particularly as perceived by directors who currently employ GCs. This work supports GC training programs incorporating coursework on laboratory-based genetic counseling including topics to prepare GCs for a role in testing for acquired mutations.

\title{
Assessing the Practices of Genetic Counselors regarding Head Circumference Measurement in Hereditary Cancer Assessment
}

\author{
A. Matchette ${ }^{1}$, E. James ${ }^{2}$, J. Shaffer ${ }^{3}$, L. Butler ${ }^{3}$, M. I. Kamboh ${ }^{3}$ \\ 1. University of Pittsburgh \\ 2. Allegheny Health Network \\ 3. Graduate School of Public Health, University of Pittsburgh
}

PTEN, a tumor suppressor gene, has been identified as an overgrowth susceptibility gene causing Cowden syndrome (CS). CS is characterized by an increased risk for breast cancer and other malignancies, mucocutaneous lesions, and macrocephaly. Macrocephaly is a hallmark of CS, is considered a major criterion for the clinical diagnosis, and is present in an estimated $80 \%$ of individuals diagnosed with CS. However, it is unknown what percentage of genetic counselors routinely measure head circumference (OFC, occipital frontal circumference) when evaluating patients for hereditary cancer assessment. This study queried National Society of Genetic Counselors (NSGC) members about current practices and opinions regarding OFC measurement. A questionnaire was dispersed electronically to all members of NSGC, and those who have practiced cancer genetic counseling in the last 6 months were eligible to respond. The data from 204 surveys was analyzed using descriptive statistics and qualitative methods including thematic analysis. $84 \%$ of genetic counselors are not measuring OFC on every patient presenting for hereditary cancer assessment, nor those who are specifically presenting for hereditary breast cancer assessment. Thematic analysis revealed these individuals feel OFC measurement should not be standard in a cancer assessment, but reserved for those who are suspicious of CS based on personal or family history. Additionally, some genetic counselors expressed they have not received appropriate training in OFC measurement. However, the $29 \%$ of genetic counselors who believe OFC should routinely be measured felt OFC is a quick and easy measurement that is helpful in assessment and is good clinical practice. Thematic analysis also revealed mixed responses about the value of OFC measurement with the increasing use of next generation sequencing panels. This supplements the literature regarding the practices and opinions of genetic counselors on OFC measurement and revealed thought-provoking differences in opinion about the value of OFC measurement in hereditary cancer assessment.

\section{A Review of the Current State of Clinical Pharmacogenomic Testing: An Examination of Existing and Potential Roles for Genetic Counselors and Pharmacists}

\section{A. McKittrick ${ }^{1}$, E. Balkite ${ }^{1}$ \\ 1. Sarah Lawrence College}

Pharmacogenomics (PGx) is a growing subset of medical genetics which examines the relationship between a patient's genotype and the ability of that patient to metabolize medications. Previous research shows that genetics can account for up to $90 \%$ of variability in drug response, and that adverse drug effects, which are the fourth most common cause of death in the United States, can be reduced by using PGx testing. Five genetic counselors, four pharmacists, and a medical doctor were interviewed in a qualitative study about their degree of involvement and experiences with PGx testing, and any barriers to widespread implementation of a PGx service that they have encountered. Primary barriers that study participants identified were: physician education/awareness, uniform insurance coverage/reimbursement, a lack of established guidelines for how to incorporate PGx results into clinical care, and the clinical utility and validity of the tests. Study participants had varying opinions concerning whether informed consent should be part of the process, and who should be responsible for administering the consent. Participants were also divided on whether PGx testing should be offered direct-toconsumer. The discordance in current processes, the substantial barriers discussed, and the health and financial benefits of PGx testing highlight a need for further research and development into delivering widespread PGx services. As experts in genetics, genetic counselors are some of the most qualified medical professionals to spearhead this effort.

\section{Role of a Genetic Counselor in the Next Generation of Oncology Clinical Trials}

\author{
A. Varma ${ }^{1}$, C. Stanislaw ${ }^{1}$, C. Bellcross ${ }^{1}$, M. Rossi ${ }^{1}$
}

\section{Emory University}

Introduction: Personalized or precision oncology relies on tumor genomic studies to tailor treatment for oncology patients. Although genetic counselors (GCs) are uniquely suited to convey genomic results to patients, there are limited studies on the effectiveness of including a genetic counselor for patient and provider support on the oncology trial team. The purpose of this qualitative study was to assess the challenges faced by oncology personnel in therapeutic clinical trials involving genomic testing, and their attitudes toward inclusion of a genetic counselor on the clinical trial team. We have also examined the preparedness and willingness of genetic counselors to take on the role of cancer genomic counseling. Methods: Semi structured interviews 
were conducted with GCs of the Georgia Cancer Genetics Network and oncologists at their institutions. The transcripts were coded and common themes were derived using thematic analysis. Results: Twenty four interviews were completed and analyzed (12 oncology personnel and 12 GCs). Major challenges expressed by the oncology personnel were lack of time, lack of training and need for more data. The ability to communicate genomic information and patient anxiety were not considered significant barriers, however, most oncology personnel were in favor of including a counselor on the team. Oncologists' lack of time and inadequate education, and insufficient genomics training for GCs were concerns mentioned by a majority of the GCs. Most GCs were willing to shift from their current position into the role of a genomic counselor with additional training. Conclusion: Both the groups agreed on major challenges except communication. A majority of the oncology personnel were willing to include a GC in a collaborative role with more data to prove utility of this addition. Further studies and recommendations are required to delineate the role of the GC in precision oncology.

\section{Genetic Testing I}

Meanings Parents Attribute to an Answer from Whole Exome Sequencing Research

B. Blosser ${ }^{1}$, M. Myers ${ }^{1,}$, , M. Barnes ${ }^{3}$, I. Bradley ${ }^{1}$, J. Lynch ${ }^{1}$, R. Pickler ${ }^{4}$, C. Prows ${ }^{5}$

1. University of Cincinnati

2. Division of Human Genetics, Cincinatti Children's Hospital Medical Center

3. AssureRx Health, Inc.

4. Cincinnati Children's Hospital

5. Cincinnati Children's Hospital Medical Center

Introduction: Whole exome sequencing (WES) is a recent strategy for discovering causal variants of diseases, especially rare monogenic disease. The purposes of this qualitative study are to explore the reasons parents decide to have exome sequencing for their child with a rare undiagnosed disorder, to examine parents' expectations regarding WES, and to assess the meaning they attribute to anticipated results.. Methods: The target population consisted of mothers and fathers of children with rare disorders of unknown cause. Twenty mothers and 18 fathers were separately interviewed in-person or by phone within 2 weeks after informed consent. A topical guide with probes was developed and used for interviews. Interviews were audiotaped and transcribed. Transcripts were entered into NVivo, a qualitative data management software program. Two coders independently coded each transcript using interpretive phenomenological analysis. Discrepancies were resolved through discussion and themes were validated by a research committee. Results: Data were managed by first coding 3 preconceived categories: reasons, expectations, and assigned meanings. Within the 3 respective categories the following themes emerged: to find a diagnosis for their child; to help others and to do everything possible to help their child; and hope for an answer but reluctant to expect a primary result from WES. The overwhelming meaning attributed to a positive result was options become available for their child. A diagnosis would also provide closure, a roadmap of the future and an answer to tell others. Conclusion: As WES becomes more widely used in clinical settings, genetic counselors should develop strategies to assure families seeking to find a diagnosis consider the limitations of WES. Having more realistic expectations about the potential of WES may encourage parents to participate in WES studies while at the same time, minimize potential disappointment with WES outcomes.

Disomic Placentas, Trisomic Babies: Reverse Mosaicism and Implications for Noninvasive Prenatal Testing

T. Boomer ${ }^{1}$, N. Dharajiya ${ }^{1}$, W. Paxton ${ }^{1}$, A. Bombard ${ }^{1}$, C. Noonan ${ }^{2}$, N. Hoppman ${ }^{3}$, E. Highsmith ${ }^{3}$, M. Wick ${ }^{3}$, J. S. Saldivar ${ }^{1}$

1. Sequenom Laboratories

2. Maternal Fetal Medicine of Ohio Health

3. Mayo Medical Laboratories

Background: Noninvasive prenatal testing (NIPT) screening relies on the presence of circulating cell-free DNA from the pregnancy, believed to be largely placental in origin. In most pregnancies, the genetic material in the fetal tissues and the placental tissues are a match. However, a mismatch between fetus and placenta can occur, leading to discordant NIPT results. False positive NIPT results attributed to mosaicism have been previously reported. However, the first two cases of atypical "reversed" placental mosaicism resulting in false negative NIPT results are presented here. Cases: Two cases are highlighted in which NIPT results performed at 19.5 and 11.1 weeks gestation, were reported as negative. Multiple anomalies were seen on ultrasound for the first case and invasive testing was declined. Advanced maternal age was the only indication for the second case. Blood chromosome studies at birth were positive (non-mosaic) for trisomy 13 and trisomy 21, respectively, raising concern about false negative results from NIPT. Follow-up studies were performed by FISH analysis on FFPE placental tissue, demonstrating chromosomal mosaicism in the villi tissue. A range of 75-95 \% euploidy was seen across various villi sections. Conclusions: These cases describe two pregnancies affected with chromosome abnormalities at birth despite negative NIPT results. However, postpartum analysis of the placenta demonstrated that low level placental mosaicism can go undetected; representing, in essence, an insufficient fraction of aneuploid placental DNA. These cases highlight that mosaicism due to post zygotic non-disjunction or trisomy rescue, and uneven distribution and/or selection of cells between fetus and placenta is a mechanism that may lead to false negative NIPT results. Confounding factors may include normal biochemical screening results and enhanced fetal survival given the predominance of a euploid placenta. The implications for prenatal counseling are complex.

Multigene Panel Testing vs.Whole Exome Sequencing: What Is the Best Testing Approach for Patients with Epilepsy and Neurodevelopmental Disorders?

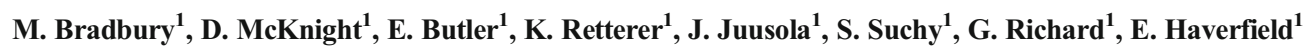

1. GeneDx 
OBJECTIVE: Genetic diagnosis for epilepsy-related neurodevelopmental disorders can be challenging due to clinical and genetic heterogeneity and abundance of genetic testing options. We have compared the positive diagnostic rate of targeted epilepsy panels to whole exome sequencing (WES) for these patients. METHODS: This was a retrospective cross-sectional study of $\sim 3,500$ patients with epilepsy who were referred for diagnostic testing using various multi-gene epilepsy panels (next generation sequencing and deletion/duplication testing) compared to a cohort of 171 patients with neurodevelopmental disorders (NDD) and epilepsy referred for diagnostic testing using WES. RESULTS: Epilepsy panel testing revealed a clinical sensitivity of $15 \%(522 / 3,490)$ with a higher diagnostic rate for a subset of genes linked to early-onset epilepsy $(20 \%$; 218/1,132). In contrast, patients with NDD and epilepsy had a $34 \%$ positive rate by WES (58/171); however, only $10 \%$ had mutations in the same set of genes sequenced on the panels (17/171). This discrepancy may be a combination of incomplete coverage and missed exon-level deletions/duplications by WES and sampling bias due to exclusion of patients with prior positive findings. The majority of WES positive results were identified in genes not on the epilepsy panels. WES-trio analysis was more successful in making a molecular diagnosis (36 \% of families; 54/148) than proband-only analysis (22 \% of patients; 5/23). When using a tiered approach, $27 \%(8 / 30)$ of individuals with negative panel testing had a positive result on subsequent WES analysis, demonstrating the benefits of a combined approach. CONCLUSIONS: This data suggests that patients with NDD and epilepsy may benefit from a tiered testing approach including both targeted gene panels and WES, which could yield a diagnosis in about $40 \%$ of patients. This may have implications for pre-test informed consent discussion, which should address the range of possible findings in both tests, as well as the limitations and benefits of this tiered approach to testing.

Genomes, Exomes and Targeted Disease Panels: Understanding their Relative Strengths and Weakness When Providing Genetic Counseling

\section{Campbell ${ }^{1}$, D. Kolbe1 ${ }^{2}$, R. Smith ${ }^{2}$}

1. Iowa Institute of Human Genetics, University of Iowa

2. Iowa Institute of Human Genetics

Whole genome (WGS), whole exome sequencing (WES), and targeted disease panels are useful tools for evaluation and diagnosis of patients. Each of these tests has strengths and weaknesses. Factors to consider when selecting a test include capture and coverage statistics, and detection of incidental and secondary findings. Understanding elements of test reports such as depth of coverage, pathogenicity scores, and classification of variants as pathogenic, benign, or unknown significance can aid genetic counselors in interpreting results. Two factors impacting test selection are capture and sequencing coverage performance. Since, none of the whole exome platforms capture the entire exome, knowing if candidate genes will be captured and their typical sequence coverage is important when selecting a test. Recently, we identified a causative mutation in a child with a congenital disorder of glycosylation and negative WES result using a targeted disease panel as the causative variant was located in a gene region not captured by WES. Sequence coverage is also important when counseling patients regarding results. The American College of Medical Genetics and Genomics recommends laboratories report findings in 56 genes for clinical WES or WGS. Analysis of exome capture platforms revealed $1.0 \%$ of these genes are not captured, $10 \%$ are insufficiently sequenced and $>1 \%$ of $B R C A 2$ disease mutations in $\mathrm{HGMD}^{\circledR}$ are in regions with insufficient coverage. The ability to accurately interpret this information is critical and has implications when counseling patients, as a "negative" result may not be truly negative. Test selection requires genetic counselors to know the differences between WGS, WES, and targeted disease panels. Understanding the differences and knowing questions for laboratories empowers genetic counselors to select the best test for their patients. Accurate interpretation of test reports including gene regions not captured and inadequately sequenced allows genetic counselors to provide informative answers to patients.

\section{A Negative Result on Exome Sequencing: What a Genetic Counselor Should Know}

\section{G. Chandratillake ${ }^{1}$, S. Garcia ${ }^{1}$, J. Tirch ${ }^{1}$, D. Church ${ }^{1}$, M. Pratt ${ }^{1}$, M. Clark ${ }^{1}$, J. Harris ${ }^{1}$, S. Chervitz ${ }^{1}$, J. West ${ }^{1}$, R. Chen ${ }^{1}$}

\section{Personalis, Inc.}

Exome sequencing is increasingly utilized in the diagnosis of disorders of unknown genetic etiology. Despite increasing evidence for the utility of this approach, the majority of patients presenting with a presumed Mendelian genetic trait receive a negative sequencing report. Both technical and biological factors contribute to the current difficulty in deriving diagnoses from genomic sequence information, and genetic counselors ordering exome sequencing tests should be aware of the following issues. Whole exome sequencing is not as comprehensive a test as it sounds. Many genes with clinical relevance are not well-covered by standard exome sequencing, and average coverage statistics do not necessary reflect good gene coverage either. Disease-causing variants can simply be missed if the coverage for a particular gene is not sufficient across the whole gene consistently in every sample. The recognition of variants in a sample relies on sequence reads being aligned to the reference genome sequence. However, there are regions of the genome, including whole genes, that are missing from the current reference assembly. These "missing sequences" in the reference cause reads to be misaligned resulting in both false positive and false negative variant calls. Certain types of variants e.g. large insertions or deletions, are not routinely recognized by exome sequencing, nor are variants that fall outside the coding regions of genes. These represent another source of "missed diagnoses". Additionally, variant classification relies on a robust understanding both of the genetic variation that exists in the population as a whole as well as the phenotypic spectra of diseases, both of which are rapidly evolving. It is important that a genetic counselor engaged in ordering exome sequencing for their patients understands the various issues that can hamper a diagnosis being made by this approach. Familiarity with such issues enables informed test selection decisions, proactive discussions with laboratories, and appropriate follow-on patient care.

Exploring Parental Perspectives on the Return of Genomic Results for Children Enrolled in a Pediatric Genetic Biorepository

P. Connors ${ }^{1}$, S. Savage ${ }^{2}$, S. Ziniel ${ }^{2}$, C. Wang ${ }^{3}$, K. Dies ${ }^{2}$, M. Campion ${ }^{1}$, I. Holm²

1. Boston University School of Medicine

2. Boston Children's Hospital

3. Boston University School of Public Health 
The genetics community is in the midst of delineating best practices for analyzing and returning genomic results in both the clinical and research realms. While professionals have yet to reach consensus on this complicated and challenging subject, it is unclear where patients and their families stand on these issues. To address this paucity of data, a survey was distributed to a group of parents of children enrolled in a pediatric genomic biorepository with anticipated return of research-derived results. The survey investigated parents' desire to receive their children's genomic results and their anticipated responses to such information. Seven hypothetical result return scenarios were presented so that respondents' opinions and expected reactions could be compared across various disease categories. Implications about placing results in the medical record and points of view regarding child's future autonomy were also explored. Results obtained from 178 complete responses ( $21.2 \%$ response rate) indicated that an overwhelming majority of parents wish to learn about results related to conditions across a spectrum of perceived disease severity, preventability, and age-of-onset. The percentage of respondents expressing desire to know about any of these conditions ranges from a minimum for Alzheimer's disease to a maximum for familial adenomatous polyposis $(84.3 \%$ and $99.4 \%$, respectively). Participants also report a strong likelihood of seeking genetic testing for themselves and their other children for many of these conditions, as well as seeking care from pediatricians, primary care providers, specialists, geneticists, and genetic counselors related to these research-derived findings. Furthermore, a majority of participants support placing these potential results in their child's medical records across most scenarios, though the degree of support varies by condition. This study demonstrates a strong desire for a wide variety of genetic information, and sheds light on the potential impact these research endeavors might have on the medical community at large.

\title{
The Importance of Clinical Indications in the Analysis and Interpretation of Next Generation Sequencing Gene Panel Data
}

\author{
G. Douglas ${ }^{1}$, V. Zhang ${ }^{1}$, J. Wang ${ }^{1}$, F. Li ${ }^{1}$, Y. Wang ${ }^{1}$, S. Peacock ${ }^{1}$, E. Schmitt ${ }^{1}$, B. Graham ${ }^{1}$, C. William ${ }^{1}$, L. J. Wong ${ }^{1}$
}

\section{Baylor College of Medicine}

Clinical correlation in individuals submitted for genetic testing has always been an important part of the accurate and timely interpretation of diagnostic results. As technical advances in sequencing have allowed greater use of multiple gene panels, and as their popularity grows, the precise clinical indication for testing plays an even greater role. Providing a clear indication to the diagnostic laboratory limits the amount of time spent on internal discussions, consultations, and follow up communications to obtain indications. Even a few key clinical features can greatly aid the diagnostic testing process and interpretation of results. In our experience in developing over 40 different multi-gene panels at the Medical Genetics Laboratories (MGL) at the Baylor College of Medicine, having clinical indications appropriate for a given panel correlates with higher detection rates, and has aided us in solving a variety of complicated cases, including identifying deletions based on NGS copy number analysis, uncovering allele drop out, detecting unusual mutations, and identifying low level mosaicism. Although such cases represent a relatively small portion of the overall number of cases we test and solve, each diagnosis is personally important to the individual or families involved. Knowing the specific symptoms and degree of clinical suspicion assures that due diligence is given to candidate loci. Genotype-phenotype correlation data maintained by the laboratory is also important in the interpretation of findings in subsequent cases. Given that genetic counselors play a key role in both the ordering of genetic testing and relaying the results back to patients, they play a pivotal role in this flow of information. Supporting the need to include clinical indications for genetic testing by everyone, including counselors, physicians, and laboratory personnel, helps maintain high standards of testing for our patients and helps grow and advance our shared field of genetics.

Identification of Recurrent De Novo Alterations in the Clinically Novel PURA Gene through Diagnostic Exome Sequencing

D. El-Khechen ${ }^{1}$, L. Shahmirzadi ${ }^{1}$, C. Mroske ${ }^{1}$, T. Grebe ${ }^{2}$, B. Kraeer ${ }^{2}$, N. Shur ${ }^{3}$, K. Miller ${ }^{3}$, S. Naidu ${ }^{4}$, J. Cohen $^{4}$, W. Zeng ${ }^{1}$, S. Tang ${ }^{1}$

\author{
1. Ambry Genetics \\ 2. Phoenix Children's Hospital \\ 3. Albany Medical Center \\ 4. Kennedy Krieger Institute
}

Diagnostic exome sequencing (DES) succeeds in finding a diagnosis for 30-40 \% of undiagnosed patients with underlying Mendelian disorders, with approximately $15 \%$ of these being novel gene findings. Here we describe three patients with alterations in the novel gene PURA identified through DES and delineate the phenotype. Located on chromosome 5q31, the Purine-Rich Element-Binding Protein A (PURA) gene encodes a 322 amino acid protein that is highly conserved in eukaryotes. Pur-alpha is a sequence-specific single-stranded DNA and RNA-binding protein that functions as a transcriptional activator. PURA plays an important role in postnatal brain development by regulating the expression of neuronal RNA in dendrites. PURA haploinsufficiency is postulated to account for the neurological symptoms seen in 5q31.3 microdeletion syndrome. Patient 1 is a 3 -year old Caucasian boy with congenital hypoglycemia, pronounced hypotonia, developmental delay, recurrent infections and poor weight gain. A muscle biopsy was consistent with mitochondrial dysfunction. DES identified a de novo frameshift alteration in PURA (c.927_940del/ p.T310Gfs*2). Patient 2 is a 5-year old Caucasian boy with developmental delay, dysmorphic features, severe congenital hypotonia, neonatal seizures, and history of failure-to-thrive (FTT). A de novo 7q21.3q21.2 microdeletion was deemed to be not significant. DES identified a de novo frameshift alteration in PURA (c.881dupA/p.L295Afs*22). Patient 3 is a 6-year old Caucasian boy with developmental delay, congenital hypotonia, cortical atrophy, and history of FTT. DES identified a de novo missense alteration in PURA occurring at a highly conserved amino acid position located in the DNA/RNA binding domain (c.305 T $>$ G/L102R). These data support the role of PURA alterations as a cause of a new neurodevelopmental syndrome characterized by the major features of developmental delay, hypotonia and poor growth. Our results also highlight the utility of DES in the identification of novel genes and have significant implications for genetic counseling and clinical management.

\section{Homocystinuria Diagnosed by Whole Exome Sequencing in Siblings from an Isolated Central American Village}

C. Farkas ${ }^{1}$, J. A. Lefferts ${ }^{2}$, M. A. Cervinski ${ }^{2}$, M. B. P. Dinulos ${ }^{2}$, T. Gallagher ${ }^{2}$, H. Steinmetz ${ }^{2}$, S. Turner ${ }^{2}$, S. Vallee ${ }^{2}$, R. Chen ${ }^{1}$, S. Garcia ${ }^{1}$, D. Church ${ }^{1}$, J. Tirch ${ }^{1}$, G. Tsongalis ${ }^{2}$, A. Huang ${ }^{1}$ 
1. Personalis, Inc.

2. Dartmouth-Hitchcock Medical Center

Introduction: Homocystinuria is an autosomal recessive metabolic disorder in which elevated concentrations of methionine and homocysteine result from a defect in the conversion of homocysteine to cysteine. The disorder is caused by mutations in the cystathionine beta-synthase gene (CBS) and presents with a variable phenotype. Features generally include developmental delay, intellectual disability, skeletal abnormalities, ocular defects, osteoporosis, and thromboembolism. Case Report: We present the case of a family from an isolated Central American village with two children similarly affected by developmental delays, intellectual disability and dysmorphic features of unknown etiology. DNA samples were extracted from buccal swabs from two affected siblings and their mother. Augmented whole exome sequencing (WES) was performed on DNA from the oldest sibling (ACE clinical exome, Personalis). A high-level of homozygosity was detected in the proband, with $14.4 \%$ of the exome contained within stretches of homozygosity $>4 \mathrm{Mbp}$. A homozygous novel frameshift variant in $C B S$ was also detected. This deletion of a single nucleotide is considered likely pathogenic. A diagnosis of homocystinuria was confirmed in this patient by detection of an elevated blood homocysteine concentration. The presence of the variant was confirmed by Sanger sequencing in the other affected sibling (homozygous) and in the unaffected mother (heterozygous). Discussion: This report of undiagnosed homocystinuria highlights several relevant reminders for genetic counselors as we move forward in this era of global medicine and genomic testing. Genetic diseases that are part of many newborn screening efforts should not be overlooked when counseling patients without access to standard U.S. medical services. WES is an effective testing strategy for facilitating diagnosis when an unknown genetic disorder is suspected.

\title{
Use of an Enhanced Exome with Genome-Wide Structural Variant Detection for the Diagnosis of Mendelian Disease
}

\author{
S. Garcia ${ }^{1}$, J. Tirch ${ }^{1}$, J. Harris ${ }^{1}$, A. Patwardhan ${ }^{1}$, D. Church ${ }^{1}$, M. Clark ${ }^{1}$, G. Chandratillake ${ }^{1}$, M. Pratt ${ }^{1}$, J. West ${ }^{1}$, R. Chen ${ }^{1}$ \\ 1. Personalis, Inc.
}

Introduction: Despite the theoretical power of exome sequencing, the realized diagnostic yield of clinical exome testing remains relatively low, $\sim 25 \%$. False negatives, which may account for a significant proportion of unsolved cases, stem from both technical and analytical challenges, including inadequate sequencing coverage, undetected variation (e.g. structural variation), non-optimized prioritization of variants, and limited understanding of the impact of genetic variation on phenotype. Hypothesis: The development of approaches to address these challenges, including the development of additional sequencing-based assays to address gaps in sequencing coverage and to detect structural variation, extensive annotation to aid in the interpretation of variants, and a phenotype-based analysis approach to decrease turn-around-time will increase diagnostic yield. Methods: We used the ACE (Accuracy and Content Enhanced) Clinical Exome to analyze $>50$ cases of undiagnosed disease; $>50$ additional cases are currently in review. Results: Variants in genes associated with phenotype were reported in $56 \%$ of cases, and included several structural variants. The majority of the genes in which we reported variation showed deficits in sequencing coverage on standard exome platforms. In a male with retinitis pigmentosa, a likely pathogenic variant in $R P G R$ was identified in an exon with extremely poor coverage on standard exomes. Conclusions: We demonstrate the ACE Clinical Exome's diagnostic yield in cases of undiagnosed disease. The ACE Clinical Exome improved sequence coverage over genes that harbored likely disease-associated variants and was able to detect structural variation. Nearly half of the samples analyzed to date had had clinical aCGH testing performed prior to exome analysis, and thus are likely depleted for disorders explained by large structural variation. Diagnostic yield of an integrated exome/CNV platform is likely to be further increased above that observed if used as a first line diagnostic for patients with complex phenotypes.

Prenatal Screening and Testing in the Noninvasive Era: A New Paradigm in Patient Desire?

\author{
A. Hanson ${ }^{1}$, S. Hashmi ${ }^{2}$, B. Stevens ${ }^{3}$, P. Robbins Furman ${ }^{4}$, J. Refuerzo ${ }^{5}$, C. Singletary ${ }^{6}$ \\ 1. University of Texas Graduate School of Biomedical Sciences \\ 2. Department of Pediatrics, University of Texas Medical School \\ 3. University of Texas \\ 4. Baylor College of Medicine \\ 5. University of Texas Health Science Center at Houston Medical School \\ 6. University of Texas Health Science Center at Houston
}

Noninvasive prenatal testing (NIPT) is a highly sensitive and specific screen for common aneuploidies that eliminates the risk of miscarriage carried by diagnostic tests. This study aimed to explore how this new prenatal option impacts pregnant women's decision-making process. Women undergoing genetic counseling from September 2013 to February 2014 at a University of Texas Medical School Maternal Fetal Medicine Clinic who were 18 or more years of age, English or Spanish speaking, and who would be offered NIPT during their genetic counseling session were eligible to participate in an anonymous pre-counseling survey. Information about test uptake was completed by the genetic counselor post-counseling. Multivariatble statistical analysis and backwards stepwise logistic regression were performed on 149 collected surveys to determine what factors influence NIPT uptake, and thematic analysis was conducted. Analysis of 17 factors indicated that the factors that positively influence the uptake of NIPT are: desire to know if a pregnancy is affected ( $p=0.005)$, willingness to consider NIPT ( $p=0.011)$, some college education $(p=0.009)$, and primary Spanish-speaking $(p=0.013)$. Overall uptake of NIPT was $73.82 \%(n=110)$ and of amniocentesis was $6.04 \%(n=9)$. A small but important group of women was identified (17.5 \%) who declined all testing and screening options despite the option of NIPT. Interestingly, separate analysis of 16 women who had NIPT prior to their genetic counseling appointment showed only $37.5 \%$ properly identified already having NIPT. The presence of a group with an apparent lack of informed consent and a distinct group of women who decline all prenatal screening supports the need for genetic counseling to promote informed decision making when women are offered prenatal screening and testing options.

\section{Preferences of Undergraduate Students towards the Reporting of Incidental Findings in Whole Genome and Whole Exome Sequencing}

\section{Hong ${ }^{1}$, B. O'Connor ${ }^{1}$, K. Owens ${ }^{1}$, B. Yashar $^{1}$, J. Schuette ${ }^{2}$}


1. University of Michigan

2. University of Michigan Health System

The 2013 American College of Medical Genetics and Genomics (ACMG) recommendations for reporting incidental findings (IFs) in whole genome/ exome sequencing (WGS/WES) have generated controversy within the genetics community. Previous studies regarding preferences for return of results have queried genetics professionals and research participants. We chose to explore the preferences of undergraduates, as future consumers of WGS/WES. A novel 34-item survey was developed to explore opinions about patient autonomy/choice, results disclosure, and genetic testing in minors. Likert scale, multiple choice, and demographic items were included after consultation with experts in survey design to ensure readability and validity. The web-based survey was administered to 5,003 University of Michigan undergraduates in March 2014. A total of 381 responses (8\%) were included in statistical analyses (descriptive and comparative). Participants were mostly healthy Caucasians majoring in science without personal or family experience with genetic counseling. Although participants were divided about who should decide to order IFs (patient vs personal physician vs genetics professional), the majority $(62 \%)$ indicated the patient was the best person to decide whether these results should be reported; being female was a significant predictor of this selection ( $p=0.001$ ). When given the hypothetical option for IF analysis, $10 \%$ of respondents would not elect this testing, and of these, $70 \%$ agreed that the laboratory should not perform IF analysis if choosing to opt out. The choice to select among the ACMG recommended conditions and the ability to change decisions about results disclosure were desired by the majority ( $70 \%$ and $77 \%$, respectively). Responses were divided on the issue of genetic testing in minors; up to $68 \%$ favored disclosing incidental results to benefit a parent. Of those preferring to protect minor choice, more were females (37 vs $22 \%, p=0.003$ ). These results provide novel perspectives on individual preferences for IFs. Such information should be incorporated into discussions surrounding the return of results from WGS/WES analysis.

\title{
Trends in a Rapidly Expanding Genetic Testing Marketplace
}

\section{G. Hooker ${ }^{1}$, T. A. Murphy ${ }^{1}$, C. Winfree ${ }^{1}$, J. Staples ${ }^{1}$, J. Marshall Cottrell ${ }^{1}$, B. Blackshear ${ }^{1}$, J. Schneider ${ }^{1}$}

\section{1. $N e x t G x D x$}

The landscape of available genetic tests is rapidly changing, presenting increasing opportunities more effective and more efficient application of genetic data to patient care. Anecdotal evidence would indicate that the number of clinically available tests is larger than has been previously estimated and that the marketplace is changing at a rapid pace. The goal of this analysis was to estimate the number of tests available in the genetic testing marketplace and explore specific areas of expansion in clinically available genetic tests. The NextGxDx bioinformatics team has developed and implemented technology to extract information on available genetic testing products from 205 CLIA-certified US laboratories (to date), standardize requisite terminology, and curate these products into comparable and clinically relevant categories within our publicly available database. We performed a descriptive analysis of the genetic tests in the NextGxDx database and evaluated trends observed from January 1, 2014 to May 7, 2014. As of May 7, 2014, our database contained 19,329 clinically available genetic tests. Of these 3,737 of the tests were gene panels of two or more genes, and 1,024 had 5 or more genes. Among the laboratories included in our database at the start of 2014, 886 new tests have been launched. Of the 5 laboratories with the most rapidly growing test catalogs, an average of 59 new tests are coming onto the market each month. Of the tests added by these labs in the month of April, nearly half of the tests $(45 \%)$ were panel tests of 5 or more genes. Given these results, we anticipate that there will soon be over 20,000 clinically available genetic tests, a growing number of which are complex multi-gene panels. Recognizing the changing landscape of these tests, continuation of ongoing educational efforts and resource development to assist genetic counselors in adapting to these changes is warranted.

\section{Whole Genome Sequencing of De Novo Balanced Chromosome Rearrangements Empowers Prenatal Genetic Counseling}

\author{
T. Kammin ${ }^{1}$, Z. Ordulu ${ }^{1,2}$, M. E. Talkowski ${ }^{2,3,4,5}$, J. F. Gusella 2, 3, 4, 5, 6, C. C. Morton ${ }^{1, ~ 2, ~ 5, ~} 7$ \\ 1. Department of Obstetrics, Gynecology and Reproductive Biology, Brigham and Women's Hospital \\ 2. Harvard Medical School \\ 3. Center for Human Genetic Research, Massachusetts General Hospital \\ 4. Department of Neurology, Massachusetts General Hospital \\ 5. Program in Medical and Population Genetics, Broad Institute of Harvard and MIT \\ 6. Department of Genetics, Harvard Medical School \\ 7. Department of Pathology, Brigham and Women's Hospital
}

Introduction: Every year $\sim 150$ babies are born with de novo balanced chromosome rearrangements (BCR) in the USA. Up to $20 \%$ of these will have an abnormal outcome likely due to disruptions or dysregulations of genes in breakpoint regions. Nucleotide level precision for gene identification is not routinely available in cytogenetic laboratories, making it difficult to counsel patients faced with prenatal clinical decisions. The Developmental Genome Anatomy Project uses whole genome sequencing (WGS) to explore BCR. This case study illustrates how this approach helps address counseling dilemmas raised by prenatal detection of a de novo BCR. Case Report: Our patient had an amniocentesis at 16 weeks for advanced maternal age. The GTG-banded karyotype revealed a de novo paracentric inversion: 46,XY,inv(8) (q13q24.1)dn. Our research study used a large insert "jumping library" WGS approach to explore the rearrangement, revising it to: 46,XY,inv(8) (q11.21q24.23)dn. The inversion disrupted KHDRBS3, a putative RNAbinding protein involved in alternative splicing. Based on convergent genomic data from multiple sources our consensus interpretation was this inversion was of unknown clinical significance, with no available data to suggest it was pathogenic. After CLIA validation, the parents chose to continue the pregnancy and at 18 months the child is healthy showing normal development. Discussion: The news that your unborn child carries a de novo BCR is shocking and unexpected. We received feedback from the mother with her thoughts on the case and explored her feelings using counseling theory. Parents facing this situation are often counseled based on the GTG-banded karyotype and CGH array. In such situations, conventional cytogenetic methods are not sufficiently precise to provide optimal information for parents to make the most informed decision. We find WGS approaches are a viable, accurate and timely method of refining chromosome rearrangement breakpoints in prenatal cases and warranted for implementation in clinic, empowering counselors and patients faced with de novo BCR. 


\title{
Assessing Factors Affecting the Decision regarding Noninvasive Prenatal Testing after Genetic Counseling
}

\author{
K. Kobara ${ }^{1}$, L. Hudgins ${ }^{2}$, Y. Blumenfeld ${ }^{3}$ J. Taylor $^{4}$
}

1. Department of Genetics, Stanford University

2. Department of Pediatrics, Division of Medical Genetics, Stanford University. Lucile Packard Children's Hospital at Stanford

3. Department of Obstetrics and Gynecology, Division of Maternal Fetal Medicine, Stanford University

4. Lucile Packard Children's Hospital Stanford, Stanford University

Question: The objective of this study is to assess uptake of noninvasive prenatal testing (NIPT) and various factors that may be influencing women's decision to accept or decline NIPT after genetic counseling. Purpose: The information from this study may help genetic counselors to better understand what factors are driving women's decisions regarding NIPT and provide further insight into how NIPT is fitting into the previously well-established prenatal testing paradigm. Methods: Women were recruited from the Perinatal Diagnostic Center at Lucile Packard Children's Hospital Stanford and completed a written questionnaire after their genetic counseling appointment. Women were also asked to participate in an optional online follow-up survey assessing insurance coverage of NIPT. Results: Ninety-eight women completed the written questionnaire. Of the total respondents, 42 (42.9 \%) elected and 56 (57.1\%) declined NIPT after genetic counseling. Most women (80.9\%) electing NIPT chose to have routine aneuploidy screening as well. Factors that were found to be associated with electing NIPT were older age, higher education, prenatal screening in a prior pregnancy, history of infertility and awareness of the test prior to genetic counseling. Respondents electing NIPT most commonly reported safety (35.7 \%) and accuracy (35.7\%) as the most important factors influencing their decision. A total of 17 respondents completed the follow-up questionnaire assessing insurance coverage of NIPT. Most of these respondents ( $71 \%$ ) who elected NIPT reported at least some coverage of NIPT by their insurance companies. Conclusion: The paradigm of prenatal screening and testing is continuing to evolve with increasing uptake of NIPT over time. Most women who elected NIPT are choosing to have the test in addition to, rather than instead of, routine screening. This shift towards increased utilization of NIPT can be attributed to a variety of factors including increased awareness of the test by patients and providers and adequate insurance coverage.

\section{Pharmacogenomics: An Analysis of the Baseline Knowledge of Clinseq ${ }^{\circledR}$ Participants}

\section{J. Kohler ${ }^{1}$, K. Lewis ${ }^{2}$, L. Biesecker ${ }^{3}$, B. Biesecker ${ }^{4}$}

1. Johns Hopkins University/National Human Genome Research Institute

2. National Institues of Health

3. National Human Genome Research Institute, Genetic Disease Research Branch

4. Genetic Counseling Training Program, Johns Hopkins University/National Human Genome Research Institute

Pharmacogenomic (PGx) testing is poised to become one of the first applications of genome sequencing to achieve personalized medicine. Expected outcomes include enhanced drug efficacy and reduction in adverse drug responses. Several studies have reported general public support for PGx testing but overall lack of knowledge. The objective of this study was to evaluate PGx knowledge as compared to other genome knowledge among participants in the ClinSeq ${ }^{\circledR}$ cohort. We hypothesized that as related but distinct concepts they would be positively correlated. ClinSeq ${ }^{\circledR}$ aims to assess the overall clinical utility of whole-genome sequencing. The study population was selected to represent the spectrum of atherosclerotic heart disease using the Framingham score to balance accrual. 546 enrolled participants completed a baseline survey assessing their knowledge of various aspects of genome sequencing, measured by 16 Likert-type items. One item pertained to PGx: "genome sequencing may find variants in people's genes that may determine how they respond to certain medicines" $(1=$ definitely no, $5=$ definitely yes $)$. For this analysis, we stratified data into two groups by response to the PGx item (correct $=4-5, n=376$; incorrect $=1-3, n=162$ ) and compared group means for the remaining 15 items using a chi-squared test. 12 of 15 items showed significantly different means $(p<.03)$ while 3 did not $(p>.05)$, indicating PGx knowledge is positively correlated with most other key concepts representing knowledge of genome sequencing. People who have greater baseline knowledge of genomics tend to have a greater knowledge of PGx. Results suggest that PGx is recognized as a key application of genome science by informed participants that we anticipate are eager to learn PGx results. While PGx tests are considered to have low psychosocial risk, our findings affirm the need for appropriate pre- and post-test education and counseling as practiced with other genetic tests.

\section{Assessing Patient Comprehension Using a Modified Consent Document in a Pharmacogenomics Study}

\author{
S. Lahiri' , S. Aufox ${ }^{2,3}$, V. Pan $^{4}$, M. Smith ${ }^{2,3}$ \\ 1. Graduate Program in Genetic Counseling, Northwestern University \\ 2. Center for Genetic Medicine, Northwestern University, \\ 3. NUgene Project \\ 4. Advocate Lutheren General Hospital
}

Genetic counselors are recruiting for an increasing number of genomic studies. However, there is concern over the increasing length and complexity of consent forms used in these studies, which has been associated with a decline in participants' comprehension of study information. Research genetic counselors struggle with using forms that have high reading levels due to the complicated nature of genetic information. Shorter consent forms containing minimal study information, that meet all the ethical, federal, and IRB requirements, along with an informational brochure have been proposed as a solution. We evaluated the impact of a shortened consent form on comprehension for a pharmacogenomics study. Over 3 months, 62 participants were recruited and consented for a pharmacogenomics study using a consent script in an internal medicine clinic. Thirty-two participants were consented using the standard consent form ( 6 pages) and 28 were consented with a shortened form (4 pages). Both forms were accompanied by a brochure containing detailed study information. All participants were surveyed about study comprehension and perceptions of the consent form and brochure. Responses were compared between the two consent groups and a sub group of participants who chose not to read the consent form. Out of the 62 participants, 32 reported reading the form. The mean objective comprehension scores for those consented with the standard consent form, the shortened 
form, and those who did not read were $8.2(\mathrm{SD}=0.8), 8.1(\mathrm{SD}=1.0)$, and $8.3(\mathrm{SD}=0.8)$ respectively. No participants found the consent forms to be lacking necessary information. We conclude that, although the consent form is only a part of the informed consent process, using a shortened consent form did not negatively impact comprehension. Furthermore, participants felt the shortened consent addressed all of the important information necessary to decide about study participation. Genetic counselors should consider using a shortened consent for genomic studies.

\title{
Medical EmExome: Making "Whole" Exome Sequencing Truly Whole!
}

\author{
A. Narravula ${ }^{1}$ L. Catalano ${ }^{2}$, C. da Silva ${ }^{1}$, M. Hegde ${ }^{1}$ \\ 1. Emory Genetics Laboratory \\ 2. Emory University
}

With the advent of whole exome sequencing (WES), many laboratories adopted commercially available kits such as Nimblegen's SeqCap EZ Human Exome Library v3.0 and Agilent's SureSelect Human All Exon V5 Clinical Research Exome. These kits target only 85-90 \% of the exome and can fail to identify pathogenic variants in genes associated with disease (GADs). For this study, 2,593 variants classified as pathogenic by Emory Genetics Laboratory (EGL) in 258 GADs were identified. The coverage data for these 2,593 variants for 6 samples sequenced by Nimblegen's v3.0 kit showed that $374(14.4 \%)$ of 2,593 variants had no or low coverage $(<20 \mathrm{X})$ in $>=1$ sample. To address this gap, EGL created the Medical EmExome to the enhance coverage of exons of 4,600 GADs in OMIM. Using Agilent's V5 kit as the backbone, EGL added additional probes for the low and no coverage exons of these genes to enhance coverage to approximately $98-99 \%$. When the coverage data for the same 2,593 pathogenic variants was analyzed from 5 samples sequenced by the Medical EmExome, only $66(2.5 \%)$ of 2,593 variants had low or no coverage in $>=1$ sample. This study highlights the fact that WES is incomplete and that ordering providers should be conscious that known pathogenic variants, which could be identified on a NGS panel or by Sanger sequencing, might be missed. Also, an understanding of the difference in coverage between the different WES tests available might help one choose a test with the most clinical utility and sensitivity. Clinicians should also consider if a laboratory provides coverage information for genes-ofinterest and if regions of no or low coverage will be Sanger sequenced if a list of genes-of-interest is provided. A major advantage of the Medical EmExome is that Sanger sequencing to fill in low and no coverage exons is no longer a burden for the genes-of-interest. This allows clinicians to target phenotype-related genes at $100 \%$ coverage. This can be useful to clinicians and researchers as they can be more confident that a negative result is a true negative for known GADs and can concentrate their efforts on novel genes.

Germline STAT2 Variant Implicated through Clinical Whole Exome Testing in Patient with Familial Non-Medullary Thyroid Cancer

J. Pickard Brzosowicz ${ }^{1}$, A. Monteiro ${ }^{1}$, J. Teer ${ }^{1}$, N. Woods ${ }^{1}$, H. McLeod ${ }^{1}$, E. Chao ${ }^{2}$, C. Radford $^{2}$, T. Pal ${ }^{1}$

1. Moffitt Cancer Center

2. Ambry Genetics

Introduction: A 56 year old Caucasian patient with prior history of non-medullary thyroid cancer at age 36 and 2 subsequent primary breast cancers tested negative for BRCA1, BRCA2 and PTEN mutations. She also tested negative for a panel of 14 breast cancer genes (BreastNext). Family history was significant for sister with follicular thyroid cancer at age 32 and melanoma at age 56. Methods: Germline DNA on the proband, her sister, her two unaffected brothers, and her parents was collected. Clinical grade full exome sequencing was performed on the proband and her parents, and segregation analysis on specific genes of interest was performed on her siblings. Full exome sequencing, bioinformatics analysis, filtering based on inheritance models, manual review with medical interpretation, and co-segregation analysis of candidate genes was performed. Results: Whole exome testing identified 112 potential genes. Post medical review, potential candidate genes and alterations were identified in the TSHR and STAT2 genes, with only the latter tracking with the phenotype. Consistent with patient's clinical history suggestive of Cowden syndrome, STAT2 interacts in the PTEN pathway. Specifically, the germline STAT2 variant of unknown significance (VUS) was c. $989 \mathrm{G}>\mathrm{A}$; this alteration is rarely observed in healthy cohorts, is highly conserved, predicted to be deleterious by in silico models, is located in the DNA binding domain, and possibly co segregated within this family. However, the likelihood that the STAT2 VUS is the cause of the patient's clinical symptoms remains uncertain. Discussion: Our findings suggest that STAT2 may be a newly identified inherited cancer susceptibility gene. Additional functional studies (including a DNA binding assay, quantitative PCR functional studies and signaling studies) are planned to further evaluate pathogenicity and clinical relevance of this finding.

\section{Diagnostic Exome Sequencing for Patients with a Family History of Consanguinity: Over 40 \% of Positive Results Do Not Follow an Autosomal} Recessive Pattern

\author{
Z. Powis ${ }^{1}$, K. Farwell Gonzalez ${ }^{1}$, C. Mroske ${ }^{1}$, L. Shahmirzadi ${ }^{1}$, D. El-Khechen ${ }^{1}$, C. Alamillo ${ }^{1}$, R. Baxter ${ }^{1}$, E. Chao ${ }^{1}$, S. Tang $^{1}$
}

\section{Ambry Genetics}

Diagnostic exome sequencing (DES) is an effective tool for diagnosis in intractable cases where the underlying cause is thought be genetic. Recent journals have numerous case reports of individuals with consanguineous family histories with rare genetic conditions identified by DES. It is commonly assumed that patients with a family history of consanguinity will have increased detection rates for rare autosomal recessive disorders through DES. Herein, we report the results from the patients referred to Ambry Genetics (Aliso Viejo CA) for DES with a reported consanguineous family history. Of the first 500 unselected cases referred for DES, $41(8.2 \%)$ had a known consanguineous family history. The degree of consanguinity varied from uncle and niece $(F=1 / 8)$, first cousins $(F=1 / 16)$, multiple loops of consanguinity, to "distant consanguinity." The degree of consanguinity in these families did not increase the likelihood of a positive exome result. Of patients with known consanguinity, $14(34.2 \%)$ had a positive result in a characterized gene, 5 $(12.2 \%)$ had an uncertain result, 3 (7.3\%) had a novel result and $19(46.3 \%)$ had a negative result. These results are not statistically significantly different from the overall breakdown of all patients for DES analysis. Of the positive results, 8 had homozygous mutations (ACAT1, ALS2, NEB, PDE6B, SPAST, TCIRG1, TMEM231, UBE3B) and $6(43 \%)$ had a molecular etiology unrelated to consanguinity. Four had alterations in autosomal dominant 
genes (VPS35, MPZ, CHD7, TRPS1) and two in X-linked genes (ARHGEF9 and SHROOM). Four of the mutations (CHD7, MPZ, ARHGEF9, TRPS1) arose de novo. All the alterations found in novel genes are inherited in an autosomal recessive fashion (DGK2, ZNF302, $S V 2 A)$. Although it was believed that individuals with known consanguinity would be more likely to have a positive result within an autosomal recessive gene, this did not prove to be the case. In summary, these results highlight that all inheritance patterns should be considered when testing patients with a known family history of consanguinity.

\section{Associations between Life Stage and Preferences regarding Return of Results from Whole Genome Sequencing: An Online Survey Study \\ E. Quinn ${ }^{1}$, S. Suckiel ${ }^{1}$, S. Sanderson ${ }^{2}$ \\ 1. Mount Sinai School of Medicine \\ 2. Icahn School of Medicine at Mount Sinai}

As whole genome sequencing (WGS) technology expands, it will likely be increasingly utilized in personalized medicine. Prior research suggests that individuals value the ability to make choices when learning WGS results. However, whether there is a correlation between life stage and the amount of information desired has not been explored. This study utilized an online questionnaire to collect opinions about personal WGS results from the general public $(N=275)$, and responses were stratified by gender, age, and parental status. Overall, participants expressed interest in learning personal WGS results and were observed to be most interested in learning about the categories of pharmacogenomics, actionable complex diseases, and carrier status and least interested in the categories of physical traits and unactionable complex neurological diseases. There were also significant differences between groups regarding the types and degree of information desired when learning these results; males desired information about a greater number of conditions than females, participants $>41$ yo were most likely to want to learn about neurological diseases, and non-parents were more interested in learning about carrier status than parents. Participants were more interested in learning their WGS results for actionable multifactorial conditions, such as Type 2 diabetes, than for unactionable multifactorial conditions, such as Parkinson's disease, and were more likely to share information about an increased genetic susceptibility for Type 2 diabetes with their children at younger ages than they would for Parkinson's disease. The findings of this study suggest that differences exist in the amount of information desired from WGS based on factors such as disease characteristics and life stage. Accordingly, these factors should be considered when delivering personalized WGS results.

\section{Interpretation and Clinical Implications of Significant Variants Detected via Whole Genome Sequencing in a Healthy Adult Cohort}

\section{E. $\operatorname{Ramos}^{1}$, J. Sardina ${ }^{1}$, A. Khouzam ${ }^{1}$, M. Hogue ${ }^{1}$, C. L. Mead ${ }^{1}$, S. Chowdhury ${ }^{1}$, T. Hambuch ${ }^{1}$}

1. Illumina

Clinical utility of whole genome sequencing is dependent on the interpretation of identified variants by experienced and qualified genetics professionals and the ability of the physician or genetic counselor to apply this information to the patient's medical management. The Illumina Clinical Services Laboratory has performed whole genome sequencing (WGS) of 381 predominantly healthy adults for the purpose of predisposition and carrier screening since May 2012. Here we present a summary of our findings and provide examples to illustrate the potential impact on medical management. For all individuals, variants in 1,600 genes associated with 1,221 monogenic conditions were evaluated for potential clinical significance. Evidence was evaluated by a team of geneticists and genetic counselors and variants were classified according to the American College of Medical Genetics and Genomics guidelines. Of the 381 individuals sequenced, approximately one-third had a variant that was pathogenic or likely pathogenic and expected to be clinically significant for the patient. Although pharmacogenomics and disorders with low penetrance and environmental influences were included in the assessment, conditions with significant clinical impact were identified including skeletal dysplasia, neurofibromatosis, cardiomyopathy, familial hypercholesterolemia and alpha-1 antitrypsin deficiency. Additionally, the vast majority of the cohort was a carrier for at least one variant suspected to be disease-causing, which could manifest in future generations or extended family. The findings in this patient cohort reinforce that WGS in healthy adults can impact clinical care and will require expertise on laboratory and clinical sides of testing. Challenges to the clinician include ascertaining the relevance of the variant based on the patient's clinical presentation, incorporating the results into symptom management or preventative strategies, and providing informed genetic counseling. A relationship with the team performing the variant assessment and reporting helps to support that effort.

\section{Parental Attitudes toward Whole Genome Newborn Screening}

\section{S. Rego ${ }^{1}$, M. Cho ${ }^{1}$, K. Ormond ${ }^{1}$}

\section{Stanford University}

Newborn screening exists for the purpose of testing newborns for medical conditions that can be severe, may be treatable, and are often not clinically evident at birth. The hypothetical use of whole genome newborn screening (WG NBS) as an alternative to the current NBS would dramatically expand the quantity and types of information parents could learn from screening and is likely to have both positive and negative implications. As WG NBS becomes more economical, it is probable it could be used in the future for the purposes of NBS. We conducted interviews with seven parents who had a child screen positive on NBS since August 2005 to assess their attitudes toward WG NBS; data was analyzed using a grounded theory approach. We found that study participants generally support the idea of WG NBS, all stating that they would elect to use it were they to have more children in the future. In particular, parents expressed strong interest in learning about medical conditions in their infant that were treatable or preventable. Several parents also wanted to know about untreatable and non-preventable conditions, usually citing a desire to be better prepared for the future. Parents who did not want information that was not actionable, particularly information about adult onset conditions, all believed such knowledge would be a burden. Age of onset was also important, with parents expressing much more interest in learning about conditions with childhood onset than adulthood. Despite supporting the implementation 
of WG NBS, parents had many concerns about potential implications of WG NBS on privacy, the time it would take for results to be returned and validated, the cost of the test, and the lack of knowledge most parents have about newborn screening. These results indicate that parents who have had a positive NBS result in the past may have more reservations and more limitations on the types of information they want from WG NBS than previous studies suggest.

\title{
A Trio-Based Whole Genome Sequencing Longitudinal Cohort Study: Experiences and Genetic Counselor Roles
}

\author{
S. Ruppert ${ }^{1}$, T. DeMarco ${ }^{1}$, B. Solomon ${ }^{1}$, D. Bodian ${ }^{1}$, W. Wong ${ }^{1}$, K. Huddleston ${ }^{1}$, R. Iyer ${ }^{1}$, R. Baveja ${ }^{2}$, R. Baker ${ }^{2}$, D. Ascher ${ }^{3}, J$. Vockley ${ }^{1}$, J. \\ Niederhuber ${ }^{1}$ \\ 1. Inova Translational Medicine Institute, Inova Health System \\ 2. Fairfax Neonatal Association, Inova Children's Hospital \\ 3. Department of Pediatrics, Inova Children's Hospital, Inova Health System
}

Thus far, questions related to the implementation and effects of large-scale genomic sequencing in healthcare have been approached in a largely theoretical manner. We have undertaken the largest longitudinal whole genome sequencing (WGS) study to date and describe our first year of experience and preliminary results that begin to address the gaps in methodology, resources, data analysis and management. The study team includes individuals with a wide variety of expertise, including clinical \& molecular geneticists, genetic counselors, bioinformatics scientists, clinical research specialists, and information technologists. Broad goals of the study include investigating the causes of a wide variety of conditions and determining best practices for prospective genomic medicine. The study focuses on WGS and related RNA/protein/epigenetic studies with a recruitment goal of 10,000 trio-based families. Since launch in 7/2012, >1,400 trios have been recruited and current accrual rate is $\sim 100$ trios/month, with a participation uptake rate of $\sim 35 \%$. Participating families enroll in early 2 nd trimester via 6 of our obstetric clinics. Longitudinal follow up is anticipated to continue into adulthood. All data and analysis are cloud-based and standard medical information is captured in a single electronic medical record system and later combined with studyspecific clinical and other data. The direct cost of WGS and other 'omic data generation is $\sim \$ 4,000$ per individual participant; there are many indirect costs. We have constructed novel, population-specific algorithms for variant filtering based on sub-population allele frequency within our diverse cohort. Analytic power is increased by leveraging the combined data from this study and from several of our other, separate large-scale genomic studies. The complexities involved in consent, variant interpretation and return of results present unique opportunities for genetic counselors to be intimately involved in many aspects of study planning \& implementation and to contribute to shaping the future of this type of genomic research.

\section{0,000 Cases and Counting: The High Volume Clinical Experience with Noninvasive Prenatal Testing}

J. S. Saldivar ${ }^{1}$, T. Boomer ${ }^{1}$, J. Jesiolowski ${ }^{1}$, J. Wardrop ${ }^{1}$, R. McCullough ${ }^{1}$, R. Crabb ${ }^{1}$, P. Oeth ${ }^{1}$, W. Paxton ${ }^{1}$, N. Dharajiya ${ }^{1}$, T. Monroe ${ }^{1}$, A. Bombard $^{2}$

1. Sequenom Laboratories

2. Sequenom Inc.

Introduction: Maternal age, positive serum screening, ultrasound abnormalities, or family history increase the risk for trisomy 21 and other aneuploidies. Amniocentesis and chorionic villus sampling are widely used surgical procedures but have a small risk of complications including miscarriage. The adoption of a highly accurate, noninvasive screening test (NIPT) has been rapid, resulting in a paradigm shift in the management of these patients, and has now been in clinical use for almost 3 years. Methods: A noninvasive aneuploidy test using massively parallel sequencing of cell-free DNA from maternal blood was developed by Sequenom Laboratories and validated in blinded clinical studies with more than 6,000 samples. In addition, upwards of 250,000 patients have been tested over a 30 month period using the MaterniT21 PLUSTM test with the solicited follow-up from physicians regarding test outcome. Herein we describe our experience with first 185,000 samples run with the latest version of our analytical pipeline. Results: The predominant indication for testing remains age-related risk, followed by positive serum screening and ultrasound findings. Overall positivity rates were $1.48 \%, 0.45 \%$, and $0.2 \%$ for chromosomes 21,18 , and 13 , respectively. Approximately $4.1 \%$ of cases were designated as multifetal. Average turnaround time was 5.2 calendar days, and the test had a total non-reportable rate of $1.4 \%$. Estimated analytical performance based on partial ad hoc clinical outcome show that sensitivity and specificity are similar to that found in the original tightly controlled clinical validation studies. Conclusion: NIPT offers high-risk patients accurate and reliable information about fetal aneuploidy status. This test has demonstrated stable positivity rates for trisomy 21, 18 and 13, which mirror those found in large invasive studies on high-risk populations. Operational performance demonstrates a robust and efficient process, and the estimated analytical performance assessment concurs with expectations from the original clinical validation studies.

\section{Homozygous Mutations in Newly Described Gene, CNTN?AP1, Detected through Whole Exome Sequencing Provide Diagnosis for Siblings}

\author{
A. Shealy ${ }^{1}$, N. Friedman ${ }^{2}$, P. Liu ${ }^{3}$, Y. Yang ${ }^{3}$, R. Moran ${ }^{1}$ \\ 1. Genomic Medicine Institute, Cleveland Clinic \\ 2. Neurological Institute, Cleveland Clinic \\ 3. Department of Molecular and Human Genetics, Baylor College of Medicine
}

Introduction: Mutations in $C N T N A P 1,17 \mathrm{q} 21.2$, were very recently linked to distal arthrogryposis multiplex congenita, severe hypotonia, and axoglial defects. Here we report on 2 affected siblings. For one of them whole exome sequencing was performed; homozygous mutations in CNTNAPI were detected. Case Report: A consanguineous couple has had 4 pregnancies complicated by polyhydramnios and reduced fetal movement. One ended in a late intrauterine fetal death and 3 infants died from respiratory failure due to severe hypotonia. We have evaluated 2 of these children; both boys, $\mathrm{M}$ and $\mathrm{Y}$, had severe hypotonia, respiratory failure, and minor dysmorphic features. Additionally, $\mathrm{M}$ had clubfeet and hyperpigmented macules; $\mathrm{Y}$ had correctable distal arthrogryposis, areflexia, and mild micrognathia. On single nucleotide polymorphism (SNP) array, both had a block of loss of heterozygosity (LOH) from 17q12q21.33. An EMG was performed on Y and suggested a diffuse motor axon loss disorder. Whole exome sequencing was performed on $\mathrm{Y}$ and a homozygous nonsense mutation in CNTNAPl was found. Both parents were confirmed to be heterozygous. Discussion: Only 5 other cases of CNTNAP1 mutations have been reported. All died by 80 days of age and were reported to have severe hypotonia, distal arthrogryposis multiplex congenital, axoglial defects, areflexia, and respiratory failure. Our 2 cases have symptoms that were consistent with previously reported cases. However, our cases also had some dysmorphic features, which may represent an expansion of the phenotype. Because it was not available at the time of death, $\mathrm{M}$ 
did not have whole exome sequencing; however because of LOH on SNP array which includes CNTNAP1 and because of the consistency of features, we assume his diagnosis is the same. Testing of CNTNAP1 should be considered when there is polyhydramnios along with severe hypotonia, distal arthrogryposis, and axoglial defects. Additionally, this case may serve as a basis for future comparison, providing some anticipatory guidance for use in future genetic counseling cases in which mutations within, or deletions of, CNTNAP1, are found.

\title{
Reporting Incidental Findings in Clinical Whole Exome Sequencing: Incorporation of the 2013 American College of Medical Genetics and Genomics Recommendations into Current Practices of Genetic Counseling
}

\author{
L. Smith ${ }^{1}$, J. Douglas ${ }^{2}$, A. Braxton ${ }^{3}$, K. Kramer ${ }^{1}$ \\ 1. Brandeis Univeristy \\ 2. Boston Children's Hospital \\ 3. Baylor College of Medicine Medical Genetics Testing Lab
}

In March 2013, the American College of Medical Genetics and Genomics (ACMG) released recommendations for reporting incidental findings (IFs) from clinical whole genome and exome sequencing (WGS/WES). These recommendations generated considerable debate among members of the genetics community regarding medical beneficence and patient autonomy. The purpose of this study was to investigate how the ACMG recommendations for reporting IFs have influenced current practices of genetic counselors. We invited genetic counselors actively involved in offering WES for clinical diagnosis to participate in an online, anonymous survey. Forty-six respondents recruited through the NSGC listserv completed the survey. Thirtyfour of the respondents were in practice prior to the March 2013 recommendations, and of those, $62 \%$ reported that the amount of time they spent counseling for IFs had increased. However, most of the respondents (76\%) did not feel that their sessions had become more challenging as a result of the recommendations. Approximately $50 \%$ of the respondents indicated that they report all IFs, regardless of patient age, while one third consider factors such as age and parent preference in reporting IFs. Approximately $40 \%$ of respondents reported that the testing laboratory's policy for returning IFs has an influence on laboratory selection; of those, $72 \%$ indicated that the option to decline receipt of IFs has a significant influence on laboratory selection. Most respondents (94\%) reported that patients generally want to receive IFs. However, some respondents noted that they have encountered patients who wished to decline receipt of IFs. The ACMG has addressed this issue recently by updating their recommendations to reflect the importance of patient preference with respect to IFs. Our study provides empiric data for the ongoing discussion regarding disclosure of IFs from WES. Further investigation into the opinions and practices of additional provider populations as well as those of patients would be valuable.

\section{Genotype-Phenotype Correlation with Exome Sequencing}

\section{E. Smith ${ }^{1}$, A. Mani ${ }^{1}$}

\section{Yale University School of Medicine}

The Yale Program for Cardiovascular Genetics (YPCG) uses whole exome sequencing (WES) as a first line test for mutation screening in patients with cardiovascular single gene disorder of unknown cause. Testing is performed after careful phenotyping and clinical evaluation by referring providers as well the genetics team. Two women of Caucasian background were referred to YPCG for genetic testing and risk assessment for Long QT syndrome (LQTS). Both individuals had aborted sudden cardiac arrest (SCA) and an abnormal electrocardiogram (EKG) with prolonged QT duration. Both had undergone implantable cardioverter defibrillator implantation post-arrest. Neither had significant family history of sudden death or arrhythmias. WES was performed in both cases; no mutation was identified in genes associated with LQTS or other cardiac arrhythmias. Reexamination of medical records revealed that both cases had echocardiographic diagnosis of cardiomyopathy. Case 1 had echocardiographic findings consistent with restrictive cardiomyopathy and a splice-site altering mutation in $T N N I 3$, known to cause restrictive cardiomyopathy. Case 2 had a mildly dilated left ventricle with mildly reduced ejection fraction suggestive of dilated cardiomyopathy. She had a frameshift mutation in TTN and a missense mutation in MYH6. The information revealed by WES, complemented by reexamination of the medical records had significant impact on the care of patients and relatives, including risk assessment, clinical evaluation, genetic screening, and potential treatments. These cases demonstrate the benefits and challenges of utilizing WES as a first line test. Pretest counseling focused on determining a causal variant for LQTS, disregarding other potential causes. Post-test counseling, therefore, had to include appropriateness of diagnosis of cardiomyopathy based on WES, review of the medical records, reexamination of the patients, and their EKG. Counseling also had to take into account the impact of this new information on the medical management of the patients and relatives.

\section{Importance of Clinical Data in the Interpretation of a Novel EHMT1 (Kleefstra Syndrome) Variant Identified by Whole Genome Sequencing}

\section{B. Smith-Packard ${ }^{1}$, J. Williams ${ }^{1}$, T. Challman ${ }^{1}$, C. Martin $^{1}$, M. Williams ${ }^{1}$}

\section{Geisinger Health System}

Introduction: Kleefstra syndrome is characterized by intellectual disability, childhood hypotonia, expressive speech delay and characteristic facial features. This autosomal dominant condition is typically de novo and caused by microdeletions at 9q34.3 (75\%) or mutations in the EHMT1 gene ( $25 \%$ ). Here we present a patient found to have a novel, de novo mutation of EHMT1 identified through our whole genome sequencing (WGS) clinical research program and the process used for clinical interpretation of this finding. Case Report: A 7 year-old female was referred to our WGS program following a history of extensive clinical evaluations and genetic testing. Her mother has adult-onset seizures of unknown etiology. Her complex medical history includes intellectual disability, hypotonia, sleep disturbance, recurrent pneumonia, depigmented skin patches, and dysmorphic features. The patient's differential diagnoses included Smith-Magenis syndrome, Rett syndrome, and tuberous sclerosis. Previous testing included a G-banded karyotype, genome-wide chromosomal microarray, MECP2 and RAIl sequencing, chromosome 15 methylation analysis, plasma and urine amino acids, echocardiogram, brain MRI and EEG. WGS identified a novel, de novo variant in EHMT1 that results in a codon deletion in exon 26 (p.Phe1227del; c3679_3681 del). The mutation was characterized by the testing laboratory as being of uncertain significance and was confirmed by capillary sequencing. Discussion: Analysis of this variant found that it is located at a moderately conserved protein residue within the conserved SET domain of EHMT1 and is absent from control population datasets. Further investigation of the Kleefstra phenotype led to review of parent association websites and confirmed that other individuals with Kleefstra exhibit depigmented patches, a finding that has not been previously noted. Combining the laboratory data with selfreport information from families, detailed clinical information from the referring physician, and expert help from Dr. Kleefstra led to assigning the variant p.Phe1227del as pathogenic. 


\title{
Motivation and Consumer Willingness for Self-Pay Noninvasive Prenatal Testing at the Medcan Clinic in Canada
}

E. Stephenson' ${ }^{1}$, J. Davies ${ }^{2}$

1. Sarah Lawrence College

2. Medcan Clinic

Noninvasive prenatal testing (NIPT) was introduced into the Canadian healthcare system by the Medcan clinic in December of 2012. NIPT is the first self-pay prenatal test available to Canadian women, as most other prenatal services are covered by provincial health insurance. This study examined the inquiries for NIPT at Medcan during the first year of service, in order to develop an appreciation of consumer willingness, motivation and uptake. To date, there are no other studies examining patient motivation and interest for a self-pay prenatal test in Canada. Using a patient inquiry log that was compiled by Medcan staff from December 2012 through December 2013, patient data was collected for all inquiries $(n=291)$. This included patient demographics like maternal age, gestational age and province of residence, as well as other pertinent information like referral source and testing indication. This study suggests that pregnant Canadian women that are interested in learning about NIPT are motivated to seek out and complete testing. Reasons for choosing NIPT may vary among women, but in this study, factors like the referral source, indication and gestational age all affected the rate of uptake. Results from this study may be useful for other Canadian clinics looking to offer NIPT. An understanding of who is interested in this technology, and how they learn about it, can inform other clinics and laboratories about how to reach and inform patients that may benefit from NIPT. This information may also help to inform policy decisions in Canada regarding plans for provincial coverage and implementation.

\section{Significance of Reporting Candidate Genes with a Potential Relationship to a Disease Phenotype Identified by Whole Exome Sequencing}

\section{J. Tahiliani ${ }^{1}$, P. Vitazka ${ }^{1}$, J. Juusola ${ }^{1}$, B. Friedman ${ }^{1}$, J. Neidich ${ }^{1}$, N. Smaoui ${ }^{1}$, K. Retterer ${ }^{1}$, S. Bale ${ }^{1}$, E. Haverfield ${ }^{1}$}

1. GeneDx, Inc.

Whole exome sequencing (WES) is an effective diagnostic tool for the identification of the molecular basis of human genetic disorders. WES associates a patient's clinical phenotype to specific genes by evaluation of approximately 20,000 genes in the human genome. With the increased rate of publications of mutations in newly described genes, it is important to assess genes associated with human genetic disease. The significance of sequence changes identified by WES can be addressed by databases such as the Human Gene Mutation Database (HGMD $\left.{ }^{\circledR}\right)$, which contains disease association information on about 5,700 genes. WES also identifies new potential genetic etiologies that have not been linked with human genetic diseases (candidate genes). The clinical utility and research potential of reporting out sequence changes in candidate genes has yet to be established. Since 2012, approximately $16 \%(601 / 3,699)$ of the total number of reported sequence changes by WES at GeneDx, Inc. have been in candidate genes. Findings reported in candidate genes were retrospectively reviewed to determine whether any new information linking these genes to a genetic disorder has become available since initially reported. These candidate genes were crosschecked against HGMD to assess how many of these genes had been newly added to the database since the initial report. Of these candidate genes, $3 \%(13 / 431)$ have since been reported in HGMD with an association with a genetic disorder. This result suggests there is value to reporting findings in candidate genes. Reevaluation of candidate genes on a periodic basis may be helpful in clarifying the cause of a phenotype with unknown genetic etiology. With this information, genetics professionals must counsel patients about how genetic information is improving over time as a component of the benefits and limitations of WES. Ongoing communication and collaboration between genetic professionals and genetic testing laboratories is important to maximize the benefits of the existing technology and ensure appropriate genomic counseling is provided.

\section{Clinical Experience Reporting Trisomy 16 And 22 On Noninvasive Prenatal Testing: Test Performance and Implications for Genetic Counseling}

\author{
N. Teed ${ }^{1}$, T. Boomer ${ }^{2}$, J. Jesiolowski ${ }^{2}$, J. Wardrop ${ }^{2}$, N. Dharajiya ${ }^{2}$, T. Monroe ${ }^{2}$, J. S. Saldivar ${ }^{2}$ \\ 1. Sequenom, Inc. \\ 2. Sequenom Laboratories
}

Background: Most clinical experience with noninvasive prenatal testing (NIPT) has focused on common autosomal trisomies $(21,18,13)$ and sex chromosome aneuploidies. In October 2013, Sequenom Laboratories expanded reporting for the MaterniT21TM PLUS laboratory-developed test to include trisomies 16 (T16) and 22 (T22). These trisomies are among the most common aneuploidies at conception but typically result in early miscarriage. Methods: NIPT using massively parallel sequencing of cell-free DNA from maternal blood was performed by Sequenom Laboratories. In this observational case series, all pregnancies reported positive for T16 and T22 through the CLIA laboratory were cataloged and follow-up was obtained. Results: Since reporting of additional trisomies began, 75,977 samples have been resulted by the laboratory. Of those, 23 were positive for T16, and 20 were positive for T22. Of these, 6 were confirmed as abnormal by diagnostic testing, 18 were likely positives based on clinical history, 14 were pending outcome, and 5 were discordant. Confirmed or likely findings of confined placental mosaicism (CPM), including those resulting in intrauterine growth restriction, as well as ongoing pregnancies with identified congenital anomalies were reported in this cohort. Conclusions: Positive results for T16 and T22 have been reported at expected incidence rates. Ultrasound examination, serum biochemistry results, and confirmatory diagnostic testing provide additional context for the interpretation of NIPT results. Confirmed results and/or concordant clinical history provide an explanation for fetal loss or abnormal ultrasound findings. Discordant results may prompt additional clinical monitoring and conservative pregnancy management. Complex counseling needs may arise and collaboration between the referring provider, a high-risk center, and the performing laboratory is essential.

\section{Opt-Out Rates for Receiving Secondary Findings from Whole Exome Sequencing: The Experience of a Clinical Laboratory}

A. Telegrafi ${ }^{1}$, K. Retterer ${ }^{1}$, B. Friedman ${ }^{1}$, A. Daly ${ }^{1}$, A. Fuller ${ }^{1}$, J. Tahiliani ${ }^{1}$, H. Hanson Pierce ${ }^{1}$, D. Copenheaver ${ }^{1}$, J. Juusola ${ }^{1}$, P. Vitazka ${ }^{1}$, N. Smaoui $^{1}$, J. Neidich ${ }^{1}$, S. Bale ${ }^{1}$, E. Haverfield ${ }^{1}$

1. GeneDx

The American College of Medical Genetics and Genomics (ACMG) issued recommendations in March, 2013 for the return of results from clinical genome-scale sequencing to include reporting of incidental findings (IF) on all individuals who receive clinical whole exome sequencing (WES) or whole genome sequencing (WGS), irrespective of the patient's age and right not to know. The recommendations stressed the importance of actively analyzing 56 carefully chosen genes for known or expected pathogenic mutations, regardless of clinical indication for testing, that could indicate the presence or risk for 24 disorders for which early intervention is likely to reduce or prevent serious morbidity or early mortality. ACMG has since issued 
updated recommendations in March 2014 to allow that patients should have the right to opt-out of the analysis of IF when the sample is sent for clinical WES or WGS. At GeneDx, patients have been offered the option to opt-out of the analysis of the 56 recommended genes since July 1 , 2013. We performed a retrospective review of the consent forms received between July 1, 2013-April 30, 2014 to evaluate the opt-out rates. From 2,394 unrelated probands who underwent WES during this period, 549 (23\%) opted-out of receiving ACMG IF and 1,845 (77 \%) had the recommended 56 genes evaluated as part of WES. Of the 822 completed and reported cases that have requested IF, we have identified IF in 55 (6.7 \%) cases. In order to identify factors that affect opt-out rates and help inform the consenting process in the future, we will assess differences in consent opt-out rates based on gender, ethnicity, referring subspecialty and compare the rate in parents of affected children versus that in affected adult probands. Although the current analysis may not be reflective of the preferences in the general population, it represents a subset of patients that prefer to opt-out from receiving IFs. These data provide preliminary support to the update issued by ACMG where patient preferences are honored.

\title{
Successful Utilization of Enhanced Exome Sequencing to Identify the Genetic Cause Of Retinal Disorders in a Case Series
}

\author{
J. Tirch ${ }^{1}$, M. J. Clark ${ }^{1}$, S. Strom ${ }^{2}$, A. Martinez ${ }^{3}$, S. Garcia ${ }^{1}$, G. Chandratillake ${ }^{1}$, J. Harris ${ }^{1}$, A. Patwardhan ${ }^{1}$, S. Chervitz ${ }^{1}$, M. Li ${ }^{1}$, M. Pratt ${ }^{1}$, G. \\ Bartha $^{1}$, S. Luo ${ }^{1}$, R. Chen ${ }^{1}$ \\ 1. Personalis, Inc. \\ 2. Department of Pathology and Laboratory Medicine, David Geffen School of Medicine at University of California, Los Angeles \\ 3. University of California, Los Angeles Jules Stein Eye Institute
}

Identifying the genetic etiology for retinal disorders is challenging because of allelic, phenotypic, and locus heterogeneity, as well as environmental toxicities resulting in phenocopies. Patients often endure long diagnostic odysseys involving many single gene and/or gene panel tests. Whole exome sequencing (WES) is a highly appealing alternative to panels, which require frequent revision as new causative genes are discovered; however, incomplete coverage of relevant genes means standard WES is also non-ideal. To address these limitations, we developed the Accuracy and Content Enhanced (ACE) Exome, which improves sensitivity to detect variants by enhancing coverage over genes of biomedical relevance. We conducted ACE Exome sequencing for members of eleven families with undiagnosed retinal disorders and used a novel automated system to rank variants by integrating family history and phenotypic information with the exome data. For ten of these families, we successfully identified the genetic basis of their retinal disorder. Several of these diagnoses would have been either missed completely or timely and expensive to pick up via sequential gene testing protocols due to the involvement of genes that are thought to rarely cause disease (e.g., variants in $C R X$, which is associated with only $1 \%$ of cases of retinitis pigmentosa (RP), were detected in three of our families). Three diagnoses involve genes not currently present on gene panels available in the US: one family has a novel homozygous variant in LEPREL1, a gene that has only been associated with retinal disorders in a single family in the literature; the second involves NMNAT1, another recently described gene available only as a single gene test; the third family has a variant in a new gene, previously considered a candidate gene for RP. In addition, one family has an X-linked etiology that would have been missed by standard exome sequencing due to poor gene coverage of $R P G R$. These successes demonstrate the efficacy of enhanced exome sequencing to diagnose the genetic cause of retinal disorders.

\section{Parents' Perspectives: Child's Whole Exome Sequencing Research Results of Uncertain Significance}

G. Tran' ${ }^{1}$, A. Antommaria ${ }^{2}$, R. Pickler ${ }^{2}$, K. Sund ${ }^{2}$, C. A. Prows ${ }^{2}$

1. Genetic Counseling Program, University of Cincinnati

2. Cincinnati Children's Hospital Medical Center

Introduction: Whole exome sequencing (WES) is becoming a common tool used in both research and clinical settings. Stakeholders' attitudes regarding disclosure of research results are variable and a better understanding of participants' preferences is needed. Contradictory evidence exists regarding adult participants' comprehension and perceived impact of disclosed results of uncertain significance. There is also limited literature discussing how parents make sense of WES research results of uncertain significance. Purpose: To explore parents' understanding of, reactions to, and ascribed meanings of WES research results of uncertain significance. Methods: The investigators conducted semi-structured interviews with eight parents of five children who received results of uncertain significance. The investigators used an interpretive phenomenology approach to analyze the data. They developed codes using deductive and inductive logic and two coders coded all transcripts. Results: Parents fluctuated in their understanding of their child's WES research results of uncertain significance. Parents expressed disappointment and lingering uncertainties with disclosed results. Parents still had positive views regarding the research results because they saw it as an opportunity to benefit other children/families. In general, parents were willing and wanting to share their child's results with friends and family members. Parents had high hopes for continuing research on their child's results. Conclusion: Genetic providers will need to evaluate the efficacy of current strategies in explaining results of uncertain significance and may need to develop educational tools. Alternatively, genetic professionals have to better address underlying reasons for parents' fluctuating interpretations of research results. Further studies are needed to assess how receipt of research results of uncertain significance can impact parents' psychosocial health. Future studies are needed to assess the potential impacts decreased funding of future research on participant recruitment.

\section{Application of Risk-Score Analysis to Low-Coverage Whole Genome Sequencing Data for the Noninvasive Detection of Trisomy 21 and Trisomy 18}

\section{J. Tynan ${ }^{1}$, S. Kim ${ }^{1}$, A. Mazloom ${ }^{1}$, C. Zhao ${ }^{1}$, J. Torres ${ }^{1}$, M. Ehrich ${ }^{1}$}

1. Sequenom, Inc.

Introduction: Whole genome massively parallel sequencing of cell free DNA in maternal plasma has been established in noninvasive prenatal testing as the most comprehensive testing modality to date. While some medical providers value the binary result given today, other medical providers prefer to communicate a patient's risk for carrying an affected fetus, especially in the context of a general population screening test. Here we present results from a new assay, specifically designed to reduce testing complexity and decrease cost, which generates a risk score result for trisomy 21 and trisomy 18 . Methods: Sequencing results were generated from 352 samples including 25 trisomy 21 samples, and risk score was calculated from the chromosome proportion based risk. A risk score based on chromosome proportion was determined from a probability calculation conditional on fetal fraction, chromosome dosage of a euploid population, chromosome dosage of an expected trisomic population, and a maternal age dependent prior probability. A risk score threshold of 1 in 100 was used to stratify samples into high or low risk categories. Sensitivity and specificity were calculated using this 
stratification method. Results: We performed in silico modelling based on low coverage whole genome sequencing data. The simulation results indicate that a risk score based method could provide sensitivity greater than $99 \%$ for trisomy 21 and trisomy 18 detection. Our preliminary results indicate that detection of trisomy 21 could be consistent with expectation. Results from trisomy 18 testing were not available at the time of submission but will be included if available at the time of presentation. Conclusion: We conclude that a risk score based method coupled with a modified test format is highly sensitive and specific for trisomy 21 screening. Such a test provides an improved performance relative to current serum screening, in a result format associated with risk reporting of the last 30 years.

\title{
Understanding Next Generation Sequencing Results: An Update on Elements of Variant Interpretation and Classification
}

\author{
K. Vikstrom ${ }^{1}$, B. Kirkpatrick ${ }^{2}$, L. Catalano ${ }^{3}$, K. Nyhamp ${ }^{4}$, S. Topper ${ }^{4}$, M. Powers ${ }^{4}$, M. Anderson ${ }^{4}$ \\ 1. Invitae Corporation \\ 2. Geisinger Health System \\ 3. Emory Genetics Laboratory \\ 4. Invitae Corporation
}

With the growing acceptance and utilization of next generation sequencing (NGS) techniques for diagnostic purposes, the complexity and uncertainty of the findings are also expected to increase. Understanding the process by which a diagnostic laboratory analyzes and classifies sequence variants has clinical utility for genetic counselors, as patients need to be informed of the rationale behind variant interpretations, particularly in cases where a variant of unknown significance is found and may be reclassified in the future. The authors present an overview of the standard, evidence-based practice of scoring and classifying variants, as proposed by the most recent American College of Medical Genetics and Genomics (ACMG) guidelines (March 2013). Examples of sequence variant interpretations, across all proposed classifications (i.e. pathogenic, likely pathogenic, uncertain significance (VUS), likely benign and benign) will be presented as a way to highlight important aspects of the interpretation process. A discussion of the variant interpretation process will include the following: 1) Sources of evidence for the gene/disease causality and interpretation of a specific variant (PubMed, ClinVar, HGMD, LOVDs, ESP, $1 \mathrm{~K}$ Genomes, etc.), and the limitations of these resources; 2) Models and tools used to determine the impact of sequence change on protein structure (e.g., Polyphen, SIFT); 3) Examples of scoring and classifying a variant based on available evidence as well as data necessary for reclassification.

\section{Detection of Maternal 22q Deletions by Noninvasive Prenatal Testing}

\author{
J. Wardrop ${ }^{1}$, S. Weber ${ }^{2}$, D. Farkas ${ }^{3}$, E. Almasri ${ }^{1}$, C. Zhao ${ }^{1}$, C. Deciu ${ }^{1}$, T. Boomer ${ }^{1}$, J. S. Saldivar ${ }^{1}$, N. Dharajiya ${ }^{1}$ \\ 1. Sequenom Laboratories \\ 2. Geisinger Health System \\ 3. Sequenom Laboratories
}

Introduction: Fetal aneuploidies can now be detected noninvasively with high sensitivity and specificity through interrogation of circulating cell-free DNA. Initially covering full chromosomal gains of $21,18,13$, noninvasive prenatal testing (NIPT evolved to analyze sex chromosomal aneuploidies. The whole genome sequencing approach enables the opportunity to report on additional regions of the genome. We have extended the content of the MaterniT21TM PLUS test to include subchromosomal deletions. Here we report four cases of maternal 22q deletions detected by NIPT, two of which were previously undiagnosed. Methods: Maternal plasma samples were subjected to DNA extraction, library preparation, and massively parallel sequencing as previously described. Sequencing data were analyzed using a novel algorithm to detect microdeletions. Sequenom laboratories has processed $>75,000$ samples using this extended tool. Results: The first case was positive for a 22q deletion. Microarray analysis of maternal blood, revealed a $2.54 \mathrm{Mb}$ loss at 22q11.21 providing a primary diagnosis. The patient had seizure disorder, learning disabilities, low calcium and dental problems. The fetal deletion has not yet been confirmed. The second case involved a twin gestation, NIPT was positive for a $22 \mathrm{q}$ deletion. Invasive testing and microarray analysis revealed that one fetus and the mother carried the 22q deletion. Clinically, the mother was noted to have developmental delay and a hyernasal voice. In both cases, NIPT established the primary diagnosis. For two cases of $22 \mathrm{q}$ deletion, the maternal diagnosis was known before NIPT, and was confirmed by our analysis. Additionally, 8 reported fetal deletions have been confirmed by invasive testing or are concordant with clinical findings. Conclusion: The detection of $22 q$ deletions demonstrates the capabilities of a whole genome approach to noninvasive testing. Establishing maternal 22q deletions by NIPT further augments the clinical utility of a whole genome sequencing approach. These examples show the potential of NIPT, in providing microarray-like testing in the future.

\section{Secondary PTEN Mutation Identified on Whole Exome Sequencing}

C. Williams ${ }^{1}$, P. Arscott ${ }^{1}$, V. Raymond ${ }^{1}$, T. Else ${ }^{2}$, S. Day ${ }^{2}$

1. University of Michigan

2. University of Michigan Health System

Introduction: Increasing use of whole exome sequencing (WES) has tasked medical professionals with disclosure of secondary findings, possibly associated with conditions in which they do not specialize. This case highlights the value of inter-specialty genetic counselor (GC) collaboration in secondary finding disclosure. Case Report: A 51 year-old male with left ventricular noncompaction (LVNC) diagnosed at age 48 presented to an inherited cardiomyopathy clinic for evaluation. His family history was notable for a son diagnosed with LVNC in his 20s, but was otherwise negative for LVNC or evidence of an inherited cancer syndrome. Following genetic risk assessment and counseling, a clinical dilated cardiomyopathy panel was ordered and was negative for deleterious sequence mutations. Follow-up clinical WES of the proband, his parents, and affected son was ordered, and did not identify a definitive explanation for the LVNC phenotype. However, a secondary, known pathogenic germline PTEN mutation (c.517C $>$ T) was identified in the proband and his son consistent with a diagnosis of PTEN Hamartoma Tumor syndrome (PHTS). The proband's parents were negative for the PTEN mutation. The ordering GC contacted a cancer genetics GC to review results, discuss implications and coordinate follow-up. The ordering GC disclosed the result and referred the proband and his son to the cancer genetics GC for full PHTS evaluation and coordination of management. Physical exams for both the proband and his son were notable only for macrocephaly $(63.5 \mathrm{~cm}$ and $66.0 \mathrm{~cm}$ respectively; $>98 \mathrm{th}$ centile). PTEN genetic testing is pending in the proband's siblings and other children. Discussion: Secondary findings in clinical WES are anticipated and can be managed appropriately 
through inter-specialty collaboration, as evidenced by this case. These collaborations enable skilled disclosure by the ordering GC who may not have expertise in the condition indicated by the finding, patient follow-up in an appropriate clinic, and cascade genetic testing for the family.

\section{Psychological Impact of Receiving Secondary Findings from Whole Exome Sequencing}

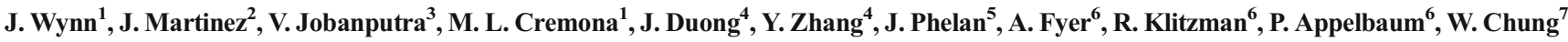

1. Department of Pediatrics, Columbia University Medical Center

2. Columbia University Medical Center

3. Department of Pathology, Columbia University Medical Center

4. Department of Biostatistics, Mailman School of Public Health, Columbia University

5. Department of Sociomedical Sciences, Mailman School of Public Health, Columbia University

6. Department of Psychiatry, Columbia University Medical Center

7. Department of Medicine and Pediatrics, Columbia University

Introduction: Whole exome sequencing (WES) is now commonly used in research and clinical care. It has the capacity to identify genetic information unrelated to the primary indication, i.e., secondary findings. There has been concern that disclosing secondary findings, especially when associated with risk of disease to the individual, could result in a negative psychological impact. Little research has been conducted on the psychological impact of receiving secondary findings. We examined the psychological impact of receiving secondary findings from WES. Methods: Adults who had previously participated in research studies utilizing WES were given the option of receiving secondary findings. Participants could choose which type(s) of secondary findings they wanted to receive and results were disclosed in a follow-up session after CLIA confirmation. Qualitative data were collected and validated scales to assess anxiety (Beck Anxiety Inventory (BAI)) and depression (Personal Health Questionnaire-9 (PHQ-9)) were administered at enrollment and 1 month following results disclosure. Changes in anxiety and depression were compared between participants who did and did not receive results that had a potential health impact. Results: Sixteen participants received results with a potential health impact (group 1) and 28 (group 2 ) did not. Group 1 had a mean increase in the $\mathrm{BAI}$ of $+0.56, \mathrm{SD}=7, p=0.7$ and group 2 had a mean decrease of $-0.36, \mathrm{SD}=4, p=0.6$. The mean decrease in PHQ-9 of group 1 was $-0.56, \mathrm{SD} 2, p=0.25$ and for group 2 it was $-0.86, \mathrm{SD}=2, p=0.05$. When comparing the change in the scales between the two groups, there was no significant difference in the BAI $(p=0.63)$ or in the PHQ-9 $(p=0.64)$. Conclusion: Contrary to concerns about the impact of returning secondary findings, we did not find an adverse effect on anxiety or depression for participants who received secondary findings, even for results with a potential health impact. Our results may have implications for policies being developed regarding returning secondary findings and for predictive genomic testing.

\section{Perceived Obligations by Researchers in Genetics to Return Individual Research Results and Incidental Findings to Participants}

\section{Young ${ }^{1}$, B. Biesecker ${ }^{2}$, J. Bollinger ${ }^{3}$, R. Dvoskin ${ }^{3}$, D. Kaufman ${ }^{3}$}

1. Johns Hopkins University, National Human Genome Research Institute, National Institutes of Health

2. Genetic Counseling Training Program, Johns Hopkins University/National Human Genome Research Institute

3. Genetics and Public Policy Center

Genetics researchers increasingly face decisions about returning individual research results (IRRs) and incidental findings (IFs) to participants. Prior data suggests that participants are interested in receiving such results, and there has been vigorous debate in the genetics community about the extent of researcher obligation to return IRRs and IFs. However, little research has focused on whether the researchers themselves feel such an obligation. As whole genome and exome sequencing (WGS/WES) becomes more widely used in research, and in light of the recent American College of Medical Genetics and Genomics (ACMG) recommendations about return of IFs in clinical WGS/WES, it is likely that an increasing number of researchers will be confronted with deciding whether to return IRRs and IFs. Thus, individual researcher preferences for the return of results will play a key role in implementation. A survey of U.S. human genetics researchers was conducted by the Genetics and Public Policy Center to examine researcher perceptions of legal and ethical obligation to return IRRs and IFs. The survey was completed by 358 researchers. The majority (62\%) work in a university/academic setting, and most are males $(63 \%)$. The majority $(68 \%, n=242)$ support returning IRRs or IFs to participants, yet less than half reported feeling an obligation to return results (IRRs: $44 \%, n=158$; IFs: $44 \%, n=157)$. Most researchers $(78 \%, n=279)$ reported no obligation to look for medically significant variants unrelated to the focus of their research. The majority of respondents $(60 \%)$ do not return any results. There was a relationship between feelings of obligation and return of results. For example, $58 \%$ of those who felt some obligation to return IFs may return them, while only $24 \%$ of those who did not feel obligated returned IFs. Overall, researchers are divided both in their perceived ethical obligation to return genetic results and in their practice of actively returning IRRs and IFs. The correlation between obligation and reported practice is evidence for the relationship of individual preferences and decisions whether to return IRRs and IFs.

\section{Genetic Testing II}

\section{Determining the Clinical Significance of $B R C A 1$ and $B R C A 2$ Intronic and Exonic Splicing Variants}

\section{K. Bowles ${ }^{1}$, N. Singh ${ }^{1}$, U. Kappes ${ }^{1}$, E. Mundt ${ }^{1}$, B. Warf ${ }^{1}$, B. Morris ${ }^{2}$, D. Pruss ${ }^{2}$, B. Roa ${ }^{1}$, R. Wenstrup ${ }^{1}$}

1. Myriad Genetic Laboratories, Inc.

2. Myriad Genetics, Inc.

Hypothesis/Purpose: Germline DNA sequencing analysis of $B R C A 1$ and $B R C A 2$ detects intronic or exonic variants which are predicted to result in abnormal mRNA splicing. While in silico splicing analysis may give some clues as to whether or not a particular variant may affect mRNA splicing, these analyses can potentially result in incorrect variant interpretation and inappropriate patient care. mRNA analysis may also lead to classification errors as splicing defects may be incomplete and abnormal mRNA products may not be detectable. We describe the algorithm used by our laboratory to determine pathogenicity of potential abnormal splicing variants. Methods: Sequencing analysis of the BRCA1 and BRCA2 genes identified exonic and intronic nucleotide changes predicted by in silicio splicing analysis to result in abnormal splicing. History weighting, co-segregation, and biochemical analyses were used to further assess variant pathogenicity. Results: Sequencing analysis of $>1$ million patients identified $\sim 1,500$ unique intronic variants in the BRCA1 and BRCA2 genes. In silico splice site analysis accurately identified some mutations as pathogenic splicing mutations. However, some 
variants predicted to result in abnormal splicing either have no effect on mRNA splicing or have a minimal effect that is not predicted to significantly increase cancer risk. In silico analysis also identified exonic variants which were predicted to result in abnormal mRNA splicing. Additional analyses confirmed the pathogenicity of some of these variants, but provided significant evidence that other variants are most likely benign. Conclusions: In silico splicing analysis may indicate that particular variants negatively affect mRNA splicing. However, many of these predictions are either inaccurate or splicing effects are small and do not result in significant increases in cancer risk. Therefore, in silico predictors should be used with caution and results rigorously verified by other independent methods before being used in the clinical setting.

\title{
The Impact of Testing Minors for Alpha-1 Antitrypsin Deficiency from the Perspective of Adults Tested as Children
}

\author{
A. Brown ${ }^{1}$, R. Stewart ${ }^{2}$, T. McConnell ${ }^{1}$, S. Wienke ${ }^{3}$ \\ 1. University of North Carolina at Greensboro \\ 2. Genetic Counseling Program, University of North Carolina at Greensboro \\ 3. The Medical University of South Carolina
}

Background: Alpha-1 antitrypsin deficiency (AATD) is one of the most common metabolic disorders, predisposing individuals to emphysema as adults and liver disease throughout the lifetime. Previous studies have not adequately measured the impact that testing minors for this condition has had on these individuals' health behaviors or psychosocial concerns. The goal of this research project was to assess the possible impacts of testing children for this condition and inform the discussion concerning the potential addition of AATD to newborn screening. Methods: Participants were recruited from two research registries and were asked to complete a single anonymous survey. Participants were eligible for this study if they were currently 18 years of age or older, had genetic testing for AATD prior to age 18, and were English proficient. Survey questions were developed based on review of the literature and self-esteem was assessed using the Rosenberg Self-Esteem Scale. Results: A total of 28 eligible responses were received. Most respondents reported having severe (12 of 28) or moderate deficiency (12 of 28). Experience with AATD related illness in the family influenced the likelihood that testing results would affect career choice. Those who had their results disclosed by a healthcare provider had lower self-esteem than those who had their results disclosed by their parents. All respondents agreed or neither agreed nor disagreed with the statement that AATD should be added to newborn screening tests. Eleven respondents suggested a better time in life when testing should occur. Discussion: More positive impacts of testing minors for AATD were reported than negative impacts. Many respondents reported that they were glad they had testing, would pursue testing in their children, and were interested in newborn screening for their families. There was not universal agreement among this population that AATD should be added to newborn screening tests for the general population. Implications for practice include improved education about smoking risks for minors tested for AATD.

\author{
Motivations for Presymptomatic Genetic Testing in C9orf72 Related Frontotemporal Degeneration and Amyotrophic Lateral Sclerosis Families \\ C. Chambers ${ }^{1}$, E. McCarty Wood ${ }^{2}$, K. Valverde ${ }^{3}$ \\ 1. University of Virginia \\ 2. University of Pennsylvania \\ 3. Arcadia University
}

Frontotemporal degeneration (FTD) and amyotrophic lateral sclerosis (ALS) are adult onset neurodegenerative diseases; the former being a form of dementia primarily affecting cognition, personality, and language, and the latter is a severe motor neuron disease. An autosomal dominant occurrence of FTD and/or ALS has been recently attributed to the C9orf72 mutation and presymptomatic testing is now available for those with a known familial mutation. Testing motivations in other adult-onset diseases have been documented but there is no current research exploring the reasons individuals would seek presymptomatic testing for the C9orf 72 mutation. This qualitative research study investigated presymptomatic testing motivations and attitudes among at-risk individuals. Eligible participants were asymptomatic adults ( $>18$ years old) that had a first degree relative with an identified C9orf 72 mutation. As part of their participation in a longitudinal clinical study, participants completed surveys that assessed current beliefs about their C9orf 72 risk, knowledge about the C9orf 72 mutation and associated diseases, and genetic testing motivations. Data analysis of completed surveys from twelve at-risk individuals was completed using descriptive statistics. Results suggest that individuals at-risk for FTD and/or ALS pursue presymptomatic testing to provide information for family members, to obtain relief from uncertainty, and to plan for the future. Motivations not to pursue genetic testing included no current cure, a preoccupation with the onset of symptoms, and concerns of genetic discrimination. All participants knew the C9orf72 gene can cause both FTD and ALS, but there were differing degrees of perceived risk for FTD compared to ALS. This study provides initial insight into not only genetic testing motivations but also the educational needs of individuals at-risk for C9orf72-related FTD and ALS. Such insight will enable genetic counselors to provide information and support that target the specific needs and concerns for individuals seeking presymptomatic genetic counseling.

Products of Conception Testing by SNP Microarray Analysis on Monozygotic Twin Gestations Reveals Discrepant Chromosome Results and the Scope of Aneuploidy in Mosaic Pregnancies

\section{Clark ${ }^{1}$, B. Pettersen ${ }^{1}$, M. Maisenbacher ${ }^{1}$, S. Sigurjonsson ${ }^{1}$, K. Paik ${ }^{1}$, M. Young ${ }^{1}$,}

1. Natera, Inc.

Objective: Report on five twin gestation products of conception (POC) cases where single nucleotide polymorphism (SNP) microarray and a bioinformatics technique confirmed monozygotic twins with discrepant aneuploidies. This method offers the unique ability to check SNP concordance to define twins as either monozygotic or dizygotic, identify uniparental disomy (UPD), rule out maternal cell contamination (MCC), and determine parental origin of abnormalities, none of which can be identified by karyotyping or array comparative genomic hybridization (CGH). Methods: Retrospective review of POC cases analyzed by a single reference lab. Maternal blood and POC samples were received. Two dissections were performed for twin gestation samples. Genotyping was performed using Illumina CytoSNP-12b microarray and bioinformatics. If either sample returned an MCC result, or the samples showed identical results, a third dissection was performed to identify a separate fetal result. Results: Five twin gestation cases were identified that showed identical SNP patterns on all chromosomes except one. Case 1 showed a normal male in one sample and trisomy 9 in a second. Cases 2 and 3 showed mosaic trisomy of chromosomes 2 and 9, respectively, in one sample and full trisomy of the same chromosome in a second. Case 4 showed full trisomy 16 of maternal origin in two samples with one also showing mosaicism of chromosome 7 of paternal origin. Case 5 showed trisomy 22 in one sample and maternal UPD 22 in the second. Conclusion: SNP microarray with bioinformatics provides a unique method to detect aneuploidies as well as zygosity and UPD in multiple gestation losses. Though it cannot be determined if the discrepant aneuploid results between two otherwise 
identical samples represents mosaicism within one fetus or represents two otherwise identical twins, trisomy rescue is a likely explanation. Without using maternal genotype, twin gestations may be incorrectly classified as non-identical and/or non-mosaic on the basis of discrepant chromosome results and the true scope of the chromosome abnormality may go unrecognized.

\section{Exploring Genetic Counselors' Perspectives on the Clinical and Personal Utility of Genetic Testing}

\section{M. Coon ${ }^{1}$, P. J. Hulick², M. E. Smith ${ }^{3}$, S. A. Aufox ${ }^{3}$, C. A. Wicklund ${ }^{3}$}

1. Northwestern University

2. NorthShore University HealthSystem

3. Center for Genetic Medicine, Northwestern University

Introduction: Clinical utility and personal utility are important concepts in evaluating genetic tests. However, there is no consensus on how these concepts are defined or related. We surveyed genetic counselors on how they categorize the utility of genetic tests to better understand what these concepts represent. Methods: This was a cross-sectional study to explore the concepts of personal and clinical utility as they relate to genetic testing. An online survey developed for this study asked respondents to characterize the utility of a genetic test in 25 different clinical scenarios and determine if the test warranted insurance coverage. Email invitations were sent on December 4, 2013 to full-members of the National Society of Genetic Counselors (NSGC) and on December 12, 2013 to Australasian Society of Genetic Counsellors (ASGC). An NSGC reminder email was sent 2 weeks later, and the survey closed on January 8, 2014. Descriptive statistics were used to report response frequencies and chi-square analysis or two-sided Fisher's exact tests for comparisons. Results: 381 genetic counselors participated. Response rate was $15.1 \%$ of NSGC and $5.7 \%$ of ASGC members. Respondents were representative of NSGC members in terms of age, gender, and primary specialty area, and demographics did not significantly influence utility characterization. Respondents were more likely to say a genetic test had both clinical and personal utility, more likely to think a test warranted insurance coverage if it had a higher level of clinical utility, and more likely to categorize a test as having clinical utility if a patient was at increased risk for a condition, the condition was preventable or treatable, or the results affected medical management. Conclusions: The results of this survey indicate there is no apparent consensus among genetic counselors about how to define clinical and personal utility; however, genetic counselors readily see the personal utility of genetic tests performed in a clinical context but feel insurance should cover those tests with a higher clinical utility.

\section{Hereditary Pancreatic Cancer Multi-Gene Test: Preliminary Results}

\section{E. Dalton ${ }^{1}$, J. Dolinsky ${ }^{1}$, H. Laduca ${ }^{1}$}

\section{Ambry Genetics}

Pancreatic cancer (PC) is recognized as a component tumor in many well-described hereditary cancer syndromes. Despite this, there are limited guidelines for ordering genetic testing for sporadic and familial PC. Through examining the clinical histories of individuals found to have a mutation on a hereditary PC multi-gene test, we explore how the increased availability of multi-gene testing could better define criteria for hereditary PC testing. DNA from 156 individuals with a personal and/or family history of PC was analyzed with PancNextTM, a multi-gene test including sequencing and deletion/ duplication analyses of the following genes: APC, ATM, BRCA1, BRCA2, CDKN2A, MLH1, MSH2, MSH6, PALB2, PMS2, STK11, and TP53; and deletion/duplication analysis of $E P C A M$. Clinical information was submitted by ordering healthcare providers. 18 mutations were identified among 16 participants (10.3\%), including 6 ATM, 2 MSH6, 4 BRCA, 3 APC (I1307K), 2 CDKN2A, and 1 PALB2 mutation. Neither of the MSH6 mutation carriers met Amsterdam II criteria for Lynch syndrome, and one of the BRCA mutation carriers did not meet National Comprehensive Cancer Network testing criteria for hereditary breast and ovarian cancer syndrome. Of individuals with mutations, $75 \%(n=12)$ had PC, diagnosed at a mean age of 54 years, compared to mutation-negative individuals, where $43 \%$ had PC, diagnosed at a mean age of 61 years. Seventy-five percent of patients with pathogenic mutations $(n=12)$ had a family history of PC, averaging 2 affected relatives. Similar to other cancers, a genetic predisposition to PC will be identified in about $10 \%$ of individuals undergoing genetic testing. Interestingly, $33 \%$ of the mutations identified were in the ATM gene, supporting findings from previous studies on the correlation of ATM mutations with hereditary PC. Additionally, the mean age of diagnosis in individuals with a mutation was younger than in mutation-negative individuals, suggesting that a younger age at diagnosis may be correlated with hereditary predisposition.

\section{Expanded Carrier Screening in the Ashkenazi Jewish Population}

\section{Elkhoury ${ }^{1}$, L. Shi ${ }^{1}$, J. Zhang ${ }^{1}$, G. Diaz ${ }^{1}$, L. Edelmann ${ }^{1}$, R. Kornreich ${ }^{1}$}

\section{Icahn School of Medicine at Mount Sinai}

American College of Medical Genetics and Genomics (ACMG) guidelines recommend that individuals with Ashkenazi Jewish (AJ) ancestry be offered prenatal or preconceptional carrier testing for 8 highly prevalent diseases (and several laboratories provide testing for an additional 9). Although larger expanded pan-ethnic panels that include some AJ specific mutations are available, they are not focused on disorders affecting AJ individuals. We sought to establish a more comprehensive carrier screening panel than is currently available for this population based on 1) additional disease-associated founder mutations or 2) recurrent pathogenic variants that have been reported in AJ individuals. The study included early onset or progressive, severe diseases. Anonymized DNAs from individuals self-reported as $100 \%$ AJ were subjected to targeted genotyping. Sixty seven mutations from 18 genes representing 18 autosomal recessive disorders were selected for the final panel based on results from approximately 2,000 samples. This expanded panel includes 5 diseases with carrier frequencies higher than $1 \%$ (Smith-Lemli-Opitz syndrome 1/36, carnitine palmitoyltransferase II deficiency $1 / 51$, congenital amegakaryocytic thrombocytopenia $1 / 55$, congenital disorder of glycosylation 1a $1 / 57$, Wilson disease $1 / 70$ ); the remaining diseases had the following carrier frequencies (autosomal recessive polycystic kidney disease 1/107; BBS2-related Bardet-Biedl syndrome 1/107; retinitis pigmentosa $591 / 118$; tyrosinemia type $111 / 150$; galactosemia 1/172; PEX2-related Zellweger syndrome 1/172; abetalipoproteinemia 1/180; Alport syndrome 1/188; dyskeratosis congenita, autosomal recessive 1/203; Ehlers-Danlos VIIC 1/248; 3phosphoglycerate dehydrogenase deficiency 1/280; multiple sulphatase deficiency 1/320; arthrogryposis, mental retardation and seizures 1/373). Our AJ carrier screening panel now routinely includes 38 disorders which results in 1 in 2 AJ individuals being identified as a 
carrier for at least 1 of these disorders. Results from this ongoing screening will be presented including an individual identified as a carrier for 5 disorders.

\title{
200 Days of Multi-Gene Testing: The Clinical Experience
}

\author{
A. Forman ${ }^{1}$, K. Rainey ${ }^{1}$, S. Montgomery ${ }^{1}$, C. Rybak ${ }^{2}$, A. Masny ${ }^{1}$, E. Obeid ${ }^{1}$, M. Daly ${ }^{1}$, M. Hall ${ }^{1}$ \\ 1. Fox Chase Cancer Center \\ 2. City of Hope
}

Background: Advances in cancer genetic testing technology to include next generation sequencing (NGS) along with removal of patent barriers have allowed expansion beyond syndrome-specific testing to multi-gene NGS panels. As these panels become more widely implemented, we describe the early clinical experience of a National Cancer Institute designated comprehensive cancer center in offering NGS panels to all eligible patients. Methods: Beginning 9/2013, patients were offered syndrome specific testing or an NGS panel as appropriate. Initially, most genetic testing naive patients were offered a 25 gene comprehensive cancer panel as part of an early access program. Panel test options became more varied over time and include non-breast cancer panels. All patients met testing criteria established by the National Comprehensive Cancer Network or were approved for testing by a genetic counselor. Results: Genetic counseling was performed for 375 patients. Germline testing was ordered for 271, of whom 165 (60.8\%) chose some type of NGS panel. BRCA1/2 testing alone was pursued by 43 (15.9\%) patients; $32.5 \%$ were treatment decisions and $50 \%$ declined a panel due to limitations, e.g. rate of variants of uncertain significance (VUS) and potential for unclear medical management guidelines. We received results for 151 panels and mutations were identified in $11.2 \%$. These 17 positive results include $5 \mathrm{MYH}$ heterozygotes and $2 \mathrm{APC}$ I1307K variants, which provide unclear cancer risk influence. At least one VUS was found in $35.1 \%$ of patients compared to $7.5 \%$ of $B R C A 1 / 2$ results $(p=0.0007)$. Of note, non-white patients had a $53.6 \%$ risk of finding a VUS compared to $31.4 \%$ in whites $(p=0.0272)$. Discussion: NGS panels can yield results not found in traditional testing, but also raise questions regarding findings with unclear cancer risks. A significantly increased risk for VUS results, particularly in non-white patients, may complicate education and patient understanding. Due to limitations in NGS panels, a subset of patients will choose gene-specific testing instead of a panel.

“The Sooner the Better:" Genetic Testing following Ovarian Cancer Diagnosis

E. Fox ${ }^{1}$, J. McCuaig ${ }^{2}$, R. Demsky², C. Shuman ${ }^{3}$, D. Chitayat ${ }^{3}$, S. Ferguson ${ }^{4}$, S. Armel ${ }^{2}$

1. University of Toronto

2. Familial Breast and Ovarian Cancer Clinic, Princess Margaret Hospital

3. Division of Clinical and Metabolic Genetics, Hospital for Sick Children

4. Princess Margaret Hospital

Introduction: The discovery of the $B R C A 1$ and $B R C A 2$ genes has allowed for better risk assessment and screening programs, but despite this advance, many mutation carriers are not identified until after they develop cancer. As treatment based on genetic testing becomes a reality it is valuable to assess the attitudes and preferences of women newly diagnosed with ovarian cancer regarding genetic testing. The objective of this study was to determine when women with a diagnosis of high grade serous ovarian cancer would prefer to undergo genetic testing and which factors influence this preference. Methods: We identified women over 18 years of age with a known diagnosis of high grade serous ovarian cancer diagnosed between October 2010-2013 via the Princess Margaret Cancer Center Registry. Participants completed a questionnaire by mail or in clinic including demographic information, cancer history, and preferences and attitudes towards genetic testing. Responses were analyzed using PASS and analysis included multiple linear regression for factors associated with timing preferences. Results: We received questionnaires from 97 of the 315 women identified ( $30 \%$ ). The mean age of ovarian cancer diagnosis was 59 years of age. The vast majority of the women participating in this study were offered genetic testing $(89 \%)$ and opted to pursue it $(88 \%)$. In this population, testing was most frequently offered at diagnosis $(39 \%)$ or during treatment $(18.5 \%)$. Preliminary data indicate that women with high grade serous ovarian cancer feel genetic testing should be offered at time of diagnosis $(50.5 \%)$. Reasons given for preferring genetic testing at initial diagnosis were to help understand why cancer developed, to inform treatment, and for information for family members. Conclusion: These results demonstrate that women with high grade serous ovarian cancer acknowledge the personal and clinical utility of genetic testing and support test implementation at the time of cancer diagnosis.

\section{Male Rett Syndrome Resulting from an MECP2 Missense Variant}

J. Hartley ${ }^{1}$, M. Salman ${ }^{2}$, A. Mhanni ${ }^{1}$

1. Genetics and Metabolism, Health Sciences Centre

2. Pediatric Neurology, Health Sciences Centre

Introduction: Rett syndrome is an X-linked disorder caused by mutations in the MECP2 gene. It was initially described in females and was thought to be lethal in males. MECP2 mutations are now reported in 1.3-1.7\% of males with developmental delay. The presentation is variable as some males have classic features whereas others can present with an encephalopathy, psychiatric disturbances or isolated intellectual disability. Case: We report a male who presented at 15 months with a history of developmental delay, truncal ataxia and upper limb tremor with no history of developmental regression. The pregnancy and family histories were unremarkable. The neonatal history was remarkable for apneas. Tonic-clonic seizures were noted at 19 months, which were controlled on clobazam. He is non-dysmorphic and normocephalic. Brain MRI is unremarkable. Extensive metabolic studies in blood, CSF, urine and muscle were normal. Conventional karyotype, chromosome breakage studies, array CGH, and 15q11.2-q13 DNA methylation studies were normal. Sequencing of the MECP2 gene revealed a de novo previously unclassified variant, c. $674 \mathrm{C}>\mathrm{T}$ ( $\mathrm{p} . \mathrm{R} 225 \mathrm{~L}$ ). At 10 years, he is non-ambulatory, averbal, ataxic, and has behavioral issues and a movement disorder. Discussion: This $\mathrm{C}>\mathrm{T}$ substitution in exon 3 leads to the substitution of non-polar 
amino acids, proline to leucine, in the transcriptional repression domain. In silico analyses provided conflicting results, and therefore the substitution was reported as an unclassified variant. This variant has not been reported in the general population, but has been previously reported as a de novo alteration in a male presenting with mental retardation, spastic paraplegia, dystonia, apraxia, and scoliosis. A missense mutation in the same position (c.674C $>\mathrm{G}$ ) was also identified in several affected females. Our patient appears to be the second reported occurrence of this sequence variant in a male presenting with symptoms consistent with a phenotype of an MECP2 related disorder.

\section{Novel Variant Identified in $C Y P 7 B 1$ Using Next-Generation Sequencing}

C. Hartshorne' ${ }^{1}$, J. Kobori ${ }^{2}$, C. Vendola ${ }^{2}$, M. Anderson ${ }^{1}$, M. Powers ${ }^{1}$

\section{InVitae Corp}

2. Kaiser Permanente

Hereditary spastic paraplegias (HSP) are a rare group of disorders with an estimated prevalence of 1.3-9.6/100,000 births. At least 41 genes have been associated with HSP and inheritance can be autosomal dominant, recessive, X-linked or mitochondrial. Symptoms can begin in childhood and be non-progressive or after childhood and be progressive. Spastic paraplegia type 5A (SPG5A) is an autosomal recessive form that can be uncomplicated or include axonal neuropathy, distal or generalized muscle atrophy and white matter anomalies on MRI. The only known cause of SPG5A is the CYP7B1 gene. We describe a 12 year-old girl, born from non-consanguineous parents, referred to genetics for acquired progressive spastic diplegia. Pregnancy and family history were non-contributory. She had normal gross and fine motor development until roughly 8 years old; she then presented with gait abnormalities with increased falling and clumsiness. She had evidence of spasticity with bilateral ankle clonus and possible sensory deficits. MRI, biochemical screening, and heavy metal titers were normal. Genetic testing for spinocerebellar ataxia, dentatorubral-pallidoluysian atrophy (DRPLA) and array CGH revealed no abnormalities. Next-generation sequencing of 17 genes associated with HSP revealed two variants in CYP7B1: a known pathogenic missense change leading to a premature stop codon (c.334 C>T p.R112X) and a novel missense change of uncertain significance (c.788 T $>$ G p.V263G). Prediction algorithms suggested the novel missense variant to be likely disruptive, however no confirmatory functional studies were identified. Parental studies were performed and confirmed the two variants to be in trans, classification of the novel missense variant was changed to likely pathogenic. This case presents a new diagnostic variant for this rare disorder. Due to the various modes of inheritance of HSP, as well as overlapping symptoms with non-hereditary disorders, it is important to determine the underlying cause of HSP cases to provide the most accurate reproductive risks for individuals affected with this disorder and their families.

Factors Affecting Decision Making of Social Workers about Preadoption Genetic Testing

D. Julian' ${ }^{1}$, B. Yashar ${ }^{1}$, W. Uhlmann ${ }^{2}$, K. Freeark-Zucker ${ }^{1}$, J. Roesch ${ }^{3}$

1. University of Michigan

2. University of Michigan Medical Center

3. Hands Across the Water

Best interest of the child, a central tenet in adoption, is realized by striving to place the child with the most suitable family for their needs. While the information provided by preadoption genetic testing (a genetic testing request by prospective adoptive parents and/or the adoption agency on the child prior to adoption) is postulated to help social workers find the best placement, the impact on the decision-making process is unknown. A clinical scenario centered on a child with a family history of von Hippel Lindau disease was used to evaluate this process. A 29 question survey was created by a multidisciplinary committee and distributed through 6 midwestern National Association of Social Work chapters. Information collected included individual and adoption agency demographics, genetics education, and facets of the adoption process. Response frequencies were calculated using SPSS. In our sample of 129 social workers, the majority $(68 \%)$ of participants answered 'yes' to ordering genetic testing. "Genetic testing would affect care of the adoptee" was the most important factor in this decision (26\%), with the second most frequently ranked factor being "prospective adoptive parent's right to know the genetic information" (19\%). When results were separated by respondent test decision, those who selected 'yes' to ordering genetic testing most frequently ranked "genetic testing would affect care of the adoptee" (20\%) first, while those who selected 'no' ranked "genetic testing should be determined by the adoptee" (12\%) first. The adoptee's autonomy was also valued highly in this scenario. Our results indicate that adoptee genetic information is weighed by social workers in supporting the best interest of the child and the prospective adoptive parent's right to know. These potentially conflicting priorities highlight the tension between supporting a child's medical needs and the desire to preserve an open future. This study identifies an opportunity for genetic counselors to collaborate with social workers about decision-making in nuanced genetic testing situations.

Going Beyond the Guidelines: A Call for Expanded Jewish Carrier Screening Based on an Analysis of 500 Clinical Samples Screened via an Expanded Carrier Screening Platform

G. Kellogg ${ }^{1}$, N. Kumar ${ }^{1}$, S. Rodriguez ${ }^{1}$, A. Bisignano ${ }^{1}$, S. Munne ${ }^{2}$, J. Fischer ${ }^{2}$

1. Recombine

2. Reprogenetics, LLC

Introduction: Current guidelines recommend offering carrier screening for specific diseases to Ashkenazi Jewish individuals (American College of Obstetricians and Gynecologists, 4 diseases; American College of Medical Genetics and Genomics (ACMG), 9 diseases). We found that carrier rates for 
diseases that are not currently included in guidelines are significant, and higher than expected based on the literature. Further, we found that these guidelines do not incorporate diseases common to individuals of non-Ashkenazi Jewish heritage, e.g. Sephardic Jews, which is important given the increasing admixture of these populations. Materials and Methods: The Illumina Infinium HD Custom Genotyping platform was used to identify 1,679 mutations associated with 213 recessive genetic diseases. The analysis includes data obtained from 500 clinical referrals. Documented informed consent to utilize data in a de-identified manner was obtained. Carrier rates for each disease were calculated for Jewish individuals and were compared with the literature. Detection rates for these conditions were determined based on the literature. Results: We found high carrier rates in Jewish individuals for diseases for which Jewish population screening is not currently recommended, including: Congenital Disorder of Glycosylation: Type 1A (1/42), Smith-Lemli-Opitz syndrome (1/45), Glycogen Storage Disease: Type II (1/50), Glycogen Storage Disease: Type 1A (1/71), Familial Hyperinsulinism (1/100), and Amegakaryocytic Thrombocytopenia (1/100). Additionally, the carrier rate for Walker-Warburg syndrome (1/50) is significantly higher than previously reported (1/150). Conclusions: ACMG guidelines suggest the addition of diseases to Jewish carrier screening panels if the disease has a carrier frequency of $>=1 \%$ or a detection rate $>90 \%$ in Jewish individuals. We have identified more than 50 diseases that meet these criteria in Jewish individuals, as well as several diseases (Walker-Warburg syndrome) whose carrier frequency has been previously underestimated in the literature, and may be appropriate for Jewish population screening.

A Single Institution's Experience with the myRisk Panel: Exploring the Rates of Incidental Findings and Variants of Uncertain Significance

B. Leach ${ }^{1}$, J. Moline ${ }^{1}$, J. Polk ${ }^{2}$, R. Noss ${ }^{3}$, J. Mester ${ }^{1}$, M. Nardini ${ }^{1}$, R. Cook ${ }^{3}$, C. Eng $^{3}$

1. Cleveland Clinic Foundation

2. Hillcrest Hospital

3. Cleveland Clinic

The decreasing cost and increasing access of next generation sequencing has led to multiple gene panels tailored for hereditary cancer syndromes. While the technology has been shown to be valid, there are limited data on clinical outcomes, utility, and variant detection rates. We describe our experience at a single academic institution with Myriad Genetics Laboratory's myRisk Panel. Patients underwent formal genetic risk assessments at Cleveland Clinic, and myRisk was ordered based on clinical suspicion of Hereditary Breast Ovarian Cancer and/or Lynch syndrome. Gender, ancestry, age at testing, history indicated on the test requisition, and genetic test results were queried from Myriad's database. Test results were correlated with clinical risk assessments. Descriptive statistics were utilized. Between 9/4/2013 and 4/17/2014, 309 myRisk assays were performed. Overall, 31 deleterious mutations (20 in high risk genes, 11 in moderate risk genes) were identified in 29 patients (9\% positive rate). Three patients had 2 deleterious mutations ( $P T E N / N B N, B R C A 2 / P M S 2$, CHEK2/NBN). Six patients $(2 \%)$ had mutations in high-risk genes of low clinical suspicion or incidental findings that significantly impacted management (APC, BRCA2, CDH1, 3 in $P M S 2)$. In addition, one patient had $A P C$ I1307K, and five patients had monoallelic $M U T Y H$ mutations. Overall, 177 variants of uncertain significance were detected in 128 patients $(41.4 \%)$. The majority of variants were in moderate risk genes $(60.4 \%)$. The variant rate was lower in those of western/northern European ancestry $(35 \%)$ than African (52.6\%). Most patients had only one variant $(67.2 \%)$ while one patient had four. These data help to guide counseling about myRisk, in particular establishing the frequency of incidental findings and highlighting the variant detection rate. Additional data are needed to determine if incidental findings and variant detection rates are comparable to other next generation sequencing panels.

\section{A Case of Prenatal Detection of Duplication (21) (q22.2q22.13): Use of Multiple Testing Methodologies to Clarify Apparently Discordant Results}

K. Levandoski ${ }^{1}$, K. Marchand ${ }^{1}$, K. Patek $^{2}$

1. Beth Israel Deaconess Medical Center

2. Quest Diagnostics

In pregnancies with the finding of an increased nuchal translucency or detection of aneuploidy marker, noninvasive prenatal testing (NIPT) is often offered prior to diagnostic testing. The scope of NIPT, however, is limited and cannot detect all possible chromosomal etiologies. When diagnostic testing is pursued, standard karyotype analysis is limited in its ability to detect small copy number changes. The utility of chromosome microarray to detect these small changes has been previously reported. We report on a 30-year-old G3P1 woman who presented to our clinic at 16 weeks 5 days for fetal survey. Outside records indicated increased nuchal translucency $(3.6 \mathrm{~mm}$ ) and low risk NIPT results (MaterniT21 PlusTM). Nuchal fold measuring $7.2 \mathrm{~mm}$ and echogenic intracardiac focus were noted at the patient's survey. Amniocentesis was performed. FISH results indicated a male fetus with trisomy 21. Preliminary karyotype showed disomy 21. Follow-up metaphase FISH showed 3 signals for 21 (probe at q22.13-q22.2) with two signals on one chromosome 21, raising suspicion for a tandem duplication. Microarray analysis was completed and revealed a $3.5 \mathrm{Mb}$ duplication on chromosome 21: 46,XY,dup(21) (q22.13q22.2). This duplication was expected to be associated with physical and/or intellectual features similar to those seen with a diagnosis of trisomy 21 as it involved a portion of the Down syndrome critical region. Although discordant results seemed to be present in this case, both NIPT and initial FISH results were correct within the scope of their testing abilities. This case highlights the importance of pre- and post-test counseling for patients undergoing NIPT for ultrasound findings, the utility of chromosome microarray to detect small copy number changes otherwise undetectable by standard karyotype, and the importance of open communication between the ordering provider and the lab completing the testing to provide the best care for our patients. 


\title{
A Comparison of Traditional and Panel Testing for Hereditary Breast and Ovarian Cancer in over 900 Patients
}

\author{
S. Lincoln ${ }^{1}$, A. Kurian², S. Kristen ${ }^{3}$, A. Desmond ${ }^{3}$, Y. Kobayashi' ${ }^{1}$, M. Anderson ${ }^{1}$, M. Rabideau' ${ }^{1}$, M. Mills ${ }^{2}$, M. Gabree ${ }^{3}$, J. Ford ${ }^{2}$, L. Ellisen ${ }^{3}$ \\ 1. Invitae \\ 2. Stanford University \\ 3. Massachusetts General Hospital
}

Broad multi-gene panels for hereditary cancer are now entering clinical use. To help develop the most appropriate genetic counseling practices for panels it is important to have careful measurements of their yield and performance by comparison with traditional genetic tests. Recently we described clinical results from panel testing of 198 patients which we expand on here. We have collected over 900 patients to date from two clinical centers, all of whom had been referred for BRCA1/2 testing under National Comprehensive Cancer Network guidelines. 400 of these patients were prospectively recruited over the past year in order to represent current clinical experience, while the remainder were retrospective cases. All received testing with a 29-gene panel, including BRCA1/ 2, as well as traditional testing by another lab for $B R C A 1 / 2$ alone. For $B R C A 1 / 2$, we see $100 \%$ concordance between the next generation sequencing (NGS) panel and the traditional (Sanger) tests. While certain classes of events are known to be challenging for NGS (e.g., large sequence indels and small copy-number deletions/duplications) we find that these challenges can be overcome. We also find $99.8 \%$ agreement on pathogenicity assessments between the two labs. In $8 \%$ of $B R C A 1 / 2$ negative patients we see positive findings in other genes including TP53, CDH1, MLH1, MSH2, MSH6, PMS2, CHEK2, BRIP1, PALB2, and RAD51C. Counseling these patients often includes a level of uncertainty, as the gene may confer a modest increased risk, and in some cases the spectrum of cancers in the family does not fit the spectrum commonly associated with mutations in that gene. About half of our non-BRCA1/2 positives are heterozygous carriers of recessive cancer risk genes $A T M, N B N$, and $M U T Y H$. In addition to the uncertain risks, counseling must address the different reproductive and family implications compared to the dominant gene findings. We conclude that gene panels can provide reliable data and deliver important findings missed by traditional testing, presenting many new implications for genetic counseling.

\section{One Disease, Multiple Solutions: A Family Case Study of Preconception and Prenatal Testing for Duchenne Muscular Dystrophy and Exploration of Reproductive Testing Options}

\section{Maisenbacher ${ }^{1}$, N. Wemmer ${ }^{1}$, B. Pettersen ${ }^{1}$}

\section{Natera, Inc.}

Clinical Findings: Duchenne muscular dystrophy (DMD), a lethal genetic disorder characterized by progressive muscle degeneration and weakness, is caused by a deletion, insertion, or point mutation in the DMD gene located on the X chromosome. DMD has an X-linked recessive inheritance pattern, thus carrier mothers have a $25 \%$ risk with each pregnancy to have an affected son. Evaluation: Patient and partner were referred by an in vitro fertilization (IVF) center to a reference lab for single gene preimplantation genetic diagnosis (PGD) for DMD with concurrent 24-chromosome aneuploidy screening using single nucleotide polymorphism (SNP) microarray with bioinformatics (Parental SupportTM). Patient was a 31-year-old confirmed carrier of a DMD deletion, spanning exons 45-49, with a positive maternal family history of DMD. Results: Three embryo samples from the first IVF cycle showed unaffected male or carrier female and euploid results. The remaining 7 embryo samples had results showing either affected with DMD or aneuploidy, or did not return results. One carrier female embryo was transferred resulting in a healthy baby girl. The other two unaffected and euploid embryos were of poor quality and not suitable for transfer or cryopreservation. Prior to undergoing a planned second IVF cycle with PGD, the patient became pregnant naturally. A maternal blood sample was sent to the same reference lab for noninvasive prenatal testing (NIPT) at 9 week 2 days gestation. NIPT results were low risk for trisomy 21, 18, 13, Monosomy X and Triploidy. Gender was female. Follow-up ultrasounds have been normal. The couple declined invasive testing and are due mid-July.. Discussion: Couples at risk for X-linked single gene disorders may consider various types of reproductive testing options, including IVF with PGD, invasive procedures, and NIPT. Only IVF with PGD provides information prior to embryo implantation. The introduction of NIPT for gender adds a new early and noninvasive screening test for X-linked conditions that may help guide follow-up prenatal care recommendations.

\section{ClinVar: The Respository for Interpretations of Clinically-Relevant Variations}

\section{A. Malheiro ${ }^{1}$, B. Kattman ${ }^{1}$, W. Rubinstein ${ }^{1}$, M. Landrum ${ }^{1}$, G. Riley ${ }^{1}$, J. Lee $^{1}$, D. Maglott ${ }^{1}$ \\ 1. National Center for Biotechnology InformationI / U.S. National Library of Medicine / National Institutes of Health}

ClinVar (http://www.ncbi.nlm.nih.gov/clinvar/) is a new database developed to facilitate the evaluation of the relationship and clinical significance of variants to phenotypes. ClinVar aggregates and archives these relationships and submitted interpretations with supporting evidence, by receiving data from multiple groups such as laboratories to determine if there is a consensus about the interpretation, and by making summary data freely available. ClinVar's primary goal is to provide access to a comprehensive dataset of clinically relevant variants observed in humans, their phenotypic relationships, current interpretations, and supporting evidence. ClinVar, which launched its public webpage in April of 2013, currently has over 110,000 submissions from more than 125 submitters representing over 99,000 variants in more than 18,000 genes. Submitters include testing laboratories, the Breast Cancer Information Core (BIC) and other locus- 
specific databases, researchers, professional societies, as well as other resources such as OMIM ${ }^{\circledR}$ and GeneReviews ${ }^{\circledR}$. ClinVar participates in the ClinGen collaboration, providing information for expert panel curation, and archiving the results of review. ClinVar also leverages the wealth of public genomic information by incorporating data from and links to reference sequences, dbSNP, dbVar, MedGen and GTR among others. ClinVar welcomes submissions from clinical and research laboratories, locus-specific databases, and curated publications. Submissions may include supporting evidence such as observations of the variant in affected or unaffected individuals, family studies, in vitro experiments and animal models. The integrated records also report data such as molecular consequence, standardized HGVS nomenclature, and allele frequencies from large-scale studies. Here we will introduce ClinVar from the clinical (user) and submitter perspectives, focusing on using ClinVar to locate information on the clinical significance of variants and how to submit data.

\section{Meckel-Gruber Syndrome in 6A Fetus Found to be Compound Heterozygous for Mutations in TMEM231 and Heterozygous for a Mutation in MKS6}

\section{K. Marchand ${ }^{1}$, J. Hecht ${ }^{1}$, C. McArdle ${ }^{1}$, V. Kimonis ${ }^{2}$, T. Takoudes ${ }^{3}$, T. Attie-Bitach ${ }^{4}$}

1. Beth Israel Deaconess Medical Center

2 University of California, Irvine

3. Boston Maternal Fetal Medicine

4. Hôpital Necker-Enfants Malades

Meckel-Gruber syndrome is a lethal multiple congenital abnormality syndrome within the ciliopathy family of disorders. We present a case of suspected fetal Meckel-Gruber syndrome (MKS), confirmed 8 years later through research testing. Ultrasound findings at 20 weeks gestation included large Dandy-Walker cyst, hydronephrosis, bilateral upper extremity polydactyly, and shortened forearms. Pathology evaluation of products of conception following termination showed additional findings of hypospadias, gonadal dysgenesis, accessory spleen, cleft epiglottis, upper and lower forelimb shortening, dysplastic cystic kidneys, and liver with myofibroblastic nodules with peripheral bile duct proliferation. Karyotype analysis showed normal male chromosomes, 46,XY. Clinical molecular genetic testing for MKS was not available, in 2006. Research testing was initiated (Paris, France) and recently identified the fetal tissue as being compound heterozygous for mutations in the TMEM231 gene. Interestingly, the fetus was also identified as being a heterozygote for a paternally-inherited mutation in the CC2D2A (MKS6) gene. All three mutations are predicted to be pathogenic. Homozygous TMEM231 mutations have previously been identified as causing classic MKS (encephalocele, polydactyly, and polycystic kidneys) in two consanguineous Arab families and compound heterozygous mutations have been found in individuals affected with Joubert syndrome. Our patient is the first report in the literature of TMEM231 compound heterozygosity causing a MKS phenotype and adds to the documentation of phenotypic variability (non-classic MKS in our patient). The presence of a concurrent pathogenic MKS6 mutation raises the question of whether phenotypic variability may be influenced by the presence of mutations in other MKS-related genes. After results disclosure this patient indicated that she had always questioned her potential role in causing the fetal abnormalities, highlighting the psychological importance of pursuing molecular diagnosis even when the clinical diagnosis appears clear.

\section{Understanding Patient Motivation and Factors that Influence Decisions on Prenatal Testing}

\section{Masso ${ }^{1}$, K. Paulyson Nunez ${ }^{2}$, R. Stewart ${ }^{3}$, D. Wells ${ }^{2}$}

1. University of North Carolina at Greensboro

2. Duke University Medical Center

3. Genetic Counseling Program, University of North Carolina at Greensboro

Introduction: Previous research has demonstrated the complexity of decision-making in a prenatal setting. The clinical introduction of noninvasive prenatal testing (NIPT) has further increased this complexity. Purpose: We sought to examine the decision-making process by surveying women to determine what testing/screening options are offered to our patient population; what options patients selected; what factors influence patients' decisions; how these differ among demographic groups; and what potential barriers disrupt decisionmaking vs. factors that facilitate the process. Methods: Participants were recruited by genetic counselors at Duke Medical Center from three clinical sites. Inclusion criteria were English-speaking patients 18 or older who received genetic counseling and were offered one or more prenatal testing options. Quantitative data was analyzed using the Wilcoxon rank sum test, Spearman's bivariate nonparametric correlation and chi-square test; qualitative data was analyzed to identify themes. Results: Of 110 women, NIPT was selected by $39.8 \%$, ultrasound only by $34.7 \%$, first trimester screening by $18.4 \%$, CVS by $1.0 \%$, and $6.1 \%$ were undecided. Safety/risk to the fetus, accuracy of the test, and ability to provide information early in pregnancy had the highest importance. Comparing women who chose first trimester screening vs. NIPT, there was a significant difference in ranking of cost/insurance coverage $(p=0.009)$. Factors that made decision-making easier included personal experiences, influence of external individuals, and specifics of the test. Concerns about stress or worry and cost of the testing made the process more difficult. Conclusions: Women consider many factors when deciding about prenatal testing. Aside from income level, most demographic factors did not predict testing option chosen. Cost/insurance coverage is an important influence and may affect whether women choose more accurate screening or not. Information is valuable to most participants, despite concerns of stress or worry and regardless of opinions towards pregnancy termination. 
Genetic Analysis of 113 Usher Syndrome Cases Using a Nine Gene Next Generation Sequencing Panel and a Targeted Array Comparative Genomic Hybridization

\author{
M. Meltzer ${ }^{1}$, B. Williams ${ }^{1}$, H. Elloumi ${ }^{1}$, D. Blain ${ }^{2}$, K. Goetz $^{3}$, M. Reeves ${ }^{2}$, A. Garafalo ${ }^{4}$, R. Parrish ${ }^{4}$, X. Wang ${ }^{2}$, S. Tumminia ${ }^{2}$, W. Zein ${ }^{2}$, N. \\ Smaoui ${ }^{1}$ \\ 1. GeneDx \\ 2. National Eye Institute, National Institutes of Health \\ 3. eyeGENE, National Eye Institute, National Institutes of Health \\ 4. Astrix Technology Group
}

Usher syndrome is an autosomal recessive disorder characterized by visual and hearing impairments and by extreme genetic and phenotypic heterogeneity. We have developed a 9 gene panel that includes sequencing of CDH23, GPR98, MYO7A, PCDH15, USH1C, USH1G, WHRN/DFNB31, USH2A, CLRN1 through next generation sequencing. Deletion and duplication testing of all nine genes through targeted array CGH analysis with exon-level resolution (ExonArrayDx) was performed in some cases when only one mutation or a variant of unknown significance was identified. We present our findings after 26 months of test utilization. Of the 113 cases referred with a clinical diagnosis of Usher syndrome, the molecular diagnosis was confirmed, i.e. two disease-causing mutations, or one disease-causing mutation and a variant likely disease causing (VLM) identified in the same gene, in $56 \%$ (63 cases); a single disease causing mutation was identified in $15 \%$ (17 cases); only variants of unknown significance (VUS) were present in $15 \%$ (17 cases). We have reported 208 mutations (91 unique), 56 VUS, and 16 VLM across nine genes. Only two mutations have been identified in CLRN1 to date. Variants were found in multiple genes in 28 of the 113 patients, with a mean of 2.4 variants/case. Of those 28,12 had two mutations in the same gene plus additional variants and four cases had one mutation in each of two or three genes. ExonArrayDx has been requested as follow-up testing for eight patients. As a result, copy number variants, including two partial deletions and two partial duplications in the USH2A gene, as well as two partial duplications in the GPR98 gene, were identified. Genotype-phenotype correlation analysis is in progress. In conclusion, our data strongly support that next generation Ssequencing combined with ExonArrayDx enable the lab to identify mutations and variants in a majority of patients with the clinical diagnosis of Usher syndrome.

The Impact of Fragile X Newborn Screening Results on Reproductive Choices and Surveillance for FMR1-Associated Disorders

P. Miranda ${ }^{1}$, L. J. Abrams ${ }^{2}$, J. Youngblom ${ }^{1}$, K. Basuta ${ }^{3}$, F. Tassone ${ }^{3}$

1. California State University Stanislaus

2. East Bay Genetic Counseling and Consultation

3. University of California, Davis, MIND Institute

The aim of the study was to explore the impact of fragile $\mathrm{X}(\mathrm{FX})$ premutation (PM) newborn screening (NBS) results on parents and family members' reproductive plans and surveillance for FMRI-associated disorders. A proposed benefit of detecting newborns with a PM through NBS is early intervention, considering the small risk of learning, behavioral, medical, and mental health issues. Identification of relatives with the PM may be beneficial for surveillance and treatment of FMRl-associated tremor ataxia syndrome (FXTAS) and primary ovarian insufficiency (FXPOI). However, it may cause worries for families, even though presentation of problems is uncommon and uncertain. Quantitative and qualitative data collection included a survey administered to family members of newborns with a PM, which was analyzed using descriptive statistics. All parent participants reported that they do not worry about their child meeting their developmental milestones; some expressed worry in the beginning. The majority of parents did not think it was likely that their children were at risk for FMR1-associated disorders. Individuals at-risk for having additional children with a PM or FX syndrome reported that they have not changed their reproductive plans; although some indicated consideration of alternate reproductive options. Two participants were identified with a PM and both were not concerned about developing FXTAS or POI. NBS does not seem to cause additional concerns for families of newborns with a PM, considering the small risks for FMR 1 -associated disorders in infancy or childhood and adult onset risks for PM carrier relatives.

\title{
Genotype Does Not Always Clarify Phenotype: Diagnosis Doesn't Stop with DNA
}

\author{
K. Morphy ${ }^{1}$, S. Zaghab ${ }^{2}$, M. M. Skinner ${ }^{2}$, C. Greene ${ }^{2}$ \\ 1. Allegheny General Hospital \\ 2. University of Maryland School of Medicine
}

Important reasons for DNA testing in a patient known or suspected to have a genetic disorder include clarifying prognosis and guiding management. We report 2 patients for whom genetic testing was performed in order to gain more information regarding expected phenotypic presentation; however, results lead to more uncertainty. Patient 1 presented at 17 years old with cardiac arrest, borderline prolonged QT and family history of syncope. Testing revealed a partial deletion of exons 11-12 in the SCN5A gene. Mutations in SCN5A have been associated with variable phenotypes, including Long QT Syndrome (LQTS), Brugada syndrome, and Dilated Cardiomyopathy. Large gene deletions, like the one in our patient, suggest a loss of function. Typically, loss of function mutations in SCN5A cause Brugada syndrome, whereas gain of function mutations cause LQTS. Our patient's clinical presentation wasn't consistent with Brugada syndrome. Therefore, results increased uncertainty with respect to her expected clinic course. Patient 2 is a 15 year old male with episodes of weakness and muscle cramps after exercise and family history of periodic paralysis (PP). Mother reports treatment with potassium-rich food (therapy for hypokalemic PP (HOKPP)) as well as high-carbohydrate intake (therapy for hyperkalemic PP (HYPP)). We attempted to clarify whether he had HYPP or HOKPP by analyzing a panel of PP genes. Patient has a Thr704Met 
mutation in the SCN4A gene. Mutations in this gene typically lead to HOKPP; however literature suggests this specific substitution leads to HYPP. Combination of family and patient history favors HYPP. However, uncertainty remains so trials of therapy with medications to target channel function will be undertaken. Use of DNA testing to understand etiology of disease and to predict prognosis is a valuable tool and continues to improve. Nevertheless, these two cases illustrate that determination of genotype via genetic testing does not always provide a clear expectation for phenotype or guidance for management

\title{
Oocyte Donor Genetic Screening Practices
}

\author{
E. Moyle ${ }^{1}$, A. Mathiesen ${ }^{2}$, E. Johnstone ${ }^{3}$, R. Hulinsky ${ }^{4}$ \\ 1. Graduate Program in Genetic Counseling, University of Utah \\ 2. University of Utah Maternal Fetal Diagnostic Center \\ 3. Department of Obstetrics and Gynecology, University of Utah \\ 4. Department of Veterans Affairs
}

Assisted reproductive technology is one of the more regulated medical specialties in the United States; however, there are currently no uniform standards regarding genetic evaluation for oocyte donors. Not only is the genetic evaluation of oocyte donor applicants important for recipients of the gamete, ensuring the highest possibility for reproductive outcome, but also for the donor themselves. Results of these evaluations can have implications for donors, their family members, and their future offspring. To assess genetic screening practices of oocyte donor applicants in the United States an electronic questionnaire was distributed to 202 oocyte donation facilities. The questionnaire inquired about donor family history risk assessment, genetic testing performed on donors, genetic testing consent process, and factors influencing facility policies. Seventy-seven responses were received. Ninety-six percent of facilities assessed donors' family histories and $52 \%$ of these facilities routinely inform donors about the results of these evaluations. Eightyeight percent of facilities reported screening all donors for the same conditions regardless of ethnicity or family history. Sixty-four percent of facilities provide a consultation to donors regarding the genetic screening performed. The majority of facilities report professional organization guidelines are the most influential factor with regards to development of institutional policies regarding genetic screening; however, when reporting what screening is being performed it became clear that most of the facilities are not actually following current guidelines. These data illustrate that there is room for improvement in the implementation of genetic screening practices at oocyte donation facilities. The authors propose development of a consensus guideline with representatives from genetic and reproductive care professionals, which will clarify conflicting guidelines, ensuring high quality reproductive care for all parties involved.

\author{
A Novel Genotype-Phenotype Correlation of a Patient with a 13q13 Deletion: A Case Report \\ M. Nardini' ${ }^{1}$, T. Moss ${ }^{2}$ \\ 1. Cleveland Clinic Foundation \\ 2. Cleveland Clinic
}

We present a seven-year-old female seen at our genetics clinic for the concern of bilateral conductive hearing loss in the context of a past medical history of coarctation of the aorta, bicuspid aortic valve, tethered cord, and short stature. Previous workup at 1 year of age by another genetics clinic for her congenital heart defect and short stature included a normal karyotype and FISH for 22q11.2 deletion syndrome. At age two, she was diagnosed with bilateral conductive hearing loss, which was attributed to abnormally shaped stapes bones. Speech delay was thought to be from the hearing loss, with gross and fine motor development normal. During our genetics evaluation, a chromosomal microarray was ordered. The result was a de novo suspected pathogenic $1.1 \mathrm{Mb}$ deletion in 13q13.3. Included in this deletion were five genes: FAM48A, POSTN, TRPC4, CSNK1A1L, and LINC00547. Previously reported cases of similar deletions consist of slightly different breakpoints $(n=62)$ (Mitter et al. 2011). These similar deletions reported include the RB1 gene, and therefore these patients have all had retinoblastoma, with one exception. Our patient has a novel deletion, which does not include the RB1 gene. Most notably, however, the POSTN gene has been associated with bone formation. We hypothesize that the deletion of POSTN is the cause of our patient's abnormally shaped stapes bones, and consequently her hearing loss. There have been no previous reports of POSTN mutations or deletions causing hearing loss. Our patient's deletion including POSTN and excluding RBI helps define the phenotypic consequences of specific breakpoints among case reports of similar deletions.

\section{Exploring Reasons for Genetic Testing Not Pursued in Patients Referred for Genetic Risk Evaluation}

\author{
J. Osborne ${ }^{1}$, C. Williams ${ }^{2}$, E. Stoffel ${ }^{2}$, J. Everett ${ }^{1}$, V. Raymond ${ }^{2}$ \\ 1. Cancer Genetics Clinic, University of Michigan \\ 2. University of Michigan
}

Introduction: Increasing awareness of the availability of cancer genetic testing and its impact on medical management and cancer screening has impacted referrals to cancer genetics clinics nationwide. However, genetic testing is not indicated for all referred patients, and often reassurance is provided following genetics risk assessment. Understanding genetic risk assessment outcomes is necessary for assessing the appropriate use of genetic testing. Methods: Records for new patients evaluated in the Cancer Genetics Clinic at the University of Michigan between January 1, 2013, and December 31, 2013, were reviewed to determine genetic testing recommendations and outcomes. All patients consented to participation in clinical research. Results: 575 new patients were evaluated and consented during the study period. Genetic testing was not recommended in 210 patients (36.5\%). The most common reasons for not offering testing included: not indicated following genetics risk assessment $(48.6 \%)$ and testing was recommended in an affected family member (28.6\%). Other reasons for not offering genetic testing included: testing was previously completed (14.8 \%) and additional records were requested to complete risk assessment $(8.6 \%)$. Of those patients for whom genetic testing was recommended, the majority completed testing (286/365; 
$78.3 \%)$ with insurance barriers being the most common reason for not completing testing (50/79; 63.3\%). Conclusions: Genetic risk assessment in the setting of formal genetic counseling identifies patients for whom genetic testing may not be appropriate. In this series, more than a quarter of new patients did not have testing due to lack of clinical indication thus providing reassurance or because there was a more informative testing candidate in the family. For those in whom genetic testing is clinically indicated, insurance continues to be a barrier. Formal genetic counseling and risk assessment aids in identifying individuals who would most benefit from clinical cancer susceptibility genetic testing while limiting unnecessary testing.

\section{Confirming Prenatal FISH Results: A Rare Prenatal Diagnosis of Duplication (21) (q22.13q22.2)}

K. Patek ${ }^{1}$, K. Levandoski ${ }^{2}$, L. Briere ${ }^{2}$, L. Ross ${ }^{1}$, K. Kopita ${ }^{1}$, L. Mahon ${ }^{1}$, J. Dayanghirang ${ }^{1}$, J. Jahn ${ }^{1}$, J. Kang ${ }^{1}$, T. Quinlan ${ }^{1}$, A. Vishwakarma ${ }^{1}$, M. El-Naggar' ${ }^{1}$, P. Mowrey ${ }^{1}$, S. Schonberg ${ }^{1}$

1. Quest Diagnostics

2. Beth Israel Deaconess Medical Center

We report a case in which a seemingly straightforward amniocentesis resulted in the identification of a rare cryptic dup(21) (q22.13q22.2). A 30 year old woman, 17 weeks 2 days gestation, elected to have an amniocentesis due to abnormal ultrasound findings of increased nuchal fold and echogenic intracardiac focus. Prior noninvasive prenatal testing (NIPT) was ordered due to increased NT early in the pregnancy; results were normal. Fluorescence in situ hybridization (FISH) and chromosome analysis were ordered on amniotic fluid. FISH aneuploidy screening on direct amniocytes suggested a male fetus with three signals, consistent with trisomy 21 . Apparently paired signals in some interphase cells suggested the possibility of an isochromosome, not noted in the FISH report. Analysis of 20 G-banded metaphase cells showed a male karyotype (46,XY), with two copies of chromosome 21. Due to the discrepant results, FISH for chromosome 21 was repeated on metaphase cells, which yielded significantly enhanced signals from the Down Syndrome Critical Region (DSCR), 21q22.13-q22.2. Subsequent chromosomal microarray results revealed a 3.5 Mb duplication within bands 21q22.13q22.2 extending from nucleotide position [hg19] 38,589,731 to 42,113,758. The duplicated segment is within the assigned DSCR and contains the DYRK1A gene reported to be responsible for much of the neurologic phenotype of Down syndrome. This case demonstrates the necessity of correlating the results of multiple genetic tests to provide a definitive prenatal diagnosis. Additionally, the importance of clear genetic counseling for all testing options and results, with timely communication between clinics and laboratories, is essential for best patient care.

\section{Quality of Care Surrounding Genetic Tests Ordered by Non-Geneticists}

J. Peredo' ${ }^{1}$ I. Lubin², M. Scheuner ${ }^{3}$

1. VA Greater Los Angeles Healthcare System

2. Centers for Disease Control and Prevention

3. VA GLA Healthcare System, D. Geffen School of Medicine UCLA

Objective: We assessed documentation of pre- and post-test medical decision-making for genetic tests (HLA-B27, hemochromatosis, and thrombophilia) ordered by non-geneticists to evaluate the quality of care provided. Methods: We reviewed 265 health records with a genetic test of interest (Apr-Sep 2011). Each record included progress notes 6 months prior to and after test collection. Best practice for pre-test documentation included at least three of the following in a single progress note: indication for testing, test name, family history and informed consent. Best practice for post-test documentation included two of the following in a single progress note: test result, implications to patient and/or family, and management. Regression analyses assessed associations between best practice documentation and patient and provider characteristics, hospital site, and type of test. Results: Mean patient age was 55 years (sd 14.8); $46 \%$ were white, and $90 \%$ were male. Best practice pre- and post-test documentation was found in $21 \%$ and $40 \%$ of records, respectively. Pre-test documentation was more likely given a positive family history (OR 2.16; 95\%CI 1.08-5.03), less likely when $H L A$-B27 (OR 0.09 ; $95 \%$ CI $0.03-0.24$ ) or HFE (OR 0.36 ; 95\% CI 0.14-0.93) tests were ordered compared to thrombophilia testing, and less likely by providers who ordered more tests (OR $0.80 ; 95 \% \mathrm{CI} 0.64-0.99$ ). The only factor influencing post-test documentation was an abnormal test result (OR 4.98; 95\% CI 1.77-14.0). In charts with abnormal results, $23 \%$ (12/52) did not have documentation of results, implications and management. Conclusions: Documentation of medical decision-making by non-geneticists for common genetic tests was low. Pre-test documentation was associated with patient, provider and test characteristics, whereas post-test documentation was only associated with abnormal test results. However, post-test documentation was absent in a large number of charts with abnormal test results. Our findings suggest inefficiencies and potential for poor patient outcomes for commonly ordered genetic tests.

\section{Chromosomal Microarray in Prenatal Diagnosis: The Wayne State University Experience}

\section{K. Rauch ${ }^{1,}$, J. Roberts ${ }^{2}$, J. Abramowicz ${ }^{2}$}

1. Hutzel Hospital

2. Wayne State University Physician Group

Objective: The 2012 National Institute of Child Health and Human Development multicenter trial demonstrated clinical utility of chromosomal microarray analysis (CMA) in the prenatal setting by identifying additional, clinically significant cytogenetic information compared with karyotyping. Our aim was to evaluate the utilization of these services in a high risk ultrasound and genetics clinic over the past several years. Methods: Between 2011 and April 2014, 339 patients met with a board certified genetic counselor and elected invasive prenatal diagnosis (CVS or amniocentesis) for chromosome analysis for various indications (advanced maternal age, abnormal serum screening, ultrasound presence of soft markers or congenital abnormalities, previous pregnancy/child with aneuploidy). Options for chromosome analysis were standard (450 band level, 20 counts) or limited (450 band level, 5 counts) karyotype with whole genome oligo-single nucleotide polymorphism (SNP) CMA (Aglient 180 K GGXChip + SNP). Results: For all 106 invasive procedures in 2011, standard karyotype was performed. In 2012 and 2013, there were 113 and 84 invasive procedures respectively. 
Standard karyotype was performed in 104 (92\%) and limited karyotype with CMA in $9(8 \%)$ in 2012 and 52 (62\%) and 32 (38 \%) respectively in 2013. From January 2014 to April 2014 there were 34 invasive procedures, 15 (44 \%) for which standard karyotype and $19(56 \%)$ for which limited karyotype with CMA was performed.. Conclusion: Based on our experience, the use of CMA in prenatal diagnosis has expanded over the past several years. While the number of invasive procedures decreased from 2011 to 2014, the number of prenatal CMAs increased from zero in 2011 to $56 \%$ of all invasive aneuploidy testing in 2014. Prenatal CMA should be considered because of its ability to provide improved detection of cytogenetic abnormalities beyond conventional karyotyping. However, genetic counseling should be an integral part of offering CMA because of the potential of identifying complex results and clinically uncertain findings.

Family History or Carrier Screening? How Patients are Identified to be Candidates for Preimplantation Genetic Diagnosis for Three Common Conditions: Cystic Fibrosis, Spinal Muscular Atrophy and Fragile X Syndrome

\section{E. Repass ${ }^{1}$, K. Merrion ${ }^{1}$, J. Saucier ${ }^{1}$, D. Clark ${ }^{1}$, M. Maisenbacher ${ }^{1}$, N. Wemmer ${ }^{1}$, M. Westemeyer ${ }^{1}$, B. Pettersen ${ }^{1}$}

\section{Natera, Inc.}

Objective: Determine how patients are identified as candidates for preimplantation genetic diagnosis (PGD) by review of referral indications for three conditions: cystic fibrosis (CF), spinal muscular atrophy (SMA), and fragile X syndrome. Purpose: Given recent recommendations by American College of Medical Genetics and Genomics and American Congress of Obstetricians and Gynecologists, carrier testing for CF, SMA and fragile X are now commonly offered to prenatal and preconception patients with no family history of those disorders. The purpose of this study was to ascertain if more patients are referred for PGD based on family history or carrier screening. Methods: Patient charts were reviewed from PGD cases referred to a single reference lab between June 2010-March 2014. Reasons for referral included family history of the condition, positive results following routine carrier screening, and positive carrier screening due to an infertility work-up. Results: 210 PGD cases referred from 71 in vitro fertilization (IVF) centers were evaluated. Overall, $42 \%$ had a positive family history of the condition, $47 \%$ were identified as carriers on routine carrier screening, $10 \%$ were identified as carriers due to infertility workup, and $1 \%$ were unknown. Referrals for PGD based on routine carrier screening or infertility work-up made up $40 \%$ of cases in 2011, $55 \%$ in 2012, and $68 \%$ in 2013. Referrals for family history made up $58 \%$ of cases in $2011,45 \%$ of cases in 2012 , and $32 \%$ of cases in 2013. Conclusions: Overall, the reasons for referral were evenly split between family history and routine carrier screening. However, a trend towards increased referrals for PGD based on carrier screening was observed over time. In addition, $10 \%$ of patients being picked up by carrier screening through infertility work-up may suggest that carrier screening should become a routine part of any infertility evaluation since these patients may be already pursuing IVF. Because this was a retrospective analysis, there are limitations to this study including potential for skewed data based on the clinics that referred patients to the reference lab each year, sales strategies to increase referrals, and small sample size.

\section{Detection of Uniparental Disomy by SNP Microarray}

\section{B. Rush ${ }^{1}$, E. Williams ${ }^{1}$}

\section{LabCorp}

Addition of single nucleotide polymorphism (SNP) probes to microarrays has allowed detection of copy-neutral changes, such as uniparental disomy (UPD). UPD, the inheritance of both homologues of a chromosome from the same parent, can result in clinically recognizable syndromes in the case of known imprinted genes. Diagnostic testing by methylation or microsatellite based UPD analysis was previously the only testing available for detection of UPD. Long contiguous stretches of homozygosity (LCSH) present in a single chromosome on microarray can be suggestive of UPD. We present data on detection of UPD by SNP microarray. We analyzed 69 cases submitted for SNP microarray. We considered cases for possible UPD based on interstitial LSCH of 20 megabases $(\mathrm{Mb}$ ) or greater and telomeric LCSH of $10 \mathrm{Mb}$ or greater. Based on LCSH identified, including size and location (interstitial vs. telomeric), we identified cases that warranted further study to confirm or exclude UPD. The smallest LCSH in a single chromosome with confirmed UPD was $11.5 \mathrm{Mb}$; the longest LCSH was 247.2 Mb. For cases with excluded UPD, the smallest LCSH was $11 \mathrm{Mb}$; the longest LCSH was $89.9 \mathrm{Mb}$. The rate of confirmed UPD for imprinted chromosomes was 7/22 (31.8\%) and for non-imprinted chromosomes was 15/47 (31.9\%). Careful consideration of the reporting of UPD by microarray should be discussed. Normal arrays without LCSH result with confirmed UPD and arrays with large LCSH result in biparental inheritance. As seen in the trends found in this presentation, UPD reporting criteria may need to be updated. It may need to be based on the percent of chromosome or differ based on size in conjunction with phenotype. As knowledge of UPD is developing, we may find we can improve classification and identification of UPD, testing strategies, and reporting techniques. SNP microarrays are useful for detecting LCSH patterns that may be predictive (but not diagnostic) of UPD. When LCSH detected by SNP microarray suggests UPD, further molecular analysis is necessary for UPD confirmation and determination of parent of origin.

\section{Next-Generation Sequencing Reveals a Novel Duplication in BRCA2}

V. Semenysty ${ }^{1}$, C. Hartshorne ${ }^{2}$, N. Patil ${ }^{2}$, M. Kennemer ${ }^{2}$, M. Rabideau ${ }^{2}$, A. Agbarya' ${ }^{1}$, Y. Kaplan ${ }^{3}$, A. Dvir ${ }^{3}$, K. Jacobs ${ }^{2}$, J. Paul ${ }^{2}$, L. Soussan-Gutman ${ }^{3}$, M. Powers ${ }^{2}$

1. Division of Oncology, Rambam Health Care Campus, Israel

2. InVitae Corp

3. Oncotest-Teva, Teva Pharmaceuticals Industries, PetachTikva

Approximately $50 \%$ of individuals thought to have hereditary breast cancer are found to have a pathogenic variant in either the $B R C A 1$ or $B R C A 2$ genes. Of those with a pathogenic variant, between 5 and $10 \%$ involve large deletion or duplication rearrangements. Traditional analysis for these rare events was performed by multiplex ligation-dependent probe amplification, quantitative PCR or comparative genomic hybridization. We report a case of a 38 year-old Christian Arab woman diagnosed with invasive ductal carcinoma at 30 years of age. She underwent bilateral mastectomy at age 36 years. The 
family history was suggestive of a hereditary cancer syndrome due to early onset breast and ovarian cancer on the maternal side. Next-generation sequencing (NGS) was performed on 29 genes associated with hereditary cancer syndromes. A duplication involving exons 5-11 of BRCA2 was identified using CNVitae, a new software method of detecting copy number variants (CNV) from NGS read count data. To understand the impact of this duplication on the BRCA2 protein sequence we analyzed sequence reads from this region for split-mappings and were able to confirm that this duplication occurred in tandem within the gene. To determine the precise impact on the protein reading-frame, we used PCR amplification on the $5^{\prime}$ breakpoint occurring in exon 11 and Sanger sequence analysis across this junction identified a 13 bp insertion at the breakpoint, followed by sequence correlating to the middle of intron 4 , that then continued into the middle of exon 11 . The duplication is predicted to cause a met to arg change at codon 1594 followed by a frame-shift that ends with a premature truncation at codon 1597 . The truncated protein is expected to result in a loss-of-function; a well-documented mechanism for $B R C A 2$ inherited breast cancer susceptibility. In addition to being a novel duplication, this is the first clinically reported duplication in BRCA2 using these new methods of detecting CNVs.

\title{
Scientific Explanation: When the "False Positive" Noninvasive Prenatal Test Result Is Not False
}

\author{
C. Settler ${ }^{1}$, T. Boomer ${ }^{1}$, N. Teed ${ }^{1}$, J. S. Saldivar ${ }^{1}$, A. Wagner ${ }^{2}$, K. Rauch ${ }^{3}$, E. Lutz ${ }^{4}$, K. Trimmer ${ }^{4}$, N. Dharajiya ${ }^{1}$ \\ 1. Sequenom Laboratories \\ 2. University of Oklahoma Health Sciences Center \\ 3. Wayne State University Physician Group \\ 4. North Texas Perinatal Associates
}

Introduction: The whole genome sequencing approach for noninvasive prenatal testing (NIPT) has the advantage of providing insights into subchromosomal events with high sensitivity and specificity. Discrepant results often have biological explanations, including confined placental or maternal mosaicism, organ transplantation and maternal malignancies - all previously reported. Here we report three cases of subchromosomal findings that explain discordant NIPT results. Methods: Maternal blood samples submitted to Sequenom Laboratories for MaterniT21TM PLUS testing were subjected to DNA extraction, library preparation, and whole genome massively parallel sequencing as previously described. Sequencing data were analyzed using a novel algorithm to detect trisomies and other subchromosomal events. Results: One case reported as trisomy 18 was discrepant with negative FISH testing by amniocentesis. Subsequent karyotyping revealed additional material on $18 \mathrm{p}$, further characterized by microarray as a $12 \mathrm{Mb}$ gain of 18q. Detailed review of the NIPT tracings identified the same duplication. Similarly, a trisomy 13 positive case was reported, where FISH and karyotype results were negative by amniocentesis. Microarray identified a $32 \mathrm{Mb}$ duplication and a $9.5 \mathrm{Mb}$ deletion in 13q consistent with the NIPT result. Again, the detailed tracings mirrored the resolution of microarray. A third case, positive for 45, X also had discrepant FISH results by amniocentesis. Karyotype and microarray revealed a derivative X chromosome resulting in partial monosomy X and partial trisomy 10 . Detailed tracings were consistent with microarray. Conclusion: Clinicians report discordant results when NIPT results are positive but diagnostic testing results are negative. These cases demonstrate that subchromosomal deletions and duplications may explain some discordant results. FISH and karyotyping are often too limited in scope and resolution to be used as a reference for a genome wide assay such as MaterniT21 Plus. Hence, microarray should be considered in resolving such results.

\section{A Case of Hypertrophic Cardiomyopathy: The First with Likely-Significant Alterations in Both TPM1 and TMEM43}

\section{K. Spoonamore ${ }^{1}$, P. Celestino-Soper ${ }^{1}$, T. Lynnes ${ }^{1}$, Z. Wang ${ }^{1}$, H. Lin ${ }^{1}$, Y. Liu ${ }^{1}$, M. Vatta ${ }^{1}$}

\section{Indiana University School of Medicine}

Introduction: Hypertrophic cardiomyopathy $(\mathrm{HCM})$ is primarily a disease of the cardiac sarcomere. The underlying genetic cause can be identified in $50-70 \%$ of affected individuals. When a genetic cause is identified, HCM sequencing panels reveal more than one pathogenic variant in 5-10\% of individuals. Multiple pathogenic variants are usually associated with more severe clinical symptoms and earlier onset. Case Report: We present an adult Caucasian female diagnosed with HCM at age 64 after experiencing shortness of breath, dizziness, sweating, and exertional fatigue. She had asymmetrical septal hypertrophy measuring $2.3 \mathrm{~cm}$ on echocardiogram. Family history included stroke in the mother, sudden death in three maternal relatives, and "heart thickening" and atrial fibrillation in a sibling. Clinical genetic testing using an 18-gene next-generation sequencing (NGS) HCM panel identified a heterozygous c.574 G>A (p.E192K) diseasecausing variant in TPM1. Predictive testing has been pursued in relatives. The patient was enrolled in a research registry and biobank. Research-based NGS testing (targeting coding regions of 246 cardiac-related genes) confirmed the c.574 G>A TPM1 variant and identified an additional heterozygous c.934 C $>$ T (p.R312W) variant in exon 11 of TMEM43, which has been previously reported to co-segregate with arrhythmogenic right ventricular cardiomyopathy (ARVC) and be a founder mutation in Newfoundland (Canada) and the UK. Discussion: Although this patient does not have a clinical ARVC diagnosis, the TMEM43 variant is likely contributing to her cardiomyopathy, establishing what we believe to be the first reported case of digenic HCM involving TMEM43. Relatives with either variant are likely predisposed to cardiomyopathy. Additionally, this case supports the use of larger cardiomyopathy gene panels even with a clear clinical diagnosis of one cardiomyopathy subtype. However, this should be done in the context of careful pre- and post-test counseling, particularly given the number of variants identified and analyzed as part of this larger panel.

\section{Attitudes and Motivations towards Genetic Testing among College-Level Athletics}

A. Squire ${ }^{1}$, K. Valverde $^{1}$

\section{Arcadia University}

In 2010 the National Collegiate Athletic Association (NCAA) implemented sickle cell screening in regulation Division-I health and safety standards. This decision is controversial as sickle cell trait does not typically inhibit athletic performance but rather is important to help prevent possible risks such as collapse and even sudden death. With this NCAA precedent and the rapid growth of personal genomics, genetic testing is quickly becoming an 
integrated piece of sports medicine. The current models of genetic testing are founded in principles of autonomy and informed consent, yet both of these are defined differently in the realm of athletics. The college-level student-athlete is in a unique position; they are not in exclusive control of their own care, and their decisions, as well as subsequent results, could have direct implications for both their current and future careers. The current study surveyed 60 members of the 2014 Temple University Women's Rowing team to determine which factors are most influential in the student-athlete's decision to pursue/consent to genetic testing. Surveys were returned from 45 athletes for a response rate of $75 \%$. Thirty-seven (82.2 \%) reported a preference that testing and possible results be explained in person; thirty-five (77.8\%) reported that they would prefer that the condition being tested for, if positive, would not affect athletic eligibility, and $27 \%$ reported that conditions tested for should have a treatment or cure. Although most of the student-athletes surveyed consider themselves comfortable with genetic testing, the majority also recognizes that they are uninformed on the subject. Student athletes surveyed stated their preferences for in person consultations regarding genetic testing an interesting role for genetic counselors. Continuation of this research would ideally examine a larger and more diverse sample, including males and members of other athletic disciplines.

\title{
From Last to First: Repositioning Genetic Testing in the Diagnostic Process
}

\section{Stanley ${ }^{1}$, H. Hornung ${ }^{1}$, S. Wong ${ }^{1}$, A. Zare ${ }^{1}$, J. Eggington ${ }^{1}$, K. McKernan ${ }^{1}$, R. Boles ${ }^{1}$}

\section{Courtagen Diagnostics}

There has been a longstanding adage in clinical genetics that states, "If a patient has seen as many specialists as they are years or months old, then it is time to refer them to a genetics clinic." The traditional genetics clinic allowed for a genetic diagnosis by skilled geneticists and genetic counselors in determining whether the patient meets certain diagnostic criteria and provided justification for ordering genetic testing. One problem with this process is the ascertainment bias involved in the development of diagnostic criteria, which effectively removes patients with atypical presentations from diagnosis and restricted the phenotypic spectrum of disease. In addition, single gene sequencing had the effect of extending the time and cost to reach a genetic diagnosis. This approach was dictated in part by the cost associated with Sanger sequencing, where significant clinical justification was required to add a new gene into a panel. Massive parallel sequencing brought both a radical drop in cost-per-gene and the advent of large comprehensive gene panels, allowing individuals who do not classically fit diagnostic criteria to be evaluated for genes with or without high clinical utility. Suddenly, primary care physicians are ordering genetic tests on patients who only meet a subset of diagnostic criteria, which has moved genetics to the beginning of the testing process, effectively turning the traditional process on its heels and providing a shortcut to what has been often a long maze of referrals. This provides both challenges and opportunities to both physicians and genetic counselors; empowering families with genetic information at a time when potential treatments can be effective, specific agents can be avoided, and awareness brought to potential underlying medical issues. To demonstrate the clinical utility in performing large panel genetic testing, cases will be presented, including cerebrotendinous xanthomatosis, Cardio-Facio-Cutaneous syndrome and Angelman syndrome, with focus on the benefits of early diagnosis.

Rethinking the Family Cancer History Questionnaire in the Era of Next Generation Sequencing Panels: Are We Asking the Right Questions? K. Vikstrom ${ }^{1}$, M. Blundell ${ }^{2}$, B. Vikstrom ${ }^{3}$, S. Topper ${ }^{4}$, T. Ekstein ${ }^{4}$, M. Powers ${ }^{1}$

1. Invitae Corporation

2. Sutter Cancer Center

3. Northbay Cancer Center

In medical and surgical oncology settings, the family history questionnaire is often used as a screening tool in identifying patients who may benefit from a genetics evaluation. Studies have shown, however, that inherited cancer syndromes remain under-diagnosed, largely because clinicians often limit their investigation to immediate family members and do not document cancers in extended relatives. Recent studies have also demonstrated that oncologists lack confidence in their ability to interpret risk from family history, particularly in cases where the patient presents with a rare cancer or has an unusual pattern of cancer in his or her family. Moreover, many providers focus on collecting family history data specifically relating to cancers associated with the more common conditions, such as Hereditary Breast and Ovarian Cancer syndrome, and may not recognize overlapping features of other syndromes. The authors propose that a family history questionnaire designed to cast a wider net and be inclusive of a larger number of inherited cancer syndromes is useful, now that panels are available and next generation sequencing allows for the investigation of many genes at once. Specifically, we propose a twostep questionnaire (a simple form for patients and a follow-up form for the clinician) that is designed to guide the patient and clinician to investigate family history by systematically reviewing major organ systems/tumor sites of affected relatives. This "check-list" format leads to a preliminary differential diagnosis and an associated condition/gene list, allowing for consideration of multiple conditions at once, and may be a time-saving way to collect more detailed family history data.

\section{Evaluation of Laboratory Perspectives on Multiplex Genetic Testing for Hereditary Cancer Susceptibility by Next Generation Sequencing}

\author{
J. Stoll ${ }^{1}$, S. Weissman ${ }^{2}$, N. Hook ${ }^{3}$, A. Knight Johnson ${ }^{1}$, A. Newlin ${ }^{3}$, C. Selkirk ${ }^{3}$, K. J. Vogel Postula ${ }^{2}$ \\ 1. University of Chicago \\ 2. GeneDx, Inc. \\ 3. NorthShore University Health systems
}

Genetic counseling for hereditary cancer susceptibility is an evolving field, continually adjusting to advancements in genetic testing technology. New next-generation sequencing technologies are rapidly changing the way hereditary cancer genes are being assessed by allowing simultaneous analysis of numerous cancer susceptibility genes. This study explores the laboratory perspective on multiplex panels for cancer susceptibility genes. Semi-structured interviews were conducted with representatives from five clinical laboratories offering hereditary cancer gene panels. Interviews were analyzed using an inductive analysis approach, and themes were established to describe the laboratories' perspectives on multiplex panels. Several themes were identified, 
including similarities and differences between laboratories pertaining to development of cancer gene panels, communication of panel properties and follow-up with clinicians and patients, variant policies, and the future of hereditary cancer gene testing. All laboratories discussed clinical utility as a main contributor to the decision processes behind panel development. Additionally, while many participants indicated that gene and syndrome overlap prompted panel development, laboratories differed in their opinion of whether or not phenotypes of these syndromes overlap enough to justify offering a pan-cancer panel exclusively. Participants also stressed the importance of patient understanding of panel testing, including what they are being tested for and the limitations of testing. While all laboratories discussed the limitations of a variant of uncertain significance result, they differed significantly in how they report variants of uncertain significance results. Overall, this study serves to provide healthcare providers with information on how laboratories approach panels, highlighting universal commonalities as well as fundamental differences. This may allow ordering providers to think more critically about these tests, thus improving their ability to make informed decisions when ordering cancer gene panels.

\section{International Data Sharing Efforts: Lessons from Canadian Open Genetics Repository}

S. White ${ }^{1}$, S. Baxter ${ }^{1}$, M. Oates ${ }^{1}$, C. Graham ${ }^{1}$, J. Lerner-Ellis ${ }^{2,3}$, M. Lebo ${ }^{1,4}$,

1. Partners Personalized Medicine

2. Mount Sinai Hospital

3. Ontario Institute for Cancer Research, University of Toronto

4. Bringham and Women's Hospital

Individual laboratory knowledge and siloed data sets impede our knowledge of variants and prevents clinicians from receiving the most accurate interpretations for variants found in their patients. This in turn may prevent patients from receiving the most appropriate care. Furthermore, due to the increasing number and breadth of genetic tests there are a surge of variants of uncertain significance being returned. The Canadian Open Genetics Repository (COGR) is a collaborative effort for the collection, storage, sharing and robust analysis of variants reported by medical diagnostics laboratories across Canada. As clinical laboratories adopt modern genomics technologies, the need for this type of collaborative framework is increasingly important. The aim of this initiative is three-fold. First, design a freely available, robust and consistent variant assessment tool. Second, transfer individual labs' variant, gene and disease knowledge to a common application, GeneInsight, so labs can curate and share data via a unified platform. Lastly, enable real-time data sharing between clinical labs to enhance the exchange of information about novel variants within the expert community. This allows for aggregation of variant knowledge across sites so patient advocacy groups and the public can access expert curated consensus agreements on variant interpretations. We present here the experience, difficulties and lessons learned initiating this collaborative effort. The initial COGR network currently contains over 3,000 variants across 52 genes which are associated with 6 diseases including hereditary breast and ovarian cancer and hypertrophic cardiomyopathy. In total, 391 variants have been identified in more than one lab including 50 that have been identified by three labs. As an ongoing endeavor, COGR serves as focal point for the collaboration of Canadian laboratories with other international efforts in the development of tools and methods to take full advantage of clinical laboratory data.

\section{Multi-Gene Testing for Paragangliomas and Pheochromocytomas: Diagnostic Yield and Phenotypic Spectrum}

S. Witherington ${ }^{1}$, C. Horton ${ }^{1}$, J. Dolinsky ${ }^{1}$

\section{Ambry Genetics}

Paragangliomas (PGL) and pheochromocytomas (PCC) have recently proven to exhibit genetically heterogeneous etiologies, leading to the proposal of several algorithms for sequential or concurrent testing. We sought to determine the phenotypic spectrum of mutation carriers and diagnostic yield of a multi-gene test encompassing PGL/PCC genes including genes traditionally correlated with syndromic diseases. DNA from 91 individuals with a personal or family history of PCC, PGL, or related cancers was analyzed with a multi-gene test including next generation sequencing and deletion/ duplication analysis of MAX, NF1, RET, SDHA, SDHAF2, SDHB, SDHC, SDHD, TMEM127, and VHL. All clinical information was obtained from test requisitions. $95.6 \%(n=87)$ of probands tested were evaluated by genetics professionals; $87.9 \%(n=80)$ had a personal history of either PCC or PGL with an average age of onset of 39 years of age. Pathogenic mutations were identified in $22(24.18 \%)$ individuals and variants of uncertain significance (VUS) were identified in $10(10.99 \%)$ individuals. The highest number of mutations were identified in $S D H B(n=11)$ followed by $S D H A(n=3)$, accounting for $63.6 \%$ of total mutations identified. Two probands had a mutation in VHL and one proband had a mutation in RET, none of whom exhibited syndromic features. No mutations were identified in patients diagnosed between age 30-39 or those diagnosed over the age of 60 . Two of three probands with gastrointestinal stromal tumors (GIST) had an SDHx mutation. These results lend support for the inclusion of genes traditionally considered in individuals with syndromic presentations $(V H L, R E T)$ in a multi-gene test for PGL/PCC. Observation of $S D H x$ mutations in probands with GIST emphasizes the phenotypic and allelic overlap between hereditary PGL-PCC syndrome and Carney-Stratakis dyad. Overall, PGLNext has the highest diagnostic yield of all the large multi-gene tests available at Ambry Genetics, suggesting a role for multi-gene testing in clinical management of all individuals with PGL/PCC.

\section{Utilization of Next Generation Panel Genetic Testing Diagnoses a Patient with Atypical Colon Polyp Presentation}

\section{Williams ${ }^{1}$, V. Raymond ${ }^{1}$, E. Stoffel ${ }^{1}$, J. Carethers ${ }^{1}$, T. Else $^{1}$, J. Everett ${ }^{2}$}

1. University of Michigan

2. Cancer Genetics Clinic, University of Michigan

Introduction: The implementation of next-generation sequencing (NGS) panel testing allows simultaneous investigation of multiple cancer syndromes with overlapping clinical features. This case demonstrates the use of NGS panel testing in a patient with an atypical clinical presentation, providing a genetic diagnosis which may have been missed using single gene testing methods. Case Report: A 31 year old male presented to the emergency 
department with dizziness. Initial workup identified anemia and positive stool guaiac. Colonoscopy identified a $4 \mathrm{~cm}$ ( $\mathrm{cm}$ ) ascending colon lesion, with biopsy noting adenomatous tissue. Post-colonoscopy course was complicated by agitation and confusion. Head CT revealed a right major cerebellar stroke. Follow-up colonoscopy re-identified the $4 \mathrm{~cm}$ lesion as well as $1.5 \mathrm{~cm}$ and $0.5 \mathrm{~cm}$ ascending colon polyps. All three lesions were resected and histology showed adenomas. Patient subsequently presented for clinical genetics evaluation, and reported a sister with colon polyps and anemia. The differential diagnosis included Lynch syndrome and adenomatous polyposis conditions. Given clinical overlap, he was offered an NGS panel for colon cancer and polyp susceptibility genes which identified a SMAD4 deletion (exons 1-9), consistent with a diagnosis of Juvenile Polyposis syndrome (JPS) and raising concern for the overlap syndrome with Hereditary Hemorrhagic Telangiectasia. Brain MRI showed no evidence of arteriovenous malformation (AVM). Contrast echocardiogram showed a delayed right-left shunt suggestive of possible pulmonary AVM. Discussion: Diagnostic criteria for hamartomatous polyposis syndromes, including JPS, require presence of at least one hamartoma. This patient's polyps were histologically adenomas, with one small hamartoma appreciated on retrospective review after JPS diagnosis. Panel testing enables genetic diagnosis of patients with atypical polyp histories, decreasing reliance on prospective polyp count and histology. Panel testing also expands the phenotypic spectrum of polyposis syndromes.

\title{
Pediatrics
}

\section{Biochemical and Clinical Findings of Patients with the Common Variant (C.625g >A) in ACADS Identified through Minnesota Newborn Screening}

\author{
M. Shearouse ${ }^{1}$, C. Alexander ${ }^{2}$, B. LeRoy ${ }^{3}$, P. McCarthy-Veach ${ }^{1}$, K. Bentler ${ }^{4}$, S. Elsbecker ${ }^{4}$, S. Berry ${ }^{4}$ \\ 1. University of Minnesota \\ 2. University of Minnesota Medical Center, Fairview \\ 3. Department of Genetics, Cell Biology and Development, University of Minnesota \\ 4. Department of Pediatrics, Genetics and Metabolism, University of Minnesota
}

The clinical significance of short-chain acyl-coenzyme A dehydogenase deficency (SCADD) has been suspect since the expansion of newborn screening programs and subsequent identification of asymptomatic individuals. It is thought that the common $A C A D S$ variant c.625G $>$ A contributes to the biochemical features of SCADD, but the variant alone is not sufficient to cause symptoms. The variant allele frequency is $22 \%$ in the U.S. population. SCADD is a relatively rare condition, with an estimated prevalence of 1 in 25,000 in Minnesota. Consequently, there is a paucity of published information about the characteristics of SCADD patients identified by newborn screening and the contribution of ACADS genotype. The purpose of the present study is to describe the biochemical findings and clinical phenotypes of patients who were ascertained by Minnesota Newborn Screening and have the c. $625 \mathrm{G}>\mathrm{A}$ variant in $A C A D S$ with or without other sequence alterations in the same gene. Chart review was conducted of patients who visited the University of Minnesota for elevated C4 identified by Minnesota Newborn Screening from 2001 through 2013. An investigator-developed rubric was used to extract data from the charts. Results indicated 31 patients whose $A C A D S$ sequencing revealed the c. $625 \mathrm{G}>\mathrm{A}$ variant in addition to another sequence alteration in the same gene: eight were homozygous for the variant alone; eight were homozygous for the variant and had an additional $A C A D S$ sequence alteration; and 15 had one copy of the variant with another sequence alteration in $A C A D S$. Three of 31 patients studied had symptoms that have been associated with SCADD. There is no apparent association between genotype and clinical phenotype. The present study reports the largest group of patients $(n=31)$ ascertained by newborn screening who had molecular analysis of ACADS in their work up for SCADD. The results support prior research findings that few patients with SCADD identified by newborn screening manifest symptoms and $A C A D S$ genotype is not associated with the occurrence of symptoms. Additional findings, practice implications, and research recommendations are presented.

Hemophilia B Acquired from Liver Transplantation: A Case Report and Literature Review

\author{
K. Bergstrom ${ }^{1}$, A. Stevens ${ }^{2}$, L. Srivaths ${ }^{3}$, D. Yee $^{3}$ \\ 1. Department of Pediatrics, Baylor College of Medicine \\ 2. Department of Pediatrics, Hematology-Oncology Section, Baylor College of Medicine \\ 3. Baylor College of Medicine
}

Hemophilia B, an inherited bleeding disorder, occurs in about one in 25,000 male births. Hemophilia B is caused by a mutation in the F9 gene, which results in a deficiency of coagulation factor IX. This critical coagulation factor is synthesized by hepatocytes in the liver. Individuals with hemophilia B who develop severe liver disease from infectious causes are cured of both the liver disease and hemophilia through orthotopic liver transplantation (OLT). In contrast, we report a case of hemophilia B acquired from OLT, confirmed by genetic testing. A 28-month old Caucasian male was referred to the hematology service for consultation due to a history of easy bruising and persistently prolonged activated partial thromboplastin time (aPTT). Factor IX levels were measured at $8 \%$. The past medical history was significant for a cadaveric OLT due to biliary atresia. The patient had normal aPTT values and no bleeding or bruising problems (including a circumcision without bleeding) prior to OLT. F9 gene sequencing on the patient's leukocytes was normal. F9 gene sequencing performed on a sample of DNA isolated from the transplanted liver tissue identified a mutation not found in the patient's blood sample. This mutation has been identified in other patients with mild hemophilia B, and appears to be more common in the African American population. Interestingly, the liver donor was a 14 year old African American male who died of head trauma. This is the first reported case of hemophilia B acquired via OLT. In our patient's case, mild hemophilia B was not previously reported in the donor and only became recognized as an acquired disorder in the liver transplant recipient after he developed easy bruising and was found to have prolonged PTT and low factor IX level. This case illustrates that a bleeding disorder can be inadvertently transmitted via OLT. The possibility of this transplant related complication deserves consideration and inclusion in the risk-benefit analysis of liver transplantation and highlights the importance of careful organ donor screening practices. 
Development of Newborn Screening Connect: A Self-Reported Patient Registry and Its Role in Improvement of Care for Inherited Metabolic Disorders

\author{
A. Devarajan ${ }^{1}$, Y. Osara ${ }^{1}$, K. Coakley ${ }^{1}$, R. Singh ${ }^{1}$
}

\title{
1. Emory University
}

Newborn Screening Connect (NBS Connect) is a web-based self-reported patient registry designed as a resource for individuals and families affected by inherited metabolic disorders included in the newborn screening panel. NBS Connect was launched in 2012 by Emory University after years of planning and grassroots work by professionals, consumers, and industry. Individuals with phenylketonuria (PKU) or maple syrup urine disease (MSUD) were recruited through distribution of outreach materials to metabolic clinics, presentations at parent organization meetings, newsletters and direct recruitment at clinic appointments. Participants completed their profiles to generate data on diagnosis, treatment, symptoms, outcomes, barriers to care, and quality of life. Furthermore, to examine the ability of NBS Connect to generate data to guide hypothesis-driven research, data pertaining to age at diagnosis, bone and skin health in individuals with PKU were assessed. As of March 2014, NBS Connect had 179 registered participants: 128 (72\%) individuals with PKU, 19 (11\%) individuals with MSUD, and 32 (18\%) individuals with other NBS disorders. For all registry participants, age at diagnosis was less than 4 weeks in $81(83 \%)$ individuals and between 1 month and 14 years in 15 (16\%) individuals. Among 102 respondents with PKU, 10 (10\%) individuals had a bone density scan in the past year and 92 individuals did not. Of 100 respondents, $40(40 \%)$ individuals had at least one skin disorder indicating a higher prevalence compared to the normal population (12.4\%). Based on data from NBS Connect, future studies can be initiated to test hypotheses such as the relationship between PKU and skin disorders. Patient registries like NBS Connect can inform hypothesis-driven research, contributing to knowledge generation. This strategy keeps in pace with the current trend in moving from traditional medicine towards evidence-based practice. Participation and contribution in such registries helps in understanding long-term outcomes of rare disorders, contributing to better patient care and quality of life.

\section{Pediatric Neurometabolic Disorders: Medical Needs, Service Use and Disease Impact on the Family}

\author{
J. Diaz ${ }^{1,2}$, C. Spaeth ${ }^{2}$, B. Hallinan², V. Pilipenko², T. A. Burrow ${ }^{2}$
}

\section{University of Cincinnati}

2. Cincinnati Children's Hospital Medical Center

Background: Inborn errors of metabolism include many rare genetic disorders with significant morbidity and mortality. Neurometabolic diseases, a subtype of these disorders, have a variable presentation with both neurologic and non-neurologic symptoms. Presently, few evidence-based guidelines exist to standardize patient care across centers and providers. Due to the absence of such recommendations, the purpose of this study was to characterize the medical needs, service use, and family impact of pediatric neurometabolic disease. Methods: The study population was comprised of primary caregivers of children (ages 0-17) managed in a neurometabolic clinic at Cincinnati Children's Hospital. Study invitations were sent to 132 eligible caregivers to participate in the online survey. Results: In total, 25 surveys were included in analysis resulting in a participation rate of $19 \%$. Known diagnoses were reported for $60 \%$ of children with the most common being mitochondrial disorders, metachromatic leukodystrophy, and Batten disease. The most frequent medical problems were due to neurological (88\%) and musculoskeletal symptoms $(80 \%)$. Health concerns and functional limitations resulted in increased needs for services such as therapies (100\%), educational supports $(85.7 \%)$, and specialty medical care (75\%). Financial stress (67\%) was the most commonly reported factor impacting caregiver stress level. Many support services were desired by the cohort with $60 \%$ indicating they would like to be involved in support groups. Conclusions: This study provides evidence to better inform the care and genetic counseling needs of children with neurometabolic disorders and their families. The data delineates the medical needs and service use of this population, supporting the idea that multidisciplinary clinics and collaborative care may help to comprehensively meet their complex needs. Caregivers of children with neurometabolic disorders face a significant burden of care and could benefit from increased availability of support services.

\section{If the Microarray Diagnosis Doesn't Quite Fit, You Must Not Quit: A Case of 16p11.2 Deletion}

\section{H. Dubbs ${ }^{1}$, F. Reynosis ${ }^{1}$, E. Zackai ${ }^{1}$}

\section{Children's Hospital of Philadelphia}

Chromosome microarray (CMA) has replaced karyotype as a first tier test in individuals with autism or intellectual disability (ID). The growing use of this technology in these populations has led to the identification of several recurrent microdeletion syndromes associated with ID that lack clinically recognizable phenotypes, such as deletions of $16 \mathrm{p} 11.2,1 \mathrm{q} 21.1$ and $15 \mathrm{q} 13.3$. These deletions are found at high frequency within these populations, demonstrate a range of phenotypic variability with minimal physical manifestations and show incomplete penetrance, making it difficult to know whether a rare presentation represents a novel feature of the disorder, an atypically severe case or is due to a separate etiology. However, making an accurate genetic diagnosis has significant implications for counseling and management. We report a 15 year old male who had CMA testing due to microcephaly, ID and tremor. He was nonverbal and needed assistance with activities of daily living. CMA identified a recurrent $551 \mathrm{~kb}$ deletion of 16p11.2, associated with DD, ID and/or autism spectrum disorder; reported IQs range from 40 to normal, and language deficits are common. These features do overlap with those in the patient, but his tremor, microcephaly, lack of speech and degree of impairment were unusual. This prompted further workup, and UBE3A analysis for Angelman syndrome (AS) identified a pathogenic mutation, p.Tyr471X. Confirming a dual diagnosis of $16 \mathrm{p} 11.2$ deletion and AS allowed us to more accurately counsel the family about prognosis and management. There are some medications that can have negative effects in AS, which could impact pharmacologic treatment. Also, both $16 \mathrm{p} 11.2$ deletions and UBE3A mutations can be de novo or inherited from a parent with a $50 \%$ recurrence risk, influencing reproductive decision making. This case highlights the need to fully investigate atypical/novel features of rare disorders, particularly in those where the phenotypic spectrum is not well defined. 
Perceptions of Severity of Children's Bleeding Disorders: Impact on Parental Quality of Life and Reproductive Decisions

\title{
E. Holt ${ }^{1}$, E. Crocker Lisi ${ }^{2}$, L. Baumanis ${ }^{3}$, S. DeBrosse ${ }^{3}$, A. Matthews ${ }^{4}$
}

1. Cancer Treatment Centers of America

2. Department of Human Genetics, Emory University

3. University Hospitals Case Medical Center

4. Case Western Reserve University

Research results have been mixed regarding the impact of clinically-defined severity on the quality of life and reproductive decisions for parents of children with bleeding disorders. One hypothesis for this discrepancy is that parents may not view the severity of their child's condition similarly to the clinical definition of severity (\% factor level). The purpose of this study was to investigate the influence of parental perception of severity on parents' quality of life and reproductive decisions. Parents with a child who received care at one of three Hemophilia Treatment Centers completed a survey regarding perceived severity, demographics, reproductive decisions, and caregiver quality-of-life (CQOL). Results showed that the majority of participants $(n=126)$ believed their child's bleeding disorder was mildly severe ( $69.0 \%$ ), and rated their child's condition milder than the clinical definition of severity for hemophilia ( $63 \%$ perceived mild vs. $18 \%$ mild by clinical definition). On $t$-test analysis, mean scores for overall CQOL were poorest for participants who perceived their child as severely affected and best for the perceived mild group $(p=<0.001)$; however, perceived severity was not a significant predictor of quality of life. Similar findings were noted for clinically-defined severity. Of the CQOL subscales, only parental burden was impacted by perceived severity, (those who perceived their child's bleeding disorder as more severe, experienced more burden due to the disorder). Perceived severity had little impact on reproductive decisions as $71.4 \%$ stated that their child's bleeding disorder did not influence reproductive plans. Clinically-defined severity was also found not to be a significant predictor of reproductive decisions. Overall, study results suggest that perceived severity may be important for genetic counselors to consider when assessing the well-being of parents of children with bleeding disorders.

\section{Reference Growth Curves for Children with Classic Galactosemia}

\author{
J. Howell ${ }^{1}$, M. Anderson ${ }^{1}$, K. Lai ${ }^{2}$, J. L. Chaloner ${ }^{3}$, K. Wierenga ${ }^{1}$ \\ 1. University of Oklahoma Health Sciences Center \\ 2. University of Utah \\ 3. Perinatal Center of Oklahoma
}

Classic galactosemia (CG) is an inborn error of galactose metabolism that can result in life threatening conditions if left untreated. Despite early diagnosis and compliance with a galactose-restricted diet, long-term complications are frequently seen in patients with CG including speech dyspraxia, ataxia, and primary ovarian insufficiency. Decreased height and weight in children with CG have been reported in few studies, but only one focused on growth velocities. This study aims to determine if there is a significant difference between the growth of children with CG and the Centers for Disease Control (CDC) clinical growth curves. Growth data was collected including height/length, weight, and head circumference for 28 individuals with CG (13 males, 15 females). Ages ranged from birth to 21 years; however, the majority of growth data collected were concentrated from birth to 2 years. Mean length/height, weight, and head circumference were plotted with $95 \%$ confidence intervals. The 50th centiles of CDC clinical growth curves were plotted on the same graph for comparison. Female height at 1,3 , 64, 120 months and male height at 1, 2 and 15 months is significantly lower than the CDC 50th centiles. Similarly, male weight at 1, 2, and 15 months is significantly lower and from 4 to 14 months there is no significant difference. For females, mean weights at months 1-6, 19, 22, 32-35, 43-44, 50, 53, and 72 are significantly lower than the CDC curves and for all other months there is no significant difference. The data show significant differences between the mean heights and weights of CG patients compared to the CDC growth curves. This data indicates that both males and females with CG have growth deficiencies in the first 2 months of life and may also experience growth deficiencies into childhood. These data raise the need for larger growth studies in children with CG, and, if confirmed, studies regarding etiologies and towards improved management.

Discontinuing Enzyme Replacement Therapy in Patients with Lysosomal Storage Diseases Due to Significant Clinical Decline

\author{
A. Kim ${ }^{1,2}$, J. Lynch ${ }^{1}$, M. Walker ${ }^{2}$, L. Berry ${ }^{3}$ \\ 1. University of Cincinnati \\ 2. Cincinnati Children's Hospital Medical Center \\ 3. Cincinnati Children's Hospital
}

Enzyme replacement therapy (ERT) is considered an effective treatment for patients with lysosomal storage diseases (LSDs). Scientific focus has been on the efficacy of ERT; however, as the use of ERT has become more widespread, the issues of when and for whom to consider discontinuation of ERT have arisen and are in need of consideration. The purpose of this study was to describe the experiences, thoughts, attitudes, and behaviors of healthcare providers related to discontinuing ERT in patients with severe forms of LSDs who experience significant clinical decline. Providers' definitions of significant clinical decline in this patient population were also sought. A questionnaire was sent to healthcare professionals through the Metab-L and the NSGC listservs. A total of 81 responses were analyzed. The most commonly selected feature describing significant clinical decline was persistent vegetative state $(58 / 74 ; 78 \%)$. Cognitive decline was a factor as well, when participants responded to two hypothetical patient scenarios. Almost $25 \%$ of participants had previously recommended discontinuing ERT due to significant clinical decline with nearly $90 \%$ of patients opting to discontinue. Recommending discontinuation of ERT showed positive correlation with healthcare providers' number of years of experience. Genetic counselors were less likely to discuss the option with the patient/family compared to the physicians and one of the reasons given was that recommending discontinuation of ERT was outside their scope of practice. This study indicates that healthcare providers are discussing the option of discontinuing ERT for patients with significant clinical decline. Persistent vegetative state and cognitive decline are significant factors under consideration with discontinuing ERT, although the decision should come with full involvement of the patient, family, and healthcare team. Discussion of genetic counselors' roles and scope of practice at the clinic and professional organization levels could enhance engagement with patients and ultimately improve patient care. 


\title{
Low Adiposity of Cystic Fibrosis Mice Due to Small Adipocyte Size
}

\author{
J. Klavanian', R. Darrah', I. Bederman², M. Drumm ${ }^{1}$, A. Matthews ${ }^{1}$ \\ 1. Department of Genetics and Genome Sciences, Case Western Reserve University \\ 2. Department of Pediatrics, Case Western Reserve University
}

Low adipose stores have been previously demonstrated in cystic fibrosis (CF) patients and mice. It is not yet known whether this adipose tissue deficit is due to fewer adipocytes present in CF mice or due to a morphological adipocyte problem inhibiting fat from properly entering and/or staying in the cell. The purpose of this study was to physiologically characterize the nature of the adipose tissue defect present in mice with CF. Methods: Studies were performed on F508del (Cftrtm1kth) mice and littermates homozygous for the wild-type (WT) CFTR allele. Gonadal fat pads were removed and weighed from CF and age matched WT mice. Sections of CF and WT adipose tissue were viewed histologically to observe adipocyte size. DNA and triglycerides (TG) were extracted from gonadal fat pads and quantitated. Results: CF mouse adipocytes were shown to be smaller, based on their observed and quantitated differences viewed histologically. CF mice had significantly more adipose cells per frame vs. WT mice; $(p=0.0002)$ demonstrating a 2 -fold increase; DNA concentration was also increased (a 2-fold higher concentration of DNA per gram of adipose tissue than WT mice, $p=0.0023$ ); comparable amounts of total adipocytes were present in CF gonadal fat pads compared to WT mice, and there was less TG content per adipocyte (CF mice had 2.5-fold less TG content per fat pad, $p=0.009$ ). Since CF and WT mouse fat pads have similar numbers of cells, these data demonstrate that the deficit in fat is from smaller adipocytes. Conclusions This study provides novel evidence that low adiposity in CF mice is caused by small adipocyte size. It is important for genetic counselors to better understand fat metabolism in CF patients in order to provide appropriate management recommendations and anticipatory guidance.

\section{Carrying Cri-Du-Chat: A Case Report of 46, XX, Del (5) (p15.32)Mat \\ K. Lammers ${ }^{1}$, H. Meng ${ }^{1}$, M. Miller ${ }^{1}$, J. Wiley ${ }^{2}$}

\section{Dayton Children's Hospital}

2. East Carolina University

Introduction: The 5p- microdeletion syndrome, better known as cri-du-chat, is characterized by intellectual disability, dysmporphic facies, speech delay, and a cat like cry. It is believed that around $85 \%$ of cri-du-chat cases are de novo while the remaining cases develop secondary to unequal segregation of parental translocation or, less commonly, inversion. Cases of inherited 5p- syndrome are rare in the literature. Here we report a maternally inherited case of cri-du-chat. Case Description: A 9 year old African American female presented at clinic to establish care based on her previous diagnosis of Cri-duchat. Chromosome analysis was completed when she was 4 years old after a dentist noted abnormalities and microcephaly. The child has delayed developmental milestones, currently has between 50 and 100 words, and has a head circumference 2 standard deviations below the mean. After the appointment we obtained the cytogenetic report of the child which showed; 46, XX, del (5) (p15.32)mat. Both the mother and daughter have had comparative genomic hybridization microarrays to confirm the $5.4 \mathrm{Mb}$ terminal deletion. Mom was unaware she carries this deletion, and we met to answer her questions. It was reported that mom attained her motor and verbal milestones on time but had a high pitched cry. Mom feels her high pitched voice persists. While schooling was difficult for mom she was able to graduate high school, some college, and spent time working as a substitute teacher. Discussion: This case demonstrates the phenotypic variability of $5 \mathrm{p}$ - syndrome between family members with identical deletions. Noteworthy, the child has significant speech delay while the mother is able to clearly and easily express herself verbally. Screening of parents of children with $5 \mathrm{p}$ - may be warranted, even in the case of a normal parental phenotype, to assist with accurate recurrence risk assessment.

\section{Case Series: Eliminating Barriers to Genetic Testing in the Duchenne/Becker Muscular Dystrophy Community}

\author{
A. Martin ${ }^{1}$, A. Lucas ${ }^{2}$, M. Hegde ${ }^{3}$, H. Peay ${ }^{1}$ \\ 1. Parent Project Muscular Dystrophy \\ 2. DuchenneConnect Registry, Parent Project MD \\ 3. Emory Genetics Laboratory
}

Objective: Decode Duchenne is a program of DuchenneConnect that provides genetic testing to U.S. Duchenne/Becker muscular dystrophy (DBMD) patients unable to access testing. DuchenneConnect is a self-report registry for DBMD patients. With mutation-specific therapeutics in trial, genetic testing is increasingly important, but financial limitations can be a barrier. Decode Duchenne is funded by Sarepta Therapeutics. Methods: Patients with no genetic testing or inconclusive testing were recruited. Emory Genetics Laboratory performed Duchenne Muscular Dystrophy (DMD) CGH array analysis to identify deletions/duplications. If negative, DMD sequencing was performed. If sequencing was negative, the Neuromuscular Gene Panel was ordered, which screens for mutations in 46 genes associated with neuromuscular disorders. Results: To date, we received applications from 30 patients and results on 18. Ten previously had inconclusive testing while 20 had no previous genetic testing. Most providers reported testing was denied by Medicaid or private insurance. Nine patients were found to have a deletion and 1 had a duplication. Sequencing was obtained on 8 patients and completed in 6. Sequencing identified a nonsense mutation in 2 patients and a variant of unknown significance in 1 patient. Sequencing was negative on three patients with results from the Neuromuscular Gene Panel currently pending. Conclusion: Mutation-specific therapy trials require genetic confirmation for participation. Obtaining testing has been difficult for some patients. While genetic testing is standard of care, some insurance providers do not cover testing. Genetic professionals must continue advocating for patients and educating the insurance community on the necessity of genetic testing. Our results also highlight the utility of genetic testing to verify a diagnosis. Three out of 18 cases have no mutation identified by sequencing, which is unexpected. This is important for potential therapeutic interventions and reproductive risk counseling. The Decode Duchenne program provides access to genetic testing and highlights areas for future study. 
When Café Au Lait Spots Do Not Equal NF1: Constitutional Mismatch Repair Deficiency as an Important Differential Diagnosis for NF1 Stigmata and Pediatric Cancer

\author{
R. McGee ${ }^{1}$, M. Walsh ${ }^{1}$, C. Odom ${ }^{1}$, S. Jeha ${ }^{1}$, A. Pappo ${ }^{1}$ \\ 1. St. Jude Children's Research Hospital
}

Constitutional mismatch repair deficiency (CMMRD) syndrome is a newly described condition involving bi-allelic mismatch repair (MMR) gene mutations in MLH1, MSH2, PMS2 or MSH6. This syndrome is characterized by pediatric-onset of hematological, brain, Lynch syndrome-associated and other cancers. A 12-year-old girl was referred to our genetics clinic for evaluation of café au lait spots (CALs) and a history of acute lymphoblastic leukemia (ALL) diagnosed at age 10 years. Evaluations revealed $>6$ CALs $>5 \mathrm{~mm}$ in size on the patient's neck, back and trunk; small subcutaneous nodules on left thigh and behind right ear; and one iris nevus in the left eye with no Lisch nodules. Family history was significant for a paternal first cousin who died of a brain tumor at age 27 and maternal history of colon polyps. Consanguinity was denied. Gene testing for NF1 and SPREDI was negative. CMMRD was suspected and a Lynch syndrome mismatch repair (MMR) gene panel was ordered. The panel testing revealed a pathogenic heterozygous c.1831dupA PMS2 mutation and a PMS2 exon 10 deletion, consistent with a diagnosis of CMMRD. Parental carrier testing is pending. This case highlights the importance of considering this newly described condition in the differential for CALs and pediatric cancer, particularly for those practitioners who evaluate children for NF1. Other NF1 stigmata (e.g. freckling, Lisch nodules, neurofibromas) have been described in CMMRD as well. In addition, family history from each side typically does not meet Amsterdam criteria for Lynch syndrome in the setting of CMMRD. Genetic counseling challenges include pre- and post-test counseling and follow-up not just for the affected child but also for the parents who are obligate carriers of a Lynch syndrome MMR gene mutation. New diagnostic criteria and surveillance recommendations have been published by international CMMRD consortiums giving preliminary direction for diagnosis and screening for these individuals.

Chromosome Microarray Results and Implications on Genetic Counseling: A Case Report Involving a 22q11.2 Distal Deletion

R. Mostafavi ${ }^{1}$, E. Pivnick ${ }^{1,2}$, A. Choudhri ${ }^{1,3}$, P. Klimo Jr. ${ }^{4,5,6,7}$,

1. Le Bonheur Children's Hospital

2. Department of Pediatrics, Division of Medical Genetics, University of Tennessee Health Science Center

3. Departments of Radiology, Ophthalmology, and Neurosurgery, University of Tennessee Health Science Center

4. Department of Neurosurgery, University of Tennessee Health Science Center

5. Le Bonheur Neuroscience Institute, Le Bonheur Children's Hospital

6. Semmes-Murpey Neurologic \& Spine Institute

7. Division of Neurosurgery, Department of Surgery, St. Jude Children's Hospital

Introduction: The American College of Medical Genetics and Genomics recommends chromosome microarray (CMA) for initial evaluation of patients with intellectual disability (ID), autism, or multiple anomalies. One under-addressed issue in pretest counseling of CMA is ability to diagnose conditions before complications present. We report a case of unexpected cancer predisposition diagnosed by CMA and discuss counseling issues. Case Report: A 16 month old male was referred to genetics for craniofacial anomalies. Family and medical histories were negative. Evaluation included CMA that revealed a $3.31 \mathrm{Mb} 22 \mathrm{q} 11.2$ distal deletion including SMARCB1, a tumor suppressor gene associated with increased risk for atypical teratoid/rhabdoid tumors (ATRT) in the brain and kidneys. Subsequent renal ultrasound showed no anomalies. MRI of the brain identified a highly cellular fourth ventricular mass that was circumscribed and showed no evidence of parenchymal invasion. The mass was surgically resected within $24 \mathrm{~h}$ of MRI and confirmed to be ATRT. After resection, patient was treated with chemotherapy and radiation and is disease-free 6 months later. The patient was evaluated by CMA for dysmorphic features. Parents did not expect results predisposing to cancer as this was not addressed in pretest counseling regarding CMA diagnostic yield in dysmorphic patients. Psychosocial implications for parents included distress and anxiety about management, recurrence risk, and prognosis. As no guidlelines exist to guide management of asymptomatic patients with SMARCB1 alterations, counseling of long-term patient care was limited. Discussion: CMA may be ordered on patients with SMARCB1 deletions due to dysmorphic features or delays evident before ATRT symptoms. Subsequent diagnosis of cancer predisposition carries significant psychosocial impact. Pretest CMA counseling for patients presenting with routine indications should address potential to diagnose asymptomatic conditions for which no cures or guidelines exist. Anticipatory guidance may reduce psychosocial burden of unexpected findings.

A Multidisciplinary Approach to Individualized Management of Cornelia De Lange Syndrome at the Children's Hospital of Philadelphia: The Parents' Perspective

\author{
S. Noon ${ }^{1}$, K. January ${ }^{2}$, K. Loomes ${ }^{1}$, A. Harrington ${ }^{1}$, M. Deardorff ${ }^{1}$, M. Pipan ${ }^{1}$, I. Krantz ${ }^{1}$ \\ 1. The Children's Hospital of Philadelphia \\ 2. Arcadia University
}

Cornelia de Lange Syndrome (CdLS) is a developmental diagnosis with variable growth, cognitive, craniofacial, structural and physiologic involvement of multiple systems. Many patients with CdLS need continued lifelong care due to the associated cognitive and behavioral impairment, physical limitations, and multi-systemic involvement. Given the clinical complexities and the need for multispecialty care, the Center for CdLS and Related Diagnoses was established at The Children's Hospital of Philadelphia (CHOP) to develop a comprehensive and integrated approach to clinical management and research issues relevant to CdLS. The Center's multidisciplinary clinic functions under the hypothesis that by training experts in relevant specialties to develop expertise in CdLS they will be able to proactively manage clinical issues and improve the outcomes and quality of life of our patients. The monthly clinic consists of four specialists and a consulting team of clinicians from divisions within CHOP. We work together, across specialties to develop an individualized plan of care for every patient. The clinic also serves as an interface with the Center's research goals to improve 
medical management and scientific understanding of CdLS that is critical for the translation of knowledge into improved diagnostics, management, and eventually therapeutics. Overall, our Center provides a setting in which patients can receive coordinated care, comprehensive services, family support, and the opportunity to participate in translational research. Little work has been done to evaluate the utility of a multispecialty approach to patient care. Here, we present the results from a survey of attitudes of parents of children with CdLS (those who have and have not attended a multispecialty clinic) attending the National CdLS Foundation family meeting. The objective is to better understand parental attitudes towards a multispecialty clinic approach and to determine the benefits and limitations of such a setting in caring for individuals with CdLS and complex developmental diagnoses in general.

\title{
Insulin Misregulation Underlies Circadian and Cognitive Deficits in a Drosophila Fragile X Model
}

B. Schoenfeld ${ }^{1}$, R. Monyak ${ }^{2}$, D. Emerson ${ }^{2}$, X. Zheng ${ }^{3}$, D. Chambers ${ }^{4}$, P. Hinchey ${ }^{5}$, C. Choi ${ }^{6}$, T. McDonald ${ }^{5}$, F. Bolduc ${ }^{4}$, A. Sehgal ${ }^{3}$, S. McBride ${ }^{7}$, T. Jongens ${ }^{2}$

1. Albert Einstein College of Medicine, University of Pennsylvania

2. Department of Genetics, Perelman School of Medicine, University of Pennsylvania

3. Department of Neuroscience and Howard Hughes Medical Institute, University of Pennsylvania

4. Department of Pediatric Neurology, Center for Neuroscience, INSTITUTION?

5. Molecular Cardiology Albert Einstein College of Medicine

6. Department of Dermatology, Drexel University College of Medicine

7. Department of Psychiatry, Perelman School of Medicine, University of Pennsylvania

Fragile X Syndrome is caused by loss of FMR 1 gene activity and is the most commonly inherited form of cognitive impairment and autism. Patients with this disorder also suffer from hyperactivity, attention deficit disorder, irritability, sleep problems and have noted neuro-anatomical defects. A Drosophila fragile X model, based on loss of $d f m r l$ function, displays several relevant phenotypes, including defects in circadian regulation, social interaction (with peers and in naïve courtship), memory and morphology of some neurons. Here we show that the circadian and memory deficits displayed by $d f m r l$ mutants are due to enhanced insulin signaling. In a study aimed at mapping the spatial requirements of $d f m r l$ activity for normal circadian regulation, we found that select expression of $d f m r l$ in the insulin producing cells (IPCs) of the brain is sufficient to restore normal circadian behavior. Surprisingly, this select expression also rescues the memory deficits in the fragile X model. Examination of the insulin pathway reveals elevated levels of Drosophila insulin-like peptide 2 (Dilp2) in the IPCs and elevated insulin signaling throughout the $d f m r 1$ mutant brain. Consistent with elevated insulin signaling being causal in $d f m r l$ mutant phenotypes, we found that select expression of $d f m r l$ in the IPCs reduces insulin signaling and that genetic reduction of the insulin pathway also leads to amelioration of these phenotypes. Finally we show that treatment with the FDA approved drug metformin can also rescue the memory in two different paradigms. Our results indicate that insulin misregulation underlies the circadian and cognitive phenotypes displayed by the Drosophila fragile $\mathrm{X}$ model, and thus reveals another pathway that can be targeted by new and current drugs to treat fragile $\mathrm{X}$ patients.

\section{Further Evidence of a Milder Phenotype Associated with the V80F and D132A Mutations in EXOSC3}

\section{A. Schreiber ${ }^{1}$, T. Moss ${ }^{1}$}

\section{Cleveland Clinic}

Our proband was a 3-year-old male who presented with gross motor delays, ataxia, tremor, and poor expressive speech. He began cruising at 18 months and was able to take a few independent steps at 2.5 years, although he primarily used a walker or forearm crutches for ambulation. He had an appropriate vocabulary but his speech was dysarthric. His brain MRI revealed cerebellar hypoplasia and abnormal myelination. No dysmorphic features were noted and his family history was negative for similarly affected relatives. Previous genetic testing, including chromosome oligoarray, Fragile X, the POLR $3 \mathrm{~A}$ and $P O L R 3 B$ genes, and transferrin with N-glycan were normal. Clinical whole exome sequencing (WES) was obtained and identified compound heterozygous mutations in the EXOSC3 gene, which is associated with pontocerebellar hypoplasia type 1B (PCH1B). This is an autosomal recessive condition characterized by diffuse muscle weakness, hypoplasia or atrophy of the cerebellum and pons, progressive microcephaly, very little motor development, respiratory failure, and death often in the first year of life. We present the second report of a patient with a much milder phenotype associated with a particular genotype. The patient's mutations, V80F and D132A, have been reported previously in one family with a similar milder clinical course. These siblings were adolescents with mild to moderate intellectual disability, spasticity, cerebellar atrophy, and dysarthria. The D132A mutation has also been reported in a homozygous state in patients with some motor development and survival at least into early adulthood.

Our case provides additional evidence that the D132A mutation in EXOSC3 is associated with a less severe phenotype of PCH1B, and that when found in combination with the V80F mutation does not cause the microcephaly, profound motor impairment, and poor outcome typical of this condition. This may help define the mild end of the clinical spectrum for patients with PCH1B.

Comparing Face Perception Skill among Individuals with Williams Syndrome, Autism Spectrum Disorder, Prader-Willi Syndrome, Mild Intellectual Disability and Typical Development

\author{
K. Schultz ${ }^{1}$, J. Wolf ${ }^{2}$, C. Klaiman ${ }^{3}$, J. Herrington ${ }^{4}$, K. Valverde ${ }^{1}$, B. Pober ${ }^{5}$, R. Schultz ${ }^{4}$ \\ 1. Arcadia University \\ 2. Yale University \\ 3. Emory University School of Medicine, Marcus Autism Center \\ 4. University of Pennsylvania, Children's Hospital of Philadelphia \\ 5. Harvard Medical School, Boston Children's Hospital
}


Social communication is affected in many genetic forms of developmental disability, with a deleterious impact on quality of life. Face perception for identity and memory, and for inferring another's feelings and intentions is important for effective social communication. Existing literature on this topic in developmental disabilities, including Autism Spectrum Disorder (ASD) and Williams syndrome (WS) offers mixed results. To clarify this issue we compared face identity perception among individuals with ASD and WS to three comparison groups - typically developing controls (TDC), Prader-Willi syndrome (PWS), and mild intellectual disability (ID) using the Benton Facial Recognition Test and standardized IQ tests. A total of 544 participants were assessed, including 39 with WS and 185 with ASD; mean age was early teens. We hypothesized that TDCs would perform the best, followed by WS, ID, PWS and ASD. An analysis of variance found significant differences between the groups $(\mathrm{F}[4,544]=53.90, p<.001)$, with TDCs outperforming all other groups, as expected. However, face recognition ability was significantly correlated with IQ $(\mathrm{r}=.48, p<.001)$, which differed between the groups (e.g. WS IQ was more than 40 points lower than TDCs). An ANCOVA, controlling for IQ, found that the WS group performed equivalently to the TDCs $(\mathrm{F}[1,267]=.41, p=.52)$, and both were better than the other three groups (p's<.01). The ASD, PWS and ID groups did not differ from each other (p's>.15). These results have important implications for the neurobiology of developmental disorders, for phenotype/genotype correlations, as well as important clinical implications. Future work to develop face recognition interventions should consider the impact of general cognitive impairment in successful social adaption.

\section{Prevalence of Genetic Conditions and Developmental Delays in Emergency Department Visits for Poison and Foreign Body Ingestions}

\section{E. Sengstock ${ }^{1}$, R. Stewart ${ }^{2}$, S. Nagappan ${ }^{3}$, P. Reitnauer ${ }^{3}$}

\section{Essentia Health \\ 2. University of North Carolina at Greensboro \\ 3. Moses Cone Hospital}

Background: McCandless et al. (2004) estimated that children with underlying genetic conditions make up $71 \%$ of hospital admissions. The primary acute diagnoses on admission for children with underlying genetic conditions has not been adequately studied to date, although common reasons for pediatric hospital admissions include poison ingestion, foreign body ingestion, and adverse drug events. Per Sherrard et al. (2002), children with underlying conditions may have features that predispose them to ingestions and thus morbidity, which is preventable through education and supervision. Purpose: The goal of this study was to determine the prevalence of children with genetic conditions or developmental delays among children seen in the emergency department for ingestions including poisons, medications, and foreign bodies. Additionally, this study examined some of the demographic factors that potentially provide a poison ingestion risk. Methods: A retrospective medical record review was conducted using records of children ages 6 months to 18 years with ICD-9 diagnoses in the ingestion categories from Cone Health in Greensboro, NC from January 1, 2010 through January 31, 2012. Data regarding the type of ingestion, underlying condition, and other demographics was abstracted and analyzed. Results: Children with underlying genetic conditions comprised $20 \%$ of children seen for ingestions during this time period $(p<0.05)$ and had an average age that was 6 years older at the time of ingestion compared to children without genetic conditions $(p<0.05)$. The prevalence of children with single gene/chromosomal conditions was $1.2 \%$ which was higher than the estimated population prevalence of $0.5 \%$ by the age of 25 , suggesting that there is a higher risk of ingestion in this population. The most common source of ingestion was medication for children with a genetic condition and foreign bodies for those without. Conclusion: Genetic counselors can provide anticipatory guidance to parents of children with a genetic condition regarding this risk of ingestion to prevent morbidity.

Disclosure of a Fragile X Syndrome Diagnosis to Symptomatic Females: A Qualitative Study of the Parental Approach

L. Stobie ${ }^{1}$, A. McConkie-Rosell ${ }^{2}$, S. Lincoln ${ }^{3}$, D. Devine ${ }^{4}$, L. Mandel ${ }^{1,5}$, G. Schneider ${ }^{1}$

1. Brandeis University

2. Duke University School of Medicine

3. Boston Children's Hospital

4. National Fragile X Foundation

5. Regis College

Discussing the implications of a genetic condition with an affected child is challenging; it may be even more so in conditions such as fragile X syndrome (FXS) when the child is cognitively impaired and at risk of passing the same condition to offspring. To date there have been no studies focused on the disclosure of a FXS diagnosis to symptomatic girls with FMR1 full mutations. The purpose of this study was to explore what, how, and when parents disclosed the diagnosis of FXS to their symptomatic daughters. We conducted semistructured interviews with six mothers recruited through the National Fragile X Foundation, FRAXA Research Foundation, and Facebook. We coded and analyzed the interview transcripts using grounded theory and Atlas.ti software. The mothers' ages ranged from 43 to 54 years. The daughters ranged in age, from 3 to 14 years at diagnosis, and 14 to 26 years at the time of this study. The average age of the daughters at the time of disclosure was 13 years. In most instances, the disclosure took place years after the initial diagnosis. The mothers we interviewed reported a preference for open communication and tried to give their daughters a positive outlook when discussing FXS with them. We found that most mothers used their daughters' learning difficulties to initially explain their FXS diagnosis, and some also used comparison to a male relative as a tool for explanation. The majority did not focus their conversations on the reproductive risks associated with FXS. The parental challenges that emerged included guilt over carrier status, difficulty determining if daughters understood the information, and lack of guidance from health care professionals regarding disclosure. Future studies should focus on daughters' perspectives of the disclosure process to provide additional insight regarding how to most effectively communicate this information. 


\title{
FBN1 Mutation Identified in Critically Ill Neonate with a Diagnosis of Marfan Syndrome
}

\author{
J. Tarpinian' ${ }^{1}$, M. Knepp ${ }^{2}$, J. Flores ${ }^{3}$ \\ 1. Division of Pediatric Genetics, University of Illinois College of Medicine \\ 2. Division of Pediatric Cardiology, University of Illinois College of Medicine at Peoria \\ 3. Division of Medical Genetics, University of Illinois College of Medicine at Peoria
}

Marfan syndrome (MFS) is an autosomal dominant connective tissue disorder characterized by cardiovascular, ocular, and skeletal anomalies and is caused by mutations in the FBN1 gene. Clinical severity and age of onset is variable. The well-established Ghent criteria were established to aid in the clinical diagnosis and identification of an FBN1 mutation. The most severe form, neonatal MFS, presents with atrioventricular valve dysfunction, pulmonary emphysema, and joint contractures; however, diagnostic criteria is debated. Patient was born at 36 3/7 weeks gestation to a G3P1 mother via cesarean section due to fetal cardiac failure. Pregnancy was complicated by late-term cardiac dysfunction and oligohydramnios. Patient was cyanotic at birth and transferred to Neonatal ICU. Echocardiogram revealed mitral and tricuspid valve insufficiency, biventricular failure, and aortic root dilation (Zscore=3.73). Physical exam revealed long slender phalanges, underdeveloped ears, and non-characteristic facial dysmorphisms. Extracorporeal membrane oxygenation was initiated. Clinical course was complicated by abdominal compartment syndrome secondary to a massive liver hematoma. At day of life 7, patient was in a state of multi-organ failure without improvement. Care was withdrawn. Karyotype and chromosomal microarray analysis revealed normal female chromosomal complement. FBN1 sequencing analysis revealed a disease-causing mutation. We present a case of a critical neonate with severe mitral and tricuspid insufficiency, cardiomyopathy, and multi-organ failure identified to have a heterozygous c.3964+1 G $>$ A mutation in the FBN1 gene, consistent with MFS. Ghent criteria were not applicable in this patient presentation. Stheneur et al. (2011) showed arachnodactyly, ascending aortic dilation, facial dysmorphism, flexion contractures, and valvular insufficiencies were the most commonly present findings in probands affected with MFS at less than 1 year of age. Our patient's clinical findings are supportive of this evidence and help establish diagnostic criteria for early onset MFS

Death of a Child to Tay-Sachs and Other Progressive Neurological Disorders: Long-Term Impact on Parents' Emotional and Personal Lives

\author{
E. Williams ${ }^{1}$, D. Obler $^{2}$, B. Lord ${ }^{3}$, T. Cross ${ }^{1,4}{ }^{4}$. Tsipis ${ }^{1}$ \\ 1. Brandeis University \\ 2. Genetic Consultation \& Counseling - Private Practice \\ 3. National Tay-Sachs \& Allied Diseases Association \\ 4. Cross Associates
}

Though studies have explored the intense emotions parents may experience shortly after the death of their child, including anger, denial, and guilt, few have examined the lasting effects of this loss. The purpose of this study was to identify the long-term impacts on parents after the death of their child due to a rare, genetic disease that is incurable, neurologically progressive, and life-limiting. We recruited the parents of children who died two or more years ago through the National Tay-Sachs \& Allied Diseases Association and the National Mucopolysaccharidoses Society via email sent to approximately 285 families. Participants $(n=62)$ completed an online, anonymous survey that included questions about the deceased child's illness and death, changes in their relationships with family, friends, work and faith as well as validated assessments of prolonged grief and personal growth following a traumatic experience. Data was evaluated by frequency, descriptive statistics, and correlation analysis. Participants, on average, experienced a moderate amount of growth, with highest mean scores in the categories of "appreciation of life" and "personal strength". Some parents reported difficulty motivating themselves for certain activities and trouble connecting with people who had not experienced a similar loss, but only a small minority $(6.7 \%)$ met criteria for prolonged grief disorder. Since the death of their child, parents reported improvement in their relationships with their other children but little change in their relationships with their parents, siblings, friends, colleagues and their work. The largest proportions of change, both positive and negative, were reported in the close personal relationships with their partner or child's other parent, as well as their spiritual faith. These results highlight bereaved parents' coinciding experiences of anguish and positive growth following their child's death. Using this information, healthcare professionals may help families affected by life-limiting conditions to understand their future emotional evolution.

Expanding the Phenotype of Mitochondrial Disease: What Every Genetic Counselor Needs to Know

S. Wong' ${ }^{1}$ L. Hasadsri ${ }^{2}$, C. Balcells ${ }^{3}$, C. Stanley ${ }^{1}$

1. Courtagen Life Sciences

2. Mayo Clinic

3. MitoAction

Mitochondrial disease has typically been known as primarily a disorder of neuromuscular manifestations, brain abnormalities, or various syndromes such as MELAS, MERFF, and MNGIE. However, mitochondrial disease is an extremely pleiomorphic condition which can present in almost any system of the body. Recent controversies in the news, such as the plight of Justina Pelletier who is reportedly diagnosed with mitochondrial disease by one physician but claimed to have a somatoform (psychiatric) disorder by others, have highlighted the lack of awareness and understanding of both the medical community and the general public regarding the non-classical or lesser known phenotypic features of mitochondrial disorders, and underscored the need for education in this aspect. Particular complicating factors that will be addressed include: 1) Manifestations may be episodic (come and go), even lab abnormalities such as serum lactate or organic acids. 2) Symptoms can be extremely subjective, such as significant pain, nausea, and neuropathy, and are often not measurable by ancillary studies or laboratory analyses, but only by patient report. Due to their subjective nature, these symptoms are often perceived as made up by patients or parents, when in fact they can be debilitating and disabling to the patient. 3) Symptoms that are common in mitochondrial disease but are not well known to many genetics professionals obtaining medical histories and pedigrees. As these conditions are 
extremely common in the general population they may not be recognized as mitochondrial symptoms in the absence of the right context. Examples of these symptoms are autonomic nervous symptom dysfunction (dizziness, POTS), gastrointestinal disease (IBS, GERD), pain disorders (complex regional pain, migraines), chronic fatigue, and psychological conditions (anxiety, depression, panic). With knowledge of the expanded phenotype of mitochondrial disease, genetics professionals can play an important role in guiding more appropriate diagnoses and educating fellow medical professionals and the general public.

\title{
Prenatal
}

The Down Syndrome Prenatal Diagnosis Study: Understanding the Informational and Emotional Needs of Individuals with a Prenatal Diagnosis of Down Syndrome

\author{
K. Berrier Sheets ${ }^{1}$, B. Crissman ${ }^{1}$
}

\section{Duke University Medical Center}

Prenatal diagnosis literature is largely limited to retrospective perceptions of patients who either continued or terminated pregnancies after a prenatal diagnosis of Down syndrome (DS). Increased uptake of noninvasive prenatal testing (NIPT) underscores the need for further investigation of the prenatal diagnosis experience and how healthcare providers can best support our patients. This two-year exploratory study aims to identify informational and emotional needs of patients during the decision-making process for pregnancy management. Participants voluntarily access an anonymous web-based REDCap questionnaire collecting information about the pregnancy, diagnosis, genetic counseling process and influential factors in reproductive decision-making. The convenience sample includes men and women who received a prenatal diagnosis of DS by amniocentesis, chorionic villus sampling (CVS) or NIPT in an ongoing or recent pregnancy. Results from year one (1 JAN 2013-31 DEC 2013) include $n=77$ respondents from 32 states, Canada and Germany who represent our target sample of patients with a prenatal diagnosis of DS in 2013 ( $n=28$ by amniocentesis, 36 NIPT, 12 CVS, 1 unsure). Most had made a final decision by the time they completed the questionnaire ( $n=7$ termination, 1 adoption plan, 33 parent the child, 11 no response); while two were taking steps toward implementing their decision ( $n=1$ termination; $n=1$ parent the child) and two were still considering available options. A post-test was completed by 124 patients who rated the research experience: $99 \%$ felt that their participation could potentially help others and $97 \%$ found it beneficial. While few found participation somewhat stressful, most did not. No one rated the overall research experience as negative, suggesting that prenatal diagnosis patients are not too vulnerable to participate in research. Insightful viewpoints from patients serve as a timely appraisal to inform evidence-based practice and help us better support patients receiving and making various decisions for a prenatal diagnosis of DS.

\section{Prenatal Indicators of Sotos Syndrome}

\author{
S. Burnett ${ }^{1}$, I. Abumansour ${ }^{1}$, P. Frosk ${ }^{1}$, B. Chodirker ${ }^{1}$ \\ 1. Genetics and Metabolism Program, Winnipeg Regional Health Authority
}

\section{Introduction}

Sotos syndrome is an autosomal dominant condition with characteristic facial dysmorphism, learning disability and childhood overgrowth caused by mutations in the NSD1 gene. There is a paucity of information regarding prenatal presentations in Sotos syndrome. Literature review uncovered two reports with a history of a positive maternal serum screen (MSS). In addition, there was evidence of abnormal sonographic findings and/or pre-eclampsia in some cases. Here we present a patient with a prenatal diagnosis of Sotos syndrome and two additional patients identified through retrospective review of our genetics database that had prenatal findings as well. Case 1: A 26 year old G2P1 presented with a positive MSS result indicating a $>=1: 8$ risk for Down syndrome. Later in gestation an Ebstein's anomaly and pleural effusions were identified on ultrasound and the pregnancy became complicated by maternal hypertension. Intrauterine death occurred at 29 weeks. Microarray analysis indicated a heterozygous deletion of NSD1, consistent with a diagnosis of Sotos syndrome. Case 2: A 12 year old boy presenting with developmental delay received a clinical diagnosis of Sotos syndrome. Microarray identified a heterozygous deletion of NSD1. Records revealed the boy's mother had a positive MSS result with a 1:30 risk for Down syndrome. Case 3: A 9 year old boy presented with developmental delay and dysmorphic facial features. The patient met the criteria for a clinical diagnosis of Sotos syndrome. Molecular analysis of NSD1 was not done. Maternal hypertension and a positive MSS with a Down syndrome risk of 1:127 complicated the pregnancy. Discussion: Our report demonstrates that Sotos syndrome can be diagnosed prenatally in some cases and should be included in the differential diagnosis for a false positive maternal serum screen for Down syndrome, particularly if ultrasound abnormalities and/or maternal obstetrical complications are present.

\section{Attitudes of Prenatal Genetic Counselors regarding Pan Ethnic Carrier Screening and Development of a Pre-Counseling Informational Mobile Webpage}

\author{
K. Curd ${ }^{1}$, T. Causey ${ }^{2}$ \\ 1. Savannah Perinatology Associates, University Medical Center \\ 2. Virginia Commonwealth University Health System
}

In recent years, technology has expanded such that it is now possible to simultaneously screen individuals for carrier status of numerous different genetic diseases. Expanded pan ethnic carrier screening (EPECS) is a screening test that has been developed as a cost effective method capable of analyzing greater than 100 diseases concurrently. With EPECS being offered more regularly, it is important to address the benefits of testing and concerns healthcare professionals have identified when it comes to implementation. Opinions of healthcare professionals are mixed when it comes to implementation of EPECS, with many stating that expanded carrier screening is, and should be, the standard of care and others stating that expanded carrier screening is not ready for routine use. The current study seeks to identify how genetic counselors are using EPECS in their current practice and 
what they perceive to be benefits and concerns associated with EPECS. Results showed that genetic counselors agree with the literature in regards to benefits and concerns and are choosing to offer EPECS to patients that ask for additional testing, preconception patients, and consanguineous couples. Genetic counselors also identified what they felt should typically be covered in a session and what they felt were the most important aspects of EPECS. This Information was then used to create a mobile webpage that can be used by patients in a pre-counseling setting.

Not the Answer we Expected: Detection of Fetal 47,XXX via Noninvasive Prenatal Testing in the Setting of Increased Nuchal Translucency M. Discenza ${ }^{1}$, E. Thorpe ${ }^{1}$, F. R. Bieber ${ }^{1}$, R. E. Reiss ${ }^{1}$, L. E. Wilkins-Haug ${ }^{1}$

\title{
1. Brigham and Women's Hospital
}

Introduction: Measurement of nuchal translucency (NT) in first trimester is a well-established screening tool for trisomies 21, 18, 13, and monosomy X. However NT may be increased in other aneuploidies whose clinical significance is less clear. We report 2 confirmed cases of 47 , XXX where patients initially deferred diagnostic testing in favor of noninvasive prenatal testing (NIPT). The potential variability in both fetal and phenotypic presentation of 47,XXX may leave many cases undiagnosed and presents dilemmas for genetic counseling. Case Descriptions: Patient 1 was a 35 y.o. G4P1, seen for NT evaluation at 11.7 weeks of gestation. NT measured $4.9 \mathrm{~mm}$ (normal 0-2.4 mm). NIPT predicted 47, XXX. Evaluation at 13.7 weeks revealed persistent lateral neck fluid collections consistent with cystic hygroma. Invasive testing was declined. Fetal survey at 15.7 weeks showed an echogenic intracardiac focus (EIF) and prominent left renal pelvis. Cystic hygroma had resolved. Fetal survey at 19.3 weeks showed an EIF and normal nuchal fold. Karyotype on amniocytes confirmed 47, XXX. Fetal echocardiogram, oligo microarray and Noonan syndrome testing were normal. At delivery, cord blood karyotype was 47,XXX. Patient 2 was a 32 y.o. G3P1SAB1 seen at 11.1 weeks. NT measured $3.6 \mathrm{~mm}$ (normal 0-2.4 mm). NIPT predicted 47, XXX and maternal karyotype was normal (46,XX). Fetal karyotype on amniocytes confirmed 47,XXX. Fetal survey, echocardiogram and oligo microarray were unremarkable. Discussion: Though Sebire et al. (1998) reported 47,XXX among their population with increased NT; the association was weaker than for autosomal trisomies. Thus confirmation of NIPT with diagnostic testing is important to exclude a maternal sex chromosome aneuploidy or mosaicism, and to ensure that no other chromosomal abnormality is present as a cause of a large NT. As NIPT expands beyond aneuploidy detection, the risk for incidental findings will increase. Our cases stress the importance of pre-test counseling for NIPT to underscore its limitations and the possibility of findings with uncertain clinical significance.

\section{An Evaluation of Referral Indications for Prenatal Genetic Counseling over a 15 Year Period}

\section{Durand ${ }^{1}$, D. Ramsey ${ }^{1}$}

\section{Integrated Genetics}

This study examines referral indications for prenatal genetic counseling patients and the observed shifts over the past 15 years. Data was collected on 896,295 nationwide referrals for prenatal genetic counseling at Integrated Genetics from 1999 through 2013. The indications were divided into 8 categories: advanced maternal age (AMA), abnormal serum screening (MMS), family history, exposures, abnormal ultrasound, California Prenatal Screening and other. The other category is most commonly comprised of average risk patients referred to discuss aneuploidy screening and diagnostic procedure options available to them. The most significant changes from 1999 to 2013 were seen in the following categories: (a) MMS: decrease from 14.10 to $8.01 \%$ ( $p<0.0001)$; (b) AMA: decrease from 51.12 to $46.42 \%$ ( $p<0.0001)$; (c) abnormal ultrasound: increase from 4.88 to $7.44 \%(p<0.0001)$; (d) family history: increase from 11.19 to $17.58 \%(p<0.0001)$; and (e) other: increase from 4 to $6.56 \%(p<0.0001)$. Referrals for advanced maternal age remain the bulk of referrals for genetic counseling. However, the percentage of referrals for AMA has decreased, with the greatest decrease observed in the last 5 years. This decrease may be related to changes in obstetrical practice patterns involving a shift from referring patients based on maternal age to referring patients based on fetal risk. This paradigm shift was supported by the 2007 American College of Obstetricians and Gynecologists practice bulletin recommending that a two-tiered aneuploidy screen be offered to all pregnant women regardless of age. As aneuploidy screening has improved with decreased false positives, fewer women are being referred for abnormal screens. Given the increasing complexity of screening options, we have seen a significant increase in the "other" category, as physicians may be less comfortable counseling about the available aneuploidy screening options. As the referral indications for prenatal genetic counseling continue to shift with the changes in technology, genetic counselors will need to adapt to seeing a higher percentage of patients with more complex indications

\section{Walking the Edge with Controversial Use of Preimplantation Genetic Diagnosis: Opinions and Attitudes of Genetic Counselors}

\author{
K. Everton ${ }^{1}$, J. Edwards ${ }^{1}$, A. Besser ${ }^{2}$, P. Hershberger ${ }^{3}$ \\ 1. University of South Carolina \\ 2. Bonei Olam \\ 3. University of Illinois at Chicago
}

Introduction: We explored opinions and attitudes of genetic counselors regarding three controversial applications of preimplantation genetic diagnosis (PGD): PGD for early-onset Alzheimer, use of embryos that are BRCA positive after PGD revealed no disease-free embryos to be available, and PGD to select against a variant of unknown significance (VUS) for Marfan syndrome. Methods: Inclusion criteria required that a participant was currently practicing as a genetic counselor, a member of the NSGC, and counseled patients about PGD. Twenty-four recorded interviews were transcribed for data analysis. The survey consisted of demographic questions, qualitative questions about each of the three case scenarios, and general questions about PGD. Qualitative analysis was performed using a conventional content analysis approach. Results: Themes common to all three scenarios included: necessity of appropriate/thorough counseling, the importance of the genetic indications, patient perceptions, and respect for patient autonomy in decision making. Multiple themes were also described for each unique case scenario. Fifty-eight percent (14/24) had little to no concerns about PGD for an adult-onset 
disorder. Eighty-eight percent (21/25) had concerns about PGD for a VUS. The majority (65\%,15/23) of participants felt PGD for an adult-onset disorder was least controversial, and PGD for a VUS was most controversial. Conclusion: Participants felt that PGD was appropriate for life limiting conditions, cases where there was an established diagnosis with a known pathogenic mutation, and when symptom severity and disease burden were significant. Participants agreed that appropriate/thorough counseling was necessary, patient perceptions of 'serious' disease were critical, and patient autonomy were key factors when dealing with controversial applications of PGD. Genetic counseling is recommended. Patients need to understand the benefits, disadvantages, and potential outcomes of PGD in order to make the decision that is most appropriate for their families.

\title{
Recurrent Bilateral Renal Agenesis: Expanding the Spectrum of Fraser Syndrome
}

M. Ferguson" ${ }^{1}$, N. Blagowidow ${ }^{1}$

\section{Greater Baltimore Medical Center}

Introduction: Bilateral renal agenesis (BRA) is a rare finding with an incidence on fetal autopsy of $1 / 240$. The etiology is heterogenous, but often undetermined. We present a couple with their third affected pregnancy. Case: The couple had four pregnancies in a 3 year period. One resulted in spontaneous abortion. The others were found to have BRA on 2nd trimester ultrasounds. An amniocentesis was performed on the third affected pregnancy. Cytogenetic studies were performed on the second and third pregnancies. Chromosomes were normal but microarray revealed regions of homozygosity, suggesting consanguinity. The couple revealed that they were consanguinious. Areas of homozygosity were reviewed for genes associated with renal agenesis and a gene associated with Fraser syndrome, FRAS1, on 4q21 was identified as a candidate gene. The couple was offered testing for FRAS1 gene versus whole exome sequencing (WES) and opted for the latter. WES studies revealed homozygous gene mutation (c.2570 G>T) in the FRAS1 gene, confirmed in 2 of the pregnancies. Discussion: The etiology of BRA is variable including a familial association with unilateral renal agenesis, chromosomal abnormalities, single gene disorders, and teratogenisis. Couples who experience a pregnancy with BRA are often provided with a wide range of recurrence risks from 3.5 to $25 \%$. Features of Fraser syndrome (FS) include cryptophthalmos and other eye defects, limb anomalies, genital abnormalities. Renal hypoplasia or agenesis is reported in $37 \%$. Other findings include intellectual disability and brain and facial abnormalities. In our patient's pregnancies no other features consistent with FS were observed on ultrasound or fetal evaluation. These pregnancies presented only with BRA. This is the second recent report of isolated renal anomalies associated with Fraser syndrome gene mutations. WES proved to be a useful strategy in defining an etiology for this family and confirms an expansion of the spectrum of FS.

Termination Rates and Influencing Variables for Pregnancies Affected by Aneuploidy

K. Garfield ${ }^{1}$, A. Mathiesen ${ }^{1}$, J. Byrne ${ }^{1}$, D. Turok ${ }^{1}$, K. Charyk ${ }^{2}$, J. Sorensen ${ }^{1}$

1. University of Utah

2. Intermountain Healthcare

Introduction: We sought to identify factors associated with the decision to terminate a pregnancy affected with aneuploidy among a conservative religious population. Additionally, we compared the termination rate for trisomy 21-affected pregnancies with previously reported rates. Hypotheses: We hypothesized that certain maternal social factors and pregnancy characteristics are associated with the decision to terminate pregnancies diagnosed with aneuploidy. We also hypothesized that termination rates for pregnancies affected with trisomy 21 are lower in Utah than the literature suggests. Methods: Data were obtained through retrospective record review of 164 pregnancies diagnosed with aneuploidy from 2007-2011 at University Health Care and Intermountain Healthcare clinics in Utah. We examined maternal age, race, relationship status, maternal insurance status, gestational age, fetal diagnosis and gender, ultrasound findings and parity. We also evaluated individuals' proximity to an abortion provider. Data was analyzed using chi-square tests, ttests, and multiple logistic regression. Results: Controlling for other variables, pregnancies affected with aneuploidy were significantly less likely to be terminated when structural anomalies were visualized on ultrasound. Lethal aneuploidies and those with trisomy 21 were significantly more likely to be terminated. Likelihood to terminate was inversely associated with distance to an abortion provider. Trisomy 21 termination rates were lower than those previously reported among other populations. Other factors were not significantly associated with termination. Conclusions: Our data suggest that the choice to terminate a pregnancy affected with aneuploidy is complex, even among women from a conservative culture. Additionally, distance to abortion providers can be a barrier to accessing such services when a pregnancy is diagnosed with aneuploidy.

\section{The Paternal Age Effect: A Preliminary Study of Current Challenges for Prenatal Care}

\author{
A. Gunter ${ }^{1}$, M. Walker ${ }^{1}$, I. Lipkus ${ }^{2}$, H. Toriello ${ }^{3}$ \\ 1. University of South Carolina \\ 2. Duke University \\ 3. Spectrum Health
}

Introduction: Recent literature has expanded our understanding about the causes of advanced paternal age (APA) risks for various genetic conditions, behavioral disorders, and adverse pregnancy outcomes. However, no guidelines exist to refer APA pregnancies to prenatal genetics care, nor are effective screening techniques presently available. The objective of this study was to determine if updates in research and preferences of prenatal patients warrant a reassessment of APA pregnancy care. Methods: Surveys about advanced maternal age (AMA) and APA implications were offered to patients and their partners at the end of prenatal genetic counseling sessions. Fifty-eight individuals (AMA mothers and APA fathers $>39$ years of age) were invited to participate over a four-month period at a prenatal clinic in South Carolina. Participants were asked about their understanding of age-related risks to pregnancy, preferences regarding genetics education, and favorability toward potential future screening using Likert scale, knowledge, and opinion questions. Statistical analyses included Fisher exact test for dependency, Spearman's rho for correlations, and percentages for frequency analysis based on demographic information. Short responses to open-ended questions were qualitatively analyzed using Grounded Theory methods. Results: Over half 
of the 23 respondents ( $57 \%$ ) indicated understanding of increased risk for autism in children of older fathers, but not for Down syndrome ( $35 \%$ ). Seventy percent favored receiving education on all risks associated with parental age. Seventy-four percent favored future screening for risks for autism, $61 \%$ for seizure disorders, and $54 \%$ for learning disabilities. Conclusion: Based on our study findings and support in the literature, we support a collaborative effort to address the shortcomings of current prenatal genetic counseling about the risks of APA. We advocate for revisiting the 2008 American College of Medical Genetics and Genomics guidelines concerning APA and for directing research efforts toward the future goal of providing inclusive genetic counseling for men of APA and the pregnancies they father.

\title{
Conception through Gamete Donation: Challenges in Genetic Testing and Counseling of Gamete Donor Recipients
}

L. Isley ${ }^{1}$, P. Callum ${ }^{1}$

\section{California Cryobank}

Gamete donation is a common method of assisted reproduction leading to thousands of births every year. Genetic counselors (GCs) who work in reproductive genetics frequently encounter patients who are planning a pregnancy or who have conceived through gamete donation. There are unique counseling issues with which genetic counselors need to be familiar when working with these populations, particularly when offering patients expanded carrier screening or prenatal chromosomal microarray analyses (CMA). Specifically: (1) Genetic screening on gamete donors is variable and is more likely to be limited to American College of Medical Genetics and Genomics or American College of Obstetricians and Gynecologists recommended tests than expanded carrier screening panels; (2) When offering expanded carrier screening panels to a patient, it is important to discuss that anonymous gamete donors may not be available to participate in additional genetic screening should the recipient have a positive test result; (3) The donor may not provide consent to participate in testing; (4) In some cases it can take several months to coordinate testing on the donor and obtain results, which can be concerning if pregnancy management decisions are dependent on the donor's result; and (5) The interpretation of a variant of unknown significance may be complex when many healthy offspring exist from that donor's specimens. Genetic counselors were surveyed at the 2013 NSGC Annual Education Conference regarding their knowledge of gamete donor screening practices. Only $10 \%$ of those GCs who work in prenatal and/or assisted reproductive technology settings reported being very familiar with the gamete donor screening recommendations (unpublished data). Twenty-nine percent reported that they do not have any knowledge of these practices. It is advisable that reproductive counselors pursue education in these issues or collaborate with genetic counselors within the gamete donor industry in order to facilitate positive genetic testing experiences for their patients.

\section{Genetic Evaluation of Products of Conception and Deceased Fetuses after Miscarriage and in Cases with Abnormal Ultrasound Findings:} Experiences of a Diagnostic Laboratory

\author{
A. Janze ${ }^{1}$, E. Kramer Dugan ${ }^{1}$, G. Richard ${ }^{1}$, E. H. Andrew ${ }^{1}$, L. Matyakhina ${ }^{1}$, J. Meck ${ }^{1}$
}

\section{GeneDx}

It is important for genetic counselors to be aware of available genetic testing options for products of conception or deceased fetuses and how these results can affect patient decisions and overall patient satisfaction. We reviewed results from over 250 fetal specimens with variable indications for genetic testing, including investigating the cause of miscarriage/intrauterine fetal death and multiple anomalies. There were 105 cases (42\%) with an abnormal result. Forty-three (41\%) of those were aneuploidies, including monosomy X, trisomies 21, 13, 18, 22, 15, 16 and other less common chromosomes (4, $6,7,8,10,14)$, as well as two cases of double aneuploidy. There were 11 polyploid cases. Additionally, 27 fetuses $(26 \%)$ had other chromosomal anomalies by array, including recurrent microdeletion syndromes (e.g., RCAD, TAR, 3p-). Microarray yielded results on six of 12 cases where culture failure precluded chromosome analysis. Three of these were positive, and patients were able to get clinically useful information. Gene panels or single gene tests should be considered when there are fetal anomalies consistent with a particular phenotype. Examples are holoprosencephaly, X-linked hydrocephalus, Noonan syndrome, Smith-Lemli-Opitz syndrome, Joubert syndrome, ectrodactyly, campomelic dysplasia and microphthalmia. Twentyfour cases (23\%) had a gene-specific anomaly, including mutations in L1CAM, DHCR7, SOX9, KRAS, PTPN11, TP63, ABCA12, SHH and CC2D2A. For ultrasound findings that do not point to a particular disorder, whole genome array and/or whole exome sequencing may be considered. Our data demonstrate a high likelihood of identifying a causative genetic abnormality in this cohort and underscore the value of genetic testing for providing families with an understanding of future reproductive risks. The finding of aneuploidy or other non-hereditary abnormality can help families find acceptance and closure, while the identification of a hereditary condition can facilitate future preconception care and testing for family members.

\section{Prenatal Diagnosis of Pallister Hall Syndrome: A Case Report}

\author{
S. Kasperski ${ }^{1}$, S. Chadwick ${ }^{1}$, E. Zackai ${ }^{1}$, B. Coleman ${ }^{1}$, R. Zimmerman ${ }^{1}$, E. Wang ${ }^{2}$, J. Moldenhauer ${ }^{1}$ \\ 1. Children's Hospital Of Philadelphia \\ 2. Hospital of the University of Pennsylvania
}

Improved prenatal imaging modalities, including 3D ultrasound and fetal magnetic resonance imaging (MRI) are increasingly able to detect fetal anomalies with potential syndromic implications. This case report details the prenatal diagnosis of Pallister-Hall syndrome, a rare autosomal dominant disorder, in a 31 year old primagravida referred for multidisciplinary evaluation at 28 weeks gestation for abnormal facies and abnormal hands and feet. Findings of bilateral polysyndactyly of the fetal hands, renal hypoplasia, a high arched palate, microphallus, probable imperforate anus and the classic finding of a hypothalamic hamartoma were all consistent with a clinical diagnosis of Pallister-Hall syndrome. Subsequent molecular studies confirmed the presence of a de novo GLI3 mutation. While prenatal diagnosis of Pallister-Hall has been reported previously based upon MRI diagnosis of a hypothalamic hamartoma, this case appears to be the first with prenatal molecular confirmation and provides yet another example of the importance and value of multifaceted prenatal 
assessment in the complete evaluation of fetal anomalies. Ultimately, this diagnosis allowed for both multidisciplinary genetic counseling in the prenatal period and specialized delivery planning with immediate multidisciplinary neonatal evaluation to optimize postnatal outcome.

\title{
What Is the Chance for Women with a History of Depression to Develop Psychosis in the Postpartum Period: Preliminary Data to Inform Prenatal Genetic Counseling Practice
}

\author{
C. Mighton ${ }^{1}$, A. Inglis ${ }^{1}$, C. Hippman ${ }^{1}$, P. Carrion ${ }^{1}$, W. Honer ${ }^{1}$, J. Austin ${ }^{1}$

\section{University of British Columbia}

Background: Postpartum psychosis (PPP) is the most serious of postpartum mental health problems, and constitutes a medical emergency. While women with a history of major depressive disorder (MDD) have higher chances for postpartum depressive and manic episodes, little is known about the chance for PPP. Further, though some research suggests that risk for PPP may be influenced by the sex of baby, data are inconsistent. Objectives: To determine the chance of PPP among women with a history of MDD, and to explore whether this is influenced by the sex of the baby. Methods: Primiparous women with a self-reported history of MDD were recruited, and the Structured Clinical Interview for DSM IV disorders was administered to confirm diagnosis and exclude women with any history of psychosis or bipolar disorder. The Positive and Negative Syndrome Scale (PANSS) was administered once during pregnancy and at 1 week, 1 month, and 3 months postpartum. Presence of psychosis was defined by a score above threshold in one or more of five key symptoms on the PANSS. To determine the impact of sex of baby on chance of PPP, we used Fisher's exact test. Results: Eleven of 60 participants (18.3\%) experienced PPP, which had postpartum onset for 6/11. Sex of the baby had no significant impact on the chance for PPP ( $p=$ 0.073). Conclusions: PPP occurs in the general population at a rate of $0.1 \%$, so at a rate of $18.3 \%$, seems to be considerably more common among women with MDD, but controlled studies involving diagnostic interviews are necessary to confirm this. However, these initial findings could help prenatal genetic counselors when discussing perinatal psychiatric risks with women with a history of MDD and suggest a rationale for monitoring women with a history of MDD for psychosis during the perinatal period.

\section{Making the Choice to Continue: How Noninvasive Prenatal Screening is Utilized in Southeastern United States}

\author{
J. Nichols ${ }^{1}$, M. Dudek ${ }^{2}$ \\ 1. Vanderbilt Medical Center \\ 2. Vanderbilt School of Medicine
}

Objective: To determine the number of patients who continue a pregnancy after receiving screen positive NIPS result. Background: Noninvasive prenatal screening (NIPS) is an option for prenatal risk assessment for chromosome aneuploidy in pregnancy. Current guidelines recommend that patients at high risk for chromosome abnormalities in their pregnancy (advanced maternal age, positive maternal serum screen, abnormal ultrasound, or family history) have pre-test counseling from a healthcare provider to obtain informed consent prior to electing NIPS. Disability advocates have voiced concern regarding the role of NIPS in the termination of pregnancies. Methods: REDCap, a web-based survey tool, was used to prospectively collect clinical information regarding pregnancy decisions from 737 high-risk patients electing NIPS from October 2010 through March 2014 in a southeastern academic medical center. All patients had informed consent by a boarded and licensed genetic counselor. Results: The screen positive rate in this data set was $3.7 \%$ with 27 screen positive results (21 Down syndrome; 6 trisomy 18). Ninety-two percent had ultrasound findings suspicious of chromosomal aneuploidy. Of the 26 with follow up data, screening results were confirmed with $35 \%$ (9) prenatally and $65 \%$ (17) postnataly. Majority of patients $(73 \%$ or $19 / 26)$ continued their pregnancy. Five (3 trisomy $21 ; 2$ trisomy 18$)$ pregnancies were electively terminated (19 \%). All patients returned to meet with the genetic counselor following a screen positive result except for one patient who moved out of state. Conclusions: NIPS provides a testing choice that was not previously available for the subset of at-risk patients who decline invasive testing. These patients were previously left with less accurate risk assessment provided by ultrasound alone. In this study NIPS provided reassurance to $96 \%$ of at-risk pregnancies. Those that had screen positive results choose to continue in $73 \%$ of cases.

\section{The Declining Rate of Amniocentesis Procedures at One Center}

\section{Rhee-Morris ${ }^{1}$, E. Love ${ }^{2}$}

\section{UC Davis Medical Center}

\section{UC Davis Health System}

Introduction: Is amniocentesis a procedure of the past at one northern California Prenatal Diagnosis Center? We sought to determine how the acceptance rate of the amniocentesis procedure had decreased over time. Methods: We queried our UC Davis Prenatal Diagnosis Center's database for the referral indication "advanced maternal age (AMA)" and "increased risk for trisomy 21 (T21) or trisomy 18 (T18)" seen between 1994 to 2013. A total of 12,511 women were seen for these indications at our main site and satellite sites. Results: The choice to have an amniocentesis has decreased over time, from 76 to $10 \%$ in our AMA population and 75 to $17 \%$ in our increased risk for T21 or T18 population. Conclusions: Amniocentesis procedures are becoming rarer but are still done at our center. Training Maternal-Fetal Medicine fellows to do amniocentesis procedures is increasingly difficult since they must do 50 amniocentesis (25 supervised) during their three fellowship years per the California Department of Public Health, Genetic Disease Screening Program guidelines noninvasive prenatal testing can screen for about $83 \%$ of chromosome abnormalities, but will miss approximately $17 \%$ of other chromosome aneuploidies. 
Challenges in Expanded Carrier Screening: Determining Accurate Detection Rates and Residual Carrier Risks

\author{
S. Rodriguez ${ }^{1}$, C. Rivera ${ }^{1}$, N. Kumar ${ }^{1}$, G. Kellogg ${ }^{1}$, A. Bisignano ${ }^{1}$
}

\title{
1. Recombine
}

Genetic counselors (GCs) commonly offer genetic carrier screening. To facilitate reproductive and medical decision-making based on carrier screening results, GCs estimate reproductive risk based on the disease carrier frequency and the detection rate of the test. Further, GCs also use detection rates as a way to compare the clinical utility of different genetic tests. In calculating detection rates for the 213 recessive genetic disorders included in our panel, we reviewed over 650 primary literature articles and found several recurrent inaccuracies and inconsistencies. Many of the studies we used to calculate detection rates had small sample sizes and/or were performed in isolated populations. However, this is inevitable given the rarity of genetic diseases. Additionally, several studies did not accurately report patient and genetic data. For instance, we found many studies where patients' racial/ethnic backgrounds were not clearly reported, thus affecting the accuracy of ethnicity-related residual carrier and reproductive risks. Furthermore, many studies excluded affected chromosomes with unknown or unidentifiable mutations from calculations, leading to overly high detection rates and falsely reduced residual carrier risks. As a result of these issues, we made efforts to report patient race/ethnicity in a clear and representative manner and calculate detection rates for diseases included in our panel based on original reports of the number of affected chromosomes carrying a specific mutation vs. total number of affected chromosomes studied. Given the importance of this information for genetic counselors in the preconception/prenatal setting, we aim to review these and other issues with detection rate calculations, outline the utility and limitations of detection rates, and propose methods for computing detection rates to ensure their clinical actionability. This is critical to ensure expanded carrier screening is performed in a clinically responsible manner.

Clinical Description of a Newborn Male with a Prenatal Diagnosis of 46,XY,del(16) (q12.1q21)dn

B. Tucker ${ }^{1}$, D. Hennerich ${ }^{2}$, S. Toomey ${ }^{2}$, R. Reynolds ${ }^{1}$, S. Reeves ${ }^{1}$

1. Colorado Fetal Care Center

2. Colorado Genetics Laboratory

Introduction: We present a case of a newborn male with a de novo interstitial deletion on chromosome 16. Case report: A 39-year-old G3P1011 was evaluated antenatally for a fetal diagnosis of growth restriction, congenital heart disease, and micrognathia. Amniocentesis revealed a de novo deletion on chromosome 16 initially characterized as 46,XY,rea(16) (q13q22). Subsequent fetal echocardiograms established a complete atrioventricular septal defect (AVSD), suspected tetrology of Fallot, mild hypoplasia of the right ventricle, bilateral superior vena cavas, small branch pulmonary arteries, and a prominent A wave reversal in the systemic veins. Fetal ultrasound was also significant for single umbilical artery, fetal growth restriction and placental insufficiency. Delivery occurred by repeat cesarean after rupture of membranes at $362 / 7$ weeks. The neonate was intubated for respiratory distress. Hypertelorism, downslanting palpebral fissures, small, low-set, and posteriorly rotated ears, marked retrognathia, and a cleft palate were noted. The left hand had a single palmar crease and the right had a bifid thumb. Toes were overlapping bilaterally. A complete, unbalanced AVSD and left superior vena cava were confirmed. The infant was treated for necrotizing enterocolitis on day 16. Due to subpulmonary stenosis, balloon valvuloplasty was performed at 22 days of life. A deep venous thrombosis developed and was treated with enoxaparin. He died at 27 days of life after developing altered mental status, dilated pupils, respiratory decompensation, acidosis and hyperkalemia. His final karyotype was 46,XY,del(16) (q12.1q21)dn. A chromosomal microarray revealed a 12.1 Mb deletion spanning linear positions 51,701,772 -63,686,371. Discussion: Approximately 30 interstitial deletions on chromosome 16q with clusters at 16q11q13 and 16q22 have been reported in the medical literature. This case presents the clinical phenotype and outcome of a neonate with a $12.1 \mathrm{Mb}$ deletion in this region, which may help in future counseling for patients with similar deletions.

Parent Perspectives on Support Received from Physicians and Genetic Counselors following a Decision to Continue a Pregnancy with a Prenatal Diagnosis of Trisomy 13/18

\author{
S. Wallace ${ }^{1}$, S. Gilvary ${ }^{1}$, S. Dolan ${ }^{2}$ \\ 1. Sarah Lawrence College \\ 2. March of Dimes
}

Background: Families that choose to continue a pregnancy with a prenatal diagnosis of trisomy 13/18 are a minority that present unique challenges for those in charge of their care. This study investigated the extent to which these patients felt supported by their healthcare providers, and any differences in the perceived level of support experienced by those working with a physician vs. those working with a genetic counselor. Methods: SOFT and Hope for Trisomy distributed an 84-question online survey to their members. Means, standard deviations and chi-squared analysis were used to evaluate responses. Results: One hundred ninety six surveys were returned and 114 included in the final analysis. Respondents were more likely to indicate that genetic counselors rather than physicians provided unbiased information in a way that they were able to understand. Forty-seven percent felt that their genetic counselor would support either a decision to continue or a decision to terminate, as compared to $37 \%$ for physicians. Review of qualitative responses found that the portrayal of trisomy $13 / 18$ by healthcare providers in terms of lethality, morbidity and burden of the condition could be perceived as directive. 13 out of 99 respondents felt their physician said nothing directive. Comparatively, over half felt that there was nothing the genetic counselor said that was portrayed as directive. Conclusion: A study of families who chose to continue a pregnancy after a diagnosis of trisomy 13 or 18 indicates that involving genetic counselors may be beneficial, especially in the area of information giving. Families report a lack of support from medical caregivers, which might be ameliorated by providing up-to-date written resources and referrals to support groups for people who have experienced a similar diagnosis. 
Potential Biological Explanations for no Results for Sex Chromosome Aneuploidy Assessment Using Directed Cell-Free DNA Analysis: A Summary of Three Cases

\author{
K. White', M. Lyons ${ }^{2}$, B. Peach ${ }^{3}$, M. Menezes ${ }^{4}$, R. Doyel ${ }^{1}$, A. Batey ${ }^{1}$ \\ 1. Ariosa Diagnostics \\ 2. Monmouth Medical Center \\ 3. $s o+g i$ \\ 4. Monash Ultrasound for Women
}

In the past few years, analysis of cell-free DNA (cfDNA) isolated from maternal plasma for fetal trisomy 21,18 , and 13 risk assessment has been introduced into prenatal care across the globe. This technology also makes it possible to screen for sex chromosome aneuploidy; however, regardless of the cfDNA test methodology used, small validation studies have demonstrated lower test performance than for autosomal trisomy. In the scientific literature, much of the focus has been on the predictive capabilities of cfDNA testing in successful analyses. Less attention has been given to analyses for which results cannot be provided. For sex chromosome aneuploidy assessment, there are instances where sex chromosome copy number cannot be determined and a statistical likelihood of a disomic (XX, XY) or non-disomic (X, XXX, XXY, XYY, and XXYY) genotype cannot be established. Such results, although infrequent, may be due to technical or biological factors and can represent a counseling challenge. We present three cases of testing using directed cfDNA analysis that resulted in trisomy risk assessment but no results for sex chromosome aneuploidy assessment. Further evaluation in each case identified a potential explanation. In case \#1, the patient opted to pursue diagnostic testing by amniocentesis, which revealed a 45,X[4]/ 46,XY[14] fetal karyotype. In case \#2, peripheral blood chromosome analysis identified a 45,X[8]/46,XX[54] maternal karyotype. In case \#3, a previously unreported twin gestation was revealed. Each case is presented as an example of a different biological factor that can contribute to no results being returned for risk of sex chromosome aneuploidy. Since extensive further evaluation is rarely indicated or pursued, the frequencies of these biological explanations are unknown. Qualitatively, these cases underscore the importance of inclusion of the possibility of no results into pre-test counseling and add to the base of knowledge for use in post-test counseling in these situations. 